\title{
A Generalized Sharp Whitney Theorem for Jets
}

\section{Charles Fefferman}

\begin{abstract}
Suppose that, for each point $x$ in a given subset $E \subset \mathbb{R}^{n}$, we are given an $m$-jet $f(x)$ and a convex, symmetric set $\sigma(x)$ of $m$-jets at $x$. We ask whether there exist a function $F \in C^{m, \omega}\left(\mathbb{R}^{n}\right)$ and a finite constant $M$, such that the $m$-jet of $F$ at $x$ belongs to $f(x)+M \sigma(x)$ for all $x \in E$. We give a necessary and sufficient condition for the existence of such $F, M$, provided each $\sigma(x)$ satisfies a condition that we call "Whitney $\omega$-convexity".
\end{abstract}

\section{Introduction}

Generalizing $[9,11]$, we obtain here a result used in [10] as a key step in solving the following problem.

Whitney's Extension Problem: Let $m \geq 1$, and let $\varphi: E \longrightarrow \mathbb{R}$, with $E \subseteq \mathbb{R}^{n}$ compact. How can we tell whether $\varphi$ extends to a $C^{m}$ function on $\mathbb{R}^{n}$ ?

We start by recalling the result of [11], and then discuss the main theorem of this paper. We next recall from [10] the solution of Whitney's extension problem. Our introduction ends with a brief historical discussion, touching on the work of Whitney [19, 20, 21], Glaeser [12], Brudnyi-Shvartsman $[3, \ldots, 7$ and $14,15,16]$, Zobin $[22,23]$, and Bierstone-Milman-Pawlucki $[1,2]$.

The result of [11] deals with $C^{m, \omega}\left(\mathbb{R}^{n}\right)$, the space of functions $F: \mathbb{R}^{n} \rightarrow \mathbb{R}$ whose derivatives through order $m$ are bounded and have modulus of continuity $\omega$. We assume that $\omega$ is a "regular modulus of continuity" as defined in Section 2 below. This is a very mild assumption. We seek a function $F \in C^{m, \omega}\left(\mathbb{R}^{n}\right)$ whose restriction to a given set $E$ agrees with a given function $f$ to a given tolerance $\sigma$. The main theorem of [11] is as follows.

2000 Mathematics Subject Classification: 49K24, 52A35.

Keywords: Extension problems, Whitney convexity, Whitney $\omega$-convexity. 
Theorem 1 Given $m, n \geq 1$, there exists $k^{\#}$, depending only on $m$ and $n$, for which the following holds.

Let $\omega$ be a regular modulus of continuity, let $E \subseteq \mathbb{R}^{n}$; and let $f: E \longrightarrow \mathbb{R}$ and $\sigma: E \longrightarrow[0, \infty)$ be given functions on $E$. Suppose that, given $S \subseteq E$ with cardinality at most $k^{\#}$, there exists $F^{S} \in C^{m, \omega}\left(\mathbb{R}^{n}\right)$, satisfying

$$
\left\|F^{S}\right\|_{C^{m, \omega}\left(\mathbb{R}^{n}\right)} \leq 1 \quad \text { and } \quad\left|F^{S}(x)-f(x)\right| \leq \sigma(x) \quad \text { for all } x \in S
$$

Then there exists $F \in C^{m, \omega}\left(\mathbb{R}^{n}\right)$, satisfying

$$
\|F\|_{C^{m, \omega}\left(\mathbb{R}^{n}\right)} \leq A, \quad \text { and } \quad|F(x)-f(x)| \leq A \sigma(x) \quad \text { for all } x \in E .
$$

Here, $A$ is a constant depending only on $m$ and $n$.

Thus, to decide whether there exist a function $F \in C^{m, \omega}\left(\mathbb{R}^{n}\right)$ and a finite constant $M$, such that $|F(x)-f(x)| \leq M \cdot \sigma(x)$ for all $x \in E$, it is enough to examine finite subsets $S \subseteq E$ with cardinality at most $k^{\#}$.

Our goal here is to prove a version of Theorem 1 in which the condition $|F(x)-f(x)| \leq \sigma(x)$ is replaced by the requirement that the $m$-jet of $F$ at $x$ belong to a prescribed convex set. We write $\mathcal{R}_{x}$ for the ring of $m$-jets of smooth, real-valued functions at $x \in \mathbb{R}^{n}$; and we write $J_{x}(F)$ for the $m$-jet of $F$ at $x$.

Now suppose that, for each point $x \in E$, we are given an $m$-jet $f(x) \in$ $\mathcal{R}_{x}$, and a closed, symmetric convex subset $\sigma(x) \subseteq \mathcal{R}_{x}$. Let $\omega$ be a regular modulus of continuity. We ask: How can we decide whether there exist $F \in C^{m, \omega}\left(\mathbb{R}^{n}\right)$ and a constant $A<\infty$ such that $J_{x}(F)-f(x) \in A \cdot \sigma(x)$ for all $x \in E$ ?

We want to prove an analogue of Theorem 1 for this problem. We will need some restriction on the set $\sigma(x)$, or else the desired analogue of Theorem 1 will be obviously false. (For instance, any linear PDE $L F=g$ has the form $J_{x}(F)-f(x) \in \sigma(x)$ for a suitable jet $f(x)$ and linear subspace $\left.\sigma(x) \subseteq \mathcal{R}_{x}.\right)$

Two natural questions come to mind:

- Which hypotheses on $\sigma(x)$ allow us to carry over the proof of Theorem 1 from [11] to our present setting?

- Which hypotheses on $\sigma(x)$ allow us to apply the analogue of Theorem 1 to solve Whitney's extension problem as in [10]?

Interestingly, these two questions have very similar answers.

The correct hypothesis on $\sigma(x)$ is "Whitney $\omega$-convexity". To define this notion, we introduce a bit more notation. 
We fix $m, n \geq 1$, and let $\mathcal{P}$ denote the vector space of all (real-valued) $m^{\text {th }}$ degree polynomials on $\mathbb{R}^{n}$. For functions $F$, we identify the $m$-jet $J_{x}(F)$ with the Taylor polynomial

$$
y \mapsto \sum_{|\alpha| \leq m} \frac{1}{\alpha !}\left(\partial^{\alpha} F(x)\right) \cdot(y-x)^{\alpha} .
$$

Thus, the ring $\mathcal{R}_{x}$ of $m$-jets at $x$ is identified with $\mathcal{P}$ as a vector space; and we regard elements of $\mathcal{R}_{x}$ as polynomials $P \in \mathcal{P}$.

We can now define the notion of "Whitney $\omega$-convexity".

Let $\omega$ be a regular modulus of continuity, let $\sigma \subseteq \mathcal{R}_{x_{0}}$ be a set of $m$-jets at $x_{0}$, and let $A$ be a positive number.

We say that $\sigma$ is "Whitney $\omega$-convex, with Whitney constant $A$ ", if the following conditions are satisfied:

- $\sigma$ is closed, convex, and symmetric (i.e., $P \in \sigma$ if and only if $-P \in \sigma$ ).

- Suppose $P \in \sigma, Q \in \mathcal{R}_{x_{0}}$, and $\delta \in(0,1]$. Assume that $P$ and $Q$ satisfy the estimates

$$
\left|\partial^{\beta} P\left(x_{0}\right)\right| \leq \omega(\delta) \cdot \delta^{m-|\beta|} \text { and }\left|\partial^{\beta} Q\left(x_{0}\right)\right| \leq \delta^{-|\beta|} \text { for }|\beta| \leq m .
$$

Then $P \cdot Q \in A \sigma$, where the dot denotes multiplication in $\mathcal{R}_{x_{0}}$.

If we omit the factor $\omega(\delta)$ in the above estimates, then we arrive at the closely related notion of "Whitney convexity". (See [10], and Section 2 below.) Note that, if $\sigma$ is Whitney convex, then $\sigma$ is also Whitney $\omega$-convex, for any regular modulus of continuity $\omega$. (This follows at once from the above definitions, since $\omega(\delta) \leq 1$ for $\delta \in(0,1]$ and $\omega$ a regular modulus of continuity; see Section 2.)

Our analogue of Theorem 1 for Whitney $\omega$-convex sets is as follows.

Theorem 2 Given $m, n \geq 1$, there exists $k^{\#}$, depending only on $m$ and $n$, for which the following holds.

Let $\omega$ be a regular modulus of continuity, let $E \subseteq \mathbb{R}^{n}$, and let $A>0$. For each $x \in E$, suppose we are given an $m$-jet $f(x) \in \mathcal{R}_{x}$, and a Whitney $\omega$-convex subset $\sigma(x) \subseteq \mathcal{R}_{x}$ with Whitney constant $A$. Suppose that, given $S \subseteq E$ with cardinality at most $k^{\#}$, there exists $F^{S} \in C^{m, \omega}\left(\mathbb{R}^{n}\right)$, satisfying

$$
\left\|F^{S}\right\|_{C^{m, \omega}\left(\mathbb{R}^{n}\right)} \leq 1, \quad \text { and } \quad J_{x}\left(F^{S}\right)-f(x) \in \sigma(x) \quad \text { for all } x \in S .
$$

Then there exists $F \in C^{m, \omega}\left(\mathbb{R}^{n}\right)$, satisfying

$$
\|F\|_{C^{m, \omega}\left(\mathbb{R}^{n}\right)} \leq A^{\prime}, \quad \text { and } \quad J_{x}(F)-f(x) \in A^{\prime} \cdot \sigma(x) \quad \text { for all } x \in E .
$$

Here, $A^{\prime}$ depends only on $m, n$, and on the Whitney constant $A$. 
The purpose of this paper is to prove Theorem 2, by carrying over the proof of Theorem 1 from [11]. We make a few remarks about Theorem 2, and about the notions of Whitney $\omega$-convexity and Whitney convexity.

First of all, note that Theorem 2 immediately implies Theorem 1. (In fact, given a function $\sigma: E \longrightarrow[0, \infty)$, we define a set $\hat{\sigma}\left(x_{0}\right)$ of $m$-jets, for each $x_{0} \in E$, by setting

$$
\hat{\sigma}\left(x_{0}\right)=\left\{P \in \mathcal{P}:\left|P\left(x_{0}\right)\right| \leq \sigma\left(x_{0}\right)\right\} .
$$

One checks trivially that $\hat{\sigma}\left(x_{0}\right)$ is Whitney convex with Whitney constant 1 , and that Theorem 2 for $\hat{\sigma}$ is equivalent to Theorem 1 for $\sigma$.) Since the proof of Theorem 2 is close to that of Theorem 1, and is presented here in detail, we will not be publishing [11].

Next, note that Theorem 2 yields the following corollary.

Theorem 3 Let $m, n \geq 1$. Then there exists a constant $k^{\#}$, depending only on $m$ and $n$, for which the following holds:

Let $\omega$ be a regular modulus of continuity, and let $E \subset \mathbb{R}^{n}$ be an arbitrary subset. Suppose that for each $x \in E$ we are given an $m$-jet $f(x) \in \mathcal{R}_{x}$ and subset $\sigma(x) \subset \mathcal{R}_{x}$.

Assume that each $\sigma(x)$ is Whitney convex, with a Whitney constant $A_{0}$ independent of $x$.

Assume also that, given any subset $S \subset E$ with cardinality at most $k^{\#}$, there exists a map $x \mapsto P^{x}$ from $S$ into $\mathcal{P}$, with

(a) $P^{x} \in f(x)+\sigma(x)$ for all $x \in S$;

(b) $\left|\partial^{\beta} P^{x}(x)\right| \leq 1$ for all $x \in S,|\beta| \leq m$; and

(c) $\left|\partial^{\beta}\left(P^{x}-P^{y}\right)(y)\right| \leq \omega(|x-y|) \cdot|x-y|^{m-|\beta|}$ for $|\beta| \leq m,|x-y| \leq 1$, $x, y \in S$.

Then there exists $F \in C^{m, \omega}\left(\mathbb{R}^{n}\right)$, with $\|F\|_{C^{m, \omega}\left(\mathbb{R}^{n}\right)} \leq A_{1}$, and

$$
J_{x}(F) \in f(x)+A_{1} \sigma(x) \quad \text { for all } x \in E .
$$

Here, $A_{1}$ depends only on $m, n$ and the Whitney constant $A_{0}$.

To deduce Theorem 3 from Theorem 2, we simply recall that Whitney convexity implies Whitney $\omega$-convexity, and we invoke Lemma 2.1 from Section 2 below.

Theorem 3 is a crucial step in our paper [10] solving Whitney's extension problem for $C^{m}$. 
The notions of Whitney convexity and Whitney $\omega$-convexity are somewhat mysterious. On the one hand, there are interesting examples of Whitney convex sets.

For instance, let $E \subset \mathbb{R}^{n}$ be given, and let $x_{0}$ be a point of $\mathbb{R}^{n}$, possibly in or close to $E$. Then the closure of the set

$$
\hat{\sigma}\left(x_{0}\right)=\left\{J_{x_{0}}(F):\|F\|_{C^{m}\left(\mathbb{R}^{n}\right)} \leq 1 \text { and } F=0 \text { on } E\right\} \subseteq \mathcal{R}_{x_{0}}
$$

is easily seen to be Whitney convex, with a Whitney constant depending only on $m$ and $n$. (See the proof of Lemma 5.3 in [10].)

On the other hand, I don't know how to decide efficiently whether a given set $\sigma \subseteq \mathcal{R}_{x_{0}}$ is Whitney convex, or Whitney $\omega$-convex; or how to compute the order of magnitude of the best Whitney constant for $\sigma$.

It would be interesting to understand these issues.

Next, we recall our solution of Whitney's extension problem from [10].

Let $\varphi: E \longrightarrow \mathbb{R}$ be given, with $E \subset \mathbb{R}^{n}$ compact, as in Whitney's problem. By induction on $\ell \geq 0$, we define an affine subspace $H_{\ell}\left(x_{0}\right) \subseteq \mathcal{P}$ for each point $x_{0} \in E$. We start with

$$
H_{0}\left(x_{0}\right)=\left\{P \in \mathcal{P}: P\left(x_{0}\right)=\varphi\left(x_{0}\right)\right\} \quad \text { for } x_{0} \in E .
$$

The induction step is as follows. Fix $\ell \geq 0$, and suppose we have defined $H_{\ell}(x)$ for all $x \in E$. We will define an affine subspace $H_{\ell+1}\left(x_{0}\right) \subseteq H_{\ell}\left(x_{0}\right)$ for each $x_{0} \in E$. To do so, let $\bar{k}$ be a large enough constant, depending only on $m$ and $n$. Let $B(x, r)$ denote the open ball of radius $r$ about $x$ in $\mathbb{R}^{n}$. We say that a given $P_{0} \in H_{\ell}\left(x_{0}\right)$ belongs to $H_{\ell+1}\left(x_{0}\right)$ if the following condition holds:

Given $\epsilon>0$ there exists $\delta>0$ such that, for any $x_{1}, \ldots, x_{\bar{k}} \in E \cap B\left(x_{0}, \delta\right)$, there exist $P_{1} \in H_{\ell}\left(x_{1}\right), \ldots, P_{\bar{k}} \in H_{\ell}\left(x_{\bar{k}}\right)$, with

$$
\left|\partial^{\alpha}\left(P_{i}-P_{j}\right)\left(x_{j}\right)\right| \leq \epsilon\left|x_{i}-x_{j}\right|^{m-|\alpha|} \text { for }|\alpha| \leq m, 0 \leq i, j \leq \bar{k} .
$$

Note that $H_{\ell+1}\left(x_{0}\right)$ may be empty. By convention, we allow the empty set as an affine subspace of $\mathcal{P}$.

In principle, the $H_{\ell}\left(x_{0}\right)$ are computable from $\varphi: E \longrightarrow \mathbb{R}$.

The significance of the subspaces $H_{\ell}\left(x_{0}\right)$ is that, whenever $F \in C^{m}\left(\mathbb{R}^{n}\right)$ with $F=\varphi$ on $E$, then $J_{x_{0}}(F) \in H_{\ell}\left(x_{0}\right)$ for any $\ell \geq 0$ and $x_{0} \in E$. (This follows from an easy induction on $\ell$ using Taylor's theorem.) In particular, if any $H_{\ell}\left(x_{0}\right)$ is empty, then obviously $\varphi$ cannot admit a $C^{m}$ extension $F$. Conversely, [10] uses Theorem 3 to demonstrate the following result. 
Theorem 4 Let $\ell=2 \cdot \operatorname{dim} \mathcal{P}+1$.

(A) If $H_{\ell}\left(x_{0}\right)$ is non-empty for every $x_{0} \in E$, then $\varphi$ extends to a $C^{m}$ function $F$ on $\mathbb{R}^{n}$.

(B) Suppose $\varphi$ extends to a $C^{m}$ function on $\mathbb{R}^{n}$. Let $x_{0} \in E$. Then, given $P_{0} \in H_{\ell}\left(x_{0}\right)$, there exists $F \in C^{m}\left(\mathbb{R}^{n}\right)$ with $F=\varphi$ on $E$ and $J_{x_{0}}(F)=P_{0}$.

Theorem 4 solves Whitney's problem, and also computes the space of all possible $m$-jets at a given $x_{0} \in E$ of functions $F \in C^{m}\left(\mathbb{R}^{n}\right)$ with $F=\varphi$ on $E$. See Bierstone-Milman-Pawlucki [1,2]. Our proof of Theorem 4 in [10] uses Theorem 3 from this paper, which is called the "Generalized Sharp Whitney Theorem" in [10].

We give a brief historical discussion of Whitney's extension problem. Whitney began the subject in $[19,20,21]$ in 1934 , by settling the extension problem for the case of $C^{m}\left(\mathbb{R}^{1}\right)$, and by proving the classical Whitney extension theorem.

In 1958, G. Glaeser [12] solved Whitney's problem for $C^{1}\left(\mathbb{R}^{n}\right)$ by introducing a geometrical object called the "iterated paratangent space". Glaeser's work influenced all later work on the subject. A series of papers by Y. Brudnyi and P. Shvartsman $[3, \ldots, 7$ and $14,15,16]$ studied the analogue of Whitney's problem for $C^{m, \omega}\left(\mathbb{R}^{n}\right)$ and other function spaces. Among their conjectures is the case $\sigma \equiv 0$ of Theorem 1 . Among their results is the case $\sigma \equiv 0$, $m=1$ of Theorem 1 , with the sharp constant $k^{\#}=3 \cdot 2^{n-1}$, proven by the elegant method of "Lipschitz selection", which has independent interest. We refer the reader to $[3, \ldots, 7$ and $14,15,16]$ for these and other related results and conjectures. See also N. Zobin [22,23], for the solution of a problem that may prove to be closely related to the ones discussed here.

The next progress on Whitney's problem was the work of BierstoneMilman-Pawlucki [1]. They introduced an analogue of Glaeser's iterated paratangent space relevant to $C^{m}\left(\mathbb{R}^{n}\right)$. The conjectured a complete solution of Whitney's extension problem based on their paratangent space, and they found supporting evidence for their conjecture. (A version of their conjecture holds for sub-analytic sets $E$.) Theorem 4 is equivalent by duality to the Bierstone-Milman-Pawlucki conjectures [1] with their paratangent space replaced by a natural variant. (See [2].) Some modification of the condition in [1] is required to treat general compact sets without loss of derivatives; see a forthcoming paper by Bierstone, Milman, Pawlucki and the author.

It is a pleasure to thank E. Bierstone and P. Milman for very useful conversations and to acknowledge the influence of [1], as well as to thank the Courant Institute of Mathematical Sciences where this work was carried out. I am particularly grateful to Gerree Pecht for making special efforts to $\mathrm{TEX}_{\mathrm{E}}$ this paper quickly and accurately. We now begin the work of proving Theorem 2. 


\section{Notation and Preliminaries}

A "regular modulus of continuity" is a function $\omega(t)$, defined for $0 \leq t \leq 1$, and satisfying the following conditions:

(1) $\omega(0)=\lim _{t \rightarrow 0+} \omega(t)=0$, and $\omega(1)=1$.

(2) $\omega(t)$ is increasing (not necessarily strictly) on $[0,1]$.

(3) $\omega(t) / t$ is decreasing (not necessarily strictly) on $(0,1]$.

Note the obvious estimates:

$$
\begin{array}{ll}
\omega(t) \geq t & \text { for } t \in[0,1] \\
\omega(t) \leq \omega\left(C_{1} t\right) \leq C_{1} \omega(t) & \text { for } C_{1} \geq 1, C_{1} t \leq 1 ; \text { and } \\
\omega(t) \geq \omega\left(c_{1} t\right) \geq c_{1} \omega(t) & \text { for } 0<c_{1} \leq 1, t \in[0,1] .
\end{array}
$$

These estimates are immediate from (1), (2), (3).

Suppose $\omega$ is a regular modulus of continuity, and suppose $m \geq 0$. We define $C^{m, \omega}\left(\mathbb{R}^{n}\right)$ as the space of all $C^{m}$ functions $F: \mathbb{R}^{n} \rightarrow \mathbb{R}$ for which the norm

$$
\|F\|_{C^{m, \omega}\left(\mathbb{R}^{n}\right)}=\max \left\{\max _{|\beta| \leq m} \sup _{x \in \mathbb{R}^{n}}\left|\partial^{\beta} F(x)\right|, \max _{|\beta|=m} \sup _{\substack{x, y \in \mathbb{R}^{n} \\ 0<|x-y| \leq 1}} \frac{\left|\partial^{\beta} F(x)-\partial^{\beta} F(y)\right|}{\omega(|x-y|)}\right\}
$$

is finite.

Note that we get an equivalent norm by allowing all $\beta$ with $|\beta| \leq m$ in the second sup.

We also define $C_{\ell o c}^{m, \omega}\left(\mathbb{R}^{n}\right)$ as the space of all functions $F$ that agree with some $F_{K} \in C^{m, \omega}\left(\mathbb{R}^{n}\right)$ on any given compact set $K \subset \mathbb{R}^{n}$. As usual, $C_{\text {loc }}^{m}\left(\mathbb{R}^{n}\right)$ denotes the space of functions $F$ with $m$ continuous derivatives, without any global boundedness assumption on $F$ or its derivatives.

We apply repeatedly the following obvious consequence of Taylor's Theorem: Let $\omega$ be a regular modulus continuity.

Suppose $F \in C_{\ell o c}^{m}\left(\mathbb{R}^{n}\right)$, with

$$
\left|\partial^{\beta} F(x)-\partial^{\beta} F(y)\right| \leq M \cdot \omega(|x-y|)
$$

for $|\beta|=m, x, y \in \mathbb{R}^{n},|x-y| \leq 1$. Then for $|\beta| \leq m,|x-y| \leq 1$, we have

$$
\left|\partial^{\beta} F(y)-\sum_{|\gamma| \leq m-|\beta|} \frac{1}{\gamma !}\left(\partial^{\beta+\gamma} F(x)\right) \cdot(y-x)^{\gamma}\right| \leq C M|x-y|^{m-|\beta|} \omega(|x-y|)
$$

with $C$ depending only on $m$ and $n$. 
In particular,

$$
\begin{aligned}
\mid \partial^{\beta} F(y)-\sum_{|\gamma| \leq m-|\beta|} \frac{1}{\gamma !} & \left(\partial^{\beta+\gamma} F(x)\right) \cdot(y-x)^{\gamma} \mid \\
& \leq C\|F\|_{C^{m, \omega}\left(\mathbb{R}^{n}\right)}|x-y|^{m-|\beta|} \omega(|x-y|) .
\end{aligned}
$$

for $|x-y| \leq 1,|\beta| \leq m$.

We fix $m, n \geq 1$ throughout this paper. We recall the following from the Introduction.

We let $\mathcal{P}$ denote the vector space of all real-valued polynomials of degree $\leq m$ on $\mathbb{R}^{n}$ and we let $D=\operatorname{dim} \mathcal{P}$.

If $F \in C_{\ell o c}^{m}\left(\mathbb{R}^{n}\right)$ and $y \in \mathbb{R}^{n}$, then we write $J_{y}(F)$ for the $m$-jet of $F$ at $y$, i.e., the polynomial

$$
x \mapsto \sum_{|\beta| \leq m} \frac{1}{\beta !}\left(\partial^{\beta} F(y)\right) \cdot(x-y)^{\beta} .
$$

We write $\mathcal{R}_{y}$ for the ring of jets at $y$. More precisely, $\mathcal{R}_{y}=\mathcal{P}$, with the multiplication operator that gives

$$
P \cdot Q=S \quad(P, Q, S \in \mathcal{P}) \quad \text { if and only if } \quad \partial^{\beta}(P Q-S)(y)=0
$$

for $|\beta| \leq m$, where $P Q$ denotes the ordinary product of polynomials.

Fix $y \in \mathbb{R}^{n}, A>0$, and let $\Omega$ be a subset of $\mathcal{R}_{y}$. Then, as in the Introduction, we say that $\Omega$ is "Whitney convex at $y$ with Whitney constant $A$ " if the following conditions are satisfied.

(a) $\Omega$ is closed, convex, and symmetric about the origin. (That is, $P \in \Omega$ if and only if $-P \in \Omega$.)

(b) Let $P \in \Omega, Q \in \mathcal{P}, 0<\delta \leq 1$ be given. Assume that

$$
\left|\partial^{\alpha} P(y)\right| \leq \delta^{m-|\alpha|} \quad \text { and } \quad\left|\partial^{\alpha} Q(y)\right| \leq \delta^{-|\alpha|}, \quad \text { for }|\alpha| \leq m .
$$

Let $Q \cdot P$ denote the product of $Q$ and $P$ in $\mathcal{R}_{y}$. Then $Q \cdot P$ belongs to $A \cdot \Omega$.

Note that if instead we have

$$
\left|\partial^{\alpha} P(y)\right| \leq M_{1} \delta^{m-|\alpha|} \quad \text { and } \quad\left|\partial^{\alpha} Q(y)\right| \leq M_{2} \delta^{-|\alpha|}
$$

for $|\alpha| \leq m$, with $M_{1} \geq 1$, then we obtain $Q \cdot P \in A M_{1} M_{2} \Omega$. 
Similarly, suppose $y \in \mathbb{R}^{n}, A>0, \Omega \subseteq \mathcal{R}_{y}$, and let $\omega$ be a regular modulus of continuity. Then we say that $\Omega$ is "Whitney $\omega$-convex at $y$ with Whitney constant $A$ " if the following conditions are satisfied.

(a) $\Omega$ is closed, convex, and symmetric about the origin.

(b) Let $P \in \Omega, Q \in \mathcal{P}, 0<\delta \leq 1$ be given. Assume that

$$
\left|\partial^{\beta} P(y)\right| \leq \omega(\delta) \cdot \delta^{m-|\beta|} \text { and }\left|\partial^{\beta} Q(y)\right| \leq \delta^{-|\beta|}, \quad \text { for }|\beta| \leq m .
$$

Let $Q \cdot P$ denote the product of $Q$ and $P$ in $\mathcal{R}_{y}$. Then $Q \cdot P$ belongs to $A \cdot \Omega$.

Note that if $\sigma \subseteq \mathcal{R}_{x}$ is Whitney convex, (with Whitney constant $A$ ), then it is Whitney $\omega$-convex for any regular modulus of continuity, again with Whitney constant $A$.

If $\beta, \alpha$ are multi-indices, then $\delta_{\beta \alpha}$ denotes the Kronecker delta, equal to 1 if $\beta=\alpha$, and equal to zero otherwise.

We let $\mathcal{M}$ denote the set of multi-indices $\beta=\left(\beta_{1}, \ldots, \beta_{n}\right)$ of order $|\beta|=$ $\beta_{1}+\cdots+\beta_{n} \leq m$.

We write $\mathcal{M}^{+}$for the set of all multi-indices of order $\leq m+1$.

A subset $\mathcal{A} \subseteq \mathcal{M}$ is called "monotonic" if, for any $\alpha \in \mathcal{A}$ and $\gamma \in \mathcal{M}$, $\alpha+\gamma \in \mathcal{M}$ implies $\alpha+\gamma \in \mathcal{A}$.

We write $B(x, r)$ for the open ball of radius $r$, centered at $x \in \mathbb{R}^{n}$.

A cube $Q$ is defined as a Cartesian product $\left[a_{1}, b_{1}\right) \times \cdots \times\left[a_{n}, b_{n}\right) \subset \mathbb{R}^{n}$, with $b_{1}-a_{1}=b_{2}-a_{2}=\cdots=b_{n}-a_{n}$. The diameter of a cube $Q$ is denoted by $\delta_{Q}$. If $Q$ is a cube, then $Q^{*}$ denotes the cube concentric with $Q$, and having diameter $3 \delta_{Q}$. To "bisect" a cube is to subdivide it into $2^{n}$ congruent sub-cubes in the obvious way. Later on (in Section 11), we will fix a cube $Q^{\circ} \subset \mathbb{R}^{n}$. Once $Q^{\circ}$ is fixed, the collection of "dyadic" cubes consists of $Q^{\circ}$, together with all the cubes arising from $Q^{\circ}$ by bisecting $k$ times, for any $k \geq 1$. Note that, by this definition, every dyadic cube is contained in $Q^{\circ}$. Moreover, any dyadic cube $Q$ other than $Q^{\circ}$ arises by bisecting a "dyadic parent" $Q^{+}$, with $\delta_{Q^{+}}=2 \delta_{Q}$.

We will often be dealing with functions of $x \in \mathbb{R}^{n}$, parametrized by $y \in \mathbb{R}^{n}$. We denote these by $\varphi^{y}(x)$, or by $P^{y}(x)$ if $x \mapsto P^{y}(x)$ is a polynomial for each fixed $y$. When we write $\partial^{\beta} P^{y}(y)$, we mean $\left(\frac{\partial}{\partial x}\right)^{\beta} P^{y}(x)$ evaluated at $x=y$. We never use $\partial^{\beta} P^{y}(y)$ to denote the derivative of order $\beta$ of the function $y \mapsto P^{y}(y)$.

If $S$ is any finite set, then we write \# $(S)$ for the number of elements of $S$. For $S$ infinite, we define $\#(S)=\infty$. 
We close this section with the following result.

Lemma 2.1 Let $\omega$ be a regular modulus of continuity, and let $S \subset \mathbb{R}^{n}$ be a finite set. Suppose we are given an $m$-jet $P^{x} \in \mathcal{P}$ associated to each point $x \in S$. Assume that

(a) $\left|\partial^{\beta} P^{x}(x)\right| \leq 1$ for $|\beta| \leq m, x \in S$; and that

(b) $\left|\partial^{\beta}\left(P^{x}-P^{y}\right)(y)\right| \leq \omega(|x-y|) \cdot|x-y|^{m-|\beta|}$ for $|\beta| \leq m,|x-y| \leq 1$, $x, y \in S$.

Then there exists $F^{S} \in C^{m, \omega}\left(\mathbb{R}^{n}\right)$, with

$$
J_{x}\left(F^{S}\right)=P^{x} \quad \text { for all } x \in S \text {, and with }\left\|F^{S}\right\|_{C^{m, \omega}\left(\mathbb{R}^{n}\right)} \leq C .
$$

Here, $C$ depends only on $m$ and $n$.

This result follows from the usual proof of the standard Whitney extension theorem. (See $[13,17]$.)

Using Lemma 2.1, one sees that our present Theorem 2 trivially implies the "Generalized Sharp Whitney Theorem" stated in [10] i.e., our present Theorem 3.

\section{Order Relations on Multi-Indices}

We introduce order relations on multi-indices, and on subsets of $\mathcal{M}$ as in [9]. Let us recall these relations.

Suppose $\alpha=\left(\alpha_{1}, \ldots, \alpha_{n}\right)$ and $\beta=\left(\beta_{1}, \ldots, \beta_{n}\right)$ are distinct multi-indices.

Then we must have $\alpha_{1}+\cdots+\alpha_{k} \neq \beta_{1}+\cdots+\beta_{k}$ for some $k$. Let $\bar{k}$ denote the largest such $k$. Then we say that $\alpha<\beta$ if and only if $\alpha_{1}+\cdots+\alpha_{\bar{k}}<$ $\beta_{1}+\cdots+\beta_{\bar{k}}$. One checks easily that this defines an order relation. Next, suppose $\mathcal{A}$ and $\mathcal{B}$ are distinct subsets of $\mathcal{M}$. Then the symmetric difference $\mathcal{A} \Delta \mathcal{B}=(\mathcal{A} \backslash \mathcal{B}) \cup(\mathcal{B} \backslash \mathcal{A})$ is non-empty. Let $\alpha$ denote the least element of $\mathcal{A} \Delta \mathcal{B}$, under the above ordering on multi-indices. Then we say that $\mathcal{A}<\mathcal{B}$ if and only if $\alpha$ belongs to $\mathcal{A}$. Again, one checks easily that this defines an order relation. As in [9], we have the following elementary results.

Lemma 3.1 If $\alpha, \beta$ are multi-indices, and if $|\alpha|<|\beta|$, then $\alpha<\beta$.

Lemma 3.2 If $\mathcal{A} \subseteq \overline{\mathcal{A}} \subseteq \mathcal{M}$, then $\overline{\mathcal{A}} \leq \mathcal{A}$.

Lemma 3.3 Let $\mathcal{A} \subset \mathcal{M}$, and let $\phi: \mathcal{A} \rightarrow \mathcal{M}$. Suppose that

(1) $\phi(\alpha) \leq \alpha$ for all $\alpha \in \mathcal{A}$, and

(2) for each $\alpha \in \mathcal{A}$, either $\phi(\alpha)=\alpha$ or $\phi(\alpha) \notin \mathcal{A}$.

Then $\phi(\mathcal{A}) \leq \mathcal{A}$, with equality if and only if $\phi$ is the identity map. 


\section{Statement of Two Main Lemmas}

Fix $\mathcal{A} \subseteq \mathcal{M}$. We state two results involving $\mathcal{A}$.

Weak Main Lemma for $\mathcal{A}$ : There exists $k^{\#}$, depending only on $m$ and $n$, for which the following holds.

Suppose we are given constants $C, a_{0}$; a regular modulus of continuity $\omega$; a finite set $E \subset \mathbb{R}^{n}$; a point $y^{0} \in \mathbb{R}^{n}$; and a family of polynomials $P_{\alpha} \in \mathcal{P}$, indexed by $\alpha \in \mathcal{A}$. Suppose also that for each $x \in E$, we are given an $m$-jet $f(x) \in \mathcal{R}_{x}$ and a subset $\sigma(x) \subset \mathcal{R}_{x}$.

Assume that the following conditions are satisfied.

(WL0) For each $x \in E$, the set $\sigma(x)$ is Whitney $\omega$-convex at $x$ with Whitney constant $C$.

(WL1) $\partial^{\beta} P_{\alpha}\left(y^{0}\right)=\delta_{\beta \alpha}$ for all $\beta, \alpha \in \mathcal{A}$.

(WL2) $\left|\partial^{\beta} P_{\alpha}\left(y^{0}\right)-\delta_{\beta \alpha}\right| \leq a_{0}$ for all $\alpha \in \mathcal{A}, \beta \in \mathcal{M}$.

(WL3) Given $\alpha \in \mathcal{A}$ and $S \subset E$ with $\#(S) \leq k^{\#}$, there exists $\varphi_{\alpha}^{S} \in$ $C_{\ell o c}^{m, \omega}\left(\mathbb{R}^{n}\right)$, with

(a) $\left|\partial^{\beta} \varphi_{\alpha}^{S}(x)-\partial^{\beta} \varphi_{\alpha}^{S}(y)\right| \leq a_{0} \cdot \omega(|x-y|)$ for $|\beta|=m, x, y \in \mathbb{R}^{n}$, $|x-y| \leq 1$

(b) $J_{x}\left(\varphi_{\alpha}^{S}\right) \in C \sigma(x)$ for all $x \in S$; and

(c) $J_{y^{0}}\left(\varphi_{\alpha}^{S}\right)=P_{\alpha}$.

(WL4) Given $S \subset E$ with $\#(S) \leq k^{\#}$, there exists $F^{S} \in C^{m, \omega}\left(\mathbb{R}^{n}\right)$, with

(a) $\|F\|_{C^{m, \omega}\left(\mathbb{R}^{n}\right)} \leq C$; and

(b) $J_{x}\left(F^{S}\right) \in f(x)+C \sigma(x)$ for all $x \in S$.

(WL5) $a_{0}$ is less than a small enough positive constant determined by $C, m, n$.

Then there exists $F \in C^{m, \omega}\left(\mathbb{R}^{n}\right)$, with

(WL6) $\|F\|_{C^{m, \omega}\left(\mathbb{R}^{n}\right)} \leq C^{\prime}$, and

(WL7) $J_{x}(F) \in f(x)+C^{\prime} \sigma(x)$ for all $x \in E \cap B\left(y^{0}, c^{\prime}\right)$.

Here, $C^{\prime}$ and $c^{\prime}$ in (WL6,7) depend only on $C, m, n$. 
Strong Main Lemma for $\mathcal{A}$ : There exists $k^{\#}$, depending only on $m$ and $n$, for which the following holds.

Suppose we are given constants $C, \bar{a}_{0}$; a regular modulus of continuity $\omega$; a finite set $E \subset \mathbb{R}^{n}$; a point $y^{0} \in \mathbb{R}^{n}$; and a family of polynomials $P_{\alpha} \in \mathcal{P}$, indexed by $\alpha \in \mathcal{A}$.

Suppose also that, for each $x \in E$, we are given an $m$-jet $f(x) \in \mathcal{R}_{x}$ and a subset $\sigma(x) \subset \mathcal{R}_{x}$.

Assume that the following conditions are satisfied.

(SL0) For each $x \in E$, the set $\sigma(x)$ is Whitney $\omega$-convex at $x$, with Whitney constant $C$.

(SL1) $\partial^{\beta} P_{\alpha}\left(y^{0}\right)=\delta_{\beta \alpha}$ for all $\beta, \alpha \in \mathcal{A}$.

(SL2) $\left|\partial^{\beta} P_{\alpha}\left(y^{0}\right)\right| \leq C$ for all $\beta \in \mathcal{M}, \alpha \in \mathcal{A}$ with $\beta \geq \alpha$.

(SL3) Given $\alpha \in \mathcal{A}$ and $S \subset E$ with $\#(S) \leq k^{\#}$, there exists $\varphi_{\alpha}^{S} \in C_{\text {loc }}^{m, \omega}\left(\mathbb{R}^{n}\right)$, with

(a) $\left|\partial^{\beta} \varphi_{\alpha}^{S}(x)-\partial^{\beta} \varphi_{\alpha}^{S}(y)\right| \leq \bar{a}_{0} \omega(|x-y|)+C|x-y|$ for $|\beta|=m$, $x, y \in \mathbb{R}^{n},|x-y| \leq 1$;

(b) $J_{x}\left(\varphi_{\alpha}^{S}\right) \in C \sigma(x)$ for all $x \in S$; and

(c) $J_{y^{0}}\left(\varphi_{\alpha}^{S}\right)=P_{\alpha}$.

(SL4) Given $S \subset E$ with $\#(S) \leq k^{\#}$, there exists $F^{S} \in C^{m, \omega}\left(\mathbb{R}^{n}\right)$, with

(a) $\left\|F^{S}\right\|_{C^{m, \omega}\left(\mathbb{R}^{n}\right)} \leq C$, and

(b) $J_{x}\left(F^{S}\right) \in f(x)+C \sigma(x)$ for all $x \in S$.

(SL5) $\bar{a}_{0}$ is less than a small enough positive constant determined by $C, m, n$.

Then there exists $F \in C^{m, \omega}\left(\mathbb{R}^{n}\right)$, with

(SL6) $\|F\|_{C^{m, \omega}\left(\mathbb{R}^{n}\right)} \leq C^{\prime}$, and

(SL7) $J_{x}(F) \in f(x)+C^{\prime} \sigma(x)$ for all $x \in E \cap B\left(y^{0}, c^{\prime}\right)$.

Here, $C^{\prime}$ and $c^{\prime}$ in $(S L 6,7)$ depend only on $C, m, n$. 


\section{Plan of the Proof}

We will establish the following results.

Lemma 5.1 The Weak Main Lemma and the Strong Main Lemma both hold for $\mathcal{A}=\mathcal{M}$.

(Note that $\mathcal{A}=\mathcal{M}$ is minimal for the order relation $<$.)

Lemma 5.2 Fix $\mathcal{A} \subset \mathcal{M}$ with $\mathcal{A} \neq \mathcal{M}$. Assume that the Strong Main Lemma holds for each $\overline{\mathcal{A}}<\mathcal{A}$. Then the Weak Main Lemma holds for $\mathcal{A}$.

Lemma 5.3 Fix $\mathcal{A} \subset \mathcal{M}$, and assume that the Weak Main Lemma holds for each $\overline{\mathcal{A}} \leq \mathcal{A}$. Then the Strong Main Lemma holds for $\mathcal{A}$.

Once we establish these lemmas, the two Main Lemmas must hold for all $\mathcal{A} \subseteq \mathcal{M}$, by induction on $\mathcal{A}$. In particular, taking $\mathcal{A}$ to be the empty set in, say, the Weak Main Lemma, we see that hypotheses (WL1,2,3) hold vacuously, and that the constant $a_{0}$ appears only in hypothesis (WL5). Hence, we obtain the following result.

Local Theorem: There exists $k^{\#}$, depending only on $m$ and $n$, for which the following holds.

Suppose we are given a regular modulus of continuity $\omega$; a finite set $E \subset \mathbb{R}^{n}$; and, for each $x \in E$, an $m$-jet $f(x) \in \mathcal{R}_{x}$ and a subset $\sigma(x) \subset \mathcal{R}_{x}$.

Assume that the following conditions are satisfied.

(I) For each $x \in E$, the set $\sigma(x)$ is Whitney $\omega$-convex at $x$, with Whitney constant $C$.

(II) Given $S \subset E$ with $\#(S) \leq k^{\#}$, there exists $F^{S} \in C^{m, \omega}\left(\mathbb{R}^{n}\right)$, with $\left\|F^{S}\right\|_{C^{m, \omega}\left(\mathbb{R}^{n}\right)} \leq C$, and $J_{x}(F) \in f(x)+C \cdot \sigma(x)$ for each $x \in S$.

Let $y^{0} \in \mathbb{R}^{n}$ be given. Then there exists $F \in C^{m, \omega}\left(\mathbb{R}^{n}\right)$, with

$$
\|F\|_{C^{m, \omega}\left(\mathbb{R}^{n}\right)} \leq C^{\prime} \quad \text { and } \quad J_{x}(F) \in f(x)+C^{\prime} \cdot \sigma(x)
$$

for each $x \in E \cap B\left(y^{0}, c^{\prime}\right)$; here, $C^{\prime}$ and $c^{\prime}$ depend only on $C, m, n$ in (I) and (II).

Once we have the above Local Theorem, we may remove the restriction to finite sets E, by a compactness argument using Ascoli's Theorem. We may then use a partition of unity to pass from a local to a global result, completing the proof of Theorem 2. 


\section{Starting the Main Induction}

In this section, we give the proof of Lemma 5.1. We will show that the Strong Main Lemma holds for $\mathcal{A}=\mathcal{M}$. The Weak Main Lemma for $\mathcal{A}=\mathcal{M}$ then follows at once.

Let $C, \bar{a}_{0}, \omega, E, f, \sigma, y^{0},\left(P_{\alpha}\right)_{\alpha \in \mathcal{M}}$ satisfy $(\mathrm{SL0}, \ldots, 5)$ with $\mathcal{A}=\mathcal{M}$ and $k^{\#}=1$. We must produce $F \in C^{m, \omega}\left(\mathbb{R}^{n}\right)$ satisfying (SL6,7). We will show that (SL6,7) hold with $F=0$. To see this we argue as follows.

We write $c_{1}, C_{1}, C^{\prime}$, etc., to denote constants determined by $C, m, n$ in $(\mathrm{SL} 0, \ldots, 5)$. We introduce a small enough constant $\delta>0$, to be picked later, and we assume that

$$
\bar{a}_{0}<\delta
$$

Now suppose we are given

$$
x^{\prime} \in E \cap B\left(y^{0}, \delta\right) .
$$

Taking $S=\left\{x^{\prime}\right\}$ in (SL3), we obtain, for each $\alpha \in \mathcal{M}$, a function $\varphi_{\alpha} \in$ $C_{\ell o c}^{m, \omega}\left(\mathbb{R}^{n}\right)$, with

$$
\left|\partial^{\beta} \varphi_{\alpha}(x)-\partial^{\beta} \varphi_{\alpha}(y)\right| \leq \bar{a}_{0} \omega(|x-y|)+C|x-y|
$$

for $|\beta|=m, x, y \in \mathbb{R}^{n},|x-y| \leq 1$;

$$
\begin{aligned}
& J_{x^{\prime}}\left(\varphi_{\alpha}\right) \in C \sigma\left(x^{\prime}\right) ; \quad \text { and } \\
& J_{y^{0}}\left(\varphi_{\alpha}\right)=P_{\alpha} .
\end{aligned}
$$
gives

$$
\partial^{\beta} \varphi_{\alpha}\left(y^{0}\right)=\delta_{\beta \alpha} \quad \text { for } \beta, \alpha \in \mathcal{M} \text {. }
$$

Since $\omega(t) \leq 1$ for $t \in[0,1]$, we obtain from (1), (3) that

$$
\left|\partial^{\beta} \varphi_{\alpha}(x)-\partial^{\beta} \varphi_{\alpha}\left(y^{0}\right)\right| \leq C_{1} \delta \quad \text { for all } x \in B\left(y^{0}, \delta\right) \text {, if }|\beta|=m \text {. }
$$

By downward induction on $|\beta|$, we show that (7) holds for $|\beta| \leq m$. We have just proven (7) for $|\beta|=m$. For the induction step, suppose $|\beta|<m$, and suppose (7) holds for multi-indices of order $|\beta|+1$.

Then we have

$$
\left|\nabla \partial^{\beta} \varphi_{\alpha}(\tilde{x})-\nabla \partial^{\beta} \varphi_{\alpha}\left(y^{0}\right)\right| \leq C_{2} \delta \quad \text { for all } \tilde{x} \in B\left(y^{0}, \delta\right) \text {. }
$$


On the other hand, for $x \in B\left(y^{0}, \delta\right)$, the mean value theorem produces an $\tilde{x}$ on the line segment joining $y^{0}$ to $x$, for which we have

$$
\begin{aligned}
& \partial^{\beta} \varphi_{\alpha}(x)-\partial^{\beta} \varphi_{\alpha}\left(y^{0}\right)=\nabla \partial^{\beta} \varphi_{\alpha}(\tilde{x}) \cdot\left(x-y^{0}\right) \\
& \quad=\left[\nabla \partial^{\beta} \varphi_{\alpha}(\tilde{x})-\nabla \partial^{\beta} \varphi_{\alpha}\left(y^{0}\right)\right] \cdot\left(x-y^{0}\right)+\nabla \partial^{\beta} \varphi_{\alpha}\left(y^{0}\right) \cdot\left(x-y^{0}\right) .
\end{aligned}
$$

From (6) we have at once

$$
\left|\nabla \partial^{\beta} \varphi_{\alpha}\left(y^{0}\right)\right| \leq C_{3} .
$$

Putting (8) and (10) into (9), and recalling that $\left|x-y^{0}\right| \leq \delta$, we find that $\left|\partial^{\beta} \varphi_{\alpha}(x)-\partial^{\beta} \varphi_{\alpha}\left(y^{0}\right)\right| \leq C_{2} \delta^{2}+C_{3} \delta \leq C_{4} \delta$, provided $\delta \leq 1$. This completes the downward induction, proving (7) with a constant that may depend on $|\beta|$. Since $0 \leq|\beta| \leq m$, we conclude that (7) holds with a constant depending only on $C, m, n$ in $(\mathrm{SL} 0, \ldots, 5)$.

From (2), (6), (7), we have

$$
\left|\partial^{\beta} \varphi_{\alpha}\left(x^{\prime}\right)-\delta_{\beta \alpha}\right| \leq C_{5} \delta \quad \text { for all } \beta, \alpha \in \mathcal{M} .
$$

Together with (4), and the fact that $\sigma\left(x^{\prime}\right)$ is convex and symmetric about $0,(11)$ shows that

(12) Given any $P \in \mathcal{P}$, if $\left|\partial^{\beta} P\left(x^{\prime}\right)\right| \leq 1$ for $|\beta| \leq m$, then $P \in C_{6} \cdot \sigma\left(x^{\prime}\right)$, provided we take

(13) $\delta<c_{7}$.

Next, we apply hypothesis (SL4), with $S=\left\{x^{\prime}\right\}$. Thus, we obtain $F^{S} \in$ $C^{m, \omega}\left(\mathbb{R}^{n}\right)$ satisfying in particular

$$
\begin{aligned}
\left|\partial^{\beta} F^{S}\left(x^{\prime}\right)\right| & \leq C(|\beta| \leq m) \quad \text { and } \\
J_{x^{\prime}}\left(F^{S}\right) & \in f\left(x^{\prime}\right)+C \sigma\left(x^{\prime}\right) .
\end{aligned}
$$

From (12) and (14), we see that $J_{x^{\prime}}\left(F^{S}\right) \in C_{8} \cdot \sigma\left(x^{\prime}\right)$, and therefore (15) shows that

$$
f\left(x^{\prime}\right) \in C_{9} \cdot \sigma\left(x^{\prime}\right) .
$$

(We have again used the hypothesis that $\sigma\left(x^{\prime}\right)$ is convex and symmetric about 0 ). Thus, if assumption (1) holds, and if $\delta$ is taken small enough that the above arguments work, then we have shown that every $x^{\prime} \in E \cap B\left(y^{0}, \delta\right)$ satisfies (16).

We may take $\delta$ to be a small enough constant $c^{\prime}$, determined by $C, m, n$ in $(\mathrm{SL0}, \ldots, 5)$. If $c^{\prime}$ is taken small enough, then the above arguments work. Moreover, with $\delta=c^{\prime}$, our assumption (1) follows from hypothesis (SL5). Thus, we have (16) for all $x^{\prime} \in B\left(y^{0}, c^{\prime}\right) \cap E$. This implies immediately that the function $F=0$ satisfies (SL6,7). The proof of Lemma 5.1 is complete. 


\section{Non-Monotonic Sets}

In this section, we prove Lemma 5.2 in the easy case of non-monotonic $\mathcal{A}$.

Lemma 7.1 Fix a non-monotonic set $\mathcal{A} \subset \mathcal{M}$, and assume that the Strong Main Lemma holds for all $\overline{\mathcal{A}}<\mathcal{A}$. Then the Weak Main Lemma holds for $\mathcal{A}$.

Proof: Suppose $\mathcal{A}$ is non-monotonic, and let $C, a_{0}, \omega, E, f, \sigma, y^{0},\left(P_{\alpha}\right)_{\alpha \in \mathcal{A}}$ satisfy $(\mathrm{WL} 0, \ldots, 5)$. We must show that there exist $C^{\prime}, c^{\prime}$ depending only on $C, m, n$, and that there exists $F \in C^{m, \omega}\left(\mathbb{R}^{n}\right)$ satisfying (WL6,7) for those $C^{\prime}$ and $c^{\prime}$. We write $c_{1}, C_{2}$, etc., for constants depending only on $C, m, n$. We call $c_{1}, C_{2}$, etc. "controlled constants".

Since $\mathcal{A}$ is not monotonic, there exist multi-indices $\bar{\alpha}, \bar{\gamma}$, with

$$
\bar{\alpha} \in \mathcal{A}, \quad \bar{\alpha}+\bar{\gamma} \in \mathcal{M} \backslash \mathcal{A} .
$$

We set

$$
\overline{\mathcal{A}}=\mathcal{A} \cup\{\bar{\alpha}+\bar{\gamma}\}
$$

and take $k^{\#}$ as in the Strong Main Lemma for $\overline{\mathcal{A}}$. Note that $\overline{\mathcal{A}}<\mathcal{A}$, by Lemma 3.2 and (1).

Define

$$
P_{\bar{\alpha}+\bar{\gamma}}(x)=\frac{\bar{\alpha} !}{(\bar{\alpha}+\bar{\gamma}) !} \cdot \sum_{|\bar{\beta}| \leq m-|\bar{\gamma}|}\left(\frac{1}{\bar{\beta} !} \partial^{\bar{\beta}} P_{\bar{\alpha}}\left(y^{0}\right)\right) \cdot\left(x-y^{0}\right)^{\bar{\beta}+\bar{\gamma}} .
$$

Thus, $P_{\alpha} \in \mathcal{P}$ is defined for all $\alpha \in \overline{\mathcal{A}}$.

From (3) we obtain easily that

$\partial^{\beta} P_{\bar{\alpha}+\bar{\gamma}}\left(y^{0}\right)=\left[\begin{array}{l}\frac{\bar{\alpha} !}{(\bar{\alpha}+\bar{\gamma}) !} \cdot \frac{(\bar{\beta}+\bar{\gamma}) !}{\bar{\beta} !} \cdot\left(\partial^{\bar{\beta}} P_{\bar{\alpha}}\left(y^{0}\right)\right) \text { if } \beta=\bar{\beta}+\bar{\gamma} \text { for some } \bar{\beta} \\ 0 \text { if } \beta \text { doesn't have the form } \bar{\beta}+\bar{\gamma} \text { for a multi-index } \bar{\beta}\end{array}\right]$.

Consequently, (WL2) gives

$$
\left|\partial^{\beta} P_{\bar{\alpha}+\bar{\gamma}}\left(y^{0}\right)-\delta_{\beta, \bar{\alpha}+\bar{\gamma}}\right| \leq C_{1} a_{0} \quad \text { for all } \beta \in \mathcal{M} .
$$

From (4) and another application of (WL2), we see that

$$
\left|\partial^{\beta} P_{\alpha}\left(y^{0}\right)-\delta_{\beta \alpha}\right| \leq C_{1} a_{0} \quad \text { for all } \alpha \in \overline{\mathcal{A}}, \beta \in \mathcal{M} .
$$

From (5) and (WL5), we see that the matrix $\left(\partial^{\beta} P_{\alpha}\left(y^{0}\right)\right)_{\beta, \alpha \in \overline{\mathcal{A}}}$ is invertible, and its inverse matrix $\left(M_{\alpha^{\prime} \alpha}\right)_{\alpha^{\prime}, \alpha \in \overline{\mathcal{A}}}$ satisfies

$$
\left|M_{\alpha^{\prime} \alpha}\right| \leq C_{2} \quad \text { for all } \alpha^{\prime}, \alpha \in \overline{\mathcal{A}} .
$$


By definition of $\left(M_{\alpha^{\prime} \alpha}\right)$, we have

$$
\delta_{\beta \alpha}=\sum_{\alpha^{\prime} \in \overline{\mathcal{A}}} \partial^{\beta} P_{\alpha^{\prime}}\left(y^{0}\right) \cdot M_{\alpha^{\prime} \alpha} \quad \text { for all } \beta, \alpha \in \overline{\mathcal{A}}
$$

We define

$$
\bar{P}_{\alpha}=\sum_{\alpha^{\prime} \in \overline{\mathcal{A}}} P_{\alpha^{\prime}} \cdot M_{\alpha^{\prime} \alpha} \quad \text { for all } \alpha \in \overline{\mathcal{A}}
$$

Thus, $\bar{P}_{\alpha} \in \mathcal{P}$ for $\alpha \in \overline{\mathcal{A}}$, and, from (7), (8) we have

$$
\partial^{\beta} \bar{P}_{\alpha}\left(y^{0}\right)=\delta_{\beta \alpha} \quad \text { for all } \beta, \alpha \in \overline{\mathcal{A}} \text {. }
$$

Also, from (5), (6), (8) and (WL5), we have

$$
\left|\partial^{\beta} \bar{P}_{\alpha}\left(y^{0}\right)\right| \leq C_{3} \quad \text { for all } \beta \in \mathcal{M}, \alpha \in \overline{\mathcal{A}}
$$

Next, let $S \subset E$ be given, with $\#(S) \leq k^{\#}$. For $\alpha \in \mathcal{A}$, we let $\varphi_{\alpha}^{S} \in$ $C_{\ell o c}^{m, \omega}\left(\mathbb{R}^{n}\right)$ be as in (WL3). We define also

$$
\varphi_{\bar{\alpha}+\bar{\gamma}}^{S}(x)=\frac{\bar{\alpha} !}{(\bar{\alpha}+\bar{\gamma}) !}\left(x-y^{0}\right)^{\bar{\gamma}} \cdot \chi\left(x-y^{0}\right) \cdot \varphi_{\bar{\alpha}}^{S}(x) \quad \text { on } \mathbb{R}^{n},
$$

where

(12) $\|\chi\|_{C^{m+1}\left(\mathbb{R}^{n}\right)} \leq C_{4}, \quad \chi=1 \quad$ on $B(0,1 / 20), \quad \operatorname{supp} \chi \subset B(0,1 / 10)$.

We prepare to estimate the derivatives of $\varphi_{\bar{\alpha}+\bar{\gamma}}^{S}$. From (WL3)(a) and the fact that $\omega(t) \leq 1$ for $t \in[0,1]$ (since $\omega$ is a regular modulus of continuity), we have

$$
\left|\partial^{\beta} \varphi_{\bar{\alpha}}^{S}(x)-\partial^{\beta} \varphi_{\bar{\alpha}}^{S}\left(y^{0}\right)\right| \leq a_{0} \text { for } x \in B\left(y^{0}, 1\right) \text { and }|\beta|=m .
$$

Also, from (WL2), (WL3)(c), (WL5), we have

$$
\left|\partial^{\beta} \varphi_{\bar{\alpha}}^{S}\left(y^{0}\right)\right| \leq C_{5} \text { for }|\beta| \leq m .
$$

Consequently,

$$
\left|\partial^{\beta} \varphi_{\bar{\alpha}}^{S}(x)\right| \leq C_{6} \quad \text { for } x \in B\left(y^{0}, 1\right) \text { and }|\beta| \leq m .
$$

From (11), (12), (13), we see that

$$
\left|\partial^{\beta} \varphi_{\bar{\alpha}+\bar{\gamma}}^{S}(x)\right| \leq C_{7} \quad \text { for } x \in \mathbb{R}^{n},|\beta| \leq m .
$$


Next, we prepare to estimate the modulus of continuity of $\partial^{\beta} \varphi_{\bar{\alpha}+\bar{\gamma}}^{S}$ for $|\beta|=m$. Set

$$
\tilde{\chi}(x)=\frac{\bar{\alpha} !}{(\bar{\alpha}+\bar{\gamma}) !}\left(x-y^{0}\right)^{\gamma} \cdot \chi\left(x-y^{0}\right) .
$$

Thus, (11), (12) give

$$
\begin{aligned}
\varphi_{\bar{\alpha}+\bar{\gamma}}^{S} & =\tilde{\chi} \cdot \varphi_{\bar{\alpha}}^{S}, \quad \text { and } \\
\|\tilde{\chi}\|_{C^{m+1}\left(\mathbb{R}^{n}\right)} & \leq C_{8}, \quad \operatorname{supp} \tilde{\chi} \subset B\left(y^{0}, 1 / 10\right) .
\end{aligned}
$$

Since $\tilde{\chi}, \varphi_{\bar{\alpha}}^{S} \in C_{\ell o c}^{m}\left(\mathbb{R}^{n}\right)$, we know that, for $|\beta|=m$, we have

$$
\begin{aligned}
& \partial^{\beta} \varphi_{\bar{\alpha}+\bar{\gamma}}^{S}(x)-\partial^{\beta} \varphi_{\bar{\alpha}+\bar{\gamma}}^{S}(y)= \\
& =\sum_{\beta^{\prime}+\beta^{\prime \prime}=\beta} c\left(\beta^{\prime}, \beta^{\prime \prime}\right)\left[\partial^{\beta^{\prime}} \tilde{\chi}(x) \cdot \partial^{\beta^{\prime \prime}} \varphi_{\bar{\alpha}}^{S}(x)-\partial^{\beta^{\prime}} \tilde{\chi}(y) \cdot \partial^{\beta^{\prime \prime}} \varphi_{\bar{\alpha}}^{S}(y)\right] \\
& =\tilde{\chi}(x) \cdot\left[\partial^{\beta} \varphi_{\bar{\alpha}}^{S}(x)-\partial^{\beta} \varphi_{\bar{\alpha}}^{S}(y)\right]+[\tilde{\chi}(x)-\tilde{\chi}(y)] \cdot \partial^{\beta} \varphi_{\bar{\alpha}}^{S}(y) \\
& \quad+\sum_{\substack{\beta^{\prime}+\beta^{\prime \prime}=\beta \\
\beta^{\prime} \neq 0}} c\left(\beta, \beta^{\prime}\right)\left(\partial^{\beta^{\prime}} \tilde{\chi}(x)\right) \cdot\left[\partial^{\beta^{\prime \prime}} \varphi_{\bar{\alpha}}^{S}(x)-\partial^{\beta^{\prime \prime}} \varphi_{\bar{\alpha}}^{S}(y)\right] \\
& \quad+\sum_{\substack{\beta^{\prime}+\beta^{\prime \prime}=\beta \\
\beta^{\prime} \neq 0}} c\left(\beta, \beta^{\prime}\right)\left[\partial^{\beta^{\prime}} \tilde{\chi}(x)-\partial^{\beta^{\prime}} \tilde{\chi}(y)\right] \cdot\left(\partial^{\beta^{\prime \prime}} \varphi_{\bar{\alpha}}^{S}(y)\right) .
\end{aligned}
$$

Suppose $x, y \in B\left(y^{0}, 1\right)$ and $|x-y| \leq 1 / 2$. Then, by virtue of (13) and (16), the last two sums on the right in (17) have absolute values less than or equal to $C_{9} \cdot|x-y|$. Also, from (13) and (16), we have $\left|[\tilde{\chi}(x)-\tilde{\chi}(y)] \cdot \partial^{\beta} \varphi_{\bar{\alpha}}^{S}(y)\right| \leq$ $C_{10} \cdot|x-y|$. Hence, (17) shows that

(18) $\left|\partial^{\beta} \varphi_{\bar{\alpha}+\bar{\gamma}}^{S}(x)-\partial^{\beta} \varphi_{\bar{\alpha}+\bar{\gamma}}^{S}(y)\right| \leq\left|\tilde{\chi}(x) \cdot\left[\partial^{\beta} \varphi_{\bar{\alpha}}^{S}(x)-\partial^{\beta} \varphi_{\bar{\alpha}}^{S}(y)\right]\right|+C_{11} \cdot|x-y|$

for $x, y \in B\left(y^{0}, 1\right),|x-y| \leq 1 / 2$.

Putting (WL3)(a) and (16) into (18), we learn that

$$
\left|\partial^{\beta} \varphi_{\bar{\alpha}+\bar{\gamma}}^{S}(x)-\partial^{\beta} \varphi_{\bar{\alpha}+\bar{\gamma}}^{S}(y)\right| \leq C_{12} a_{0} \omega(|x-y|)+C_{12}|x-y|
$$

for $x, y \in B\left(y^{0}, 1\right),|x-y| \leq 1 / 2,|\beta|=m$.

On the other hand, if $|x-y| \leq 1 / 2$ and $x$ or $y$ lies outside $B\left(y^{0}, 1\right)$, then we have $\left|x-y^{0}\right|,\left|y-y^{0}\right| \geq 1 / 2$, and therefore $\partial^{\beta} \varphi_{\bar{\alpha}+\bar{\gamma}}^{S}(x)=\partial^{\beta} \varphi_{\bar{\alpha}+\bar{\gamma}}^{S}(y)=0$, by (15), (16). Hence, the hypothesis $x, y \in B\left(y^{0}, 1\right)$ may be dropped from (19). Thus, we have

$$
\left|\partial^{\beta} \varphi_{\bar{\alpha}+\bar{\gamma}}^{S}(x)-\partial^{\beta} \varphi_{\bar{\alpha}+\bar{\gamma}}^{S}(y)\right| \leq C_{12} a_{0} \omega(|x-y|)+C_{12}|x-y|
$$

for $x, y \in \mathbb{R}^{n},|x-y| \leq 1 / 2,|\beta|=m$. 
Also, for $1 / 2 \leq|x-y| \leq 1$, we see from (14) that

$\left|\partial^{\beta} \varphi_{\bar{\alpha}+\bar{\gamma}}^{S}(x)-\partial^{\beta} \varphi_{\bar{\alpha}+\bar{\gamma}}^{S}(y)\right| \leq\left|\partial^{\beta} \varphi_{\bar{\alpha}+\bar{\gamma}}^{S}(x)\right|+\left|\partial^{\beta} \varphi_{\bar{\alpha}+\bar{\gamma}}^{S}(y)\right| \leq C_{13} \leq 2 C_{13}|x-y|$.

Together with (20), this implies that

(21) $\left|\partial^{\beta} \varphi_{\bar{\alpha}+\bar{\gamma}}^{S}(x)-\partial^{\beta} \varphi_{\bar{\alpha}+\bar{\gamma}}^{S}(y)\right| \leq C_{14} a_{0} \omega(|x-y|)+C_{14}|x-y|$ for $x, y \in \mathbb{R}^{n}$, $|x-y| \leq 1,|\beta|=m$. In particular, $\varphi_{\bar{\alpha}+\bar{\gamma}}^{S} \in C^{m, \omega}\left(\mathbb{R}^{n}\right)$, thanks to (14), $(21)$, and the estimate $t \leq \omega(t)$ valid on $[0,1]$ for a regular modulus of continuity.

From (WL3)(a) and (21), we conclude that

$$
\left|\partial^{\beta} \varphi_{\alpha}^{S}(x)-\partial^{\beta} \varphi_{\alpha}^{S}(y)\right| \leq C_{15} a_{0} \omega(|x-y|)+C_{15}|x-y|
$$

for $|x-y| \leq 1, \alpha \in \overline{\mathcal{A}},|\beta|=m$.

At last, we have estimated the modulus of continuity of the $m^{\text {th }}$ derivatives of the $\varphi_{\alpha}^{S}(\alpha \in \overline{\mathcal{A}})$. In particular, we have $\varphi_{\alpha}^{S} \in C_{\ell o c}^{m, \omega}\left(\mathbb{R}^{n}\right)$ for $\alpha \in \overline{\mathcal{A}}$.

Next, suppose $x \in S \cap B\left(y^{0}, 1\right)$.

From (13), (16) and (WL3)(b), we have

$$
\left|\partial^{\beta}\left(c_{16} \varphi_{\bar{\alpha}}\right)(x)\right|,\left|\partial^{\beta}\left(c_{16} \tilde{\chi}\right)(x)\right| \leq 1 \quad \text { for }|\beta| \leq m \text {; and } \quad J_{x}\left(c_{16} \varphi_{\bar{\alpha}}\right) \in \sigma(x) .
$$

Taking $P=c_{16} J_{x}\left(\varphi_{\bar{\alpha}}\right), Q=c_{16} J_{x}(\tilde{\chi})$, and $\delta=1$ in the definition of Whitney $\omega$-convexity, we conclude that $J_{x}(\tilde{\chi}) \cdot J_{x}\left(\varphi_{\bar{\alpha}}\right) \in C_{17} \sigma(x)$, where the multiplication is taken in $\mathcal{R}_{x}$.

Together with (15), this shows that

$$
J_{x}\left(\varphi_{\bar{\alpha}+\bar{\gamma}}^{S}\right) \in C_{17} \sigma(x) \text { for } x \in S \cap B\left(y^{0}, 1\right) .
$$

On the other hand, for $x \in S \backslash B\left(y^{0}, 1\right)$, we have $J_{x}\left(\varphi_{\bar{\alpha}+\bar{\gamma}}^{S}\right)=0$ by (15), (16); and therefore $J_{x}\left(\varphi_{\bar{\alpha}+\bar{\gamma}}^{S}\right) \in C_{17} \sigma(x)$ since $\sigma(x)$ is convex and symmetric about the origin. Thus,

$$
J_{x}\left(\varphi_{\bar{\alpha}+\bar{\gamma}}^{S}\right) \in C_{17} \sigma(x) \text { for all } x \in S .
$$

Together with (WL3)(b), this shows that

$$
J_{x}\left(\varphi_{\alpha}^{S}\right) \in C_{18} \sigma(x) \quad \text { for all } x \in S, \alpha \in \overline{\mathcal{A}} .
$$

Also, from (11), (12), and (WL3)(c), we have

$$
\varphi_{\bar{\alpha}+\bar{\gamma}}^{S}(x)-\frac{\bar{\alpha} !}{(\bar{\alpha}+\bar{\gamma}) !}\left(x-y^{0}\right)^{\bar{\gamma}} P_{\bar{\alpha}}(x)=o\left(|x-y|^{m}\right) \text { as } x \longrightarrow y^{0} \text {. }
$$


On the other hand, (3) shows that

$$
P_{\bar{\alpha}+\bar{\gamma}}(x)-\frac{\bar{\alpha} !}{(\bar{\alpha}+\bar{\gamma}) !}\left(x-y^{0}\right)^{\bar{\gamma}} P_{\bar{\alpha}}(x)=o\left(\left|x-y^{0}\right|^{m}\right) \text { as } x \longrightarrow y^{0} .
$$

Hence,

$$
\varphi_{\bar{\alpha}+\bar{\gamma}}^{S}(x)-P_{\bar{\alpha}+\bar{\gamma}}(x)=o\left(\left|x-y^{0}\right|^{m}\right) \text { as } x \longrightarrow y^{0} .
$$

Since also $P_{\bar{\alpha}+\bar{\gamma}} \in \mathcal{P}$ and $\varphi_{\bar{\alpha}+\bar{\gamma}}^{S} \in C^{m}\left(\mathbb{R}^{n}\right)$, we have

$$
J_{y^{0}}\left(\varphi_{\bar{\alpha}+\bar{\gamma}}^{S}\right)=P_{\bar{\alpha}+\bar{\gamma}}
$$

Together with (WL3)(c), this shows that

$$
J_{y^{0}}\left(\varphi_{\alpha}^{S}\right)=P_{\alpha} \quad \text { for all } \alpha \in \overline{\mathcal{A}}
$$

Thus, the $\left(\varphi_{\alpha}^{S}\right)_{\alpha \in \overline{\mathcal{A}}}$ satisfy (22), (23), (24).

Next, given $S \subset E$ with $\#(S) \leq k^{\#}$, let the $\varphi_{\alpha}^{S}(\alpha \in \overline{\mathcal{A}})$ be as above, and define

$$
\bar{\varphi}_{\alpha}^{S}=\sum_{\alpha^{\prime} \in \overline{\mathcal{A}}} \varphi_{\alpha^{\prime}}^{S} \cdot M_{\alpha^{\prime} \alpha} \quad \text { for all } \alpha \in \overline{\mathcal{A}}
$$

Thus, $\bar{\varphi}_{\alpha}^{S} \in C_{\ell o c}^{m, \omega}\left(\mathbb{R}^{n}\right)$, for all $\alpha \in \overline{\mathcal{A}}$.

From (6), (22), (23), we have

$$
\left|\partial^{\beta} \bar{\varphi}_{\alpha}^{S}(x)-\partial^{\beta} \bar{\varphi}_{\alpha}^{S}(y)\right| \leq C_{19} a_{0} \omega(|x-y|)+C_{19}|x-y|
$$

for $x, y \in \mathbb{R}^{n},|x-y| \leq 1,|\beta|=m, \alpha \in \overline{\mathcal{A}}$ and

$$
J_{x}\left(\bar{\varphi}_{\alpha}^{S}\right) \in C_{20} \cdot \sigma(x) \quad \text { for all } x \in S, \alpha \in \overline{\mathcal{A}} .
$$

(We use the fact that $\sigma(x)$ is convex and symmetric about the origin to prove (27).)

Also, comparing (8) with (25), and recalling (24), we see that

$$
J_{y^{0}}\left(\bar{\varphi}_{\alpha}^{S}\right)=\bar{P}_{\alpha} \quad \text { for all } \alpha \in \overline{\mathcal{A}}
$$

Next, we check that the hypotheses of the Strong Main Lemma for $\overline{\mathcal{A}}$ are satisfied by $C_{21}, \bar{a}_{0}, E, f, \sigma, y^{0}, \omega, \bar{P}_{\alpha}(\alpha \in \overline{\mathcal{A}})$, provided $C_{21}$ is a large enough controlled constant, and $\bar{a}_{0}$ is a small enough constant determined by $C_{21}, m, n$. In fact, $C_{21}$ and $\bar{a}_{0}$ are constants; $\omega$ is a regular modulus of continuity; $E \subset \mathbb{R}^{n}$ is a finite set; $y^{0} \in \mathbb{R}^{n}$; and $\bar{P}_{\alpha} \in \mathcal{P}$ for all $\alpha \in \overline{\mathcal{A}}$. 
Also, for each $x \in E, f(x) \in \mathcal{R}_{x}$ is an $m$-jet, and $\sigma(x)$ is Whitney $\omega$-convex with Whitney constant $C$ (hence also with Whitney constant $\left.C_{21}>C\right)$.

Thus, (SL0) holds. From (9) we see that (SL1) holds.

Taking $C_{21}>C_{3}$, we see from (10) that (SL2) holds, even without the restriction to $\beta \geq \alpha$. To see that (SL3) holds, we let $\alpha \in \overline{\mathcal{A}}$ and $S \subset E$, with $\#(S) \leq k^{\#}$. Let $\bar{\varphi}_{\alpha}^{S}$ be as in (26), (27), (28). Thus, $\bar{\varphi}_{\alpha}^{S} \in C_{\ell o c}^{m, \omega}\left(\mathbb{R}^{n}\right)$, and (26), (27), (28) imply (SL3)(a),(b),(c), provided we take $C_{21}>C_{19}$, $C_{21}>C_{20}$, and provided we have

$$
C_{19} \cdot a_{0}<\bar{a}_{0}
$$

However, (29) follows from hypothesis (WL5), since we are taking $\bar{a}_{0}$ to be a small enough constant determined by $C_{21}$ and $m, n$. (In fact, since $C_{21}$ is a controlled constant, so is $\bar{a}_{0}$, and therefore, (29) just says that $a_{0}$ is less than a certain controlled constant.) This shows that (SL3) holds.

Also, (SL4) follows from our hypothesis (WL4), provided we take $C_{21}>C$. Finally, (SL5) holds here, since we picked $\bar{a}_{0}$ to be a small enough constant, determined by $C_{21}, m, n$.

This completes the verification of the hypotheses of the Strong Main Lemma for $\overline{\mathcal{A}}$, for $C_{21}, \bar{a}_{0}, \omega, E, f, \sigma, y^{0},\left(\bar{P}_{\alpha}\right)_{\alpha \in \overline{\mathcal{A}}}$.

Since $\overline{\mathcal{A}}<\mathcal{A}$, we are assuming that the Strong Main Lemma holds for $\overline{\mathcal{A}}$. Applying that Lemma, we obtain $F \in C^{m, \omega}\left(\mathbb{R}^{n}\right)$, with

$$
\|F\|_{C^{m, \omega}\left(\mathbb{R}^{n}\right)} \leq C^{\prime}
$$

and

$$
J_{x}(F) \in f(x)+C^{\prime} \sigma(x) \text { for all } x \in E \cap B\left(y^{0}, c^{\prime}\right)
$$

with

$$
C^{\prime} \text { and } c^{\prime} \text { determined by } C_{21}, m, n \text {. }
$$

Since $C_{21}$ is a controlled constant, (32) shows that $C^{\prime}$ and $c^{\prime}$ are also controlled constants. Hence, (30), (31) are the conclusions (WL6,7) of the Weak Main Lemma.

Thus, the Weak Lemma holds for $\mathcal{A}$.

The proof of Lemma 7.1 is complete. 


\section{A Consequence of the Main Inductive Assumption}

In this section, we establish the following result.

Lemma 8.1 Fix $\mathcal{A} \subset \mathcal{M}$, and assume that the Strong Main Lemma holds for all $\overline{\mathcal{A}}<\mathcal{A}$. Then there exists $k_{\text {old }}^{\#}$, depending only on $m$ and $n$, and there exists a function $A \mapsto a_{0}^{\text {old }}(A)$ mapping $(0, \infty) \longrightarrow(0, \infty)$, for which the following holds.

Let $A>0$ be given. Let $Q \subset \mathbb{R}^{n}$ be a cube of diameter $\leq 1, \omega$ a regular modulus of continuity, $E$ a finite subset of $\mathbb{R}^{n}$. Suppose that, for each $x \in E$, we are given an $m$-jet $f(x) \in \mathcal{R}_{x}$ and a subset $\sigma(x) \subset \mathcal{R}_{x}$.

Suppose also that, for each $y \in Q^{* *}$, we are given a set $\overline{\mathcal{A}}^{y}<\mathcal{A}$, and a family of polynomials $\bar{P}_{\alpha}^{y} \in \mathcal{P}$, indexed by $\alpha \in \overline{\mathcal{A}}^{y}$.

Assume that the following conditions are satisfied.

(G0) For each $x \in E$, the set $\sigma(x)$ is Whitney $\omega$-convex, with Whitney constant $A$.

(G1) $\partial^{\beta} \bar{P}_{\alpha}^{y}(y)=\delta_{\beta \alpha}$ for all $\beta, \alpha \in \overline{\mathcal{A}}^{y}, y \in Q^{* *}$.

(G2) $\left|\partial^{\beta} \bar{P}_{\alpha}^{y}(y)\right| \leq A \delta_{Q}^{|\alpha|-|\beta|}$ for all $\beta \in \mathcal{M}, \alpha \in \overline{\mathcal{A}}^{y}, y \in Q^{* *}$ with $\beta \geq \alpha$.

(G3) Given $S \subset E$ with $\#(S) \leq k_{\text {old }}^{\#}$, and given $y \in Q^{* *}$ and $\alpha \in \overline{\mathcal{A}}^{y}$, there exists $\varphi_{\alpha}^{S} \in C_{\ell o c}^{m, \omega}\left(\mathbb{R}^{n}\right)$, with

(a) $\left|\partial^{\beta} \varphi_{\alpha}^{S}\left(x^{\prime}\right)-\partial^{\beta} \varphi_{\alpha}^{S}\left(x^{\prime \prime}\right)\right| \leq A \delta_{Q}^{|\alpha|-m-1} \cdot\left|x^{\prime}-x^{\prime \prime}\right|+a_{0}^{o l d}(A) \cdot \delta_{Q}^{|\alpha|-m} \cdot \frac{\omega\left(\left|x^{\prime}-x^{\prime \prime}\right|\right)}{\omega\left(\delta_{Q}\right)}$ for $\left|x^{\prime}-x^{\prime \prime}\right| \leq \delta_{Q}$ and $|\beta|=m$;

(b) $J_{x}\left(\varphi_{\alpha}^{S}\right) \in A \frac{\delta_{Q}^{|\alpha|-m}}{\omega\left(\delta_{Q}\right)} \cdot \sigma(x)$ for all $x \in S$;

(c) $J_{y}\left(\varphi_{\alpha}^{S}\right)=\bar{P}_{\alpha}^{y}$.

(G4) Given $S \subset E$ with $\#(S) \leq k_{\text {old }}^{\#}$ there exists $F^{S} \in C^{m, \omega}\left(\mathbb{R}^{n}\right)$, with

(a) $\left\|\partial^{\beta} F^{S}\right\|_{C^{0}\left(\mathbb{R}^{n}\right)} \leq A \cdot \omega\left(\delta_{Q}\right) \cdot \delta_{Q}^{m-|\beta|}$ for $|\beta| \leq m$;

(b) $\left|\partial^{\beta} F^{S}\left(x^{\prime}\right)-\partial^{\beta} F^{S}\left(x^{\prime \prime}\right)\right| \leq A \cdot \omega\left(\left|x^{\prime}-x^{\prime \prime}\right|\right)$ for $|\beta|=m, x^{\prime}, x^{\prime \prime} \in \mathbb{R}^{n}$, $\left|x^{\prime}-x^{\prime \prime}\right| \leq \delta_{Q}$

(c) $J_{x}\left(F^{S}\right) \in f(x)+A \cdot \sigma(x)$ for all $x \in S$. 
Then there exists $F \in C^{m, \omega}\left(\mathbb{R}^{n}\right)$, with

(G5) $\left\|\partial^{\beta} F\right\|_{C^{0}\left(\mathbb{R}^{n}\right)} \leq A^{\prime} \cdot \omega\left(\delta_{Q}\right) \cdot \delta_{Q}^{m-|\beta|}$ for $|\beta| \leq m$;

(G6) $\left|\partial^{\beta} F\left(x^{\prime}\right)-\partial^{\beta} F\left(x^{\prime \prime}\right)\right| \leq A^{\prime} \cdot \omega\left(\left|x^{\prime}-x^{\prime \prime}\right|\right)$ for $|\beta|=m, x^{\prime}, x^{\prime \prime} \in \mathbb{R}^{n}$, $\left|x^{\prime}-x^{\prime \prime}\right| \leq \delta_{Q}$

(G7) $J_{x}(F) \in f(x)+A^{\prime} \cdot \sigma(x)$ for all $x \in E \cap Q^{*}$.

Here, $A^{\prime}$ is determined by $A, m, n$.

Proof: By a rescaling, we may reduce matters to the case $\delta_{Q}=1$. We spell out the details. Let $A, Q, \omega, E, f, \sigma, \overline{\mathcal{A}}^{y},\left(\bar{P}_{\alpha}^{y}\right)_{\alpha \in \overline{\mathcal{A}}^{y}}$ be as in the hypotheses of Lemma 8.1. We set

$$
\begin{aligned}
& \overline{\bar{Q}}=\delta_{Q}^{-1} \cdot Q ; \\
& \overline{\bar{S}}=\delta_{Q}^{-1} \cdot S \quad \text { for } S \subset E \text {; } \\
& \overline{\bar{E}}=\delta_{Q}^{-1} \cdot E \text {; } \\
& \overline{\bar{\omega}}(t)=\left(\omega\left(\delta_{Q}\right)\right)^{-1} \omega\left(\delta_{Q} t\right) \quad \text { for } t \in[0,1] \text {; } \\
& \overline{\bar{y}}=\delta^{-1} y \quad \text { for } y \in Q^{* *} \text {; } \\
& \overline{\bar{P}}_{\alpha}^{\overline{\bar{y}}}(\overline{\bar{x}})=\delta_{Q}^{-|\alpha|} \cdot P_{\alpha}^{y}\left(\delta_{Q} \overline{\bar{x}}\right) \quad \text { for } y \in Q^{* *}, \overline{\bar{x}} \in \mathbb{R}^{n}, \alpha \in \overline{\mathcal{A}}^{y} ; \\
& \overline{\bar{\varphi}}_{\alpha}^{\overline{\bar{S}}}(\overline{\bar{x}})=\delta_{Q}^{-|\alpha|} \varphi_{\alpha}^{S}\left(\delta_{Q} \overline{\bar{x}}\right) \quad \text { for } \overline{\bar{x}} \in \mathbb{R}^{n}, \alpha \in \overline{\mathcal{A}}^{y}, y \in Q^{* *} \text {; } \\
& \overline{\bar{f}}(\overline{\bar{x}})=\left(\omega\left(\delta_{Q}\right) \cdot \delta_{Q}^{m}\right)^{-1} \cdot\left[\left(f\left(\delta_{Q} \overline{\bar{x}}\right)\right) \circ \tau\right], \quad \text { for } \overline{\bar{x}} \in \overline{\bar{E}} \text {, where } \\
& \text { (9) } \quad \tau\left(\overline{\bar{x}}^{\prime}\right)=\delta_{Q} \overline{\bar{x}}^{\prime} \text { for all } \overline{\bar{x}}{ }^{\prime} \in \mathbb{R}^{n} \text {; } \\
& \overline{\bar{\sigma}}(\overline{\bar{x}})=\left\{\left(\omega\left(\delta_{Q}\right) \cdot \delta_{Q}^{m}\right)^{-1} \cdot[P \circ \tau]: P \in \sigma\left(\delta_{Q} \overline{\bar{x}}\right)\right\} \quad \text { for } \overline{\bar{x}} \in \overline{\bar{E}} \text {; } \\
& \overline{\bar{F}}^{\overline{\bar{S}}}(\overline{\bar{x}})=\left(\omega\left(\delta_{Q}\right) \cdot \delta_{Q}^{m}\right)^{-1} \cdot F^{S}\left(\delta_{Q} \overline{\bar{x}}\right) \quad \text { for } \overline{\bar{x}} \in \mathbb{R}^{n} \text {. } \\
& \overline{\mathcal{A}}^{\overline{\bar{y}}}=\overline{\mathcal{A}}^{y}=\overline{\mathcal{A}}^{\delta_{Q} \overline{\bar{y}}} \text { for } \overline{\bar{y}} \in \overline{\bar{Q}}^{* *} \text {. }
\end{aligned}
$$

Note that (4) makes sense, since we have assumed that $\delta_{Q} \leq 1$, and $\omega$ is defined on $[0,1]$.

We check in detail that

$$
A, \overline{\bar{Q}}, \overline{\bar{\omega}}, \overline{\bar{E}}, \overline{\bar{f}}, \overline{\bar{\sigma}}, \overline{\overline{\mathcal{A}}},\left(\overline{\bar{P}}_{\alpha}^{\overline{\bar{y}}}\right)_{\alpha \in \overline{\overline{\mathcal{A}}}} \overline{\bar{y}}
$$

satisfy the hypotheses of Lemma 8.1 , with $\delta_{\overline{\bar{Q}}}=1$. The verification is as follows: 
Evidently, $A>0 ; \overline{\bar{Q}} \subset \mathbb{R}^{n}$ is a cube of diameter $\leq 1$ (in fact $\delta_{\overline{\bar{Q}}}=1$; see (1)); $\overline{\bar{\omega}}$ is a regular modulus of continuity (see (4) and the definition of a regular modulus of continuity); and $\overline{\bar{E}}$ is a finite subset of $\mathbb{R}^{n}$ (see (3)). Also, for each $\overline{\bar{x}} \in \overline{\bar{E}}$, we have $\delta_{Q} \overline{\bar{x}} \in E$ (see (3)), hence

$$
f\left(\delta_{Q} \overline{\bar{x}}\right) \in \mathcal{R}_{\delta_{Q} \overline{\bar{x}}}=\mathcal{R}_{\tau(\overline{\bar{x}})}
$$

(see (9)), hence $\left[f\left(\delta_{Q} \overline{\bar{x}}\right) \circ \tau\right] \in \mathcal{R}_{\overline{\bar{x}}}$, and thus $\overline{\bar{f}}(\overline{\bar{x}}) \in \mathcal{R}_{\overline{\bar{x}}}$ (see (8)).

Similarly, for each $\overline{\bar{x}} \in \overline{\bar{E}}$, we have $\delta_{Q} \overline{\bar{x}} \in E$ (see (3)), hence

$$
\sigma\left(\delta_{Q} \overline{\bar{x}}\right) \subset \mathcal{R}_{\delta_{Q} \overline{\bar{x}}}=\mathcal{R}_{\tau(\overline{\bar{x}})},
$$

hence $\left\{P \circ \tau: P \in \sigma\left(\delta_{Q} \overline{\bar{x}}\right)\right\} \subset \mathcal{R}_{\overline{\bar{x}}}$, hence $\overline{\bar{\sigma}}(\overline{\bar{x}}) \subset \mathcal{R}_{\overline{\bar{x}}}$ (see $(10)$ ).

For $\overline{\bar{y}} \in \overline{\bar{Q}}^{* *}$, we have $\overline{\mathcal{A}}^{\overline{\bar{y}}}=\overline{\mathcal{A}}^{\delta_{Q} \overline{\bar{y}}}<\mathcal{A}$ since $\delta_{Q} \overline{\bar{y}} \in Q^{* *}$ (see (1), (12)).

Also, for $\overline{\bar{y}} \in \overline{\bar{Q}}^{* *}$, the family of polynomials $\overline{\bar{P}}_{\alpha}^{\overline{\bar{y}}} \in \mathcal{P}$ is indexed by $\alpha \in \overline{\overline{\mathcal{A}}}^{\overline{\bar{y}}}$ (see (1), (5), (6), (12)).

We check that $A, \overline{\bar{Q}}, \overline{\bar{\omega}}, \overline{\bar{E}}, \overline{\bar{f}}, \overline{\bar{\sigma}}, \overline{\overline{\mathcal{A}}}$ satisfy hypotheses $(\mathrm{G} 0), \ldots,(\mathrm{G} 4)$.

We begin with $(\mathrm{G} 0)$. We note first that, given $\overline{\bar{x}} \in \overline{\bar{E}}$, the set $\overline{\bar{\sigma}}(\overline{\bar{x}})$ is closed, convex, and symmetric about the origin. This is obvious from (10) and the corresponding property of $\sigma(x)$, where $x=\delta_{Q} \overline{\bar{x}} \in E$.

Next, suppose we are given

$$
\overline{\bar{x}} \in \overline{\bar{E}}, \quad \overline{\bar{Q}} \in \mathcal{R}_{\overline{\bar{x}}}, \quad \overline{\bar{P}} \in \overline{\bar{\sigma}}(\overline{\bar{x}}) \subset \mathcal{R}_{\overline{\bar{x}}} \quad \text { and } \quad \overline{\bar{\delta}} \leq 1,
$$

with

$$
\left|\partial^{\alpha} \stackrel{\bar{P}}{P}(\overline{\bar{x}})\right| \leq \overline{\bar{\omega}}(\overline{\bar{\delta}}) \cdot \overline{\bar{\delta}}^{m-|\alpha|}, \quad\left|\partial^{\alpha} \overline{\bar{Q}}(\overline{\bar{x}})\right| \leq \overline{\bar{\delta}}^{-|\alpha|} \quad \text { for }|\alpha| \leq m .
$$

Then by definition (10), we have

$$
\stackrel{\bar{P}}{=}=\left(\omega\left(\delta_{Q}\right) \cdot \delta_{Q}^{m}\right)^{-1} \cdot[P \circ \tau]
$$

with

$$
P \in \sigma(x)
$$

where

$$
x=\delta_{Q} \overline{\bar{x}} .
$$


For a suitable polynomial $Q \in \mathcal{R}_{x}$, we have

$$
\stackrel{\bar{Q}}{=}[Q \circ \tau]
$$

Let us estimate the derivatives of $P$ and $Q$. From (15), (18), we have

$$
P=\left(\omega\left(\delta_{Q}\right) \cdot \delta_{Q}^{m}\right) \cdot\left[\overline{\bar{P}} \circ \tau^{-1}\right], \quad \text { and } \quad Q=\left[\overline{\bar{Q}} \circ \tau^{-1}\right] \text {. }
$$

Therefore, (17) and (14) show that

$$
\begin{aligned}
\left|\partial^{\alpha} P(x)\right| & =\left(\omega\left(\delta_{Q}\right) \cdot \delta_{Q}^{m}\right) \cdot\left|\delta_{Q}^{-|\alpha|} \partial^{\alpha} \stackrel{\bar{P}}{P}(\overline{\bar{x}})\right| \\
& \leq \omega\left(\delta_{Q}\right) \cdot \delta_{Q}^{m-|\alpha|} \cdot \overline{\bar{\omega}}(\overline{\bar{\delta}}) \cdot \overline{\bar{\delta}}^{m-|\alpha|} \\
& =\omega\left(\delta_{Q}\right) \cdot \delta_{Q}^{m-|\alpha|} \cdot\left[\left(\omega\left(\delta_{Q}\right)\right)^{-1} \cdot \omega\left(\delta_{Q} \overline{\bar{\delta}}\right)\right] \cdot \overline{\bar{\delta}}^{m-|\alpha|} \\
& =\omega\left(\delta_{Q} \overline{\bar{\delta}}\right) \cdot\left(\delta_{Q} \overline{\bar{\delta}}\right)^{m-|\alpha|}
\end{aligned}
$$

and

$$
\left|\partial^{\alpha} Q(x)\right|=\delta_{Q}^{-|\alpha|}\left|\partial^{\alpha} \overline{\bar{Q}}(\overline{\bar{x}})\right| \leq\left(\delta_{Q} \overline{\bar{\delta}}\right)^{-|\alpha|} .
$$

We have $\delta_{Q} \overline{\bar{\delta}} \leq 1$, since we assumed that $\delta_{Q} \leq 1, \overline{\bar{\delta}} \leq 1$. Moreover, $\sigma(x)$ is Whitney $\omega$-convex, with Whitney constant $A$, by hypothesis (G0)

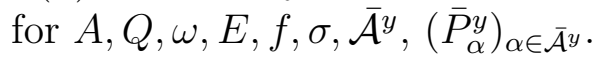

Therefore, (16), (19), (20) imply that

$$
Q \cdot P \in A \sigma(x)
$$

where the multiplication in (21) is taken in $\mathcal{R}_{x}$.

On the other hand, (15) and (18) show that

$$
\overline{\bar{Q}} \cdot \stackrel{\bar{P}}{P}=\left(\omega\left(\delta_{Q}\right) \cdot \delta_{Q}^{m}\right)^{-1} \cdot[(Q \cdot P) \circ \tau] .
$$

Here, $Q \cdot P$ is as in (21), and the multiplication $\overline{\bar{Q}} \cdot \stackrel{\bar{P}}{P}$ is taken in $\mathcal{R}_{\overline{\bar{x}}}$.

From (21) and (22), we see that

$$
A^{-1}(\overline{\bar{Q}} \cdot \overline{\bar{P}}) \in\left\{\left(\omega\left(\delta_{Q}\right) \cdot \delta_{Q}^{m}\right)^{-1} \cdot[\mathcal{S} \circ \tau]: \mathcal{S} \in \sigma(x)\right\}
$$

Comparing this with the definition (10) of $\overline{\bar{\sigma}}(\overline{\bar{x}})$, and recalling (17), we see that $A^{-1}(\overline{\bar{Q}} \cdot \stackrel{\bar{P}}{)} \in \overline{\bar{\sigma}}(\overline{\bar{x}})$. 
Thus, we have shown that (13), (14) imply $\overline{\bar{Q}} \cdot \overline{\bar{P}} \in A \overline{\bar{\sigma}}(\overline{\bar{x}})$, with the multiplication taken in $\mathcal{R}_{\overline{\bar{x}}}$. This shows that $\overline{\bar{\sigma}}(\overline{\bar{x}})$ is Whitney $\overline{\bar{\omega}}$-convex, with Whitney constant $A$.

Thus, (G0) holds for $A, \overline{\bar{Q}}, \overline{\bar{\omega}}$, etc.

Next, we check that (G1) holds for $A, \overline{\bar{Q}}, \overline{\bar{\omega}}$, etc.

Suppose we are given $\beta, \alpha \in \overline{\mathcal{A}}^{\bar{y}}, \overline{\bar{y}} \in \overline{\bar{Q}}^{* *}$. Then, with $y=\delta_{Q} \overline{\bar{y}}$, we have $\beta, \alpha \in \overline{\mathcal{A}}^{y}, y \in Q^{* *}$ (see (1), (12)). Hence, (G1) for $A, Q, \omega, E, f, \sigma$, etc. tells us that

$$
\partial^{\beta} \bar{P}_{\alpha}^{y}(y)=\delta_{\beta \alpha}
$$

Moreover, (6) gives

$$
\partial^{\beta} \stackrel{\overline{\bar{P}}}{\overline{\bar{y}}}(\overline{\bar{y}})=\delta_{Q}^{|\beta|-|\alpha|} \partial^{\beta} \bar{P}_{\alpha}^{y}(y) .
$$

From (23) and (24) we obtain

$$
\partial^{\beta} \stackrel{\overline{\bar{P}}}{\alpha}(\overline{\bar{y}})=\delta_{\beta \alpha}
$$

which proves (G1) for $A, \overline{\bar{Q}}, \overline{\bar{\omega}}, \overline{\bar{E}}$, etc.

Next, we check that $(G 2)$ holds for $A, \overline{\bar{Q}}, \overline{\bar{\omega}}, \overline{\bar{E}}$, etc. Suppose we have $\beta \in \mathcal{M}, \alpha \in \overline{\overline{\mathcal{A}^{y}}}, \overline{\bar{y}} \in \overline{\bar{Q}}^{* *}$, with $\beta \geq \alpha$.

Taking $y=\delta_{Q} \overline{\bar{y}}$, we then have

$$
\beta \in \mathcal{M}, \alpha \in \overline{\mathcal{A}}^{y}, y \in Q^{* *}, \beta \geq \alpha,
$$

thanks to (1), (12). Hence, (G2) for $A, Q, \omega, E, f, \sigma$, etc. tells us that

$$
\left|\partial^{\beta} \bar{P}_{\alpha}^{y}(y)\right| \leq A \delta_{Q}^{|\alpha|-|\beta|} .
$$

On the other hand, (6) gives

$$
\partial^{\beta} \stackrel{\overline{\bar{P}}}{\alpha}\left(\overline{\bar{y}}(\overline{\bar{y}})=\delta_{Q}^{|\beta|-|\alpha|} \partial^{\beta} \bar{P}_{\alpha}^{y}(y),\right.
$$

as in (24), and therefore (25) implies

$$
\left|\partial^{\beta} \bar{P}_{\alpha}^{y}(y)\right| \leq A=A \delta_{\overline{\bar{Q}}}^{|\alpha|-|\beta|} \quad(\text { see }(1)) .
$$

Thus, (G2) holds for $A, \overline{\bar{Q}}, \overline{\bar{\omega}}, \overline{\bar{E}}$, etc.

Next, we check that (G3) holds for $A, \overline{\bar{Q}}, \overline{\bar{\omega}}, \overline{\bar{E}}$, etc. 
Suppose we are given $\overline{\bar{S}} \subset \overline{\bar{E}}$ with $\#(\overline{\bar{S}}) \leq k_{\text {old }}^{\#}$, together with $\overline{\bar{y}} \in \overline{\bar{Q}}{ }^{* *}$ and $\alpha \in \overline{\overline{\mathcal{A}}}^{\overline{\bar{y}}}$. Then we set $S=\delta_{Q} \overline{\bar{S}} \subset E$ (see (2), (3)), $y=\delta_{Q} \overline{\bar{y}} \in Q^{* *}$ (see (1), (5)). We have

$S \subset E \quad$ with $\quad \#(S) \leq k_{\text {old }}^{\#}, y \in Q^{* *}, \quad$ and $\quad \alpha \in \overline{\mathcal{A}}^{y}$. (See (12).)

Hence, hypothesis (G3) for $A, Q, \omega, E$, etc., produces a function $\varphi_{\alpha}^{S} \in$ $C_{\text {loc }}^{m, \omega}\left(\mathbb{R}^{n}\right)$, satisfying conditions (G3)(a),(b),(c). We define $\overline{\bar{\varphi}} \underset{\alpha}{\bar{S}}$ as in (7). We will check that (G3)(a),(b),(c) hold for $\underset{\alpha}{\bar{\varphi}}, \overline{\bar{S}}, \overline{\bar{E}}$, etc.

First, we check (G3)(a). Suppose we are given $\beta, \overline{\bar{x}}^{\prime}, \overline{\bar{x}}^{\prime \prime}$, with

$$
|\beta|=m, \quad \overline{\bar{x}}^{\prime}, \overline{\bar{x}}^{\prime \prime} \in \mathbb{R}^{n}, \quad\left|\overline{\bar{x}}^{\prime}-\overline{\bar{x}}^{\prime \prime}\right| \leq \delta_{\overline{\bar{Q}}}=1 .
$$

Then, setting $x^{\prime}=\delta_{Q} \overline{\bar{x}}^{\prime}, x^{\prime \prime}=\delta_{Q} \overline{\bar{x}}^{\prime \prime}$, we have $\left|x^{\prime}-x^{\prime \prime}\right| \leq \delta_{Q}$, and

$$
\begin{aligned}
& \left|\partial^{\beta} \underset{\bar{\varphi}}{\overline{\bar{S}}} \underset{\overline{\bar{S}}}{\overline{x^{\prime}}}\left(\overline{\bar{x}}^{\prime}\right)-\partial^{\beta} \overline{\bar{\varphi}}_{\alpha}^{\overline{\bar{S}}}\left(\overline{\bar{x}}^{\prime \prime}\right)\right|=\delta_{Q}^{|\beta|-|\alpha|}\left|\partial^{\beta} \varphi_{\alpha}^{S}\left(x^{\prime}\right)-\partial^{\beta} \varphi_{\alpha}^{S}\left(x^{\prime \prime}\right)\right| \text { (see (7)) } \\
& =\delta_{Q}^{m-|\alpha|}\left|\partial^{\beta} \varphi_{\alpha}^{S}\left(x^{\prime}\right)-\partial^{\beta} \varphi_{\alpha}^{S}\left(x^{\prime \prime}\right)\right| \\
& \leq A \delta_{Q}^{-1}\left|x^{\prime}-x^{\prime \prime}\right|+a_{0}^{\text {old }}(A) \cdot \frac{\omega\left(\left|x^{\prime}-x^{\prime \prime}\right|\right)}{\omega\left(\delta_{Q}\right)}
\end{aligned}
$$

(thanks to hypothesis (G3)(a) for $A, Q, \omega, E$, etc. and the fact that $\left|x^{\prime}-x^{\prime \prime}\right| \leq \delta_{Q}$ )

$$
\begin{aligned}
& =A\left|\overline{\bar{x}}^{\prime}-\overline{\bar{x}}^{\prime \prime}\right|+a_{0}^{\text {old }}(A) \cdot \frac{\omega\left(\delta_{Q} \cdot\left|\overline{\bar{x}}^{\prime}-\overline{\bar{x}}^{\prime \prime}\right|\right)}{\omega\left(\delta_{Q}\right)} \\
& =A\left|\overline{\bar{x}}^{\prime}-\overline{\bar{x}}^{\prime \prime}\right|+a_{0}^{\text {old }}(A) \cdot \overline{\bar{\omega}}\left(\left|\overline{\bar{x}}^{\prime}-\overline{\bar{x}}^{\prime \prime}\right|\right) \quad(\text { see }(4)) .
\end{aligned}
$$

This shows that (G3)(a) holds for $\underset{\bar{\varphi}}{\overline{\bar{S}}}, \underset{\alpha}{\bar{E}}$, etc.; and also that $\underset{\bar{\varphi}}{\overline{\bar{S}}} \underset{\alpha}{\bar{S}} \in C_{\ell o c}^{m, \overline{\bar{\omega}}}\left(\mathbb{R}^{n}\right)$.

Next, we check (G3)(b) for $\underset{\bar{\varphi}}{\overline{\bar{S}}}, \overline{\bar{S}}, \overline{\bar{E}}$, etc.

Suppose $\overline{\bar{x}} \in \overline{\bar{S}}$. Then $x=\delta_{Q} \overline{\bar{x}}$ belongs to $S$, and therefore (G3)(b) tells us that

$$
J_{x}\left(\varphi_{\alpha}^{S}\right) \in \frac{A \delta_{Q}^{|\alpha|-m}}{\omega\left(\delta_{Q}\right)} \cdot \sigma(x) .
$$

Consequently,

$$
J_{\overline{\bar{x}}}\left(\varphi_{\alpha}^{S} \circ \tau\right) \in\left\{\frac{A \delta_{Q}^{|\alpha|-m}}{\omega\left(\delta_{Q}\right)} \cdot[P \circ \tau]: P \in \sigma(x)\right\} .
$$


On the other hand (7) and (9) show that $\underset{\bar{\varphi}}{\overline{\bar{S}}}=\delta_{Q}^{-|\alpha|} \cdot\left(\varphi_{\alpha}^{S} \circ \tau\right)$, and therefore (26) gives

$$
J_{\overline{\bar{x}}}(\overline{\bar{\varphi}} \underset{\bar{\alpha}}{\bar{S}}) \in\left\{A \cdot\left(\omega\left(\delta_{Q}\right) \cdot \delta_{Q}^{m}\right)^{-1} \cdot[P \circ \tau]: P \in \sigma\left(\delta_{Q} \overline{\bar{x}}\right)\right\}
$$

Comparing this to the definition $(10)$ of $\overline{\bar{\sigma}}(\overline{\bar{x}})$, we find that

$$
J_{\overline{\bar{x}}}(\stackrel{\overline{\bar{\varphi}}}{\overline{\bar{S}}}) \in A \cdot \overline{\bar{\sigma}}(\overline{\bar{x}}) .
$$

Since $\delta_{\overline{\bar{Q}}}=1$, and hence also $\overline{\bar{\omega}}\left(\delta_{\overline{\bar{Q}}}\right)=1$ (because $\overline{\bar{\omega}}$ is a regular modulus of continuity), it follows that

$$
J_{\overline{\bar{x}}}(\overline{\bar{\varphi}} \overline{\overline{\bar{S}}}) \in \frac{A \delta_{\overline{\bar{Q}}}^{|\alpha|-m}}{\overline{\bar{\omega}}\left(\delta_{\overline{\bar{Q}}}\right)} \cdot \overline{\bar{\sigma}}(\overline{\bar{x}}) .
$$

This shows that $(\mathrm{G} 3)(\mathrm{b})$ holds for $\underset{\alpha}{\overline{\bar{\varphi}}} \underset{\overline{\bar{S}}}{\bar{E}}, \overline{\bar{E}}$, etc.

Next, we check that (G3)(c) holds for $\underset{\bar{\varphi}}{\overline{\bar{S}}} \underset{\alpha}{\bar{E}}, \underset{\bar{E}}{\bar{E}}$, etc. Hypothesis (G3)(c) for $\varphi_{\alpha}^{S}, E$, etc., tells us that $J_{y}\left(\varphi_{\alpha}^{S}\right)=\bar{P}_{\alpha}^{y}$. From the definitions (7) and (6), we see that $J_{\overline{\bar{y}}}(\stackrel{\bar{\varphi}}{\bar{\varphi}} \overline{\bar{S}})=\delta_{Q}^{-|\alpha|} J_{y}\left(\varphi_{\alpha}^{S}\right) \circ \tau$ and $\overline{\bar{P}}_{\alpha}^{\overline{\bar{y}}}=\delta_{Q}^{-|\alpha|} \bar{P}_{\alpha}^{y} \circ \tau$, with $\tau$ as in (9). (Here, we use also (5)).

Therefore,

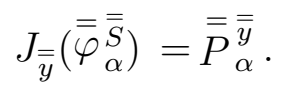

This proves $(\mathrm{G} 3)(\mathrm{c})$ for $\underset{\bar{\varphi}}{\overline{\bar{\varphi}}} \stackrel{\overline{\bar{S}}}{,}, \underset{\bar{E}}{\bar{E}}$, etc.

We have now checked (G3)(a),(b),(c) for $\underset{\bar{\varphi}}{\overline{\bar{S}}}, \underset{\bar{E}}{\bar{E}}$, etc. Thus, (G3) holds for $A, \overline{\bar{Q}}, \overline{\bar{\omega}}, \overline{\bar{E}}, \overline{\bar{f}}, \overline{\bar{\sigma}}, \overline{\overline{\mathcal{A}}}^{\overline{\bar{y}}},\left(\overline{\bar{P}}^{\overline{\bar{y}}}\right)_{\alpha \in \overline{\overline{\mathcal{A}}}}$.

Next, we check that (G4) holds for $A, \overline{\bar{Q}}, \overline{\bar{\omega}}, \overline{\bar{E}}$, etc.

Suppose $\overline{\bar{S}} \subset \overline{\bar{E}}$ with $\#(\overline{\bar{S}}) \leq k_{\text {old }}^{\#}$. We define $S=\delta_{Q} \cdot \overline{\bar{S}} \quad$ (see $\left.(2)\right)$. Thus, $S \subset E$ with $\#(S) \leq k_{\text {old }}^{\#}$. (See (3).) Applying hypothesis (G4) for $A, Q, \omega, E$, etc., we obtain a function $F^{S} \in C^{m, \omega}\left(\mathbb{R}^{n}\right)$, satisfying (G4)(a),(b),(c).

We then define $\stackrel{\overline{\bar{F}}}{\overline{\bar{S}}}$ by (11). Thus, $\stackrel{\overline{\bar{F}}}{\overline{\bar{S}}} \in C^{m, \overline{\bar{\omega}}}\left(\mathbb{R}^{n}\right)$.

We check that $(\mathrm{G} 4)(\mathrm{a}),(\mathrm{b}),(\mathrm{c})$ hold for $\stackrel{\bar{F}}{\overline{\bar{S}}}, A, \overline{\bar{Q}}, \overline{\bar{E}}$, etc.

We first check (G4)(a) for $\stackrel{\overline{\bar{F}}}{\overline{\bar{S}}}, A, \overline{\bar{Q}}$, etc. From (11), we have

$$
\left\|\partial^{\beta} \overline{\bar{F}} \overline{\overline{\bar{S}}}\right\|_{C^{0}\left(\mathbb{R}^{n}\right)}=\left(\omega\left(\delta_{Q}\right) \cdot \delta_{Q}^{m}\right)^{-1} \cdot \delta_{Q}^{|\beta|} \cdot\left\|\partial^{\beta} F^{S}\right\|_{C^{0}\left(\mathbb{R}^{n}\right)} \text { for }|\beta| \leq m .
$$


Therefore, (G4)(a) for $F^{S}, A, Q, \omega, E$, etc., implies

$$
\left\|\partial^{\beta} \stackrel{\overline{\bar{F}}}{\overline{\bar{S}}}\right\|_{C^{0}\left(\mathbb{R}^{n}\right)} \leq A \text { for }|\beta| \leq m .
$$

Since $\delta_{\overline{\bar{Q}}}=\overline{\bar{\omega}}\left(\delta_{\overline{\bar{Q}}}\right)=1$, this is equivalent to

$$
\left\|\partial^{\beta} \overline{\bar{F}}^{\overline{\bar{S}}}\right\|_{C^{0}\left(\mathbb{R}^{n}\right)} \leq A \overline{\bar{\omega}}\left(\delta_{\overline{\bar{Q}}}\right) \cdot \delta_{\overline{\bar{Q}}}^{m-|\beta|} \text { for }|\beta| \leq m .
$$

Thus, (G4)(a) holds for $\overline{\bar{F}} \overline{\bar{S}}, A, \overline{\bar{Q}}$, etc.

Next, we check $(\mathrm{G} 4)$ (b) for $\overline{\bar{F}} \overline{\bar{S}}, A, \overline{\bar{Q}}$, etc. From (11), we have, for $|\beta|=m$, that

$$
\left|\partial^{\beta} \overline{\bar{F}}^{\overline{\bar{S}}}\left(\overline{\bar{x}}^{\prime}\right)-\partial^{\beta} \overline{\bar{F}} \overline{\bar{S}}\left(\overline{\bar{x}}^{\prime \prime}\right)\right|=\left(\omega\left(\delta_{Q}\right)\right)^{-1}\left|\partial^{\beta} F^{S}\left(x^{\prime}\right)-\partial^{\beta} F^{S}\left(x^{\prime \prime}\right)\right|,
$$

with $x^{\prime}=\delta_{Q} \overline{\bar{x}}^{\prime}$ and $x^{\prime \prime}=\delta_{Q} \overline{\bar{x}^{\prime \prime}}$.

If $\left|\overline{\bar{x}}^{\prime}-\overline{\bar{x}}^{\prime \prime}\right| \leq \delta_{\overline{\bar{Q}}}$, i.e., if $\left|\overline{\bar{x}}^{\prime}-\overline{\bar{x}}^{\prime \prime}\right| \leq 1$ (see (1)), then we have $\left|x^{\prime}-x^{\prime \prime}\right| \leq \delta_{Q}$, and therefore (G4)(b) for $F^{S}, A, Q$, etc. applies. From (27) and (G4)(b) for $F^{S}, A, Q$, etc., we find that

$$
\begin{aligned}
&\left|\partial^{\beta} \overline{\bar{F}} \overline{\bar{S}}\left(\overline{\bar{x}}^{\prime}\right)-\partial^{\beta} \overline{\bar{F}} \overline{\bar{S}}\left(\overline{\bar{x}}^{\prime \prime}\right)\right| \leq A\left(\omega\left(\delta_{Q}\right)\right)^{-1} \cdot \omega\left(\left|x^{\prime}-x^{\prime \prime}\right|\right) \\
&=A \cdot \frac{\omega\left(\delta_{Q} \cdot\left|\overline{\bar{x}}^{\prime}-\overline{\bar{x}}^{\prime \prime}\right|\right)}{\omega\left(\delta_{Q}\right)}=A \cdot \overline{\bar{\omega}}\left(\left|\overline{\bar{x}}^{\prime}-\overline{\bar{x}}^{\prime \prime}\right|\right) \quad \text { (see (4)). }
\end{aligned}
$$

Thus, for $|\beta|=m, \overline{\bar{x}}^{\prime}, \overline{\bar{x}}^{\prime \prime} \in \mathbb{R}^{n}$ with $\left|\overline{\bar{x}}^{\prime}-\overline{\bar{x}}^{\prime \prime}\right| \leq \delta_{\overline{\bar{Q}}}$, we have

$$
\left|\partial^{\beta} \overline{\bar{F}} \overline{\bar{S}}\left(\overline{\bar{x}}^{\prime}\right)-\partial^{\beta} \overline{\bar{F}} \overline{\bar{S}}\left(\overline{\bar{x}}^{\prime \prime}\right)\right| \leq A \cdot \overline{\bar{\omega}}\left(\left|\overline{\bar{x}}^{\prime}-\overline{\bar{x}}^{\prime \prime}\right|\right) .
$$

This means that (G4)(b) holds for $\overline{\bar{F}} \overline{\bar{S}}, A, \overline{\bar{Q}}$, etc.

Next, we check that $(\mathrm{G} 4)$ (c) holds for $\stackrel{\overline{\bar{F}}}{\overline{\bar{S}}}, A, \overline{\bar{Q}}$, etc. Suppose $\overline{\bar{x}} \in \overline{\bar{S}}$. We set $x=\delta_{Q} \overline{\bar{x}} \in S$ (see (2)), and apply (G4)(c) for $F^{S}, A, Q$, etc. Thus,

$$
J_{x}\left(F^{S}\right) \in f(x)+A \cdot \sigma(x) .
$$

Consequently,

$$
\begin{aligned}
J_{\overline{\bar{x}}}\left(\left(\omega\left(\delta_{Q}\right) \delta_{Q}^{m}\right)^{-1} \cdot\left[F^{S} \circ \tau\right]\right) \in\left(\omega\left(\delta_{Q}\right) \delta_{Q}^{m}\right)^{-1}[f(x) \circ \tau] & \\
& +\frac{A}{\omega\left(\delta_{Q}\right) \cdot \delta_{Q}^{m}} \cdot\{P \circ \tau: P \in \sigma(x)\} .
\end{aligned}
$$

Comparing this with (8), (10), (11), we see that

$$
J_{\overline{\bar{x}}}(\overline{\bar{F}} \overline{\bar{S}}) \in \overline{\bar{f}}(\overline{\bar{x}})+A \cdot \overline{\bar{\sigma}}(\overline{\bar{x}}) .
$$

This shows that $(\mathrm{G} 4)(\mathrm{c})$ holds for $\overline{\bar{F}} \overline{\bar{S}}, A, \overline{\bar{Q}}$, etc. 
We have now checked (G4)(a), (b), (c) for $\overline{\bar{F}} \overline{\bar{S}}, A, \overline{\bar{Q}}$, etc.

Thus, (G4) holds for $A, \overline{\bar{Q}}, \overline{\bar{\omega}}, \overline{\bar{E}}$, etc.

At last, we have checked (G0), .., (G4) for $A, \overline{\bar{Q}}, \overline{\bar{\omega}}, \overline{\bar{E}}$, etc.

Thus, $A, \overline{\bar{Q}}, \overline{\bar{\omega}}, \overline{\bar{E}}, \overline{\bar{f}}, \overline{\bar{\sigma}}, \overline{\overline{\mathcal{A}}} \overline{\overline{\bar{y}}}\left(\overline{\bar{P}}_{\alpha}^{\overline{\bar{y}}}\right)_{\alpha \in \overline{\mathcal{A}}^{\overline{\bar{y}}}}$ satisfy the hypotheses of Lemma 8.1, with $\overline{\bar{Q}}$ having diameter 1 .

If Lemma 8.1 holds for cubes of diameter 1 , then we obtain for $A, \overline{\bar{Q}}, \overline{\bar{\omega}}$, etc., a function $\overline{\bar{F}} \in C^{m, \bar{\omega}}\left(\mathbb{R}^{n}\right)$, satisfying (G5), (G6), (G7) for $A, \overline{\bar{Q}}, \overline{\bar{\omega}}$, etc. Since $\delta_{\overline{\bar{Q}}}=\overline{\bar{\omega}}\left(\delta_{\overline{\bar{Q}}}\right)=1$, this means that

$$
\begin{array}{r}
\left\|\partial^{\beta} \overline{\bar{F}}\right\|_{C^{0}\left(\mathbb{R}^{n}\right)} \leq A^{\prime} \quad \text { for }|\beta| \leq m ; \\
\left|\partial^{\beta} \overline{\bar{F}}\left(\overline{x^{\prime}}\right)-\partial^{\beta} \overline{\bar{F}}\left(\overline{\overline{x^{\prime \prime}}}\right)\right| \leq A^{\prime} \cdot \overline{\bar{\omega}}\left(\left|\overline{\bar{x}}^{\prime}-\overline{\bar{x}}^{\prime \prime}\right|\right)
\end{array}
$$

for $|\beta|=m, \overline{\bar{x}}^{\prime}, \overline{\bar{x}}^{\prime \prime} \in \mathbb{R}^{n},\left|\overline{\bar{x}}^{\prime}-\overline{\bar{x}}^{\prime \prime}\right| \leq 1$; and

$$
J_{\overline{\bar{x}}}(\overline{\bar{F}}) \in \overline{\bar{f}}(\overline{\bar{x}})+A^{\prime} \cdot \overline{\bar{\sigma}}(\overline{\bar{x}}) \text { for all } \overline{\bar{x}} \in \overline{\bar{E}} \cap \overline{\bar{Q}}^{*}
$$

Here, $A^{\prime}$ is determined by $A, m, n$.

We now define $F$ on $\mathbb{R}^{n}$, by setting

$$
\begin{aligned}
F & =\left(\omega\left(\delta_{Q}\right) \cdot \delta_{Q}^{m}\right) \cdot \overline{\bar{F}} \circ \tau^{-1}, \quad \text { i.e. } \\
F(x) & =\left(\omega\left(\delta_{Q}\right) \cdot \delta_{Q}^{m}\right) \cdot \overline{\bar{F}}\left(\delta_{Q}^{-1} x\right) .
\end{aligned}
$$

Since $\overline{\bar{F}} \in C^{m, \overline{\bar{\omega}}}\left(\mathbb{R}^{n}\right)$, we have $F \in C^{m, \omega}\left(\mathbb{R}^{n}\right)$.

We will check that the function $F$ satisfies conclusions (G5), (G6), (G7) for $A, Q, \omega, E, f, \sigma$, etc., with the same constant $A^{\prime}$ as in (28), (29), (30).

First, we check (G5) for $F, A, Q, \omega$, etc. Immediately from (28), (32), we have

$$
\left\|\partial^{\beta} F\right\|_{C^{0}\left(\mathbb{R}^{n}\right)}=\left(\omega\left(\delta_{Q}\right) \cdot \delta_{Q}^{m}\right) \cdot \delta_{Q}^{-|\beta|}\left\|\partial^{\beta} \stackrel{\bar{F}}{F}\right\|_{C^{0}\left(\mathbb{R}^{n}\right)} \leq A^{\prime} \omega\left(\delta_{Q}\right) \delta_{Q}^{m-|\beta|}
$$

for $|\beta| \leq m$. Thus, (G5) holds for $F, A, Q, \omega$, etc.

Next, we check (G6) for $F, A, Q, \omega$, etc.

Suppose $|\beta|=m, x^{\prime}, x^{\prime \prime} \in \mathbb{R}^{n},\left|x^{\prime}-x^{\prime \prime}\right| \leq \delta_{Q}$. Setting $\overline{\bar{x}}^{\prime}=\delta_{Q}^{-1} x^{\prime}$, $\overline{\bar{x}}^{\prime \prime}=\delta_{Q}^{-1} x^{\prime \prime}$, we have $\left|\overline{\bar{x}}^{\prime}-\overline{\bar{x}}^{\prime \prime}\right| \leq 1$, hence (29) applies. From (29), (32) we 
obtain

$$
\begin{aligned}
\left|\partial^{\beta} F\left(x^{\prime}\right)-\partial^{\beta} F\left(x^{\prime \prime}\right)\right| & =\left(\omega\left(\delta_{Q}\right) \delta_{Q}^{m}\right) \delta_{Q}^{-|\beta|}\left|\partial^{\beta} \overline{\bar{F}}\left(\overline{\bar{x}}^{\prime}\right)-\partial^{\beta} \overline{\bar{F}}\left(\overline{\bar{x}}^{\prime \prime}\right)\right| \\
& \leq A^{\prime} \omega\left(\delta_{Q}\right) \cdot \overline{\bar{\omega}}\left(\left|\overline{\bar{x}}^{\prime}-\overline{\bar{x}}^{\prime \prime}\right|\right) \quad(\text { recall, }|\beta|=m) \\
& =A^{\prime} \omega\left(\delta_{Q}\right) \cdot \frac{\omega\left(\delta_{Q}\left|\overline{\bar{x}}^{\prime}-\overline{\bar{x}}^{\prime \prime}\right|\right)}{\omega\left(\delta_{Q}\right)} \quad(\text { see }(4)) \\
& =A^{\prime} \omega\left(\delta_{Q}\left|\overline{\bar{x}}^{\prime}-\overline{\bar{x}}^{\prime \prime}\right|\right)=A^{\prime} \omega\left(\left|x^{\prime}-x^{\prime \prime}\right|\right) .
\end{aligned}
$$

Thus, (G6) holds for $F, A, Q, \omega$, etc.

Next, we check that (G7) holds for $F, A, Q, \omega$, etc. Suppose $x \in E \cap Q^{*}$. Setting $\overline{\bar{x}}=\delta_{Q}^{-1} x$, we have $\overline{\bar{x}} \in \overline{\bar{E}} \cap \overline{\bar{Q}}^{*}$, hence (30) applies. Thus,

$$
J_{\overline{\bar{x}}}(\overline{\bar{F}}) \in \overline{\bar{f}}(\overline{\bar{x}})+A^{\prime} \cdot \overline{\bar{\sigma}}(\overline{\bar{x}}) .
$$

Consequently,

$$
\begin{aligned}
J_{x}\left(\omega\left(\delta_{Q}\right) \cdot \delta_{Q}^{m} \cdot\left[\overline{\bar{F}} \circ \tau^{-1}\right]\right) & \in \omega\left(\delta_{Q}\right) \delta_{Q}^{m} \cdot\left[(\overline{\bar{f}}(\overline{\bar{x}})) \circ \tau^{-1}\right] \\
+ & A^{\prime} \cdot\left\{\omega\left(\delta_{Q}\right) \delta_{Q}^{m} \cdot\left[\overline{\bar{P}} \circ \tau^{-1}\right]: \overline{\bar{P}} \in \overline{\bar{\sigma}}(\overline{\bar{x}})\right\}
\end{aligned}
$$

We have from (8) that

$$
\begin{aligned}
\omega\left(\delta_{Q}\right) \delta_{Q}^{m} \cdot\left[(\overline{\bar{f}}(\overline{\bar{x}})) \circ \tau^{-1}\right] & =\omega\left(\delta_{Q}\right) \cdot \delta_{Q}^{m} \cdot\left[\left(\omega\left(\delta_{Q}\right) \delta_{Q}^{m}\right)^{-1} \cdot\left\{\left(f\left(\delta_{Q} \overline{\bar{x}}\right)\right) \circ \tau\right\} \circ \tau^{-1}\right] \\
& =f\left(\delta_{Q} \overline{\bar{x}}\right)=f(x) .
\end{aligned}
$$

Together with (31), this yields

$$
J_{x}(F) \in f(x)+A^{\prime} \cdot\left\{\omega\left(\delta_{Q}\right) \delta_{Q}^{m} \cdot\left[\overline{\bar{P}} \circ \tau^{-1}\right]: \overline{\bar{P}} \in \overline{\bar{\sigma}}(\overline{\bar{x}})\right\}
$$

From (10), we see that

$$
\begin{aligned}
\left\{\omega\left(\delta_{Q}\right) \delta_{Q}^{m}\right. & \left.\cdot\left[\overline{\bar{P}} \circ \tau^{-1}\right]: \overline{\bar{P}} \in \overline{\bar{\sigma}}(\overline{\bar{x}})\right\}= \\
& =\left\{\omega\left(\delta_{Q}\right) \delta_{Q}^{m} \cdot\left[\left(\omega\left(\delta_{Q}\right) \delta_{Q}^{m}\right)^{-1}[P \circ \tau] \circ \tau^{-1}\right]: P \in \sigma\left(\delta_{Q} \overline{\bar{x}}\right)\right\} \\
& =\sigma\left(\delta_{Q} \overline{\bar{x}}\right)=\sigma(x) .
\end{aligned}
$$

Hence, (34) shows that

$$
J_{x}(F) \in f(x)+A^{\prime} \cdot \sigma(x) .
$$

Thus, (G7) holds for $F, A, Q, \omega$, etc. 
We have now shown that Lemma 8.1 holds, provided it holds in the case $\delta_{Q}=1$. For the rest of the proof of Lemma 8.1, we suppose that $\delta_{Q}=1$. We take $k_{\text {old }}^{\#}$ to be a constant determined by $m$ and $n$, satisfying

$$
k_{\text {old }}^{\#} \geq 1
$$

and

(36) $k_{\text {old }}^{\#} \geq k^{\#}$, with $k^{\#}$ as in the Strong Main Lemma for any $\overline{\mathcal{A}}<\mathcal{A}$.

(Note that (36) makes sense, since one of the hypotheses of Lemma 8.1 is that the Strong Main Lemma holds for each $\overline{\mathcal{A}}<\mathcal{A}$.)

We will take $a_{0}^{\text {old }}(A)$ to be a small enough constant, depending only on $A, m, n$, to be picked below.

Now suppose $A, Q, \omega, E, f, \sigma, \overline{\mathcal{A}}^{y}\left(y \in Q^{* *}\right)$, and $\bar{P}_{\alpha}^{y}\left(\alpha \in \overline{\mathcal{A}}^{y}, y \in Q^{* *}\right)$ are as in the hypotheses of Lemma 8.1, with $\delta_{Q}=1$, and with $k_{\text {old }}^{\#}$ and $a_{0}^{\text {old }}(A)$ as described above.

We must show that there exists $F \in C^{m, \omega}\left(\mathbb{R}^{n}\right)$, satisfying (G5), (G6), (G7).

The first step is to correct $f$, as follows.

Given $\underline{x} \in E$, we let $S=\{\underline{x}\}$. Thus, $S \subset E$ and $\#(S) \leq k_{\text {old }}^{\#}$, by (35).

Applying (G4), we obtain a function $F^{S} \in C^{m, \omega}\left(\mathbb{R}^{n}\right)$, satisfying in particular $\left|\partial^{\beta} F^{S}(\underline{x})\right| \leq A$ for $|\beta| \leq m$, and $J_{\underline{x}}\left(F^{S}\right) \in f(\underline{x})+A \sigma(\underline{x})$.

Setting $\tilde{f}(\underline{x})=J_{\underline{x}}\left(F^{S}\right)$, we have

$$
\begin{aligned}
\left|\partial^{\beta}[\tilde{f}(\underline{x})](\underline{x})\right| & \leq A \text { for }|\beta| \leq m, \text { and } \\
\tilde{f}(\underline{x})-f(\underline{x}) & \in A \sigma(\underline{x}), \text { for each } \underline{x} \in E .
\end{aligned}
$$

(In $(37)$, note that since $\tilde{f}(\underline{x}) \in \mathcal{R}_{\underline{x}}$, the expression $\partial^{\beta}[\tilde{f}(\underline{x})](\underline{x})$ makes sense; it means $\left(\frac{\partial}{\partial y}\right)^{\beta}[\tilde{f}(\underline{x})](y)$ evaluated at $y=\underline{x}$.)

In view of (37) and (G4), we have the following property of $\tilde{f}$.

(38) Given $S \subset E$ with $\#(S) \leq k_{\text {old }}^{\#}$, there exists $F^{S} \in C^{m, \omega}\left(\mathbb{R}^{n}\right)$, with

(a) $\left\|\partial^{\beta} F^{S}\right\|_{C^{0}\left(\mathbb{R}^{n}\right)} \leq A$ for $|\beta| \leq m$;

(b) $\left|\partial^{\beta} F^{S}\left(x^{\prime}\right)-\partial^{\beta} F^{S}\left(x^{\prime \prime}\right)\right| \leq A \cdot \omega\left(\left|x^{\prime}-x^{\prime \prime}\right|\right)$ for $|\beta|=m, x^{\prime}, x^{\prime \prime} \in \mathbb{R}^{n}$, $\left|x^{\prime}-x^{\prime \prime}\right| \leq 1$

(c) $J_{x}\left(F^{S}\right) \in \tilde{f}(x)+2 A \cdot \sigma(x)$ for all $x \in S$.

(Recall that $\delta_{Q}=1$, hence also $\omega\left(\delta_{Q}\right)=1$ since $\omega$ is a regular modulus of continuity.)

We now check the following. 
(39) Claim: For each $y \in Q^{* *}$, the hypotheses of the Strong Main Lemma for $\overline{\mathcal{A}}^{y}$ are satisfied, with our present

$$
2 A, a_{0}^{\text {old }}(A), \omega, E, y,\left(\bar{P}^{y}\right)_{\alpha \in \overline{\mathcal{A}} y}, \tilde{f}, \sigma,
$$

in place of

$$
C, \bar{a}_{0}, \omega, E, y^{0},\left(P_{\alpha}\right)_{\alpha \in \overline{\mathcal{A}}}, f, \sigma
$$

in the statement of the Strong Main Lemma for $\overline{\mathcal{A}}^{y}$.

In fact, $2 A$ and $a_{0}^{\text {old }}(A)$ are positive constants; $\omega$ is a regular modulus of continuity and $E \subset \mathbb{R}^{n}$ is finite (by hypothesis of Lemma 8.1); $y \in \mathbb{R}^{n}$; $\bar{P}_{\alpha}^{y} \in \mathcal{P}$ is indexed by $\alpha \in \overline{\mathcal{A}}^{y}$ (again, by hypothesis of Lemma 8.1); and, for each $x \in E$, we have $\tilde{f}(x) \in \mathcal{R}_{x}$ and $\sigma(x) \subset \mathcal{R}_{x}$ (yet again by hypothesis of Lemma 8.1). To check (39), we must show that conditions (SL0),..,(SL5) hold for $2 A, a_{0}^{\text {old }}(A), \omega, E$, etc.

Condition (SL0) for $2 A, a_{0}^{\text {old }}(A)$, etc., says that, for each $x \in E$, the set $\sigma(x)$ is Whitney $\omega$-convex $x$, with Whitney constant $2 A$. This follows at once from our present hypothesis (G0).

Condition (SL1) for $2 A, a_{0}^{\text {old }}(A)$, etc., says that $\partial^{\beta} \bar{P}_{\alpha}^{y}(y)=\delta_{\beta \alpha}$ for $\beta, \alpha \in \overline{\mathcal{A}}^{y}$. Since $y \in Q^{* *}$, this is immediate from our present hypothesis (G1).

Condition (SL2) for $2 A, a_{0}^{\text {old }}(A)$, etc., says that $\left|\partial^{\beta} \bar{P}_{\alpha}^{y}(y)\right| \leq 2 A$ for $\beta \in$ $\mathcal{M}, \alpha \in \overline{\mathcal{A}}^{y}$ with $\beta \geq \alpha$.

Since $\delta_{Q}=\omega\left(\delta_{Q}\right)=1$ and $y \in Q^{* *}$, this follows at once from our present hypothesis (G2).

Condition (SL3) for $2 A, a_{0}^{\text {old }}(A)$, etc., says the following.

(40) Given $\alpha \in \overline{\mathcal{A}}^{y}$ and $S \subset E$ with $\#(S) \leq k^{\#}$, there exists $\varphi_{\alpha}^{S} \in C_{\ell o c}^{m, \omega}\left(\mathbb{R}^{n}\right)$, with

(a) $\left|\partial^{\beta} \varphi_{\alpha}^{S}\left(x^{\prime}\right)-\partial^{\beta} \varphi_{\alpha}^{S}\left(x^{\prime \prime}\right)\right| \leq 2 A\left|x^{\prime}-x^{\prime \prime}\right|+a_{0}^{\text {old }}(A) \cdot \omega\left(\left|x^{\prime}-x^{\prime \prime}\right|\right)$ for $|\beta|=m, x^{\prime}, x^{\prime \prime} \in \mathbb{R}^{n},\left|x^{\prime}-x^{\prime \prime}\right| \leq 1$

(b) $J_{x}\left(\varphi_{\alpha}^{S}\right) \in 2 A \sigma(x)$ for all $x \in S$; and

(c) $J_{y}\left(\varphi_{\alpha}^{S}\right)=\bar{P}_{\alpha}^{y}$.

Here $k^{\#}$ is as in the Strong Main Lemma for $\overline{\mathcal{A}}^{y}$.

We recall from the hypotheses of Lemma 8.1 that $\overline{\mathcal{A}}^{y}<\mathcal{A}$, since $y \in Q^{* *}$. Hence, (36) gives $k_{\text {old }}^{\#} \geq k^{\#}$. Also, we again recall that $\delta_{Q}=\omega\left(\delta_{Q}\right)=1$. In view of the above remarks, (40) follows at once from our present hypothesis (G3). Thus, (SL3) holds for $2 A, a_{0}^{\text {old }}(A)$, etc.

Condition (SL4) for $2 A, a_{0}^{\text {old }}(A)$, etc., says the following. 
(41) Given $S \subset E$ with $\#(S) \leq k^{\#}$, there exists $F^{S} \in C^{m, \omega}\left(\mathbb{R}^{n}\right)$, with

(a) $\left\|F^{S}\right\|_{C^{m, \omega}\left(\mathbb{R}^{n}\right)} \leq 2 A$; and

(b) $J_{x}\left(F^{S}\right) \in \tilde{f}(x)+2 A \sigma(x)$ for all $x \in S$.

Here again, $k^{\#}$ is as in the Strong Main Lemma for $\overline{\mathcal{A}}^{y}$, hence $k^{\#} \leq k_{\text {old }}^{\#}$, by (36). Consequently, (41) follows at once from (38).

(Here, we use the precise definition of the $C^{m, \omega}$-norm from the section on Notation and Preliminaries.) Thus, (SL4) holds for $2 A, a_{0}^{\text {old }}(A)$, etc.

Condition (SL5) for $2 A, a_{0}^{\text {old }}(A)$, etc., says that

(42) $a_{0}^{\text {old }}(A)$ is less than a small enough constant determined by $2 A, m, n$.

We now specify $a_{0}^{\text {old }}(A)$, which so far was "to be picked later". We simply pick $a_{0}^{\text {old }}(A)$ to be a positive number, determined by $A, m, n$, and small enough to satisfy (42). Thus, (SL5) holds for $2 A, a_{0}^{\text {old }}(A)$, etc.

The verification of our claim (39) is complete.

We now recall two hypotheses of Lemma 8.1: For $y \in Q^{* *}$, we have $\overline{\mathcal{A}}^{y}<\mathcal{A}$; and the Strong Main Lemma holds for $\overline{\mathcal{A}}<\mathcal{A}$.

Consequently, from (39), we may draw the following conclusion.

(43) Given $y \in Q^{* *}$, there exists $F \in C^{m, \omega}\left(\mathbb{R}^{n}\right)$, with $\|F\|_{C^{m, \omega}\left(\mathbb{R}^{n}\right)} \leq A^{\prime}$, and $J_{x}(F) \in \tilde{f}(x)+A^{\prime} \sigma(x)$ for all $x \in E \cap B\left(y, a^{\prime}\right)$.

Here, $A^{\prime}$ and $a^{\prime}$ depend only on $A, m, n$. We fix $A^{\prime}$ and $a^{\prime}$ for the rest of our proof of Lemma 8.1.

We write $A_{1}, A_{2}, \cdots$ for constants depending only on $A, m, n$.

To exploit (43), we use a partition of unity

$$
1=\sum_{\nu=1}^{\nu_{\max }} \theta_{\nu} \quad \text { on } Q^{*} ;
$$

with

$$
\begin{aligned}
\left\|\theta_{\nu}\right\|_{C^{m+1}\left(\mathbb{R}^{n}\right)} & \leq A_{1} ; \\
\operatorname{supp} \theta_{\nu} & \subset B\left(y_{\nu}, a^{\prime}\right) \quad \text { with } y_{\nu} \in Q^{* *} ; \text { and } \\
\nu_{\max } & \leq A_{2} .
\end{aligned}
$$

We can find $\left\{\theta_{\nu}\right\}$ as in $(44), \ldots,(47)$, since $\delta_{Q}=1$.

Since each $y_{\nu}$ belongs to $Q^{* *}$, we may apply (43). 
Thus, for each $\nu\left(1 \leq \nu \leq \nu_{\max }\right)$, we obtain $F_{\nu} \in C^{m, \omega}\left(\mathbb{R}^{n}\right)$, with

$$
\left\|F_{\nu}\right\|_{C^{m, \omega}\left(\mathbb{R}^{n}\right)} \leq A^{\prime}
$$

and

$$
J_{x}\left(F_{\nu}\right) \in \tilde{f}(x)+A^{\prime} \cdot \sigma(x) \text { for all } x \in E \cap B\left(y_{\nu}, a^{\prime}\right) .
$$

In particular, we have

$$
J_{x}\left(F_{\nu}\right)=\tilde{f}(x)+A^{\prime} \cdot P_{\nu}^{x} \quad \text { for all } x \in E \cap B\left(y_{\nu}, a^{\prime}\right),
$$

with

$$
P_{\nu}^{x} \in \sigma(x), \quad \text { for all } x \in E \cap B\left(y_{\nu}, a^{\prime}\right) .
$$

From (48), we have $\left|\partial^{\beta} F_{\nu}(x)\right| \leq A^{\prime}$ for $|\beta| \leq m, x \in \mathbb{R}^{n}$.

From (37), we have $\left|\partial^{\beta}(\tilde{f}(x))(x)\right| \leq A$ for $|\beta| \leq m, x \in E$.

Together with (49), these estimates show that

$$
\left|\partial^{\beta} P_{\nu}^{x}(x)\right| \leq A_{3} \quad \text { for }|\beta| \leq m, x \in E \cap B\left(y_{\nu}, a^{\prime}\right) .
$$

If we hadn't taken the trouble to pass from $f(x)$ to $\tilde{f}(x)$ as above, then we would not have been able to obtain (51).

We now define

$$
F=\sum_{\nu=1}^{\nu_{\max }} \theta_{\nu} \cdot F_{\nu} \in C^{m, \omega}\left(\mathbb{R}^{n}\right) .
$$

From (45), (47), (48), we conclude that

$$
\|F\|_{C^{m, \omega}\left(\mathbb{R}^{n}\right)} \leq A_{4} .
$$

Next, fix $x \in Q^{*} \cap E$ and let $\Omega=\left\{\nu: 1 \leq \nu \leq \nu_{\max }\right.$ and $\left.x \in B\left(y_{\nu}, a^{\prime}\right)\right\}$.

Then, with "." denoting multiplication in $\mathcal{R}_{x}$, we may argue as follows. We have

$$
\text { (54) } \begin{aligned}
J_{x}(F) & =\sum_{1 \leq \nu \leq \nu_{\max }} J_{x}\left(\theta_{\nu}\right) \cdot J_{x}\left(F_{\nu}\right) \\
& =\sum_{\nu \in \Omega} J_{x}\left(\theta_{\nu}\right) \cdot J_{x}\left(F_{\nu}\right) \\
& =\sum_{\nu \in \Omega} J_{x}\left(\theta_{\nu}\right) \cdot \tilde{f}(x)+\sum_{\nu \in \Omega} J_{x}\left(\theta_{\nu}\right) \cdot A^{\prime} P_{\nu}^{x} \\
& =\sum_{1 \leq \nu \leq \nu_{\max }} J_{x}\left(\theta_{\nu}\right) \cdot \tilde{f}(x)+\sum_{\nu \in \Omega} J_{x}\left(\theta_{\nu}\right) \cdot A^{\prime} P_{\nu}^{x} \\
& =\tilde{f}(x)+A^{\prime} \cdot \sum_{\nu \in \Omega} J_{x}\left(\theta_{\nu}\right) \cdot P_{\nu}^{x} .
\end{aligned}
$$


We note that

$$
\left|\partial^{\beta} \frac{J_{x}\left(\theta_{\nu}\right)}{A_{5}}(x)\right| \leq 1 \quad \text { for }|\beta| \leq m, \quad \text { thanks to }(45),
$$

and also

$$
\left|\partial^{\beta}\left(\frac{P_{\nu}^{x}}{A_{6}}\right)(x)\right| \leq 1 \quad \text { for }|\beta| \leq m, \nu \in \Omega, \quad \text { thanks to }(51) .
$$

We may take $A_{6} \geq 1$ in (56). Hence, we have also

$$
\left(\frac{P_{\nu}^{x}}{A_{6}}\right) \in \sigma(x) \quad \text { for } \nu \in \Omega, \quad \text { thanks to }(50) \text {. }
$$

(Recall that $\sigma(x)$ is convex and symmetric about 0 .)

From (55), (56), (57), and from hypothesis (G0) of Lemma 8.1, we conclude that

$$
J_{x}\left(\theta_{\nu}\right) \cdot P_{\nu}^{x} \in A_{7} \sigma(x) \text { for all } \nu \in \Omega \text {. }
$$

(Here, we take $\delta=1$ in the definition of Whitney $\omega$-convexity.)

From (47), (58), and the fact that $\sigma(x)$ is convex and symmetric about 0 , we conclude that

$$
\sum_{\nu \in \Omega} J_{x}\left(\theta_{\nu}\right) \cdot P_{\nu}^{x} \in A_{8} \sigma(x),
$$

and consequently (54) implies that

$$
J_{x}(F) \in \tilde{f}(x)+A_{9} \sigma(x) .
$$

From (37) we recall that $f(x)-\tilde{f}(x) \in A \sigma(x)$. Hence, (59) yields

$$
J_{x}(F) \in f(x)+A_{10} \sigma(x) .
$$

Thus, we have shown that

$$
J_{x}(F) \in f(x)+A_{10} \sigma(x) \text { for all } x \in E \cap Q^{*} .
$$

In particular, we have produced a function $F \in C^{m, \omega}\left(\mathbb{R}^{n}\right)$, satisfying (53) and (60). Since the constants $A_{4}$ and $A_{10}$ in (53) and (60) are determined by $A, m, n$, we see that $F$ satisfies the conclusions (G5), (G6), (G7), and that the constant called $A^{\prime}$ in (G5), (G6), (G7) may be taken to depend only on $A, m, n$.

Thus, we have proven Lemma 8.1 in the case of $\delta_{Q}=1$. Since we already reduced the general case to the case $\delta_{Q}=1$, the proof of Lemma 8.1 is complete. 


\section{Set-Up for the Main Induction}

In this section, we give the set-up for the proof of Lemma 5.2 in the monotonic case. We fix $m, n \geq 1$, and $\mathcal{A} \subseteq \mathcal{M}$.

We let $k^{\#}$ be a large enough integer, determined by $m$ and $n$, to be picked later. We suppose we are given the following data:

- Constants $C_{0}, a_{1}, a_{2}>0$.

- A regular modulus of continuity $\omega$.

- A finite set $E \subset \mathbb{R}^{n}$.

- For each $x \in E$, an $m$-jet $f(x) \in \mathcal{R}_{x}$ and a set $\sigma(x) \subset \mathcal{R}_{x}$.

- A point $y^{0} \in \mathbb{R}^{n}$.

- A family of polynomials $P_{\alpha} \in \mathcal{P}$, indexed by $\alpha \in \mathcal{A}$.

We fix $C_{0}, a_{1}, a_{2}, \omega, E, f, \sigma, y^{0},\left(P_{\alpha}\right)_{\alpha \in \mathcal{A}}$ until the end of Section 16. We make the following assumptions.

(SU0) $\mathcal{A}$ is monotonic, and $\mathcal{A} \neq \mathcal{M}$.

(SU1) The Strong Main Lemma holds for all $\overline{\mathcal{A}}<\mathcal{A}$.

(SU2) For each $x \in E$, the set $\sigma(x) \subset \mathcal{R}_{x}$ is Whitney $\omega$-convex at $x$, with Whitney constant $C_{0}$.

(SU3) $\partial^{\beta} P_{\alpha}\left(y^{0}\right)=\delta_{\beta \alpha}$ for all $\beta, \alpha \in \mathcal{A}$.

(SU4) $\left|\partial^{\beta} P_{\alpha}\left(y^{0}\right)-\delta_{\beta \alpha}\right| \leq a_{1}$ for all $\beta \in \mathcal{M}, \alpha \in \mathcal{A}$.

(SU5) $a_{1}$ is less than a small enough constant determined by $C_{0}, m$ and $n$.

(SU6) Given $\alpha \in \mathcal{A}$ and $S \subset E$ with $\#(S) \leq k^{\#}$, there exists $\varphi_{\alpha}^{S} \in C_{\ell o c}^{m, \omega}\left(\mathbb{R}^{n}\right)$, with

(a) $\left|\partial^{\beta} \varphi_{\alpha}^{S}\left(x^{\prime}\right)-\partial^{\beta} \varphi_{\alpha}^{S}\left(x^{\prime \prime}\right)\right| \leq a_{2} \cdot \omega\left(\left|x^{\prime}-x^{\prime \prime}\right|\right)$ for all $\beta, x, x^{\prime}$, with $|\beta|=m$, $x^{\prime}, x^{\prime \prime} \in \mathbb{R}^{n},\left|x^{\prime}-x^{\prime \prime}\right| \leq 1$;

(b) $J_{x}\left(\varphi_{\alpha}^{S}\right) \in C_{0} \sigma(x)$ for all $x \in S$; and

(c) $J_{y^{0}}\left(\varphi_{\alpha}^{S}\right)=P_{\alpha}$.

(SU7) $a_{2}$ is less than a small enough constant determined by $a_{1}, C_{0}, m$, and $n$.

(SU8) Given $S \subset E$ with $\#(S) \leq k^{\#}$, there exists $F^{S} \in C^{m, \omega}\left(\mathbb{R}^{n}\right)$, with

(a) $\left\|F^{S}\right\|_{C^{m, \omega}\left(\mathbb{R}^{n}\right)} \leq C_{0}$; and

(b) $J_{x}\left(F^{S}\right) \in f(x)+C_{0} \sigma(x)$ for all $x \in S$. 
The main effort of this paper goes into proving the following result.

Lemma 9.1 Assume (SU0),..,(SU8). Then there exists $F \in C^{m, \omega}\left(\mathbb{R}^{n}\right)$ with

(a) $\|F\|_{C^{m, \omega}\left(\mathbb{R}^{n}\right)} \leq A$, and

(b) $J_{x}(F) \in f(x)+A \sigma(x)$ for all $x \in E \cap B\left(y^{0}, a\right)$;

here, $A$ and $a$ are determined by $a_{1}, a_{2}, m, n, C_{0}$.

In this section, we prove the following result.

Lemma 9.2 Lemma 9.1 implies Lemma 5.2.

Proof: Assume that Lemma 9.1 holds. To establish Lemma 5.2, we fix $\mathcal{A} \subset \mathcal{M}$, with $\mathcal{A} \neq \mathcal{M}$, and we assume that the Strong Main Lemma holds for all $\overline{\mathcal{A}}<\mathcal{A}$. We must prove the Weak Main Lemma for $\mathcal{A}$ under the above assumptions. We may assume also that $\mathcal{A}$ is monotonic, thanks to Lemma 7.1.

Let $C, a_{0}, \omega, E, f, \sigma, y^{0},\left(P_{\alpha}\right)_{\alpha \in \mathcal{A}}$ be as in the hypotheses of the Weak Main Lemma for $\mathcal{A}$, with the "small enough constant" in (WL5) to be picked below. We take $k^{\#}$ as in (SU0),..., (SU8).

We then pick the constants $C_{0}, a_{1}, a_{2}$ as follows.

First, we take $C_{0}=C$.

Next, we pick $a_{1}>0$, depending only on $C, m$ and $n$, and small enough to satisfy (SU5).

Finally, we pick $a_{2}>0$, depending only on $C, m, n$, and small enough to satisfy (SU7). (This can be done, since our $a_{1}$ depends only on $C, m$ and $n$.)

We now take the "small enough constant determined by $C, m, n$ " in (WL5) to be small enough that (WL5) implies $a_{0}<\min \left(a_{1}, a_{2}\right)$.

With the constants picked as explained above, we have satisfied (SU5) and (SU7), and we have ensured that

$$
a_{0}<\min \left(a_{1}, a_{2}\right),
$$

since we are assuming (WL5).

We now check that $C_{0}, a_{1}, a_{2}, \omega, E, f, \sigma, y^{0},\left(P_{\alpha}\right)_{\alpha \in \mathcal{A}}$ satisfy conditions (SU0),..., (SU8).

In fact, we have assumed (SU0) and (SU1), and we have picked the constants $a_{1}, a_{2}$ to satisfy (SU5) and (SU7).

Conditions (SU2), (SU3), (SU4) are immediate from hypotheses (WL0), (WL1), (WL2), since $C=C_{0}$ and $a_{0}<a_{1}$.

Similarly, conditions (SU6) and (SU8) are immediate from hypotheses (WL3), (WL4), since $C=C_{0}$ and $a_{0}<a_{2}$.

Thus, as claimed, $C_{0}, a_{1}, a_{2}, \omega, E, f, \sigma, y^{0},\left(P_{\alpha}\right)_{\alpha \in \mathcal{A}}$ satisfy (SU0), .., (SU8). 
Since we are assuming Lemma 9.1, it follows that there exists $F \in$ $C^{m, \omega}\left(\mathbb{R}^{n}\right)$, with

$$
\begin{aligned}
& \|F\|_{C^{m, \omega}\left(\mathbb{R}^{n}\right)} \leq A, \text { and with } \\
& J_{x}(F) \in f(x)+A \sigma(x), \text { for all } x \in E \cap B\left(y^{0}, a\right),
\end{aligned}
$$

where $A$ and $a$ are determined by $C_{0}, m, n, a_{1}, a_{2}$. However, we picked $C_{0}, a_{1}, a_{2}$ above, so that $C_{0}=C$, and $a_{1}$ and $a_{2}$ are determined by $C, m, n$.

Consequently, $A$ and $a$ in (1) are also determined by $C, m, n$. Hence, (1) is equivalent to the conclusions (WL6), (WL7) of the Weak Main Lemma for $\mathcal{A}$.

Thus, we have proven that Lemma 9.1, together with the Strong Main Lemma for all $\overline{\mathcal{A}}<\mathcal{A}$, implies the Weak Main Lemma for $\mathcal{A}$. This shows that Lemma 9.1 implies Lemma 5.2. The proof of Lemma 9.2 is complete.

We begin the work of proving Lemma 9.1. Until the end of Section 16, we fix $C_{0}, a_{1}, a_{2}, \omega, E, f, \sigma, y^{0},\left(P_{\alpha}\right)_{\alpha \in \mathcal{A}}$, and we assume that (SU0), ., (SU8) are satisfied. Also, until the end of Section 16, except in Section 15, we write $c, C, C^{\prime}$, etc., to denote constants determined by $C_{0}, m, n$ in (SU0), . , (SU8); and we call such constants "controlled". However, in Section 15, c, C, $C^{\prime}$, etc. will denote constants depending only on $m$ and $n$.

Also, until the end of Section 16, we fix a constant $k_{\text {old }}^{\#}$, depending only on $m$ and $n$, as in Lemma 8.1.

\section{Applying Helly's Theorem on Convex Sets}

In this section, we start the proof of Lemma 9.1 by applying repeatedly the following well-known result (Helly's theorem; see [18]).

Lemma 10.0 Let $\mathcal{F}$ be a family of compact, convex subsets of $\mathbb{R}^{d}$. Suppose that any $(d+1)$ of the sets in $\mathcal{F}$ have non-empty intersection. Then the whole family $\mathcal{F}$ has non-empty intersection.

We assume $(\mathrm{SU} 0, \ldots, 8)$, and adopt the conventions of Section 9.

For $M>0, S \subset E, y \in \mathbb{R}^{n}$, we define

$$
\begin{array}{r}
\mathcal{K}_{f}(y, S, M)=\left\{J_{y}(F): F \in C^{m, \omega}\left(\mathbb{R}^{n}\right),\|F\|_{C^{m, \omega}\left(\mathbb{R}^{n}\right)} \leq M,\right. \\
\left.J_{x}(F) \in f(x)+M \sigma(x) \text { on } S\right\} .
\end{array}
$$

For $M>0, k \geq 1, y \in \mathbb{R}^{n}$, we then define

$$
\mathcal{K}_{f}(y, k, M)=\bigcap\left\{\mathcal{K}_{f}(y, S, M): S \subset E, \#(S) \leq k\right\} .
$$

Note that $\mathcal{K}_{f}(y, S, M)$ is a convex subset of $\mathcal{P}$. 
Moreover, if $F_{i} \in C^{m, \omega}\left(\mathbb{R}^{n}\right)$ with $\left\|F_{i}\right\|_{C^{m, \omega}\left(\mathbb{R}^{n}\right)} \leq M$ and $J_{x}\left(F_{i}\right) \in f(x)+$ $M \sigma(x)$ for all $x \in S$, for $i=1, \ldots$, then by Ascoli's theorem, we may pick out a subsequence of $\left\{F_{i}\right\}$ that converges in $C^{m}$-norm on compact sets in $\mathbb{R}^{n}$. The limit $F$ of that subsequence will satisfy $F \in C^{m, \omega}\left(\mathbb{R}^{n}\right),\|F\|_{C^{m, \omega}\left(\mathbb{R}^{n}\right)} \leq$ $M$, and $J_{x}(F) \in f(x)+M \sigma(x)$ for all $x \in S$. (Here, we recall that $\sigma(x)$ is closed, since it is Whitney $\omega$-convex.) Consequently, $\mathcal{K}_{f}(y, S, M)$ is a compact, convex subset of $\mathcal{P}$. Hence, also, $\mathcal{K}_{f}(y, k, M)$ is compact and convex.

Lemma 10.1 Suppose we are given $k_{1}^{\#}$, with $k^{\#} \geq(D+1) \cdot k_{1}^{\#}$, and $k_{1}^{\#} \geq 1$. Let $C_{0}$ be as in (SU0) ,.., (SU8). Then $\mathcal{K}_{f}\left(y, k_{1}^{\#}, C_{0}\right)$ is non-empty, for each $y \in \mathbb{R}^{n}$.

Proof: Fix $y \in \mathbb{R}^{n}$, and let $S_{1}, \ldots, S_{D+1} \subseteq E$, with $\#\left(S_{i}\right) \leq k_{1}^{\#}$ for each $i$.

Let $S=S_{1} \cup \cdots \cup S_{D+1}$. Thus, $S \subset E$ with $\#(S) \leq k^{\#}$. Applying (SU8) to $S$, and setting $P=J_{y}\left(F^{S}\right)$ with $F^{S}$ as in (SU8), we have $P \in \mathcal{K}_{f}\left(y, S_{i}, C_{0}\right)$ for $i=1, \ldots, D+1$. Consequently, any $(D+1)$ of the sets $\mathcal{K}_{f}\left(y, S, C_{0}\right)$ $\left(S \subset E, \#(S) \leq k_{1}^{\#}\right)$ have non-empty intersection. Hence, Lemma 10.1 follows from Helly's theorem and (2).

Lemma 10.2 Suppose $k_{1}^{\#} \geq(D+1) \cdot k_{2}^{\#}$, let $A>0$, and suppose we are given $P \in \mathcal{K}_{f}\left(y, k_{1}^{\#}, A\right)$. Then, for $\left|y^{\prime}-y\right| \leq 1$, there exists $P^{\prime} \in$ $\mathcal{K}_{f}\left(y^{\prime}, k_{2}^{\#}, A\right)$, with

$\left|\partial^{\beta}\left(P-P^{\prime}\right)(y)\right|,\left|\partial^{\beta}\left(P-P^{\prime}\right)\left(y^{\prime}\right)\right| \leq C A \omega\left(\left|y-y^{\prime}\right|\right) \cdot\left|y-y^{\prime}\right|^{m-|\beta|}$ for $|\beta| \leq m$.

Proof: For $S \subset E$, define

$$
\begin{aligned}
\mathcal{K}_{\text {temp }}(S)=\left\{J_{y^{\prime}}(F):\right. & F \in C^{m, \omega}\left(\mathbb{R}^{n}\right),\|F\|_{C^{m, \omega}\left(\mathbb{R}^{n}\right)} \leq A, \\
& \left.J_{x}(F) \in f(x)+A \sigma(x) \text { on } S, J_{y}(F)=P\right\} .
\end{aligned}
$$

Each $\mathcal{K}_{\text {temp }}(S)$ is a compact convex subset of $\mathcal{P}$, as we see from Ascoli's theorem, just as above for $\mathcal{K}_{f}(y, S, M)$. Let $S_{1}, \ldots, S_{D+1} \subset E$, with \# $\left(S_{i}\right) \leq$ $k_{2}^{\#}$ for each $i$. Set $S=S_{1} \cup \cdots \cup S_{D+1}$; note that $S \subset E$, with $\#(S) \leq k_{1}^{\#}$. Since $P \in \mathcal{K}_{f}\left(y, k_{1}^{\#}, A\right)$, there exists $F \in C^{m, \omega}\left(\mathbb{R}^{n}\right)$ with $\|F\|_{C^{m, \omega}\left(\mathbb{R}^{n}\right)} \leq A$, $J_{x}(F) \in f(x)+A \sigma(x)$ on $S$, and $J_{y}(F)=P$. In particular, $J_{y^{\prime}}(F)$ belongs to $\mathcal{K}_{\text {temp }}\left(S_{i}\right)$ for each $i$. Thus, any $(D+1)$ of the sets $\mathcal{K}_{\text {temp }}(S)(S \subset E, \#(S) \leq$ $k_{2}^{\#}$ ) have non-empty intersection. By Helly's theorem, the intersection of all the sets $\mathcal{K}_{\text {temp }}(S)\left(S \subset E, \#(S) \leq k_{2}^{\#}\right)$ is non-empty. Let $P^{\prime}$ belong to this intersection. Thus, by definition, $P^{\prime}$ has the following property.

(3) Given $S \subset E$ with $\#(S) \leq k_{2}^{\#}$, there exists $F \in C^{m, \omega}\left(\mathbb{R}^{n}\right)$, with $\|F\|_{C^{m, \omega}\left(\mathbb{R}^{n}\right)} \leq A, J_{x}(F) \in f(x)+A \sigma(x)$ for all $x \in S, J_{y}(F)=P$, $J_{y^{\prime}}(F)=P^{\prime}$. 
In particular, we have $P^{\prime} \in \mathcal{K}_{f}\left(y, k_{2}^{\#}, A\right)$. Also, taking $F$ as in (3) with $S=$ empty set, we have from Taylor's theorem that

$$
\begin{aligned}
\left|\partial^{\beta}\left(P-P^{\prime}\right)\left(y^{\prime}\right)\right| & =\left|\sum_{|\gamma| \leq m-|\beta|} \frac{1}{\gamma !}\left(\partial^{\gamma+\beta} P(y)\right) \cdot\left(y^{\prime}-y\right)^{\gamma}-\partial^{\beta} P^{\prime}\left(y^{\prime}\right)\right| \\
& =\left|\sum_{|\gamma| \leq m-|\beta|} \frac{1}{\gamma !}\left(\partial^{\gamma+\beta} F(y)\right) \cdot\left(y^{\prime}-y\right)^{\gamma}-\partial^{\beta} F\left(y^{\prime}\right)\right| \\
& \leq C A \omega\left(\left|y-y^{\prime}\right|\right) \cdot\left|y-y^{\prime}\right|^{m-|\beta|}
\end{aligned}
$$

and similarly for $\left|\partial^{\beta}\left(P-P^{\prime}\right)\left(y^{\prime}\right)\right|$. The proof of Lemma 10.2 is complete.

Lemma 10.3 Suppose $k^{\#} \geq(D+1) \cdot k_{1}^{\#}$, and let $y \in B\left(y^{0}, a_{1}\right)$ be given.

Then there exist polynomials $P_{\alpha}^{y} \in \mathcal{P}$, indexed by $\alpha \in \mathcal{A}$, with the following properties:

$(\mathrm{WL} 1)^{y} \partial^{\beta} P_{\alpha}^{y}(y)=\delta_{\beta \alpha}$ for $\beta, \alpha \in \mathcal{A}$.

$(\mathrm{WL} 2)^{y}\left|\partial^{\beta} P_{\alpha}^{y}(y)-\delta_{\beta \alpha}\right| \leq C a_{1}$ for all $\alpha \in \mathcal{A}, \beta \in \mathcal{M}$.

$(\mathrm{WL} 3)^{y}$ Given $\alpha \in \mathcal{A}$ and $S \subset E$ with $\#(S) \leq k_{1}^{\#}$, there exists $\varphi_{\alpha}^{S} \in C_{\text {loc }}^{m, \omega}\left(\mathbb{R}^{n}\right)$, with

(a) $\left|\partial^{\beta} \varphi_{\alpha}^{S}\left(x^{\prime}\right)-\partial^{\beta} \varphi_{\alpha}^{S}\left(x^{\prime \prime}\right)\right| \leq C a_{2} \omega\left(\left|x^{\prime}-x^{\prime \prime}\right|\right)$ for $|\beta|=m, x^{\prime}, x^{\prime \prime} \in \mathbb{R}^{n}$, $\left|x^{\prime}-x^{\prime \prime}\right| \leq 1$

(b) $J_{x}\left(\varphi_{\alpha}^{S}\right) \in C \sigma(x)$ for all $x \in S$; and

(c) $J_{y}\left(\varphi_{\alpha}^{S}\right)=P_{\alpha}^{y}$

Proof: We may assume $y \neq y^{0}$, since otherwise the lemma is immediate from $(\mathrm{SU} 3,4,6)$.

For $\alpha \in \mathcal{A}, S \subset E$, we define

$$
\begin{array}{r}
\mathcal{K}_{\alpha}(S)=\left\{J_{y}(\varphi): \varphi \in C_{\ell o c}^{m, \omega}\left(\mathbb{R}^{n}\right), J_{y^{0}}(\varphi)=P_{\alpha}, J_{x}(\varphi) \in C_{0} \sigma(x) \text { for all } x \in S\right. \\
\text { and }\left|\partial^{\beta} \varphi\left(x^{\prime}\right)-\partial^{\beta} \varphi\left(x^{\prime \prime}\right)\right| \leq a_{2} \omega\left(\left|x^{\prime}-x^{\prime \prime}\right|\right) \text { for }|\beta|=m, \\
\left.x^{\prime}, x^{\prime \prime} \in \mathbb{R}^{n},\left|x^{\prime}-x^{\prime \prime}\right| \leq 1\right\}
\end{array}
$$

(Here, $a_{2}$ and $C_{0}$ are as in $(\mathrm{SU} 0, \ldots, 8)$.)

Each $\mathcal{K}_{\alpha}(S)$ is a convex subset of $\mathcal{P}$. We check that $\mathcal{K}_{\alpha}(S)$ is also compact. In fact, suppose $P_{i} \in \mathcal{K}_{\alpha}(S)$ for $i=1,2, \ldots$.

Then there exist $\varphi_{i} \in C_{\ell o c}^{m, \omega}\left(\mathbb{R}^{n}\right)$, with $J_{y}\left(\varphi_{i}\right)=P_{i}, J_{y^{0}}\left(\varphi_{i}\right)=P_{\alpha}$, $J_{x}\left(\varphi_{i}\right) \in C_{0} \sigma(x)$ for all $x \in S$, and $\left|\partial^{\beta} \varphi_{i}\left(x^{\prime}\right)-\partial^{\beta} \varphi_{i}\left(x^{\prime \prime}\right)\right| \leq a_{2} \omega\left(\left|x^{\prime}-x^{\prime \prime}\right|\right)$ for $|\beta|=m, x^{\prime}, x^{\prime \prime} \in \mathbb{R}^{n},\left|x^{\prime}-x^{\prime \prime}\right| \leq 1$. 
In particular, since $J_{y^{0}}\left(\varphi_{i}\right)$ is fixed and the $\partial^{\beta} \varphi_{i}(|\beta|=m)$ have a common modulus of continuity, Ascoli's theorem picks out a subsequence $\left\{\varphi_{i_{\nu}}\right\}$ that converges in $C^{m}$ norm on compact subsets of $\mathbb{R}^{n}$. The limit $\varphi$ will then satisfy $\varphi \in C_{\ell o c}^{m, \omega}\left(\mathbb{R}^{n}\right), J_{y^{0}}(\varphi)=P_{\alpha}, J_{x}(\varphi) \in C_{0} \sigma(x)$ for all $x \in S$, and $\left|\partial^{\beta} \varphi\left(x^{\prime}\right)-\partial^{\beta} \varphi\left(x^{\prime \prime}\right)\right| \leq a_{2} \omega\left(\left|x^{\prime}-x^{\prime \prime}\right|\right)$ for $|\beta|=m, x^{\prime}, x^{\prime \prime} \in \mathbb{R}^{n},\left|x^{\prime}-x^{\prime \prime}\right| \leq 1$.

(Here, we use the fact that $\sigma(x)$ is closed, since it is Whitney $\omega$-convex.)

It follows that $J_{y}(\varphi)$ belongs to $\mathcal{K}_{\alpha}(S)$.

On the other hand, since $\varphi_{i_{\nu}} \rightarrow \varphi$ in $C^{m}$-norm on compact sets, we have $P_{i_{\nu}}=J_{y}\left(\varphi_{i_{\nu}}\right) \rightarrow J_{y}(\varphi)$ in $\mathcal{P}$. Thus, any sequence $\left\{P_{i}\right\}$ of points of $\mathcal{K}_{\alpha}(S)$ has a subsequence that converges to a point of $\mathcal{K}_{\alpha}(S)$. Hence, as claimed, $\mathcal{K}_{\alpha}(S)$ is compact.

Next, suppose $S_{1}, \ldots, S_{D+1} \subset E$, with $\#\left(S_{i}\right) \leq k_{1}^{\#}$ for each $i$. Set $S=S_{1} \cup \cdots \cup S_{D+1}$; note that $S \subset E$ with $\#(S) \leq k^{\#}$.

Applying (SU6), and letting $\varphi_{\alpha}^{S}$ be as in (SU6), we see that $J_{y}\left(\varphi_{\alpha}^{S}\right)$ belongs to $\mathcal{K}_{\alpha}\left(S_{i}\right)$ for each $i$. Thus, any $(D+1)$ of the sets $\mathcal{K}_{\alpha}(S)(S \subset$ $E, \#(S) \leq k_{1}^{\#}$ ) have non-empty intersection.

Consequently, by Helly's theorem, there exists $\bar{P}_{\alpha}^{y} \in \mathcal{P}$, belonging to each $\mathcal{K}_{\alpha}(S)\left(S \subset E, \#(S) \leq k_{1}^{\#}\right)$.

By definition, the $\bar{P}_{\alpha}^{y}$ have the following property.

(4) Given $S \subset E$ with $\#(S) \leq k_{1}^{\#}$, and given $\alpha \in \mathcal{A}$, there exists $\bar{\varphi}_{\alpha}^{S} \in$ $C_{\text {loc }}^{m, \omega}\left(\mathbb{R}^{n}\right)$, with

(a) $\left|\partial^{\beta} \bar{\varphi}_{\alpha}^{S}\left(x^{\prime}\right)-\partial^{\beta} \bar{\varphi}_{\alpha}^{S}\left(x^{\prime \prime}\right)\right| \leq a_{2} \omega\left(\left|x^{\prime}-x^{\prime \prime}\right|\right)$ for $|\beta|=m, x^{\prime}, x^{\prime \prime} \in \mathbb{R}^{n}$, $\left|x^{\prime}-x^{\prime \prime}\right| \leq 1$

(b) $J_{x}\left(\bar{\varphi}_{\alpha}^{S}\right) \in C_{0} \sigma(x)$ for all $x \in S$;

(c) $J_{y^{0}}\left(\bar{\varphi}_{\alpha}^{S}\right)=P_{\alpha}$;

(d) $J_{y}\left(\bar{\varphi}_{\alpha}^{S}\right)=\bar{P}_{\alpha}^{y}$.

In particular, taking $S=$ empty set in (4), we find that, for $|\beta| \leq m$, we have

$$
\begin{aligned}
\mid \partial^{\beta} \bar{P}_{\alpha}^{y}(y) & -\sum_{|\gamma| \leq m-|\beta|} \frac{1}{\gamma !}\left(\partial^{\gamma+\beta} P_{\alpha}\left(y^{0}\right)\right) \cdot\left(y-y^{0}\right)^{\gamma} \mid \\
& =\left|\partial^{\beta} \bar{\varphi}_{\alpha}^{S}(y)-\sum_{|\gamma| \leq m-|\beta|} \frac{1}{\gamma !}\left(\partial^{\gamma+\beta} \bar{\varphi}_{\alpha}^{S}\left(y^{0}\right)\right) \cdot\left(y-y^{0}\right)^{\gamma}\right| \\
& \leq C a_{2} \omega\left(\left|y-y^{0}\right|\right) \cdot\left|y-y^{0}\right|^{m-|\beta|} \\
& \leq C a_{2} .
\end{aligned}
$$

(Recall that $\omega$ is a regular modulus of continuity and that $y \in B\left(y^{0}, a_{1}\right)$, with $a_{1}<1$ by (SU5).) 
From (SU4,5), we have $\left|\partial^{\beta^{\prime}} P_{\alpha}\left(y^{0}\right)\right| \leq 2$ for all $\alpha \in \mathcal{A}, \beta^{\prime} \in \mathcal{M}$.

Since also $y \in B\left(y^{0}, a_{1}\right)$ with $a_{2}<a_{1}<1$ (see (SU5,7)), we have $\left|\partial^{\gamma+\beta} P_{\alpha}\left(y^{0}\right)\right| \cdot\left|\left(y-y^{0}\right)^{\gamma}\right| \leq C a_{1}$ for $\gamma \neq 0$, and therefore (5) implies

$$
\left|\partial^{\beta} \bar{P}_{\alpha}^{y}(y)-\partial^{\beta} P_{\alpha}\left(y^{0}\right)\right| \leq C a_{1} \text { for } \alpha \in \mathcal{A}, \beta \in \mathcal{M} .
$$

In view of (SU4), we therefore have

$$
\left|\partial^{\beta} \bar{P}_{\alpha}^{y}(y)-\delta_{\beta \alpha}\right| \leq C a_{1} \quad \text { for } \alpha \in \mathcal{A}, \beta \in \mathcal{M} .
$$

From (6) and (SU5), we see that the matrix $\left(\partial^{\beta} \bar{P}_{\alpha}^{y}(y)\right)_{\beta, \alpha \in \mathcal{A}}$ is invertible, and its inverse matrix $\left(M_{\alpha^{\prime} \alpha}\right)_{\alpha^{\prime}, \alpha \in \mathcal{A}}$ satisfies

$$
\left|M_{\alpha^{\prime} \alpha}-\delta_{\alpha^{\prime} \alpha}\right| \leq C a_{1} \quad \text { for } \alpha^{\prime}, \alpha \in \mathcal{A} .
$$

For each $\alpha \in \mathcal{A}$, we now define

$$
P_{\alpha}^{y}=\sum_{\alpha^{\prime} \in \mathcal{A}} \bar{P}_{\alpha^{\prime}}^{y} M_{\alpha^{\prime} \alpha} \in \mathcal{P} .
$$

By definition of $M_{\alpha^{\prime} \alpha}$, we have

$$
\partial^{\beta} P_{\alpha}^{y}(y)=\sum_{\alpha^{\prime} \in \mathcal{A}}\left(\partial^{\beta} \bar{P}_{\alpha^{\prime}}^{y}(y)\right) M_{\alpha^{\prime} \alpha}=\delta_{\beta \alpha} \quad \text { for } \beta, \alpha \in \mathcal{A} .
$$

Also, from (6), (7), (8), we see that

$$
\left|\partial^{\beta} P_{\alpha}^{y}(y)-\delta_{\beta \alpha}\right| \leq C a_{1} \quad \text { for all } \beta \in \mathcal{M}, \alpha \in \mathcal{A} .
$$

Next, suppose we are given $S \subset E$, with $\#(S) \leq k_{1}^{\#}$. For each $\alpha \in \mathcal{A}$, let $\bar{\varphi}_{\alpha}^{S} \in C_{\ell o c}^{m, \omega}\left(\mathbb{R}^{n}\right)$ be as in (4), and then define

$$
\varphi_{\alpha}^{S}=\sum_{\alpha^{\prime} \in \mathcal{A}} \bar{\varphi}_{\alpha^{\prime}}^{S} M_{\alpha^{\prime} \alpha} \quad \text { for each } \alpha \in \mathcal{A}
$$

Thus, $\varphi_{\alpha}^{S} \in C_{\ell o c}^{m, \omega}\left(\mathbb{R}^{n}\right)$. From (4)(a), (7), (SU5), and (11), we see that

$$
\left|\partial^{\beta} \varphi_{\alpha}^{S}\left(x^{\prime}\right)-\partial^{\beta} \varphi_{\alpha}^{S}\left(x^{\prime \prime}\right)\right| \leq C a_{2} \omega\left(\left|x^{\prime}-x^{\prime \prime}\right|\right)
$$

for $|\beta|=m, x^{\prime}, x^{\prime \prime} \in \mathbb{R}^{n},\left|x^{\prime}-x^{\prime \prime}\right| \leq 1$.

From (4)(b), (7), (SU5), and (11), we see that

$$
J_{x}\left(\varphi_{\alpha}^{S}\right) \in C \sigma(x) \text { for all } x \in S .
$$

(Here, we also use (SU2).)

From $(4)(d),(8),(11)$, we obtain

$$
J_{y}\left(\varphi_{\alpha}^{S}\right)=P_{\alpha}^{y} \quad \text { for } \alpha \in \mathcal{A} .
$$


Thus, we have proven the following.

(12) Given $\alpha \in \mathcal{A}$ and $S \subset E$ with $\#(S) \leq k_{1}^{\#}$, there exists $\varphi_{\alpha}^{S} \in C_{\ell o c}^{m, \omega}(\mathbb{R})$, with

(a) $\left|\partial^{\beta} \varphi_{\alpha}^{S}\left(x^{\prime}\right)-\partial^{\beta} \varphi_{\alpha}^{S}\left(x^{\prime \prime}\right)\right| \leq C a_{2} \omega\left(\left|x^{\prime}-x^{\prime \prime}\right|\right)$ for $|\beta|=m, x^{\prime}, x^{\prime \prime} \in \mathbb{R}^{n}$, $\left|x^{\prime}-x^{\prime \prime}\right| \leq 1$

(b) $J_{x}\left(\varphi_{\alpha}^{S}\right) \in C \sigma(x)$ for all $x \in S$; and

(c) $J_{y}\left(\varphi_{\alpha}^{S}\right)=P_{\alpha}^{y}$.

The conclusions of Lemma 10.3 are (9), (10) and (12).

The proof of Lemma 10.3 is complete.

Lemma 10.4 Suppose $k^{\#} \geq k_{1}^{\#} \cdot(D+1)$ and $k_{1}^{\#} \geq k_{2}^{\#} \cdot(D+1)$.

Let $y \in B\left(y^{0}, a_{1}\right)$, and let $\left(P_{\alpha}^{y}\right)_{\alpha \in \mathcal{A}}$ satisfy conclusions $(\mathrm{WL} 1)^{y}$, (WL2)y , $(\mathrm{WL} 3)^{y}$, as in the conclusion of Lemma 10.3. Let $y^{\prime} \in \mathbb{R}^{n}$ be given .

Then there exist polynomials $\tilde{P}_{\alpha}^{y^{\prime}}(\alpha \in \mathcal{A})$, with the following property: with

Given $\alpha \in \mathcal{A}$ and $S \subset E$ with $\#(S) \leq k_{2}^{\#}$, there exists $\varphi_{\alpha}^{S} \in C_{\ell o c}^{m, \omega}\left(\mathbb{R}^{n}\right)$,

(a) $\left|\partial^{\beta} \varphi_{\alpha}^{S}\left(x^{\prime}\right)-\partial^{\beta} \varphi_{\alpha}^{S}\left(x^{\prime \prime}\right)\right| \leq C a_{2} \omega\left(\left|x^{\prime}-x^{\prime \prime}\right|\right) \quad$ for $|\beta|=m, x^{\prime}, x^{\prime \prime} \in \mathbb{R}^{n}$, $\left|x^{\prime}-x^{\prime \prime}\right| \leq 1$

(b) $J_{x}\left(\varphi_{\alpha}^{S}\right) \in C \sigma(x)$ for all $x \in S$;

(c) $J_{y}\left(\varphi_{\alpha}^{S}\right)=P_{\alpha}^{y} ;$ and

(d) $J_{y^{\prime}}\left(\varphi_{\alpha}^{S}\right)=\tilde{P}_{\alpha}^{y^{\prime}}$.

Proof: The lemma is trivial for $y^{\prime}=y$; we just set $\tilde{P}_{\alpha}^{y^{\prime}}=P_{\alpha}$ and apply $(\text { WL3 } 3)^{y}$.

Suppose $y^{\prime} \neq y$. For $\alpha \in \mathcal{A}, S \subset E$, we set

$$
\begin{array}{r}
\mathcal{K}^{[\alpha]}(S)=\left\{J_{y^{\prime}}(\varphi): \varphi \in C_{\ell o c}^{m, \omega}\left(\mathbb{R}^{n}\right) ;\left|\partial^{\beta} \varphi\left(x^{\prime}\right)-\partial^{\beta} \varphi\left(x^{\prime \prime}\right)\right| \leq C a_{2} \omega\left(\left|x^{\prime}-x^{\prime \prime}\right|\right)\right. \\
\text { for }|\beta|=m, x^{\prime}, x^{\prime \prime} \in \mathbb{R}^{n},\left|x^{\prime}-x^{\prime \prime}\right| \leq 1 ; \\
\left.J_{x}\left(\varphi_{\alpha}^{S}\right) \in C \sigma(x) \text { for all } x \in S ; \quad J_{y}(\varphi)=P_{\alpha}^{y}\right\},
\end{array}
$$

with $C$ as in $(\mathrm{WL} 1)^{y},(\mathrm{WL} 2)^{y},(\mathrm{WL} 3)^{y}$.

As in the proof of Lemma 10.3, we see that $\mathcal{K}^{[\alpha]}(S)$ is a compact, convex subset of $\mathcal{P}$. Suppose $S_{1}, \ldots, S_{D+1} \subset E$, with $\#\left(S_{1}\right) \leq k_{2}^{\#}$ for each $i$.

Set $S=S_{1} \cup \cdots \cup S_{D+1}$; note that $S \subset E$, with $\#(S) \leq k_{1}^{\#}$.

Taking $\varphi_{\alpha}^{S} \in C_{\ell o c}^{m, \omega}\left(\mathbb{R}^{n}\right)$ as in $(\mathrm{WL} 3)^{y}$, we see that $J_{y^{\prime}}\left(\varphi_{\alpha}^{S}\right)$ belongs to $\mathcal{K}^{[\alpha]}\left(S_{i}\right)$ for each $i$.

Thus, $\mathcal{K}^{[\alpha]}\left(S_{1}\right) \cap \cdots \cap \mathcal{K}^{[\alpha]}\left(S_{D+1}\right)$ is non-empty. 
Applying Helly's theorem, we see that, for each $\alpha \in \mathcal{A}$, there exists $\tilde{P}_{\alpha}^{y^{\prime}} \in \mathcal{P}$, belonging to $\mathcal{K}^{[\alpha]}(S)$ for each $S \subset E$ with $\#(S) \leq k_{2}^{\#}$.

Properties (a),.., (d) for $\tilde{P}_{\alpha}^{y^{\prime}}$ now follow from the definition of $\mathcal{K}^{[\alpha]}(S)$. The proof of Lemma 10.4 is complete.

Next, for $y \in \mathbb{R}^{n}, k \geq 1, M>0$, we define

$$
\mathcal{K}_{f}^{\#}(y, k, M)=\left\{P \in \mathcal{K}_{f}(y, k, M): \partial^{\beta} P(y)=0 \text { for all } \beta \in \mathcal{A}\right\} .
$$

Lemma 10.5 Suppose $k^{\#} \geq(D+1) \cdot k_{1}^{\#}$ and $k_{1}^{\#} \geq 1$ Then, for a large enough controlled constant $C$, the set $\mathcal{K}_{f}^{\#}\left(y, k_{1}^{\#}, C\right)$ is non-empty for each $y \in B\left(y^{0}, a_{1}\right)$.

Proof: Fix $y \in B\left(y^{0}, a_{1}\right)$. By Lemma 10.1, there exists $P \in \mathcal{K}_{f}\left(y, k_{1}^{\#}, C_{0}\right)$. Thus, $P \in \mathcal{P}$, and

(13) given $S \subset E$ with $\#(S) \leq k_{1}^{\#}$, there exists $F^{S} \in C_{\text {loc }}^{m, \omega}\left(\mathbb{R}^{n}\right)$, with

(a) $\left\|F^{S}\right\|_{C^{m, \omega}\left(\mathbb{R}^{n}\right)} \leq C_{0}$;

(b) $J_{x}\left(F^{S}\right) \in f(x)+C_{0} \sigma(x)$ for all $x \in S$; and

(c) $J_{y}\left(F^{S}\right)=P$.

By Lemma 10.3, there exist $P_{\alpha}^{y} \in \mathcal{P}($ all $\alpha \in \mathcal{A})$, with properties (WL1) ${ }^{y}$, $(\mathrm{WL} 2)^{y},(\mathrm{WL} 3)^{y}$. We define

$$
\tilde{P}=P-\sum_{\alpha \in \mathcal{A}}\left(\partial^{\alpha} P(y)\right) \cdot P_{\alpha}^{y} \in \mathcal{P}
$$

For $\beta \in \mathcal{A}$, we have

$$
\partial^{\beta} \tilde{P}(y)=\partial^{\beta} P(y)-\sum_{\alpha \in \mathcal{A}}\left(\partial^{\alpha} P(y)\right) \cdot\left(\partial^{\beta} P_{\alpha}^{y}(y)\right)=0
$$

thanks to $(\mathrm{WL} 1)^{y}$.

Taking $S=$ empty set in (13), we see that

$$
\left|\partial^{\beta} P(y)\right| \leq C \quad \text { for all } \beta \in \mathcal{M} .
$$

We introduce a cutoff function $\theta$ on $\mathbb{R}^{n}$, with

$$
\|\theta\|_{C^{m+1}\left(\mathbb{R}^{n}\right)} \leq C, \theta=1 \quad \text { on } B(y, 1 / 20), \text { supp } \theta \subset B(y, 1 / 10) \text {. }
$$

Now, let $S \subset E$, with $\#(S) \leq k^{\#}$. We let $F^{S}$ be as in (13), and for each $\alpha \in \mathcal{A}$, we let $\varphi_{\alpha}^{S}$ be as in (WL3) ${ }^{y}$. 
We then define

$$
\tilde{F}^{S}=F^{S}-\sum_{\alpha \in \mathcal{A}}\left[\partial^{\alpha} P(y)\right] \cdot \theta \varphi_{\alpha}^{S} \in C^{m, \omega}\left(\mathbb{R}^{n}\right)
$$

(Note that $F^{S} \in C^{m, \omega}\left(\mathbb{R}^{n}\right), \varphi_{\alpha}^{S} \in C_{\text {loc }}^{m, \omega}\left(\mathbb{R}^{n}\right), \theta \in C^{m+1}\left(\mathbb{R}^{n}\right)$, and $\operatorname{supp} \theta \subset$ $B(y, 1 / 10)$. Hence, $\tilde{F}^{S} \in C^{m, \omega}\left(\mathbb{R}^{n}\right)$, as asserted in (18).)

Let us estimate the derivatives of $\tilde{F}^{S}$. From (WL2)y and (WL3)y (a), (c), we have

$$
\left|\partial^{\beta} \varphi_{\alpha}^{S}\left(x^{\prime}\right)\right| \leq C \quad \text { for }|\beta| \leq m \text { and } x^{\prime} \in B(y, 1)
$$

(Recall that $a_{1}<1$, by (SU5).)

From (19), (WL3)(a), and (17), we see that

$$
\left\|\theta \varphi_{\alpha}^{S}\right\|_{C^{m, \omega}\left(\mathbb{R}^{n}\right)} \leq C \quad \text { for } \alpha \in \mathcal{A} .
$$

From (13)(a), (16), (18), (20), we conclude that

$$
\left\|\tilde{F}^{S}\right\|_{C^{m, \omega}\left(\mathbb{R}^{n}\right)} \leq C .
$$

Next, suppose $x \in S \cap B(y, 1)$. Then (WL3)(b) and (19) show that

$$
J_{x}\left(c \varphi_{\alpha}^{S}\right) \in \sigma(x), \quad \text { and } \quad\left|\partial^{\beta}\left[J_{x}\left(c \varphi_{\alpha}^{S}\right)\right](x)\right| \leq 1 \quad \text { for }|\beta| \leq m .
$$

Also, (17) gives

$$
\left|\partial^{\beta}\left[J_{x}(c \theta)\right](x)\right| \leq 1 \quad \text { for }|\beta| \leq m
$$

Recalling our assumption (SU2), and taking $\delta=1$ in the definition of Whitney $\omega$-convexity, we see that

$$
J_{x}\left(\theta \varphi_{\alpha}^{S}\right) \in C \sigma(x) \text { for } x \in S \cap B(y, 1) .
$$

On the other hand, if $x \in S \backslash B(y, 1)$, then from (17) we see that $J_{x}\left(\theta \varphi_{\alpha}^{S}\right)=0 \in C \sigma(x)$.

Thus, we have proven that

$$
J_{x}\left(\theta \varphi_{\alpha}^{S}\right) \in C \sigma(x) \text { for all } x \in S, \alpha \in \mathcal{A} \text {. }
$$

Hence, from (13)(b), (16), (18), (22), we obtain

$$
J_{x}\left(\tilde{F}^{S}\right) \in f(x)+C \sigma(x) \text { for all } x \in S .
$$


Next, note that (13)(c), (14), (WL3)(c), (17), (18) show that

$$
\begin{aligned}
J_{y}\left(\tilde{F}^{S}\right) & =J_{y}\left(F^{S}\right)-\sum_{\alpha \in \mathcal{A}}\left[\partial^{\alpha} P(y)\right] \cdot J_{y}\left(\theta \varphi_{\alpha}^{S}\right) \\
& =J_{y}\left(F^{S}\right)-\sum_{\alpha \in \mathcal{A}}\left[\partial^{\alpha} P(y)\right] \cdot J_{y}\left(\varphi_{\alpha}^{S}\right) \\
& =P-\sum_{\alpha \in \mathcal{A}}\left[\partial^{\alpha} P(y)\right] \cdot P_{\alpha}^{y} \\
& =\tilde{P} .
\end{aligned}
$$

In view of (21), (23), (24), we have proven the following result.

Given $S \subset E$ with $\#(S) \leq k_{1}^{\#}$, there exists $\tilde{F}^{S} \in C^{m, \omega}\left(\mathbb{R}^{n}\right)$, with $\left\|\tilde{F}^{S}\right\|_{C^{m, \omega}\left(\mathbb{R}^{n}\right)} \leq C, J_{x}\left(\tilde{F}^{S}\right) \in f(x)+C \sigma(x)$ for all $x \in S$, and $J_{y}\left(\tilde{F}^{S}\right)=\tilde{P}$.

By definition, this means that $\tilde{P} \in \mathcal{K}_{f}\left(y, k_{1}^{\#}, C\right)$.

This, in turn, implies $\tilde{P} \in \mathcal{K}_{f}^{\#}\left(y, k_{1}^{\#}, C\right)$, thanks to (15).

Thus, $\mathcal{K}_{f}^{\#}\left(y, k_{k}^{\#}, C\right)$ is non-empty.

The proof of Lemma 10.5 is complete.

\section{A Calderón-Zygmund Decomposition}

In this section, we again place ourselves in the setting of Section 9, and we assume (SU0, .,8). We fix a cube $Q^{0} \subset \mathbb{R}^{n}$, with the following properties.

$$
\begin{aligned}
& Q^{0} \text { is centered at } y^{0} . \\
& \left(Q^{0}\right)^{* * *} \subset B\left(y^{0}, a_{1}\right) . \\
& c a_{1}<\delta_{Q^{0}}<a_{1} .
\end{aligned}
$$

Recall that a subcube $Q \subseteq Q^{0}$ is called "dyadic" if $Q=Q^{0}$ or else $Q$ arises from $Q^{0}$ by successive "bisection". A dyadic cube $Q \neq Q^{0}$ arises by bisecting its dyadic "parent" $Q^{+}$, which is again a dyadic cube, with $\delta_{Q^{+}}=2 \delta_{Q}$. Only $Q^{0}$ and subcubes of $Q^{0}$ may be called "dyadic", according to the above definition.

Two distinct dyadic cubes will be said to "abut" if their closures have non-empty intersection.

We say that a dyadic cube $Q$ is "OK" if it satisfies the following condition:

$(\mathrm{OK})$ For every $y \in Q^{* *}$, there exist $\overline{\mathcal{A}}^{y}<\mathcal{A}$ and polynomials $\bar{P}_{\alpha}^{y} \in \mathcal{P}\left(\alpha \in \overline{\mathcal{A}}^{y}\right)$, with the following properties: 
$(\mathrm{OK} 1) \partial^{\beta} \bar{P}_{\alpha}^{y}(y)=\delta_{\beta \alpha}$ for all $\beta, \alpha \in \overline{\mathcal{A}}^{y}$.

$(\mathrm{OK} 2) \delta_{Q}^{|\beta|-|\alpha|}\left|\partial^{\beta} \bar{P}_{\alpha}^{y}(y)\right| \leq\left(a_{1}\right)^{-(m+2)}$ for all $\alpha \in \overline{\mathcal{A}}^{y}, \beta \in \mathcal{M}$ with $\beta \geq \alpha$.

(OK3) Given $\alpha \in \overline{\mathcal{A}}^{y}$ and $S \subset E$ with $\#(S) \leq k_{\text {old }}^{\#}$, there exists $\varphi_{\alpha}^{S, y} \in$ $C_{\ell o c}^{m, \omega}\left(\mathbb{R}^{n}\right)$, with

(a) $\left|\partial^{\beta} \varphi_{\alpha}^{S, y}\left(x^{\prime}\right)-\partial^{\beta} \varphi_{\alpha}^{S, y}\left(x^{\prime \prime}\right)\right| \leq\left(a_{1}\right)^{-(m+2)} \cdot \delta_{Q}^{|\alpha|-m-1} \cdot\left|x^{\prime}-x^{\prime \prime}\right|+\left(a_{1}\right)^{-(m+2)}$

$$
\text { for }|\beta|=m, x^{\prime}, x^{\prime \prime} \in \mathbb{R}^{n},\left|x^{\prime}-x^{\prime \prime}\right| \leq \delta_{Q} ;
$$

$$
\cdot a_{2} \cdot \delta_{Q}^{|\alpha|-m} \cdot \frac{\omega\left(\left|x^{\prime}-x^{\prime \prime}\right|\right)}{\omega\left(\delta_{Q}\right)}
$$

(b) $J_{x}\left(\varphi_{\alpha}^{S, y}\right) \in\left(a_{1}\right)^{-(m+2)} \cdot \delta_{Q}^{|\alpha|-m} \cdot\left(\omega\left(\delta_{Q}\right)\right)^{-1} \cdot \sigma(x)$ for all $x \in S$;

(c) $J_{y}\left(\varphi_{\alpha}^{S, y}\right)=\bar{P}_{\alpha}^{y}$.

Here, $k_{\text {old }}^{\#}$ is as in Lemma 8.1 and Section 9.

We say that a dyadic cube $Q$ is "almost OK" if either $Q$ is OK or $Q^{* *}$ contains at most one element of $E$.

We say that a dyadic cube $Q$ is a "CZ" or "Calderón-Zygmund" cube, if $Q$ is almost OK, but no dyadic cube $Q^{\prime}$ properly containing $Q$ is almost OK. Recall that, given any two dyadic cubes $Q_{1}, Q_{2}$, we always have one of the three alternatives: $Q_{1} \subseteq Q_{2}, Q_{2} \subset Q_{1}, Q_{1}$ and $Q_{2}$ disjoint. Consequently, any two distinct $\mathrm{CZ}$ cubes are disjoint. Moreover, since $E \subset \mathbb{R}^{n}$ is finite, any sufficiently small cube $Q$ can contain at most one element of $E$. Hence, any sufficiently small dyadic cube $Q$ is almost OK, and is therefore contained in a $\mathrm{CZ}$ cube.

Thus, we have the following easy result.

Lemma 11.1 The $\mathrm{CZ}$ cubes form a partition of $Q^{0}$ into finitely many dyadic subcubes.

Next, we prove that the CZ cubes have "good geometry".

Lemma 11.2 If two CZ cubes $Q, Q^{\prime}$ abut, then $\frac{1}{2} \delta_{Q} \leq \delta_{Q^{\prime}} \leq 2 \delta_{Q}$.

Proof: Suppose not. Without loss of generality, we may assume that $\delta_{Q} \leq \delta_{Q^{\prime}}$.

Since $\delta_{Q}=2^{-k} \delta_{Q^{0}}$ and $\delta_{Q^{\prime}}=2^{-k^{\prime}} \delta_{Q^{0}}$ for some integers $k, k^{\prime}$ (because $Q, Q^{\prime}$ are dyadic), we must have $\delta_{Q} \leq \frac{1}{4} \delta_{Q^{\prime}}$.

Hence, $Q \neq Q^{0}$, and the dyadic parent $Q^{+}$abuts $Q^{\prime}$ and satisfies

$$
\delta_{Q^{+}} \leq \frac{1}{2} \delta_{Q^{\prime}}
$$

Consequently, we have

$$
\left(Q^{+}\right)^{* *} \subset\left(Q^{\prime}\right)^{* *}
$$


We know that $Q^{\prime}$ is almost $\mathrm{OK}$, since it is a $\mathrm{CZ}$ cube. We will show that $Q^{+}$is almost OK. In fact, if $\left(Q^{\prime}\right)^{* *}$ contains at most one element of $E$, then the same is true of $\left(Q^{+}\right)^{* *}$ by $(5)$, and hence $Q^{+}$is almost OK, as claimed. If instead $\left(Q^{\prime}\right)^{* *}$ contains at least two distinct elements of $E$, then, since $Q^{\prime}$ is almost OK, we know that $Q^{\prime}$ is OK. In this case, we will show that $Q^{+}$is also OK. This will complete the proof that $Q^{+}$is almost OK.

To see that $Q^{+}$is OK whenever $Q^{\prime}$ is OK, we let $\overline{\mathcal{A}}^{y}, \bar{P}_{\alpha}^{y}\left(\alpha \in \overline{\mathcal{A}}^{y}\right)$ be as in $(\mathrm{OK} 1,2,3)$ for $y \in\left(Q^{\prime}\right)^{* *}$. Thus, $\overline{\mathcal{A}}^{y}, \bar{P}_{\alpha}^{y}\left(\alpha \in \overline{\mathcal{A}}^{y}\right)$ are defined for each $y \in\left(Q^{\prime}\right)^{* *}$, and so, in particular, for each $y \in\left(Q^{+}\right)^{* *}$, thanks to (5).

Moreover, conditions $(\mathrm{OK} 1,2,3)$ for $Q^{+}$follow from $(\mathrm{OK} 1,2,3)$ for $Q^{\prime}$, thanks to (5) and the following inequalities:

$$
\begin{aligned}
\delta_{Q^{+}}^{|\beta|-|\alpha|} & \leq \delta_{Q^{\prime}}^{|\beta|-|\alpha|} \quad \text { for } \beta \geq \alpha, \\
\delta_{Q^{\prime}}^{|\alpha|-m-1} & \leq \delta_{Q^{+}}^{|\alpha|-m-1} \quad \text { for } \alpha \in \mathcal{M}, \\
\delta_{Q^{\prime}}^{|\alpha|-m} & \leq \delta_{Q^{+}}^{|\alpha|-m} \quad \text { for } \alpha \in \mathcal{M}, \\
\left(\omega\left(\delta_{Q^{\prime}}\right)\right)^{-1} & \leq\left(\omega\left(\delta_{Q^{+}}\right)\right)^{-1} .
\end{aligned}
$$

These inequalities are immediate from (4) and the fact that $\omega$ is a regular modulus of continuity. Thus, (OK1,2,3) hold for $Q^{+}, \overline{\mathcal{A}}^{y}, \bar{P}_{\alpha}^{y}\left(\alpha \in \overline{\mathcal{A}}^{y}\right)$, completing the proof that $Q^{+}$is $\mathrm{OK}$ in this case. This also completes the proof of our claim that $Q^{+}$is almost OK.

However, $Q^{+}$cannot be almost $\mathrm{OK}$, since it is a dyadic cube properly containing the $\mathrm{CZ}$ cube $Q$. This contradiction completes the proof of Lemma 11.2.

As an easy consequence of Lemma 11.2, we have the following.

Lemma 11.3 For a small enough constant $c_{1}>0$ depending only on the dimension $n$, the following holds:

Suppose $x \in Q, x^{\prime} \in Q^{\prime}$, for $C Z$ cubes $Q$ and $Q^{\prime}$. If the balls $B\left(x, c_{1} \delta_{Q}\right)$ and $B\left(x^{\prime}, c_{1} \delta_{Q^{\prime}}\right)$ intersect, then the cubes $Q, Q^{\prime}$ coincide or abut.

Proof: Without loss of generality, we may suppose $\delta_{Q^{\prime}} \leq \delta_{Q}$.

If $B\left(x, c_{1} \delta_{Q}\right)$ intersects $B\left(x^{\prime}, c_{1} \delta_{Q^{\prime}}\right)$, then $\left|x-x^{\prime}\right| \leq c_{1} \delta_{Q}+c_{1} \delta_{Q^{\prime}} \leq 2 c_{1} \delta_{Q}$ hence $x^{\prime} \in\left\{y \in \mathbb{R}^{n}\right.$ : distance $\left.(y, Q) \leq 2 c_{1} \delta_{Q}\right\} \equiv \Omega$. However, if $c_{1}$ is a small enough constant depending only on the dimension $n$, then the set $\Omega \cap Q^{0}$ is covered by $Q$ and the $\mathrm{CZ}$ cubes that abut it, thanks to Lemma 11.2.

Consequently, $x^{\prime} \in Q^{\prime \prime}$, where $Q^{\prime \prime}$ is some $\mathrm{CZ}$ cube that coincides with or abuts $Q$. Since also $x^{\prime} \in Q^{\prime}$, the cubes $Q^{\prime}, Q^{\prime \prime}$ cannot be disjoint. Since two $\mathrm{CZ}$ cubes are either equal or disjoint, we must have $Q^{\prime}=Q^{\prime \prime}$. Hence, $Q^{\prime}$ and $Q$ coincide or abut. The proof of the lemma is complete.

Until the end of Section 16, we fix the cube $Q^{0}$ and the collection of CZ cubes. 


\section{Controlling Auxiliary Polynomials I}

We again place ourselves in the setting of Section 9, and we assume $(\mathrm{SU} 0, \ldots, 8)$. In this section only, we fix an integer $k_{1}^{\#}$, a dyadic cube $Q$, a point $y \in \mathbb{R}^{n}$, and a family of polynomials $P_{\alpha}^{y} \in \mathcal{P}$, indexed by $\alpha \in \mathcal{A}$; and we make the following assumptions.

$(\mathrm{CAP} 1) k^{\#} \geq(D+1) \cdot k_{1}^{\#}$, and $k_{1}^{\#} \geq(D+1) \cdot k_{\mathrm{old}}^{\#}$.

(CAP2) $y \in Q^{* * *}$.

(CAP3) $Q$ is properly contained in $Q^{0}$.

(CAP4) The $P_{\alpha}^{y}(\alpha \in \mathcal{A})$ satisfy conditions $(\mathrm{WL} 1)^{y},(\mathrm{WL} 2)^{y},(\mathrm{WL} 3)^{y}$. (See Lemma 10.3.)

(CAP5) $\left(a_{1}\right)^{-(m+1)} \leq \max _{\substack{\beta \in \mathcal{M} \\ \alpha \in \mathcal{A}}} \delta_{Q}^{|\beta|-|\alpha|}\left|\partial^{\beta} P_{\alpha}^{y}(y)\right| \leq 2^{m+1} \cdot\left(a_{1}\right)^{-(m+1)}$.

Note that $\mathcal{A}$ is non-empty, since the max in (CAP5) cannot be zero.

Our goal in this section is to show that the dyadic cube $Q^{+}$is OK. Let

$$
y^{\prime} \in\left(Q^{+}\right)^{* *} \text { be given. }
$$

Then $y, y^{\prime} \in Q^{* * *} \subset\left(Q^{0}\right)^{* * *} \subset B\left(y^{0}, a_{1}\right)$, by (11.2).

Applying Lemma 10.4 , with $k_{2}^{\#}=k_{\text {old }}^{\#}$, we obtain a family of polynomials $\tilde{P}_{\alpha}^{y^{\prime}} \in \mathcal{P}$, indexed by $\alpha \in \mathcal{A}$, with the following property.

(2) Given $\alpha \in \mathcal{A}$ and $S \subset E$ with $\#(S) \leq k_{\text {old }}^{\#}$, there exists $\varphi_{\alpha}^{S} \in C_{\ell o c}^{m, \omega}\left(\mathbb{R}^{n}\right)$, with

(a) $\left|\partial^{\beta} \varphi_{\alpha}^{S}\left(x^{\prime}\right)-\partial^{\beta} \varphi_{\alpha}^{S}\left(x^{\prime \prime}\right)\right| \leq C a_{2} \omega\left(\left|x^{\prime}-x^{\prime \prime}\right|\right)$ for $|\beta|=m, x^{\prime}, x^{\prime \prime} \in \mathbb{R}^{n}$, $\left|x^{\prime}-x^{\prime \prime}\right| \leq 1$

(b) $J_{x}\left(\varphi_{\alpha}^{S}\right) \in C \sigma(x)$ for all $x \in S$;

(c) $J_{y}\left(\varphi_{\alpha}^{S}\right)=P_{\alpha}^{y}$; and

(d) $J_{y^{\prime}}\left(\varphi_{\alpha}^{S}\right)=\tilde{P}_{\alpha}^{y^{\prime}}$.

We fix polynomials $\tilde{P}_{\alpha}^{y^{\prime}}$ satisfying (2). The basic properties of the $\tilde{P}_{\alpha}^{y^{\prime}}$, aside from (2), are as follows

Lemma 12.1 We have

$$
\begin{aligned}
& c \cdot\left(a_{1}\right)^{-(m+1)} \leq \max _{\substack{\beta \in \mathcal{M} \\
\alpha \in \mathcal{A}}} \delta_{Q}^{|\beta|-|\alpha|}\left|\partial^{\beta} \tilde{P}_{\alpha}^{y^{\prime}}\left(y^{\prime}\right)\right| \leq C \cdot\left(a_{1}\right)^{-(m+1)} ; \\
& \delta_{Q}^{|\beta|-|\alpha|}\left|\partial^{\beta} \tilde{P}_{\alpha}^{y^{\prime}}\left(y^{\prime}\right)\right| \leq C \cdot a_{1} \quad \text { for } \alpha \in \mathcal{A}, \beta \in \mathcal{M}, \beta>\alpha ; \\
& \left|\partial^{\alpha} \tilde{P}_{\alpha}^{y^{\prime}}\left(y^{\prime}\right)-1\right| \leq C \cdot a_{1} \quad \text { for } \alpha \in \mathcal{A} ; \text { and } \\
& \delta_{Q}^{|\beta|-|\alpha|}\left|\partial^{\beta} \tilde{P}_{\alpha}^{y^{\prime}}\left(y^{\prime}\right)\right| \leq C \quad \text { for } \beta, \alpha \in \mathcal{A} .
\end{aligned}
$$


Proof: We apply (2), with $S=$ empty set. Thus, for each $\alpha \in \mathcal{A}$, we obtain $\varphi_{\alpha} \in C_{\ell o c}^{m, \omega}\left(\mathbb{R}^{n}\right)$, with $J_{y}\left(\varphi_{\alpha}\right)=P_{\alpha}^{y}, J_{y^{\prime}}\left(\varphi_{\alpha}\right)=\tilde{P}_{\alpha}^{y^{\prime}}$, and $\left|\partial^{\beta} \varphi_{\alpha}\left(x^{\prime}\right)-\partial^{\beta} \varphi_{\alpha}\left(x^{\prime \prime}\right)\right| \leq C a_{2} \omega\left(\left|x^{\prime}-x^{\prime \prime}\right|\right)$ for $|\beta|=m, x^{\prime}, x^{\prime \prime} \in \mathbb{R}^{n},\left|x^{\prime}-x^{\prime \prime}\right| \leq 1$. For $\beta \in \mathcal{M}$, Taylor's theorem implies

$$
\begin{aligned}
\mid \partial^{\beta} \tilde{P}_{\alpha}^{y^{\prime}}\left(y^{\prime}\right)-\sum_{|\gamma| \leq m-|\beta|} & \frac{1}{\gamma !}\left(\partial^{\gamma+\beta} P_{\alpha}^{y}(y)\right) \cdot\left(y^{\prime}-y\right)^{\gamma} \mid= \\
& =\left|\partial^{\beta} \varphi_{\alpha}\left(y^{\prime}\right)-\sum_{|\gamma| \leq m-|\beta|} \frac{1}{\gamma !}\left(\partial^{\gamma+\beta} \varphi_{\alpha}(y)\right) \cdot\left(y^{\prime}-y\right)^{\gamma}\right| \\
& \leq C a_{2} \omega\left(\left|y^{\prime}-y\right|\right) \cdot\left|y^{\prime}-y\right|^{m-|\beta|}
\end{aligned}
$$

and

$$
\begin{aligned}
(8) \mid \partial^{\beta} P_{\alpha}^{y}(y)-\sum_{|\gamma| \leq m-|\beta|} & \frac{1}{\gamma !}\left(\partial^{\gamma+\beta} \tilde{P}_{\alpha}^{y^{\prime}}\left(y^{\prime}\right)\right) \cdot\left(y-y^{\prime}\right)^{\gamma} \mid= \\
& =\left|\partial^{\beta} \varphi_{\alpha}(y)-\sum_{|\gamma| \leq m-|\beta|} \frac{1}{\gamma !}\left(\partial^{\gamma+\beta} \varphi_{\alpha}\left(y^{\prime}\right)\right) \cdot\left(y-y^{\prime}\right)^{\gamma}\right| \\
& \leq C a_{2} \omega\left(\left|y^{\prime}-y\right|\right) \cdot\left|y^{\prime}-y\right|^{m-|\beta|} .
\end{aligned}
$$

In view of (CAP2) and (1), we have

$$
\left|y^{\prime}-y\right| \leq C \delta_{Q} \leq C \delta_{Q^{0}} \leq C a_{1}<1 .
$$

(We have also used the fact that $Q$ is dyadic, hence $Q \subseteq Q^{0}$; as well as (11.3) and (SU5).)

From (CAP5), we have

$$
\left|\partial^{\gamma+\beta} P_{\alpha}^{y}(y)\right| \leq 2^{m+1} \cdot\left(a_{1}\right)^{-(m+1)} \cdot \delta_{Q}^{|\alpha|-|\beta|-|\gamma|}
$$

for $\alpha \in \mathcal{A}, \beta \in \mathcal{M},|\gamma| \leq m-|\beta|$.

Putting (9) and (10) into (7), we find that

$$
\left|\partial^{\beta} \tilde{P}_{\alpha}^{y^{\prime}}\left(y^{\prime}\right)\right| \leq C\left(a_{1}\right)^{-(m+1)} \delta_{Q}^{|\alpha|-|\beta|}+C a_{2} \delta_{Q}^{m-|\beta|} \leq C^{\prime}\left(a_{1}\right)^{-(m+1)} \delta_{Q}^{|\alpha|-|\beta|}
$$

for $\alpha \in \mathcal{A}, \beta \in \mathcal{M}$.

(Here, we use the fact that $\delta_{Q} \leq \delta_{Q^{0}} \leq 1$, by virtue of (11.1)...(11.3), and also the fact that $\omega\left(\left|y^{\prime}-y\right|\right) \leq 1$ since $\left|y^{\prime}-y\right| \leq 1$ and $\omega$ is a regular modulus of continuity. See also (SU5,7).) 
On the other hand, if we put

$$
\Omega=\max _{\substack{\beta \in \mathcal{M} \\ \alpha \in \mathcal{A}}} \delta_{Q}^{|\beta|-|\alpha|}\left|\partial^{\beta} \tilde{P}_{\alpha}^{y^{\prime}}\left(y^{\prime}\right)\right|,
$$

then we have

$$
\left|\partial^{\gamma+\beta} \tilde{P}_{\alpha}^{y^{\prime}}\left(y^{\prime}\right)\right| \leq \Omega \delta_{Q}^{|\alpha|-|\beta|-|\gamma|} \text { for } \alpha \in \mathcal{A}, \beta \in \mathcal{M},|\gamma| \leq m-|\beta| .
$$

Putting (9) and (13) into (8), we find that

$$
\left|\partial^{\beta} P_{\alpha}^{y}(y)\right| \leq C \Omega \delta_{Q}^{|\alpha|-|\beta|}+C a_{2} \delta_{Q}^{m-|\beta|} \leq C \cdot[\Omega+1] \cdot \delta_{Q}^{|\alpha|-|\beta|}
$$

for $\alpha \in \mathcal{A}, \beta \in \mathcal{M}$.

Comparing (14) with (CAP5), we see that $C \cdot[\Omega+1] \geq\left(a_{1}\right)^{-(m+1)}$, hence $\Omega \geq c \cdot\left(a_{1}\right)^{-(m+1)}$. Together with (11) and (12), this proves conclusion (3).

Next, suppose $\alpha \in \mathcal{A}, \beta \in \mathcal{M}$ and $\beta>\alpha$. From (WL2) ${ }^{y}$ and Lemma 3.1, we have

$$
\left|\partial^{\gamma+\beta} P_{\alpha}^{y}(y)\right| \leq C a_{1} \quad \text { for }|\gamma| \leq m-|\beta| .
$$

Putting this and (9) into (7), and recalling (SU7), we have

$$
\left|\partial^{\beta} \tilde{P}_{\alpha}^{y^{\prime}}\left(y^{\prime}\right)\right| \leq C a_{1}+C a_{2} \leq C^{\prime} a_{1} .
$$

Since also $\delta_{Q} \leq 1$, and $|\beta| \geq|\alpha|$ for $\beta>\alpha$, we conclude that

$$
\delta_{Q}^{|\beta|-|\alpha|}\left|\partial^{\beta} \tilde{P}_{\alpha}^{y^{\prime}}\left(y^{\prime}\right)\right| \leq C^{\prime} a_{1} \quad \text { for } \alpha \in \mathcal{A}, \beta \in \mathcal{M}, \beta>\alpha .
$$

Thus, we have proven conclusion (4).

Next, suppose $\alpha \in \mathcal{A}$, and take $\beta=\alpha$. By (SU0), we have $\gamma+\beta \in \mathcal{A}$ for $|\gamma| \leq m-|\beta|$. Hence, $(\mathrm{WL} 1)^{y}$ gives $\partial^{\gamma+\beta} P_{\alpha}^{y}(y)=\delta_{\gamma+\beta, \alpha}=\delta_{\gamma, 0}$, and therefore (7) yields

$$
\left|\partial^{\alpha} \tilde{P}_{\alpha}^{y^{\prime}}\left(y^{\prime}\right)-1\right| \leq C a_{2} \leq C a_{1}, \text { thanks to (SU7) }
$$

This proves conclusion (5).

Next, suppose $\alpha, \beta \in \mathcal{A}$. Then, again (SU0) gives $\gamma+\beta \in \mathcal{A}$ for $|\gamma| \leq$ $m-|\beta|$; hence, $(\mathrm{WL} 1)^{y}$ gives $\partial^{\beta+\gamma} P_{\alpha}^{y}(y)=\delta_{\beta+\gamma, \alpha}$.

In particular, we have

$$
\left|\partial^{\gamma+\beta} P_{\alpha}^{y}(y)\right| \leq \delta_{Q}^{|\alpha|-|\beta|-|\gamma|} \text { for }|\gamma| \leq m-|\beta| .
$$

Putting this and (9) into (7), we find that

$$
\left|\partial^{\beta} \tilde{P}_{\alpha}^{y^{\prime}}\left(y^{\prime}\right)\right| \leq C \delta_{Q}^{|\alpha|-|\beta|}+C a_{2} \delta_{Q}^{m-|\beta|} \leq C^{\prime} \delta_{Q}^{|\alpha|-|\beta|} .
$$

This proves conclusion (6). The proof of Lemma 12.1 is complete 
Define a matrix

$$
\tilde{M}=\left(\tilde{M}_{\beta \alpha}\right)_{\beta, \alpha \in \mathcal{A}}
$$

by setting

$$
\tilde{M}_{\beta \alpha}=\delta_{Q}^{|\beta|-|\alpha|} \partial^{\beta} \tilde{P}_{\alpha}^{y^{\prime}}\left(y^{\prime}\right) \text { for } \beta, \alpha \in \mathcal{A} .
$$

From (4), (5), (6) we see that

$$
\left[\begin{array}{l}
\left|\tilde{M}_{\beta \alpha}\right| \leq C a_{1} \text { for } \beta>\alpha(\beta, \alpha \in \mathcal{A}) \\
\left|\tilde{M}_{\alpha \alpha}-1\right| \leq C a_{1} \text { for } \alpha \in \mathcal{A} \\
\left|\tilde{M}_{\beta \alpha}\right| \leq C \text { for all } \beta, \alpha \in \mathcal{A}
\end{array}\right.
$$

That is, $\tilde{M}$ lies within distance $C a_{1}$ of a triangular matrix with 1 's on the main diagonal, and with entries bounded by $C$.

It follows that the inverse matrix

$$
M=\left(M_{\alpha^{\prime} \alpha}\right)_{\alpha^{\prime}, \alpha \in \mathcal{A}}
$$

satisfies the same property, i.e.,

$$
\begin{aligned}
\left|M_{\alpha^{\prime} \alpha}\right| \leq C a_{1} & \text { if } \alpha^{\prime}>\alpha\left(\alpha^{\prime}, \alpha \in \mathcal{A}\right) \\
\left|M_{\alpha \alpha}-1\right| \leq C a_{1} & \text { if } \alpha \in \mathcal{A}
\end{aligned}
$$

and

$$
\left|M_{\alpha^{\prime} \alpha}\right| \leq C \text { for all } \alpha^{\prime}, \alpha \in \mathcal{A} \text {. }
$$

By definition, we have

$$
\sum_{\alpha^{\prime} \in \mathcal{A}} \tilde{M}_{\beta \alpha^{\prime}} M_{\alpha^{\prime} \alpha}=\delta_{\beta \alpha} \quad \text { for all } \beta, \alpha \in \mathcal{A} .
$$

That is,

$$
\sum_{\alpha^{\prime} \in \mathcal{A}} \delta_{Q}^{|\beta|-\left|\alpha^{\prime}\right|} \partial^{\beta} \tilde{P}_{\alpha^{\prime}}^{y^{\prime}}\left(y^{\prime}\right) \cdot M_{\alpha^{\prime} \alpha}=\delta_{\beta \alpha} \quad \text { for all } \beta, \alpha \in \mathcal{A}
$$

We define the polynomials $\check{P}_{\alpha}^{y^{\prime}} \in \mathcal{P}$ by setting

$$
\check{P}_{\alpha}^{y^{\prime}}=\delta_{Q}^{|\alpha|} \cdot \sum_{\alpha^{\prime} \in \mathcal{A}} \delta_{Q}^{-\left|\alpha^{\prime}\right|} \tilde{P}_{\alpha^{\prime}}^{y^{\prime}} \cdot M_{\alpha^{\prime} \alpha} \quad \text { for all } \alpha \in \mathcal{A} .
$$


The basic properties of the $\check{P}_{\alpha}^{y^{\prime}}$ are as follows.

Lemma 12.2 We have

(23) $\partial^{\beta} \check{P}_{\alpha}^{y^{\prime}}\left(y^{\prime}\right)=\delta_{\beta \alpha}$ for all $\beta, \alpha \in \mathcal{A}$;

(24) $c \cdot\left(a_{1}\right)^{-(m+1)}<\max _{\substack{\beta \in \mathcal{M} \\ \alpha \in \mathcal{A}}} \delta_{Q}^{|\beta|-|\alpha|}\left|\partial^{\beta} \check{P}_{\alpha}^{y^{\prime}}\left(y^{\prime}\right)\right|<C \cdot\left(a_{1}\right)^{-(m+1)} ;$

(25) $\delta_{Q}^{|\beta|-|\alpha|}\left|\partial^{\beta} \check{P}_{\alpha}^{y^{\prime}}\left(y^{\prime}\right)\right| \leq C \cdot\left(a_{1}\right)^{-m}$ for all $\alpha \in \mathcal{A}, \beta \in \mathcal{M}$ with $\beta>\alpha$;

(26) Given $\alpha \in \mathcal{A}$ and $S \subset E$ with $\#(S) \leq k_{\text {old }}^{\#}$, there exists $\check{\varphi}_{\alpha}^{S} \in C_{\text {loc }}^{m, \omega}\left(\mathbb{R}^{n}\right)$, with

(a) $\left|\partial^{\beta} \check{\varphi}_{\alpha}^{S}\left(x^{\prime}\right)-\partial^{\beta} \check{\varphi}_{\alpha}^{S}\left(x^{\prime \prime}\right)\right| \leq C a_{2} \delta_{Q}^{|\alpha|-m} \cdot \frac{\omega\left(\left|x^{\prime}-x^{\prime \prime}\right|\right)}{\omega\left(\delta_{Q}\right)}$

for $|\beta|=m, x^{\prime}, x^{\prime \prime} \in \mathbb{R}^{n},\left|x^{\prime}-x^{\prime \prime}\right| \leq 100 \delta_{Q} ;$

(b) $J_{x}\left(\check{\varphi}_{\alpha}^{S}\right) \in C \delta_{Q}^{|\alpha|-m}\left(\omega\left(\delta_{Q}\right)\right)^{-1} \sigma(x)$ for all $x \in S$; and

(c) $J_{y^{\prime}}\left(\check{\varphi}_{\alpha}^{S}\right)=\check{P}_{\alpha}^{y^{\prime}}$.

Proof: Conclusion (23) is immediate from (21) and (22).

From (22), we have

$$
\left[\delta_{Q}^{|\beta|-|\alpha|} \partial^{\beta} \check{P}_{\alpha}^{y^{\prime}}\left(y^{\prime}\right)\right]=\sum_{\alpha^{\prime} \in \mathcal{A}}\left[\delta_{Q}^{|\beta|-\left|\alpha^{\prime}\right|} \partial^{\beta} \tilde{P}_{\alpha^{\prime}}^{y^{\prime}}\left(y^{\prime}\right)\right] \cdot M_{\alpha^{\prime} \alpha}
$$

for $\beta \in \mathcal{M}, \alpha \in \mathcal{A}$.

Since $M$ and $\tilde{M}$ are inverse matrices, (27) implies

$$
\left[\delta_{Q}^{|\beta|-|\alpha|} \partial^{\beta} \tilde{P}_{\alpha}^{y^{\prime}}\left(y^{\prime}\right)\right]=\sum_{\alpha^{\prime} \in \mathcal{A}}\left[\delta_{Q}^{|\beta|-\left|\alpha^{\prime}\right|} \partial^{\beta} \check{P}_{\alpha^{\prime}}^{y^{\prime}}\left(y^{\prime}\right)\right] \cdot \tilde{M}_{\alpha^{\prime} \alpha}
$$

for $\beta \in \mathcal{M}, \alpha \in \mathcal{A}$.

From (16), (19), (27), (28), we conclude that

$$
\begin{aligned}
c \cdot \max _{\substack{\beta \in \mathcal{M} \\
\alpha \in \mathcal{A}}}\left[\delta_{Q}^{|\beta|-|\alpha|}\left|\partial^{\beta} \tilde{P}_{\alpha}^{y^{\prime}}\left(y^{\prime}\right)\right|\right] & \leq \max _{\substack{\beta \in \mathcal{M} \\
\alpha \in \mathcal{A}}}\left[\delta_{Q}^{|\beta|-|\alpha|}\left|\partial^{\beta} \check{P}_{\alpha}^{y^{\prime}}\left(y^{\prime}\right)\right|\right] \\
& \leq C \cdot \max _{\substack{\beta \in \mathcal{M} \\
\alpha \in \mathcal{A}}}\left[\delta_{Q}^{|\beta|-|\alpha|}\left|\partial^{\beta} \tilde{P}_{\alpha}^{y^{\prime}}\left(y^{\prime}\right)\right|\right] .
\end{aligned}
$$

Together with (3), this proves conclusion (24).

Next, suppose $\beta \in \mathcal{M}, \alpha \in \mathcal{A}$, with $\beta>\alpha$. Then, for each $\alpha^{\prime} \in \mathcal{A}$, we have either $\beta>\alpha^{\prime}$ or $\alpha^{\prime}>\alpha$. If $\beta>\alpha^{\prime}$, then (4) and (19) yield

$$
\left|\left[\delta_{Q}^{|\beta|-\left|\alpha^{\prime}\right|} \partial^{\beta} \tilde{P}_{\alpha^{\prime}}^{y^{\prime}}\left(y^{\prime}\right)\right] \cdot M_{\alpha^{\prime} \alpha}\right| \leq C a_{1} \leq C\left(a_{1}\right)^{-m} \text { by (SU5). }
$$


If instead $\alpha^{\prime}>\alpha$, then (3) and (17) yield

$$
\left|\left[\delta_{Q}^{|\beta|-\left|\alpha^{\prime}\right|} \partial^{\beta} \tilde{P}_{\alpha^{\prime}}^{y^{\prime}}\left(y^{\prime}\right)\right] \cdot M_{\alpha^{\prime} \alpha}\right| \leq C \cdot\left(a_{1}\right)^{-(m+1)} \cdot C \cdot\left(a_{1}\right)=C^{\prime} \cdot\left(a_{1}\right)^{-m} .
$$

Consequently, (27) implies conclusion (25).

Finally, let $S \subset E$, with $\#(S) \leq k_{\text {old }}^{\#}$. For each $\alpha \in \mathcal{A}$, let $\varphi_{\alpha}^{S} \in C_{\text {loc }}^{m, \omega}\left(\mathbb{R}^{n}\right)$ be as in (2). We define

$$
\check{\varphi}_{\alpha}^{S}=\delta_{Q}^{|\alpha|} \sum_{\alpha^{\prime} \in \mathcal{A}} \delta_{Q}^{-\left|\alpha^{\prime}\right|} \varphi_{\alpha^{\prime}}^{S} M_{\alpha^{\prime} \alpha} \quad \text { for } \alpha \in \mathcal{A} .
$$

Thus, $\check{\varphi}_{\alpha}^{S} \in C_{\ell o c}^{m, \omega}\left(\mathbb{R}^{n}\right)$. Also, for $|\beta|=m, x^{\prime}, x^{\prime \prime} \in \mathbb{R}^{n},\left|x^{\prime}-x^{\prime \prime}\right| \leq 100 \delta_{Q}$, (2)(a) and (19) show that

$$
\begin{aligned}
\mid \partial^{\beta} \check{\varphi}_{\alpha}^{S}\left(x^{\prime}\right) & -\partial^{\beta} \check{\varphi}_{\alpha}^{S}\left(x^{\prime \prime}\right)\left|\leq \delta_{Q}^{|\alpha|} \sum_{\alpha^{\prime} \in \mathcal{A}} \delta_{Q}^{-\left|\alpha^{\prime}\right|}\right| \partial^{\beta} \varphi_{\alpha^{\prime}}^{S}\left(x^{\prime}\right)-\partial^{\beta} \varphi_{\alpha^{\prime}}^{S}\left(x^{\prime \prime}\right)|\cdot| M_{\alpha^{\prime} \alpha} \mid \\
& \leq \sum_{\alpha^{\prime} \in \mathcal{A}} \delta_{Q}^{|\alpha|-\left|\alpha^{\prime}\right|} \cdot C a_{2} \omega\left(\left|x^{\prime}-x^{\prime \prime}\right|\right) \cdot C \leq C^{\prime} \delta_{Q}^{|\alpha|-m} a_{2} \omega\left(\left|x^{\prime}-x^{\prime \prime}\right|\right) \\
& \leq C^{\prime} a_{2} \delta_{Q}^{|\alpha|-m} \frac{\omega\left(\left|x^{\prime}-x^{\prime \prime}\right|\right)}{\omega\left(\delta_{Q}\right)}
\end{aligned}
$$

which shows that the $\check{\varphi}_{\alpha}^{S}$ satisfy $(26)(\mathrm{a})$.

From (2)(b), (19), (29) we see that for $x \in S$ we have

$$
\begin{aligned}
J_{x}\left(\check{\varphi}_{\alpha}^{S}\right) \in \delta_{Q}^{|\alpha|} \sum_{\alpha^{\prime} \in \mathcal{A}} \delta_{Q}^{-\left|\alpha^{\prime}\right|}\left|M_{\alpha^{\prime} \alpha}\right| \cdot C \sigma(x) & \subseteq \sum_{\alpha^{\prime} \in \mathcal{A}} C \delta_{Q}^{|\alpha|-\left|\alpha^{\prime}\right|} \sigma(x) \\
& \subseteq C \delta_{Q}^{|\alpha|-m} \sigma(x) \subseteq \frac{C \delta_{Q}^{|\alpha|-m}}{\omega\left(\delta_{Q}\right)} \sigma(x) .
\end{aligned}
$$

This shows that the $\breve{\varphi}_{\alpha}^{S}$ satisfy condition (26)(b).

For each $\alpha \in \mathcal{A},(2)(\mathrm{d}),(22),(29)$ together show that the $\check{\varphi}_{\alpha}^{S}$ satisfy condition $(26)(\mathrm{c})$.

Thus, given $\alpha \in \mathcal{A}, S \subset E$ with $\#(S) \leq k_{\text {old }}^{\#}$, we have exhibited a function $\check{\varphi}_{\alpha}^{S} \in C_{\ell o c}^{m, \omega}\left(\mathbb{R}^{n}\right)$ satisfying $(26)(\mathrm{a}),(\mathrm{b}),(\mathrm{c})$. This completes the proof of conclusion (26), hence also that of Lemma 12.2.

Next, we pick $\bar{\beta} \in \mathcal{M}$ and $\bar{\alpha} \in \mathcal{A}$ to maximize $\delta_{Q}^{|\bar{\beta}|-|\bar{\alpha}|}\left|\partial^{\bar{\beta}} \check{P}_{\bar{\alpha}}^{y^{\prime}}\left(y^{\prime}\right)\right|$. By definition of $\bar{\beta}, \bar{\alpha}$, and by (24), we have

$$
\begin{aligned}
& c \cdot\left(a_{1}\right)^{-(m+1)}<\delta_{Q}^{|\bar{\beta}|-|\bar{\alpha}|}\left|\partial^{\bar{\beta}} \check{P}_{\bar{\alpha}}^{y^{\prime}}\left(y^{\prime}\right)\right|<C \cdot\left(a_{1}\right)^{-(m+1)} ; \\
& \delta_{Q}^{|\beta|-|\alpha|}\left|\partial^{\beta} \check{P}_{\alpha}^{y^{\prime}}\left(y^{\prime}\right)\right| \leq \delta_{Q}^{|\bar{\beta}|-|\bar{\alpha}|}\left|\partial^{\bar{\beta}} \check{P}_{\bar{\alpha}}^{y^{\prime}}\left(y^{\prime}\right)\right| \quad \text { for all } \beta \in \mathcal{M}, \alpha \in \mathcal{A} ; \\
& \bar{\beta} \in \mathcal{M}, \bar{\alpha} \in \mathcal{A} .
\end{aligned}
$$


If $\bar{\beta} \in \mathcal{A}$, then (23) gives $\delta_{Q}^{|\bar{\beta}|-|\bar{\alpha}|}\left|\partial^{\bar{\beta}} \check{P}_{\bar{\alpha}}^{y^{\prime}}\left(y^{\prime}\right)\right|=\delta_{\bar{\beta} \bar{\alpha}} \leq 1$, contradicting (30) thanks to (SU5). Hence,

$$
\bar{\beta} \notin \mathcal{A} \text {. }
$$

In particular, $\bar{\beta} \neq \bar{\alpha}$. If $\bar{\beta}>\bar{\alpha}$, then (30) contradicts (25). Hence,

$$
\bar{\beta}<\bar{\alpha} \text {. }
$$

Now define

$$
\begin{aligned}
& \overline{\mathcal{A}}^{y^{\prime}}=(\mathcal{A} \backslash\{\bar{\alpha}\}) \cup\{\bar{\beta}\}, \\
& \stackrel{+}{P_{\alpha}^{y^{\prime}}}=\check{P}_{\alpha}^{y^{\prime}} \quad \text { for all } \alpha \in \mathcal{A} \backslash\{\bar{\alpha}\}, \\
& \stackrel{+}{P}_{\bar{\beta}}^{y^{\prime}}=\check{P}_{\bar{\alpha}}^{y^{\prime}} /\left(\partial^{\bar{\beta}} \check{P}_{\bar{\alpha}}^{y^{\prime}}\left(y^{\prime}\right)\right) .
\end{aligned}
$$

The denominator in (37) is non-zero, thanks to (30). We have defined $\stackrel{+}{P}_{\alpha}^{y^{\prime}} \in \mathcal{P}$ for all $\alpha \in \overline{\mathcal{A}}^{y^{\prime}}$, as we see from (35), (36), (37).

In view of $(32), \ldots,(35)$, the least element of the symmetric difference $\overline{\mathcal{A}}^{y^{\prime}} \Delta \mathcal{A}$ is $\bar{\beta}$, which lies in $\overline{\mathcal{A}}^{y^{\prime}}$. Hence, by definition of our ordering on sets of multi-indices, we have

$$
\overline{\mathcal{A}}^{y^{\prime}}<\mathcal{A} \text {. }
$$

The basic properties of the $\stackrel{+}{P}_{\alpha}^{y^{\prime}}$ are as follows.

Lemma 12.3 We have

(39) $\partial^{\beta} \stackrel{+}{P} \overline{\bar{\beta}}_{\bar{\beta}}^{y^{\prime}}=\delta_{\beta \bar{\beta}}$ for all $\beta \in \overline{\mathcal{A}}^{y^{\prime}}$;

(40) $\partial^{\beta} \stackrel{+}{P}_{\alpha}^{y^{\prime}}\left(y^{\prime}\right)=\delta_{\beta \alpha}$ for all $\beta, \alpha \in \overline{\mathcal{A}}^{y^{\prime}} \backslash\{\bar{\beta}\}$;

(41) $\delta_{Q}^{|\beta|-|\alpha|}\left|\partial^{\beta} \stackrel{+}{P}{ }_{\alpha}^{y^{\prime}}\left(y^{\prime}\right)\right| \leq C \cdot\left(a_{1}\right)^{-(m+1)}$ for all $\beta \in \mathcal{M}, \alpha \in \overline{\mathcal{A}}^{y^{\prime}} ;$

(42) $\delta_{Q}^{|\beta|-|\bar{\beta}|}\left|\partial^{\beta} \stackrel{+}{P} \bar{\beta}_{\bar{\beta}}^{y^{\prime}}\left(y^{\prime}\right)\right| \leq 1$ for all $\beta \in \mathcal{M} ;$ and

(43) Given $\alpha \in \overline{\mathcal{A}}^{y^{\prime}}$ and $S \subset E$ with $\#(S) \leq k_{\text {old }}^{\#}$, there exists $\stackrel{\varphi}{\varphi}_{\alpha}^{S} \in$ $C_{\ell o c}^{m, \omega}\left(\mathbb{R}^{n}\right)$, with
(a) $\left|\partial^{\beta} \stackrel{+}{\varphi}_{\alpha}^{S}\left(x^{\prime}\right)-\partial^{\beta} \stackrel{+}{\varphi}_{\alpha}^{S}\left(x^{\prime \prime}\right)\right| \leq C a_{2} \delta_{Q}^{|\alpha|-m} \frac{\omega\left(\left|x^{\prime}-x^{\prime \prime}\right|\right)}{\omega\left(\delta_{Q}\right)}$ for $|\beta|=m, x^{\prime}, x^{\prime \prime} \in$ $\mathbb{R}^{n},\left|x^{\prime}-x^{\prime \prime}\right| \leq 100 \delta_{Q}$
(b) $J_{x}\left(\stackrel{+}{\varphi_{\alpha}^{S}}\right) \in \frac{C \delta_{Q}^{|\alpha|-m}}{\omega\left(\delta_{Q}\right)} \sigma(x)$ for all $x \in S$; and
(c) $J_{y^{\prime}}(\stackrel{+}{\varphi} \underset{\alpha}{S})=\stackrel{+}{y_{\alpha}^{\prime}}$ 
Proof: To check (39), we note that for $\beta \in \overline{\mathcal{A}}^{y^{\prime}} \backslash\{\bar{\beta}\}=\mathcal{A} \backslash\{\bar{\alpha}\}$, we have from (37) that

$$
\partial^{\beta} \stackrel{+}{P}{\stackrel{y^{\prime}}{\prime}}^{\prime}\left(y^{\prime}\right)=\partial^{\beta} \check{P}_{\bar{\alpha}}^{y^{\prime}}\left(y^{\prime}\right) /\left(\partial^{\bar{\beta}} \check{P}_{\bar{\alpha}}^{y^{\prime}}\left(y^{\prime}\right)\right)=0 \text {, thanks to }(23) .
$$

On the other hand, (37) gives also that

$$
\partial^{\bar{\beta}} \stackrel{+}{P}{\stackrel{y^{\prime}}{\prime}}^{\prime}\left(y^{\prime}\right)=\partial^{\bar{\beta}} \check{P}_{\bar{\alpha}}^{y^{\prime}}\left(y^{\prime}\right) /\left(\partial^{\bar{\beta}} \check{P}_{\bar{\alpha}}^{y^{\prime}}\left(y^{\prime}\right)\right)=1 .
$$

This proves conclusion (39). Conclusion (40) is immediate from (23) and (36), since $\overline{\mathcal{A}}^{y^{\prime}} \backslash\{\bar{\beta}\}=\mathcal{A} \backslash\{\bar{\alpha}\}$.

Similarly, conclusion (41) for $\alpha \in \overline{\mathcal{A}}^{y^{\prime}} \backslash\{\bar{\beta}\}=\mathcal{A} \backslash\{\bar{\alpha}\}$ follows at once from (24) and (36).

On the other hand, (31) and (37) show that

$$
\delta_{Q}^{|\beta|-|\bar{\beta}|}\left|\partial^{\beta} \stackrel{+}{P}_{\bar{\beta}}^{y^{\prime}}\left(y^{\prime}\right)\right|=\left|\left[\delta_{Q}^{|\beta|-|\bar{\alpha}|} \partial^{\beta} \check{P}_{\bar{\alpha}}^{y^{\prime}}\left(y^{\prime}\right)\right] /\left[\delta_{Q}^{|\bar{\beta}|-|\bar{\alpha}|} \partial^{\bar{\beta}} \check{P}_{\bar{\alpha}}^{y^{\prime}}\left(y^{\prime}\right)\right]\right| \leq 1
$$

for all $\beta \in \mathcal{M}$. This proves conclusion (42), and also shows that conclusion (41) holds for $\alpha=\bar{\beta}$. The proof of (41) is complete.

For $\alpha \in \overline{\mathcal{A}}^{y^{\prime}} \backslash\{\bar{\beta}\}=\overline{\mathcal{A}} \backslash\{\bar{\alpha}\}$, conclusion (43) is immediate from (26) and (36). It remains to prove (43) in the case $\alpha=\bar{\beta}$.

Suppose $\alpha=\bar{\beta}$, and let $S \subset E$, with $\#(S) \leq k_{\text {old }}^{\#}$. We let $\check{\varphi}_{\bar{\alpha}}^{S} \in C_{\text {loc }}^{m, \omega}\left(\mathbb{R}^{n}\right)$ be as in (26), and we define

$$
\stackrel{+}{\varphi}_{\bar{\beta}}^{S}=\check{\varphi}_{\bar{\alpha}}^{S} /\left(\partial^{\bar{\beta}} \check{P}_{\bar{\alpha}}^{y^{\prime}}\left(y^{\prime}\right)\right) .
$$

From (26)(a) and (30), we have, for $|\beta|=m, x^{\prime}, x^{\prime \prime} \in \mathbb{R}^{n}$ with $\left|x^{\prime}-x^{\prime \prime}\right| \leq$ $100 \delta_{Q}$, that

$$
\begin{aligned}
& \left|\partial^{\beta} \stackrel{+}{\varphi}{ }_{\bar{\beta}}^{S}\left(x^{\prime}\right)-\partial^{\beta} \stackrel{+}{\varphi}_{\bar{\beta}}^{S}\left(x^{\prime \prime}\right)\right|=\left|\partial^{\bar{\beta}} \check{P}_{\bar{\alpha}}^{y^{\prime}}\left(y^{\prime}\right)\right|^{-1} \cdot\left|\partial^{\beta} \check{\varphi}_{\bar{\alpha}}^{S}\left(x^{\prime}\right)-\partial^{\beta} \check{\varphi}_{\bar{\alpha}}^{S}\left(x^{\prime \prime}\right)\right| \\
& \quad \leq\left[C a_{1}^{(m+1)} \delta_{Q}^{|\bar{\beta}|-|\bar{\alpha}|}\right] \cdot\left[C a_{2} \delta_{Q}^{|\bar{\alpha}|-m} \frac{\omega\left(\left|x^{\prime}-x^{\prime \prime}\right|\right)}{\omega\left(\delta_{Q}\right)}\right] \leq C a_{2} \delta_{Q}^{|\bar{\beta}|-m} \frac{\omega\left(\left|x^{\prime}-x^{\prime \prime}\right|\right)}{\omega\left(\delta_{Q}\right)} .
\end{aligned}
$$

This proves conclusion (43)(a) for $\alpha=\bar{\beta}$.

From (26)(b) and (30), we have for $x \in S$ that

$$
\begin{aligned}
& J_{x}\left({ }_{\varphi}^{+} \bar{S}_{\bar{\beta}}\right)=\left[\partial^{\bar{\beta}} \check{P}_{\bar{\alpha}}^{y^{\prime}}\left(y^{\prime}\right)\right]^{-1} J_{x}\left(\check{\varphi}_{\bar{\alpha}}^{S}\right) \in\left[\partial^{\bar{\beta}} \check{P}_{\bar{\alpha}}^{y^{\prime}}\left(y^{\prime}\right)\right]^{-1} \cdot C \delta_{Q}^{|\bar{\alpha}|-m}\left(\omega\left(\delta_{Q}\right)\right)^{-1} \sigma(x) \\
& \quad \subseteq\left[C \cdot\left(a_{1}\right)^{m+1} \cdot \delta_{Q}^{|\bar{\beta}|-|\bar{\alpha}|}\right] \cdot C \delta_{Q}^{|\overline{\mid}|-m}\left(\omega\left(\delta_{Q}\right)\right)^{-1} \sigma(x) \subseteq C \delta_{Q}^{|\bar{\beta}|-m}\left(\omega\left(\delta_{Q}\right)\right)^{-1} \sigma(x) .
\end{aligned}
$$

This proves conclusion (43)(b) for $\beta=\bar{\alpha}$.

Finally, comparing (37) with (44), and recalling (26)(c), we obtain conclusion (43)(c) for $\beta=\bar{\alpha}$. Thus, (43) holds for $\beta=\bar{\alpha}$. The proof of Lemma 12.3 is complete. 
Next, we define polynomials $\bar{P}_{\alpha}^{y^{\prime}} \in \mathcal{P}\left(\alpha \in \overline{\mathcal{A}}^{y^{\prime}}\right)$, by setting

$$
\begin{aligned}
& \bar{P}_{\bar{\beta}}^{y^{\prime}}=\stackrel{+}{P_{\bar{\beta}}^{y^{\prime}}}, \quad \text { and } \\
& \bar{P}_{\alpha}^{y^{\prime}}=\stackrel{+}{P_{\alpha}^{y^{\prime}}}-\left[\partial^{\bar{\beta}} \stackrel{+}{P}_{\alpha}^{y^{\prime}}\left(y^{\prime}\right)\right] \cdot \stackrel{+}{P}_{\bar{\beta}}^{y^{\prime}} \quad \text { for all } \alpha \in \overline{\mathcal{A}} \backslash\{\bar{\alpha}\} .
\end{aligned}
$$

The basic properties of these polynomials are as follows.

Lemma 12.4 We have

(47) $\partial^{\beta} \bar{P}_{\alpha}^{y^{\prime}}\left(y^{\prime}\right)=\delta_{\beta \alpha}$ for all $\beta, \alpha \in \overline{\mathcal{A}}^{y^{\prime}}$;

(48) $\delta_{Q}^{|\beta|-|\alpha|}\left|\partial^{\beta} \bar{P}_{\alpha}^{y^{\prime}}\left(y^{\prime}\right)\right| \leq C \cdot\left(a_{1}\right)^{-(m+1)}$ for all $\beta \in \mathcal{M}, \alpha \in \overline{\mathcal{A}}^{y^{\prime}} ;$ and

(49) Given $\alpha \in \overline{\mathcal{A}}^{y^{\prime}}$ and $S \subset E$ with $\#(S) \leq k_{\text {old }}^{\#}$, there exists $\bar{\varphi}_{\alpha}^{S} \in C_{\text {loc }}^{m, \omega}\left(\mathbb{R}^{n}\right)$ with

(a) $\left|\partial^{\beta} \bar{\varphi}_{\alpha}^{S}\left(x^{\prime}\right)-\partial^{\beta} \bar{\varphi}_{\alpha}^{S}\left(x^{\prime \prime}\right)\right| \leq C \cdot\left(a_{1}\right)^{-(m+1)} \cdot a_{2} \cdot \delta_{Q}^{|\alpha|-m} \cdot \frac{\omega\left(\left|x^{\prime}-x^{\prime \prime}\right|\right)}{\omega\left(\delta_{Q}\right)}$ for $|\beta|=m, x^{\prime}, x^{\prime \prime} \in \mathbb{R}^{n},\left|x^{\prime}-x^{\prime \prime}\right| \leq 100 \delta_{Q}$;

(b) $J_{x}\left(\bar{\varphi}_{\alpha}^{S}\right) \in C \cdot\left(a_{1}\right)^{-(m+1)} \cdot \frac{\delta_{Q}^{|\alpha|-m}}{\omega\left(\delta_{Q}\right)} \sigma(x)$ for all $x \in S$; and

(c) $J_{y^{\prime}}\left(\bar{\varphi}_{\alpha}^{S}\right)=\bar{P}_{\alpha}^{y^{\prime}}$.

Proof: For $\alpha=\bar{\beta}$, conclusion (47) is immediate from (39) and (45).

For $\alpha \in \overline{\mathcal{A}}^{y^{\prime}} \backslash\{\bar{\beta}\}=\mathcal{A} \backslash\{\bar{\alpha}\},(46)$ gives

$$
\partial^{\beta} \bar{P}_{\alpha}^{y^{\prime}}\left(y^{\prime}\right)=\partial^{\beta} \stackrel{+}{P_{\alpha}^{y^{\prime}}}\left(y^{\prime}\right)-\left[\partial^{\bar{\beta}} \stackrel{+}{P}_{\alpha}^{y^{\prime}}\left(y^{\prime}\right)\right] \cdot \partial^{\beta} \stackrel{+}{P_{\bar{\beta}}^{y^{\prime}}}\left(y^{\prime}\right) \quad \text { for all } \beta \in \mathcal{M}
$$

If $\beta \in \mathcal{A} \backslash\{\bar{\alpha}\}$, then (39), (40) give $\partial^{\beta} \stackrel{+}{P_{\alpha}^{y^{\prime}}}\left(y^{\prime}\right)=\delta_{\beta \alpha}$ and $\partial^{\beta} \stackrel{+}{P_{\bar{\beta}}^{y^{\prime}}}\left(y^{\prime}\right)=0$; hence (50) gives conclusion (47) in this case.

If instead, $\beta=\bar{\beta}$, then from (39) we have $\partial^{\beta} \stackrel{+}{P} \underset{\bar{\beta}}{y^{\prime}}\left(y^{\prime}\right)=1$; hence (50) gives $\partial^{\beta} \bar{P}_{\alpha}^{y^{\prime}}\left(y^{\prime}\right)=0=\delta_{\beta \alpha}$, so again (47) holds in this case.

Thus, conclusion (47) holds in all cases.

Next, conclusion (48) holds for $\alpha=\bar{\beta}$, by (41) and (45). Suppose $\alpha \in$ $\overline{\mathcal{A}}^{y^{\prime}} \backslash\{\bar{\beta}\}=\mathcal{A} \backslash\{\bar{\alpha}\}$, and let $\beta \in \mathcal{M}$. Then (41), (42), (46) show that

$$
\begin{aligned}
\delta_{Q}^{|\beta|-|\alpha|} \mid & \partial^{\beta} \bar{P}_{\alpha}^{y^{\prime}}\left(y^{\prime}\right) \mid \leq \\
& \leq\left[\delta_{Q}^{|\beta|-|\alpha|}\left|\partial^{\beta} \stackrel{+}{P_{\alpha}^{y^{\prime}}}\left(y^{\prime}\right)\right|\right]+\left[\delta_{Q}^{|\bar{\beta}|-|\alpha|}\left|\partial^{\bar{\beta}} \stackrel{+}{P_{\alpha}^{y^{\prime}}}\left(y^{\prime}\right)\right|\right] \cdot\left[\delta_{Q}^{|\beta|-|\bar{\beta}|}\left|\partial^{\beta} \stackrel{+}{P_{\bar{\beta}}^{y^{\prime}}}\left(y^{\prime}\right)\right|\right] \\
& \leq\left[C \cdot\left(a_{1}\right)^{-(m+1)}\right]+\left[C \cdot\left(a_{1}\right)^{-(m+1)}\right] \cdot[1] \leq C^{\prime} \cdot\left(a_{1}\right)^{-(m+1)}
\end{aligned}
$$

Hence, conclusion (48) holds in all cases. 
Next, conclusion (49) holds for $\alpha=\bar{\beta}$, thanks to (43), (45), and (SU5). It remains to check conclusion (49) for $\alpha \in \overline{\mathcal{A}}^{y^{\prime}} \backslash\{\bar{\beta}\}=\mathcal{A} \backslash\{\bar{\alpha}\}$.

Suppose $\alpha \in \mathcal{A} \backslash\{\bar{\alpha}\}$, and let $S \subset E$, with $\#(S) \leq k_{\text {old }}^{\#}$. We apply (43) (for the given $\alpha$, and for $\bar{\beta}$ ), and we define

$$
\bar{\varphi}_{\alpha}^{S}=\stackrel{+}{\varphi}_{\alpha}^{S}-\left[\partial^{\bar{\beta}} \stackrel{+}{P}_{\alpha}^{y^{\prime}}\left(y^{\prime}\right)\right] \cdot \stackrel{+}{\varphi}_{\bar{\beta}}^{S} \in C_{\ell o c}^{m, \omega}\left(\mathbb{R}^{n}\right) .
$$

From (43)(a) and (41), we learn that whenever $|\beta|=m, x^{\prime}, x^{\prime \prime} \in \mathbb{R}^{n}$, $\left|x^{\prime}-x^{\prime \prime}\right| \leq 100 \delta_{Q}$, we have

$$
\begin{aligned}
& \left|\partial^{\beta} \bar{\varphi}_{\alpha}^{S}\left(x^{\prime}\right)-\partial^{\beta} \bar{\varphi}_{\alpha}^{S}\left(x^{\prime \prime}\right)\right| \leq \\
& \leq\left[C a_{2} \delta_{Q}^{|\alpha|-m} \frac{\omega\left(\left|x^{\prime}-x^{\prime \prime}\right|\right)}{\omega\left(\delta_{Q}\right)}\right]+\left[C\left(a_{1}\right)^{-(m+1)} \delta_{Q}^{|\alpha|-|\bar{\beta}|}\right]\left[C a_{2} \delta^{|\bar{\beta}|-m} \frac{\omega\left(\left|x^{\prime}-x^{\prime \prime}\right|\right)}{\omega\left(\delta_{Q}\right)}\right] \\
& \leq C^{\prime} \cdot\left(a_{1}\right)^{-(m+1)} \cdot a_{2} \cdot \delta_{Q}^{|\alpha|-m} \cdot \frac{\omega\left(\left|x^{\prime}-x^{\prime \prime}\right|\right)}{\omega\left(\delta_{Q}\right)}, \text { thanks to (SU5). }
\end{aligned}
$$

This proves conclusion (49)(a) for the given $\alpha$.

Also, for $x \in S$, we obtain from (41), (43)(b), (51) that

$$
\begin{aligned}
J_{x}\left(\bar{\varphi}_{\alpha}^{S}\right) & \in \frac{C \delta_{Q}^{|\alpha|-m}}{\omega\left(\delta_{Q}\right)} \sigma(x)+\left[C \cdot\left(a_{1}\right)^{-(m+1)} \delta_{Q}^{|\alpha|-|\bar{\beta}|}\right] \frac{C \delta^{|\bar{\beta}|-m}}{\omega\left(\delta_{Q}\right)} \sigma(x) \\
& \subseteq C^{\prime} \cdot\left(a_{1}\right)^{-(m+1)} \frac{\delta_{Q}^{|\alpha|-m}}{\omega\left(\delta_{Q}\right)} \sigma(x), \text { again thanks to (SU5) } .
\end{aligned}
$$

This proves conclusion (49)(b) for the given $\alpha$.

Finally, comparing (46) with (51), and applying (43)(c), we obtain conclusion (49)(c) for the given $\alpha$.

Thus, conclusion (49) holds also for $\alpha \in \mathcal{A} \backslash\{\bar{\alpha}\}$. The proof of Lemma 12.4 is complete

We are ready to give the main result of this section.

Lemma 12.5 The cube $Q^{+}$is $O K$.

Proof: For every $y^{\prime} \in\left(Q^{+}\right)^{* *}($ see $(1))$, we have constructed $\overline{\mathcal{A}}^{y^{\prime}}<\mathcal{A}$ (see (38)) and $\bar{P}_{\alpha}^{y^{\prime}} \in \mathcal{P}$ for $\alpha \in \overline{\mathcal{A}}^{y^{\prime}}$, satisfying (47), (48), (49).

We will check that the $\overline{\mathcal{A}}^{y^{\prime}}$ and $\bar{P}_{\alpha}^{y^{\prime}}$ satisfy $(\mathrm{OK} 1,2,3)$ for the cube $Q^{+}$.

In fact, (OK1) for $Q^{+}$is just (47).

Condition (OK2) for $Q^{+}$says that

$$
\delta_{Q^{+}}^{|\beta|-|\alpha|}\left|\partial^{\beta} \bar{P}_{\alpha}^{y^{\prime}}\left(y^{\prime}\right)\right| \leq\left(a_{1}\right)^{-(m+2)} \text { for all } \alpha \in \overline{\mathcal{A}}^{y^{\prime}}, \beta \in \mathcal{M} \text { with } \beta \geq \alpha .
$$

This estimate, without the restriction to $\beta \geq \alpha$, is immediate from (48) and (SU5), since $\delta_{Q^{+}}=2 \delta_{Q}$. 
Condition (OK3) for $Q^{+}$says that, given $\alpha \in \overline{\mathcal{A}}^{y^{\prime}}$ and $S \subset E$ with $\#(S) \leq k_{\text {old }}^{\#}$, there exists $\bar{\varphi}_{\alpha}^{S} \in C_{\text {loc }}^{m, \omega}\left(\mathbb{R}^{n}\right)$, with

$$
\begin{aligned}
\left|\partial^{\beta} \bar{\varphi}_{\alpha}^{S}\left(x^{\prime}\right)-\partial^{\beta} \bar{\varphi}_{\alpha}^{S}\left(x^{\prime \prime}\right)\right| & \leq\left(a_{1}\right)^{-(m+2)} \cdot \delta_{Q^{+}}^{|\alpha|-m-1} \cdot\left|x^{\prime}-x^{\prime \prime}\right|+ \\
& +\left(a_{1}\right)^{-(m+2)} \cdot a_{2} \cdot \delta_{Q^{+}}^{|\alpha|-m} \cdot \frac{\omega\left(\left|x^{\prime}-x^{\prime \prime}\right|\right)}{\omega\left(\delta_{Q^{+}}\right)}
\end{aligned}
$$

for $|\beta|=m, x^{\prime}, x^{\prime \prime} \in \mathbb{R}^{n},\left|x^{\prime}-x^{\prime \prime}\right| \leq \delta_{Q^{+}} ;$

$(52)(\mathrm{b}) \quad J_{x}\left(\bar{\varphi}_{\alpha}^{S}\right) \in\left(a_{1}\right)^{-(m+2)} \cdot \delta_{Q^{+}}^{|\alpha|-m} \cdot\left(\omega\left(\delta_{Q^{+}}\right)\right)^{-1} \cdot \sigma(x)$ for all $x \in S$; and

(52)(c) $J_{y^{\prime}}\left(\bar{\varphi}_{\alpha}^{S}\right)=\bar{P}_{\alpha}^{y^{\prime}}$.

We check that these conditions follow from (49). To do so, we recall that $\omega$ is a regular modulus of continuity, and that $\delta_{Q^{+}}=2 \delta_{Q} \leq 1$. Hence, $\omega\left(\delta_{Q}\right) \leq \omega\left(\delta_{Q^{+}}\right) \leq 2 \omega\left(\delta_{Q}\right)$.

In view of these remarks and (SU5), assertions (52)(a),(b),(c) are immediate from (49)(a),(b),(c), respectively.

Thus, conditions $(\mathrm{OK} 1,2,3)$ hold for the cube $Q^{+}$, the sets of multi-indices $\overline{\mathcal{A}}^{y^{\prime}}\left(y^{\prime} \in\left(Q^{+}\right)^{* *}\right)$, and the polynomials $\bar{P}_{\alpha}^{y^{\prime}}\left(\alpha \in \overline{\mathcal{A}}^{y^{\prime}}, y^{\prime} \in\left(Q^{+}\right)^{* *}\right)$.

This shows that the cube $Q^{+}$is OK. The proof of Lemma 12.5 is complete.

\section{Controlling Auxiliary Polynomials II}

In this section, we again place ourselves in the setting of Section 9, and we assume $(\mathrm{SU} 0, \ldots, 8)$. The result of this section is as follows.

Lemma 13.1 Fix an integer $k_{1}^{\#}$, satisfying

$$
k^{\#} \geq(D+1) \cdot k_{1}^{\#}, \quad k_{1}^{\#} \geq(D+1) \cdot k_{\text {old }}^{\#} .
$$

Suppose that

$$
Q \text { is a } \mathrm{CZ} \text { cube, }
$$

and

$$
y \in Q^{* * *} \text {. }
$$

Let $P_{\alpha}^{y} \in \mathcal{P}$ be a family of polynomials, indexed by $\alpha \in \mathcal{A}$, and assume that

$$
\text { Conditions } \left.(\mathrm{WL} 1)^{y},(\mathrm{WL} 2)^{y} \text {, (WL3) }\right)^{y} \text { hold for the } P_{\alpha}^{y} \text {. }
$$

(See Lemma 10.3.). Then we have the estimate

$$
\delta_{Q}^{|\beta|-|\alpha|}\left|\partial^{\beta} P_{\alpha}^{y}(y)\right| \leq\left(a_{1}\right)^{-(m+1)} \quad \text { for all } \alpha \in \mathcal{A}, \beta \in \mathcal{M} .
$$


Proof: Suppose (5) fails. There are finitely many dyadic cubes $\hat{Q}$ containing $Q$ (since, according to our definition, only subcubes of $Q^{0}$ are allowed as dyadic cubes). For such $\hat{Q}$, define

$$
\Phi(\hat{Q})=\max _{\substack{\beta \in \mathcal{M} \\ \alpha \in \mathcal{A}}} \delta_{\hat{Q}}^{|\beta|-|\alpha|}\left|\partial^{\beta} P_{\alpha}^{y}(y)\right| .
$$

Since (5) fails, we have $\Phi(Q)>\left(a_{1}\right)^{-(m+1)}$. Let $\bar{Q}$ be the maximal dyadic cube containing $Q$ with $\Phi(\bar{Q})>\left(a_{1}\right)^{-(m+1)}$. Thus,

$$
\begin{aligned}
& \Phi(\bar{Q})>\left(a_{1}\right)^{-(m+1)}, \\
& Q \subseteq \bar{Q}, \text { and } \\
& \text { either } \bar{Q}=Q^{0} \text {, or else } \Phi\left(\bar{Q}^{+}\right) \leq\left(a_{1}\right)^{-(m+1)} .
\end{aligned}
$$

We can easily check that $\bar{Q} \neq Q^{0}$. In fact, (11.3), (WL2)y and (SU5) show that

$$
\delta_{Q^{0}}^{|\beta|-|\alpha|}\left|\partial^{\beta} \bar{P}_{\alpha}^{y}(y)\right| \leq C \delta_{Q^{0}}^{|\beta|-|\alpha|} \leq C \delta_{Q^{0}}^{-m} \leq C^{\prime}\left(a_{1}\right)^{-m}<\left(a_{1}\right)^{-(m+1)}
$$

for all $\alpha \in \mathcal{A}, \beta \in \mathcal{M}$. Thus, $\Phi\left(Q^{0}\right)<\left(a_{1}\right)^{-(m+1)}$, and hence $\bar{Q} \neq Q^{0}$ by (7).

From (9) we now see that $\Phi\left(\bar{Q}^{+}\right) \leq\left(a_{1}\right)^{-(m+1)}$. A glance at the definition of $\Phi$ shows that $\Phi(\bar{Q})$ and $\Phi\left(\bar{Q}^{+}\right)$can differ at most by a factor of $2^{m}$. Therefore, $\Phi(\bar{Q}) \leq 2^{m} \cdot\left(a_{1}\right)^{-(m+1)}$.

Together with (6) and (7), this implies that

$$
\left(a_{1}\right)^{-(m+1)} \leq \max _{\substack{\beta \in \mathcal{M} \\ \alpha \in \mathcal{A}}} \delta_{\bar{Q}}^{|\beta|-|\alpha|}\left|\partial^{\beta} P_{\alpha}^{y}(y)\right| \leq 2^{m+1} \cdot\left(a_{1}\right)^{-(m+1)} .
$$

Note also that

$$
y \in \bar{Q}^{* * *}
$$

thanks to (3) and (8).

We prepare to apply the results of Section 12 to the cube $\bar{Q}$. Let us check that the assumptions (CAP1, .,5), made in that section, hold here for $\bar{Q}$. In fact, (CAP1) is merely our present hypothesis (1); (CAP2) for $\bar{Q}$ is our present observation (11); (CAP3) holds for $\bar{Q}$, since we showed above that $\bar{Q} \neq Q^{0}$; (CAP4) is our present hypothesis (4); and (CAP5) for $\bar{Q}$ is precisely our present result (10).

Hence, the results of Section 12 apply to the cube $\bar{Q}$. In particular, Lemma 12.5 shows that the cube $\bar{Q}^{+}$is OK. Consequently, $\bar{Q}^{+}$is almost OK. On the other hand, (8) shows that $\bar{Q}^{+}$properly contains the CZ cube $Q$. Hence, by definition of a $\mathrm{CZ}$ cube, the cube $\bar{Q}^{+}$cannot be almost OK. This contradiction proves that (5) cannot fail.

The proof of Lemma 13.1 is complete. 


\section{Controlling the Main Polynomials}

In this section, we again place ourselves in the setting of Section 9, and we assume (SU0 , . , 8). Our goal is to control the polynomials in $\mathcal{K}_{f}^{\#}\left(y, k_{1}^{\#}, M\right)$ in terms of the $\mathrm{CZ}$ cubes $Q$, for suitable $k_{1}^{\#}$ and $M$.

Lemma 14.1 Let $Q, Q^{\prime}$ be $\mathrm{CZ}$ cubes that abut or coincide. Suppose we are given

$$
y \in Q^{* * *}, \quad y^{\prime} \in\left(Q^{\prime}\right)^{* * *}
$$

and

$$
P \in \mathcal{K}_{f}^{\#}\left(y, k_{1}^{\#}, C\right)
$$

with

$$
k^{\#} \geq(D+1) \cdot k_{1}^{\#}, \quad k_{1}^{\#} \geq(D+1) \cdot k_{2}^{\#}, \quad \text { and } \quad k_{2}^{\#} \geq k_{\text {old }}^{\#} .
$$

Then there exists

$$
P^{\prime} \in \mathcal{K}_{f}^{\#}\left(y^{\prime}, k_{2}^{\#}, C^{\prime}\right)
$$

with

(5) $\left|\partial^{\beta}\left(P^{\prime}-P\right)\left(y^{\prime}\right)\right| \leq C^{\prime \prime} \cdot\left(a_{1}\right)^{-(m+1)} \cdot \omega\left(\delta_{Q}\right) \cdot \delta_{Q}^{m-|\beta|} \quad$ for all $\beta \in \mathcal{M}$.

Proof: By Lemma 10.2, there exists

$$
\tilde{P} \in \mathcal{K}_{f}\left(y^{\prime}, k_{2}^{\#}, C\right),
$$

with

$$
\left|\partial^{\beta}(\tilde{P}-P)\left(y^{\prime}\right)\right| \leq C^{\prime} \omega\left(\delta_{Q}\right) \cdot \delta_{Q}^{m-|\beta|} \quad \text { for } \beta \in \mathcal{M} .
$$

(Here, we use the fact that $\left|y-y^{\prime}\right| \leq C^{\prime} \delta_{Q}$. This follows from (1) and Lemma 11.2, since $Q$ and $Q^{\prime}$ are $\mathrm{CZ}$ cubes that abut or coincide.

Note also that $\omega\left(\left|y-y^{\prime}\right|\right) \leq C^{\prime} \omega\left(\delta_{Q}\right)$, since $\omega$ is a regular modulus of continuity. We note also the fact that $y \in Q^{* * *} \subseteq\left(Q^{0}\right)^{* * *} \subset B\left(y^{0}, a_{1}\right)$ by (11.2), and similarly $y^{\prime} \in\left(Q^{\prime}\right)^{* * *} \subseteq\left(Q^{0}\right)^{* * *} \subset B\left(y^{0}, a_{1}\right)$.)

From (6) and the definition of $\mathcal{K}_{f}$, we have the following.

(8) Given $S \subset E$ with $\#(S) \leq k_{2}^{\#}$, there exists $\tilde{F}^{S} \in C^{m, \omega}\left(\mathbb{R}^{n}\right)$, with

$$
\left\|\tilde{F}^{S}\right\|_{C^{m, \omega}\left(\mathbb{R}^{n}\right)} \leq C, J_{x}\left(\tilde{F}^{S}\right) \in f(x)+C \sigma(x)
$$

for all $x \in S$ and $J_{y^{\prime}}\left(\tilde{F}^{S}\right)=\tilde{P}$. 
In particular, taking $S=$ empty set in (8), we learn that

$$
\left|\partial^{\beta} \tilde{P}\left(y^{\prime}\right)\right| \leq C \quad \text { for all } \beta \in \mathcal{M} \text {. }
$$

Also, (2) and the definition of $\mathcal{K}_{f}^{\#}$ give $\partial^{\beta} P(y)=0$ for all $\beta \in \mathcal{A}$.

Applying (SU0), we see that $\partial^{\gamma+\beta} P(y)=0$ for all $\beta \in \mathcal{A},|\gamma| \leq m-|\beta|$.

Since $\partial^{\beta} P$ is a polynomial of degree at most $m-|\beta|$, it follows that $\partial^{\beta} P$ is the zero polynomial, for all $\beta \in \mathcal{A}$. Hence, (7) implies

$$
\left|\partial^{\beta} \tilde{P}\left(y^{\prime}\right)\right| \leq C^{\prime} \omega\left(\delta_{Q}\right) \cdot \delta_{Q}^{m-|\beta|} \text { for all } \beta \in \mathcal{A} .
$$

Next, since $y^{\prime} \in B\left(y^{0}, a_{1}\right)$ as noted above, Lemma 10.3 applies, with $y^{\prime}$ in place of $y$. Thus, we obtain polynomials $P_{\alpha}^{y^{\prime}} \in \mathcal{P}(\alpha \in \mathcal{A})$, satisfying conditions $(\mathrm{WL} 1)^{y^{\prime}},(\mathrm{WL} 2)^{y^{\prime}}$, (WL3) $)^{y^{\prime}}$.

We now check that the hypotheses of Lemma 13.1 hold here, with our present $Q^{\prime}, y^{\prime}, P_{\alpha}^{y^{\prime}}(\alpha \in \mathcal{A})$ in place of $Q, y, P_{\alpha}^{y}(\alpha \in \mathcal{A})$.

In fact, hypothesis (1) of Lemma 13.1 is immediate from our present hypothesis (3); and hypothesis (2) of Lemma 13.1 (with $Q^{\prime}$ in place of $Q$ ) is a hypothesis of the Lemma we are now proving. Hypothesis (3) of Lemma 13.1 (with $Q^{\prime}, y^{\prime}$ in place of $Q, y$ ) is contained in our present hypothesis (1). Finally, hypothesis (4) of Lemma 13.1 (with $Q^{\prime}, y^{\prime}, P_{\alpha}^{y^{\prime}}$ in place of $Q, y, P_{\alpha}^{y}$ ) says that $(\mathrm{WL} 1)^{y^{\prime}}$, (WL2) $)^{y^{\prime}}$, (WL3) $)^{y^{\prime}}$ hold for the $P_{\alpha}^{y^{\prime}}(\alpha \in \mathcal{A})$; this is precisely the defining property of the $P_{\alpha}^{y^{\prime}}$. Thus, as claimed, the hypotheses of Lemma 13.1 hold for $Q^{\prime}, y^{\prime},\left(P_{\alpha}^{y^{\prime}}\right)_{\alpha \in \mathcal{A}}$. Applying that lemma, we conclude that

$$
\delta_{Q^{\prime}}^{|\beta|-|\alpha|}\left|\partial^{\beta} P_{\alpha}^{y^{\prime}}\left(y^{\prime}\right)\right| \leq\left(a_{1}\right)^{-(m+1)} \quad \text { for all } \alpha \in \mathcal{A}, \beta \in \mathcal{M} .
$$

We now define

$$
P^{\prime}=\tilde{P}-\sum_{\alpha \in \mathcal{A}}\left[\partial^{\alpha} \tilde{P}\left(y^{\prime}\right)\right] \cdot P_{\alpha}^{y^{\prime}} \in \mathcal{P} .
$$

Note that

$$
\partial^{\beta} P^{\prime}\left(y^{\prime}\right)=\partial^{\beta} \tilde{P}\left(y^{\prime}\right)-\sum_{\alpha \in \mathcal{A}}\left[\partial^{\alpha} \tilde{P}\left(y^{\prime}\right)\right] \cdot \partial^{\beta} P_{\alpha}^{y^{\prime}}\left(y^{\prime}\right)=0 \quad \text { for all } \beta \in \mathcal{A},
$$

thanks to $(\mathrm{WL} 1)^{y^{\prime}}$.

Note also that, for any $\alpha \in \mathcal{A}$ and $\beta \in \mathcal{M}$, we have

$$
\begin{aligned}
\left|\partial^{\beta}\left\{\left[\partial^{\alpha} \tilde{P}\left(y^{\prime}\right)\right] \cdot P_{\alpha}^{y^{\prime}}\right\}\left(y^{\prime}\right)\right| & =\left|\partial^{\alpha} \tilde{P}\left(y^{\prime}\right)\right| \cdot\left|\partial^{\beta} P_{\alpha}^{y^{\prime}}\left(y^{\prime}\right)\right| \\
& \leq\left[C^{\prime} \omega\left(\delta_{Q}\right) \cdot \delta_{Q}^{m-|\alpha|}\right] \cdot\left[\left(a_{1}\right)^{-(m+1)} \delta_{Q^{\prime}}^{|\alpha|-|\beta|}\right] \\
& \leq C^{\prime \prime}\left(a_{1}\right)^{-(m+1)} \omega\left(\delta_{Q}\right) \cdot \delta_{Q}^{m-|\beta|}
\end{aligned}
$$


thanks to (10), (11), and Lemma 11.2. Hence (12) shows that

$$
\left|\partial^{\beta}\left(P^{\prime}-\tilde{P}\right)\left(y^{\prime}\right)\right| \leq C^{\prime \prime}\left(a_{1}\right)^{-(m+1)} \omega\left(\delta_{Q}\right) \cdot \delta_{Q}^{m-|\beta|} \quad \text { for all } \beta \in \mathcal{M} .
$$

Together with (7) and (SU5), (14) implies

$$
\left|\partial^{\beta}\left(P^{\prime}-P\right)\left(y^{\prime}\right)\right| \leq C^{\prime \prime} \cdot\left(a_{1}\right)^{-(m+1)} \omega\left(\delta_{Q}\right) \delta_{Q}^{m-|\beta|} \text { for all } \beta \in \mathcal{M},
$$

which is conclusion (5). Moreover, suppose $S \subset E$ with $\#(S) \leq k_{2}^{\#}$ (and hence also $\#(S) \leq k_{1}^{\#}$; see $\left.(3)\right)$.

Let $\tilde{F}^{S}$ be as in (8), and, for each $\alpha \in \mathcal{A}$, let $\varphi_{\alpha}^{S} \in C_{\text {loc }}^{m, \omega}\left(\mathbb{R}^{n}\right)$ be as in (WL3) $)^{y^{\prime}}$. We introduce a cutoff function $\theta$ on $\mathbb{R}^{n}$, with

$$
\|\theta\|_{C^{m+1}\left(\mathbb{R}^{n}\right)} \leq C^{\prime}, \quad \theta=1 \text { on } B\left(y^{\prime}, 1 / 20\right), \quad \operatorname{supp} \theta \subset B\left(y^{\prime}, 1 / 10\right),
$$

and we define

$$
F^{S}=\tilde{F}^{S}-\sum_{\alpha \in \mathcal{A}}\left[\partial^{\alpha} \tilde{P}\left(y^{\prime}\right)\right] \theta \varphi_{\alpha}^{S}
$$

Thus, $F^{S} \in C^{m, \omega}\left(\mathbb{R}^{m}\right)$, since $\tilde{F}^{S} \in C^{m, \omega}\left(\mathbb{R}^{n}\right), \varphi_{\alpha}^{S} \in C_{\text {loc }}^{m, \omega}\left(\mathbb{R}^{n}\right), \theta \in C^{m+1}\left(\mathbb{R}^{n}\right)$, and supp $\theta \subset B\left(y^{\prime}, 1 / 10\right)$. We prepare to estimate the $C^{m, \omega}$-norm of $F^{S}$.

From (WL2) $)^{y^{\prime}},(\mathrm{WL} 3)^{y^{\prime}}(\mathrm{c})$, and (SU5), we have

$$
\left|\partial^{\beta} \varphi_{\alpha}^{S}\left(y^{\prime}\right)\right| \leq C^{\prime} \quad \text { for }|\beta| \leq m, \alpha \in \mathcal{A}
$$

Hence, (WL3) $)^{y^{\prime}}$ (a) shows that

$$
\left|\partial^{\beta} \varphi_{\alpha}^{S}\left(x^{\prime}\right)\right| \leq C^{\prime} \quad \text { for }|\beta|=m, \alpha \in \mathcal{A}, x^{\prime} \in B\left(y^{\prime}, 1\right) .
$$

From (17) and (18), we obtain

$$
\left|\partial^{\beta} \varphi_{\alpha}^{S}\right| \leq C^{\prime} \quad \text { on } B\left(y^{\prime}, 1\right) \text {, for }|\beta| \leq m, \alpha \in \mathcal{A} .
$$

From (15), (19), and (WL3) $)^{\prime}(\mathrm{a})$, together with (SU7), we obtain

$$
\left\|\theta \varphi_{\alpha}^{S}\right\|_{C^{m, \omega}\left(\mathbb{R}^{n}\right)} \leq C^{\prime} \text { for all } \alpha \in \mathcal{A} .
$$

Together with (9), this yields

$$
\left\|\left[\partial^{\alpha} \tilde{P}\left(y^{\prime}\right)\right] \cdot \theta \varphi_{\alpha}^{S}\right\|_{C^{m, \omega}\left(\mathbb{R}^{n}\right)} \leq C^{\prime} \quad \text { for all } \alpha \in \mathcal{A} .
$$

Putting (8) and (20) into (16), we learn that

$$
\left\|F^{S}\right\|_{C^{m, \omega}\left(\mathbb{R}^{n}\right)} \leq C^{\prime} .
$$


Next, suppose $x \in S \cap B\left(y^{\prime}, 1\right)$. Then from (WL3) $)^{y^{\prime}}(\mathrm{b})$ and (19), we have (22)

$$
J_{x}\left(c_{1} \varphi_{\alpha}^{S}\right) \in \sigma(x) \text { and }\left|\partial^{\beta}\left[J_{x}\left(c_{1} \varphi_{\alpha}^{S}\right)\right](x)\right| \leq 1 \text { for }|\beta| \leq m, \alpha \in \mathcal{A},
$$

for a small enough controlled constant $c_{1}>0$.

From (15), we have also

$$
\left|\partial^{\beta}\left[J_{x}\left(c_{2} \theta\right)\right](x)\right| \leq 1 \quad \text { for }|\beta| \leq m,
$$

for a small enough controlled constant $c_{2}>0$.

From (22), (23), and the Whitney $\omega$-convexity assumption (SU2), we see that

$$
J_{x}\left(\theta \varphi_{\alpha}^{S}\right) \in C^{\prime} \sigma(x) \text { for all } \alpha \in \mathcal{A} .
$$

(Here, we take $\delta=1$ in the definition of Whitney $\omega$-convexity.)

We have proven (24) for $x \in S \cap B\left(y^{\prime}, 1\right)$, but of course it holds also for $x \in S, x \notin B\left(y^{\prime}, 1\right)$, since then (15) gives $J_{x}\left(\theta \varphi_{\alpha}^{S}\right)=0$.

Thus, (24) holds for all $x \in S$. From (9), (24) we obtain

$$
J_{x}\left(\sum_{\alpha \in \mathcal{A}}\left[\partial^{\alpha} \tilde{P}\left(y^{\prime}\right)\right] \cdot \theta \varphi_{\alpha}^{S}\right) \in C^{\prime} \sigma(x) \text { for all } x \in S .
$$

Also, from (8), we have

$$
J_{x}\left(\tilde{F}^{S}\right) \in f(x)+C \sigma(x) \text { for all } x \in S .
$$

Putting (25), (26) into (16), we find that

$$
J_{x}\left(F^{S}\right) \in f(x)+C^{\prime} \sigma(x) \text { for all } x \in S .
$$

Moreover, (8), (12), (15), (16), and (WL3) $)^{y^{\prime}}(\mathrm{c})$ show that

$$
\begin{aligned}
J_{y^{\prime}}\left(F^{S}\right) & =J_{y^{\prime}}\left(\tilde{F}^{S}\right)-\sum_{\alpha \in \mathcal{A}}\left[\partial^{\alpha} \tilde{P}\left(y^{\prime}\right)\right] J_{y^{\prime}}\left(\theta \varphi_{\alpha}^{S}\right) \\
& =J_{y^{\prime}}\left(\tilde{F}^{S}\right)-\sum_{\alpha \in \mathcal{A}}\left[\partial^{\alpha} \tilde{P}\left(y^{\prime}\right)\right] J_{y^{\prime}}\left(\varphi_{\alpha}^{S}\right) \\
& =\tilde{P}-\sum_{\alpha \in \mathcal{A}}\left[\partial^{\alpha} \tilde{P}\left(y^{\prime}\right)\right] P_{\alpha}^{y^{\prime}}=P^{\prime} .
\end{aligned}
$$

In view of $(21),(27),(28)$, we have proven the following:

Given $S \subset E$ with $\#(S) \leq k_{2}^{\#}$, there exists $F^{S} \in C^{m, \omega}\left(\mathbb{R}^{n}\right)$, with $\left\|F^{S}\right\|_{C^{m, \omega}\left(\mathbb{R}^{n}\right)} \leq C^{\prime}, J_{x}\left(F^{S}\right) \in f(x)+C^{\prime} \sigma(x)$ for all $x \in S$, and $J_{y^{\prime}}\left(F^{S}\right)=P^{\prime}$.

By definition, this means that $P^{\prime} \in \mathcal{K}_{f}\left(y^{\prime}, k_{2}^{\#}, C^{\prime}\right)$. Since also $P^{\prime}$ satisfies (13), we have

$$
P^{\prime} \in \mathcal{K}_{f}^{\#}\left(y^{\prime}, k_{2}^{\#}, C^{\prime}\right),
$$

which is conclusion (4). Thus, we have proven that $P^{\prime} \in \mathcal{P}$ satisfies (4) and (5). The proof of Lemma 14.1 is complete. 
Lemma 14.2 Fix $k_{1}^{\#}$, with

$$
k^{\#} \geq(D+1) \cdot k_{1}^{\#} \quad \text { and } \quad k_{1}^{\#} \geq(D+1) \cdot k_{\text {old }}^{\#} \cdot
$$

Suppose that

$$
\begin{aligned}
& Q \text { is a } C Z \text { cube, } \\
& y \in Q^{* *}, \quad \text { and } \\
& P_{1}, P_{2} \in \mathcal{K}_{f}^{\#}\left(y, k_{1}^{\#}, C\right) .
\end{aligned}
$$

Then

$$
\left|\partial^{\beta}\left(P_{1}-P_{2}\right)(y)\right| \leq\left(a_{1}\right)^{-(m+1)} \cdot a_{2}^{-1} \cdot \omega\left(\delta_{Q}\right) \cdot \delta_{Q}^{m-|\beta|} \quad \text { for }|\beta| \leq m .
$$

Proof: Suppose (33) fails. Under this assumption, we will show that

$$
Q \text { is a proper subcube of } Q^{0} \text {, and }
$$$$
Q^{+} \text {is } \mathrm{OK} \text {. }
$$

This will lead to a contradiction, since $Q^{+}$is a dyadic cube that properly contains a $\mathrm{CZ}$ cube, and therefore $Q^{+}$cannot be almost OK.

Consequently, the proof of Lemma 14.2 is reduced to showing (34) and (35) under the assumption that (33) fails.

In view of (32), we know that

(36) $\partial^{\beta} P_{1}(y)=\partial^{\beta} P_{2}(y)=0$ for all $\beta \in \mathcal{A}$, and

(37) Given $S \subset E$ with $\#(S) \leq k_{1}^{\#}$, there exist $F_{1}^{S}, F_{2}^{S} \in C^{m, \omega}\left(\mathbb{R}^{n}\right)$, with $\left\|F_{i}^{S}\right\|_{C^{m, \omega}\left(\mathbb{R}^{n}\right)} \leq C, J_{x}\left(F_{i}^{S}\right) \in f(x)+C \sigma(x)$ for all $x \in S$, and $J_{y}\left(F_{i}^{S}\right)=$ $P_{i}(i=1,2)$.

In particular, taking $S=$ empty set in (37), we find that

$$
\left|\partial^{\beta} P_{1}(y)\right|,\left|\partial^{\beta} P_{2}(y)\right| \leq C \quad \text { for }|\beta| \leq m .
$$

It is now easy to check (34). Since $Q$ is dyadic, it is enough to show that $Q \neq Q^{0}$.

We have $c a_{1} \leq \delta_{Q^{0}} \leq a_{1}$ (see (11.3)), hence also $\omega\left(\delta_{Q^{0}}\right) \geq \omega\left(c a_{1}\right) \geq c a_{1}$ since $\omega$ is a regular modulus of continuity. Hence, for $|\beta| \leq m$, we have

$$
\left(a_{1}\right)^{-(m+1)} \cdot a_{2}^{-1} \cdot \omega\left(\delta_{Q^{0}}\right) \cdot \delta_{Q^{0}}^{m-|\beta|} \geq c^{\prime} \cdot\left(a_{1}\right)^{-(m+1)} \cdot a_{2}^{-1} \cdot a_{1} \cdot a_{1}^{m}=c^{\prime} a_{2}^{-1}
$$

Also, for $|\beta| \leq m,(38)$ gives

$$
\left|\partial^{\beta}\left(P_{1}-P_{2}\right)(y)\right| \leq C^{\prime} .
$$


From (39), (40) and (SU7), we have

$$
\left|\partial^{\beta}\left(P_{1}-P_{2}\right)(y)\right| \leq\left(a_{1}\right)^{-(m+1)} a_{2}^{-1} \omega\left(\delta_{Q^{0}}\right) \delta_{Q^{0}}^{m-|\beta|} \text { for }|\beta| \leq m .
$$

On the other hand, we are assuming that (33) fails. Hence, $Q \neq Q^{0}$, proving (34).

We start the proof of (35). We are assuming that (33) fails. Let

$$
y^{\prime} \in\left(Q^{+}\right)^{* *}
$$

be given.

Then $y, y^{\prime} \in Q^{* * *}$, and $P_{1}, P_{2} \in \mathcal{K}_{f}^{\#}\left(y, k_{1}^{\#}, C\right)$. Also, $k^{\#} \geq(D+1) \cdot k_{1}^{\#}$ and $k_{1}^{\#} \geq(D+1) \cdot k_{\text {old }}^{\#}$. Applying Lemma 14.1, with $k_{2}^{\#}=k_{\text {old }}^{\#}$, we obtain polynomials

$$
\tilde{P}_{1}, \tilde{P}_{2} \in \mathcal{K}_{f}^{\#}\left(y^{\prime}, k_{\text {old }}^{\#}, C^{\prime}\right)
$$

with

$$
\left|\partial^{\beta}\left(\tilde{P}_{i}-P_{i}\right)\left(y^{\prime}\right)\right| \leq C^{\prime \prime} \cdot\left(a_{1}\right)^{-(m+1)} \cdot \omega\left(\delta_{Q}\right) \cdot \delta_{Q}^{m-|\beta|} \quad \text { for }|\beta| \leq m .
$$

From (43), we see that

$$
\begin{aligned}
\max _{\beta \in \mathcal{M}} & {\left[\omega\left(\delta_{Q}\right) \delta_{Q}^{m-|\beta|}\right]^{-1} \cdot\left|\partial^{\beta}\left(P_{1}-P_{2}\right)\left(y^{\prime}\right)\right| \leq } \\
& \leq 2 C \cdot\left(a_{1}\right)^{-(m+1)}+\max _{\beta \in \mathcal{M}}\left[\omega\left(\delta_{Q}\right) \delta_{Q}^{m-|\beta|}\right]^{-1} \cdot\left|\partial^{\beta}\left(\tilde{P}_{1}-\tilde{P}_{2}\right)\left(y^{\prime}\right)\right| .
\end{aligned}
$$

Also, for $\beta \in \mathcal{M}$ we have

$$
\begin{aligned}
\left|\partial^{\beta}\left(P_{1}-P_{2}\right)(y)\right| & =\left|\sum_{|\gamma| \leq m-|\beta|} \frac{1}{\gamma !}\left(\partial^{\gamma+\beta}\left(P_{1}-P_{2}\right)\left(y^{\prime}\right)\right) \cdot\left(y-y^{\prime}\right)^{\gamma}\right| \\
& \leq C \max _{|\gamma| \leq m-|\beta|} \delta_{Q}^{|\gamma|} \cdot\left|\partial^{\gamma+\beta}\left(P_{1}-P_{2}\right)\left(y^{\prime}\right)\right| \quad\left(\text { since } y, y^{\prime} \in Q^{* * *}\right) \\
& \leq C \delta_{Q}^{-|\beta|} \cdot \max _{\beta^{\prime} \in \mathcal{M}} \delta^{\left|\beta^{\prime}\right|} \cdot\left|\partial^{\beta^{\prime}}\left(P_{1}-P_{2}\right)\left(y^{\prime}\right)\right|,
\end{aligned}
$$

and therefore,

$$
\begin{aligned}
& \max _{\beta \in \mathcal{M}}\left[\omega\left(\delta_{Q}\right) \cdot \delta_{Q}^{m-|\beta|}\right]^{-1} \cdot\left|\partial^{\beta}\left(P_{1}-P_{2}\right)(y)\right| \leq \\
& \leq C \cdot \max _{\beta \in \mathcal{M}}\left[\omega\left(\delta_{Q}\right) \delta_{Q}^{m-|\beta|}\right]^{-1} \cdot\left|\partial^{\beta}\left(P_{1}-P_{2}\right)\left(y^{\prime}\right)\right|
\end{aligned}
$$

Also, since we are assuming that (33) fails, we have

$$
\left(a_{1}\right)^{-(m+1)} \cdot a_{2}^{-1}<\max _{\beta \in \mathcal{M}}\left[\omega\left(\delta_{Q}\right) \delta_{Q}^{m-|\beta|}\right]^{-1} \cdot\left|\partial^{\beta}\left(P_{1}-P_{2}\right)(y)\right| .
$$


Combining (44), (45), (46), we learn that $\left(a_{1}\right)^{-(m+1)} \cdot a_{2}^{-1} \leq C^{\prime} \cdot\left(a_{1}\right)^{-(m+1)}+C^{\prime} \max _{\beta \in \mathcal{M}}\left[\omega\left(\delta_{Q}\right) \delta_{Q}^{m-|\beta|}\right]^{-1} \cdot\left|\partial^{\beta}\left(\tilde{P}_{1}-\tilde{P}_{2}\right)\left(y^{\prime}\right)\right|$

Consequently, by (SU7), we have

$$
\max _{\beta \in \mathcal{M}}\left[\omega\left(\delta_{Q}\right) \cdot \delta_{Q}^{m-|\beta|}\right]^{-1} \cdot\left|\partial^{\beta}\left(\tilde{P}_{1}-\tilde{P}_{2}\right)\left(y^{\prime}\right)\right| \geq c^{\prime} \cdot\left(a_{1}\right)^{-(m+1)} \cdot a_{2}^{-1} .
$$

From (42) and the definition of $\mathcal{K}_{f}^{\#}$, we have

$$
\partial^{\beta} \tilde{P}_{1}\left(y^{\prime}\right)=\partial^{\beta} \tilde{P}_{2}\left(y^{\prime}\right)=0 \quad \text { for } \beta \in \mathcal{A}
$$

and also

(49) Given $S \subset E$ with $\#(S) \leq k_{\text {old }}^{\#}$, there exist $\tilde{F}_{1}^{S}, \tilde{F}_{2}^{S} \in C^{m, \omega}\left(\mathbb{R}^{n}\right)$, with

$$
\begin{aligned}
& \left\|\tilde{F}_{i}^{S}\right\|_{C^{m, \omega}\left(\mathbb{R}^{n}\right)} \leq C^{\prime}, J_{x}\left(\tilde{F}_{i}^{S}\right) \in f(x)+C^{\prime} \sigma(x) \text { for all } x \in S, \\
& J_{y^{\prime}}\left(\tilde{F}_{i}^{S}\right)=\tilde{P}_{i}(i=1,2) .
\end{aligned}
$$

Immediately from (49), we obtain

(50) Given $S \subset E$ with $\#(S) \leq k_{\text {old }}^{\#}$, there exists $\tilde{F}^{S} \in C^{m, \omega}\left(\mathbb{R}^{n}\right)$, with

$$
\begin{aligned}
& \left\|\tilde{F}^{S}\right\|_{C^{m, \omega}\left(\mathbb{R}^{n}\right)} \leq C^{\prime}, J_{x}\left(\tilde{F}^{S}\right) \in C^{\prime} \sigma(x) \text { for all } x \in S, \text { and } \\
& J_{y^{\prime}}\left(\tilde{F}^{S}\right)=\tilde{P}_{1}-\tilde{P}_{2} .
\end{aligned}
$$

Now pick $\bar{\beta} \in \mathcal{M}$ to maximize $\left[\omega\left(\delta_{Q}\right) \cdot \delta_{Q}^{m-|\bar{\beta}|}\right]^{-1} \cdot\left|\partial^{\bar{\beta}}\left(\tilde{P}_{1}-\tilde{P}_{2}\right)\left(y^{\prime}\right)\right|$, and define

$$
\Omega=\partial^{\bar{\beta}}\left(\tilde{P}_{1}-\tilde{P}_{2}\right)\left(y^{\prime}\right) .
$$

By definition, and by (47), we have

$$
\left|\partial^{\beta}\left(\tilde{P}_{1}-\tilde{P}_{2}\right)\left(y^{\prime}\right)\right| \leq|\Omega| \cdot \delta_{Q}^{|\bar{\beta}|-|\beta|} \quad \text { for all } \beta \in \mathcal{M},
$$

and

$$
|\Omega| \geq c \cdot\left(a_{1}\right)^{-(m+1)} \cdot a_{2}^{-1} \cdot \omega\left(\delta_{Q}\right) \cdot \delta_{Q}^{m-|\bar{\beta}|} .
$$

In particular, $\Omega \neq 0$. We define

$$
\bar{P}=\left(\tilde{P}_{1}-\tilde{P}_{2}\right) / \Omega \in \mathcal{P} .
$$


From (51), (52) we have

$$
\left|\partial^{\beta} \bar{P}\left(y^{\prime}\right)\right| \leq \delta_{Q}^{|\bar{\beta}|-|\beta|} \quad \text { for all } \beta \in \mathcal{M},
$$

and

$$
\partial^{\bar{\beta}} \bar{P}\left(y^{\prime}\right)=1 \text {. }
$$

From (48) we have

$$
\partial^{\beta} \bar{P}\left(y^{\prime}\right)=0 \quad \text { for all } \beta \in \mathcal{A} .
$$

Comparing (56) to (57), we see that

$$
\bar{\beta} \notin \mathcal{A} \text {. }
$$

Also, from (50), (53), (54), we learn the following.

(59) Given $S \subset E$ with $\#(S) \leq k_{\text {old }}^{\#}$, there exists $\bar{F}^{S} \in C^{m, \omega}\left(\mathbb{R}^{n}\right)$, with
(a) $\left\|\bar{F}^{S}\right\|_{C^{m, \omega}\left(\mathbb{R}^{n}\right)} \leq \frac{C^{\prime}}{|\Omega|} \leq C^{\prime \prime} \cdot\left(a_{1}\right)^{m+1} \cdot a_{2} \cdot\left[\omega\left(\delta_{Q}\right) \cdot \delta_{Q}^{m-|\bar{\beta}|}\right]^{-1}$;
(b) $J_{x}\left(\bar{F}^{S}\right) \in \frac{C^{\prime}}{|\Omega|} \sigma(x) \subseteq C^{\prime \prime} \cdot\left(a_{1}\right)^{m+1} \cdot a_{2} \cdot\left[\omega\left(\delta_{Q}\right) \cdot \delta_{Q}^{m-|\beta|}\right]^{-1} \sigma(x)$ for all $x \in S$; and
(c) $J_{y^{\prime}}\left(\bar{F}^{S}\right)=\bar{P}$.

In view of (59)(a), the function $\bar{F}^{S}$ in (59) satisfies

(60) $\left|\partial^{\beta} \bar{F}^{S}\left(x^{\prime}\right)-\partial^{\beta} \bar{F}^{S}\left(x^{\prime \prime}\right)\right| \leq C^{\prime \prime} \cdot\left(a_{1}\right)^{m+1} \cdot a_{2} \cdot \delta_{Q}^{|\bar{\beta}|-m} \cdot \frac{\omega\left(\left|x^{\prime}-x^{\prime \prime}\right|\right)}{\omega\left(\delta_{Q}\right)}$ for $|\beta|=m$, $x^{\prime}, x^{\prime \prime} \in \mathbb{R}^{n},\left|x^{\prime}-x^{\prime \prime}\right| \leq 1$.

Recall that $y^{\prime} \in\left(Q^{+}\right)^{* *} \subset Q^{* * *} \subset\left(Q^{0}\right)^{* * *} \subset B\left(y^{0}, a_{1}\right)$ (see 11.2).

Hence, Lemma 10.3 shows that there exist polynomials $P_{\alpha}^{y^{\prime}}(\alpha \in \mathcal{A})$, for which

$$
(\mathrm{WL} 1)^{y^{\prime}},(\mathrm{WL} 2)^{y^{\prime}},(\mathrm{WL} 3)^{y^{\prime}} \text { hold for the } P_{\alpha}^{y^{\prime}}(\alpha \in \mathcal{A}) \text {. }
$$

We now define

$$
\begin{aligned}
& \overline{\mathcal{A}}^{y^{\prime}}=\mathcal{A} \cup\{\bar{\beta}\}, \\
& \bar{P}_{\bar{\beta}}=\bar{P}, \quad \text { and } \\
& \bar{P}_{\alpha}=P_{\alpha}^{y^{\prime}}-\left[\partial^{\bar{\beta}} P_{\alpha}^{y^{\prime}}\left(y^{\prime}\right)\right] \cdot \bar{P} \quad \text { for all } \alpha \in \mathcal{A} .
\end{aligned}
$$

Thus, we have defined $\bar{P}_{\beta} \in \mathcal{P}$ for all $\beta \in \overline{\mathcal{A}}^{y^{\prime}}$. Note that (63) and (64) do not conflict, and moreover $\mathcal{A}$ is a proper subset of $\overline{\mathcal{A}}^{y^{\prime}}$, thanks to (58). Hence, Lemma 3.2 shows that

$$
\overline{\mathcal{A}}^{y^{\prime}}<\mathcal{A} .
$$


We will check that

$$
\partial^{\beta} \bar{P}_{\alpha}\left(y^{\prime}\right)=\delta_{\beta \alpha} \quad \text { for all } \beta, \alpha \in \overline{\mathcal{A}}^{y^{\prime}}
$$

In fact, (66) holds for $\alpha=\bar{\beta}$, thanks to (56), (57), (63).

For $\alpha, \beta \in \mathcal{A}$, we have

$$
\partial^{\beta} \bar{P}_{\alpha}\left(y^{\prime}\right)=\partial^{\beta} P_{\alpha}^{y^{\prime}}\left(y^{\prime}\right)-\left[\partial^{\bar{\beta}} P_{\alpha}^{y^{\prime}}\left(y^{\prime}\right)\right] \cdot \partial^{\beta} \bar{P}\left(y^{\prime}\right)=\delta_{\beta \alpha},
$$

by (WL1) $y^{y^{\prime}}$ and (57). Hence, (66) holds for $\alpha, \beta \in \mathcal{A}$.

Finally, for $\alpha \in \mathcal{A}, \beta=\bar{\beta}$, we have

$$
\partial^{\beta} \bar{P}_{\alpha}\left(y^{\prime}\right)=\partial^{\bar{\beta}} P_{\alpha}^{y^{\prime}}\left(y^{\prime}\right)-\left[\partial^{\bar{\beta}} P_{\alpha}^{y^{\prime}}\left(y^{\prime}\right)\right] \cdot \partial^{\bar{\beta}} \bar{P}\left(y^{\prime}\right)=0,
$$

thanks to (56). Hence, (66) holds also for $\alpha \in \mathcal{A}, \beta=\bar{\beta}$.

Thus, (66) holds in all cases.

Next, we apply Lemma 13.1, with $y^{\prime}$ and $P_{\alpha}^{y^{\prime}}$ in place of $y$ and $P_{\alpha}^{y}$. We check that the hypotheses of that lemma are satisfied. In fact, we have $k^{\#} \geq(D+1) \cdot k_{1}^{\#}$ and $k_{1}^{\#} \geq(D+1) \cdot k_{\text {old }}^{\#}$, by our present hypothesis $(29)$. Also, $Q$ is a CZ cube, by our present hypothesis (30).

We have $y^{\prime} \in Q^{* * *}$, thanks to (41).

Finally, (WL1) $)^{y^{\prime}},(\mathrm{WL} 2)^{y^{\prime}},(\mathrm{WL} 3)^{y^{\prime}}$ hold for the $P_{\alpha}^{y^{\prime}}(\alpha \in \mathcal{A})$; see (61).

Thus, as claimed, the hypotheses of Lemma 13.1 are satisfied, with our present $y^{\prime}$ and $P_{\alpha}^{y^{\prime}}$ in place of $y, P_{\alpha}^{y}$. Applying that lemma, we learn that

$$
\delta_{Q}^{|\beta|-|\alpha|}\left|\partial^{\beta} P_{\alpha}^{y^{\prime}}\left(y^{\prime}\right)\right| \leq\left(a_{1}\right)^{-(m+1)} \quad \text { for all } \alpha \in \mathcal{A}, \beta \in \mathcal{M} .
$$

Using (67), we can check that

$$
\left|\partial^{\beta} \bar{P}_{\alpha}\left(y^{\prime}\right)\right| \leq C \cdot\left(a_{1}\right)^{-(m+1)} \cdot \delta_{Q}^{|\alpha|-|\beta|} \text { for all } \alpha \in \overline{\mathcal{A}}^{y^{\prime}}, \beta \in \mathcal{M} .
$$

In fact, for $\alpha=\bar{\beta}$, (68) is immediate from (55), (63), and (SU5).

For $\alpha \in \mathcal{A}, \beta \in \mathcal{M}$, we learn from (55), (64), (67) that

$$
\begin{aligned}
\left|\partial^{\beta} \bar{P}_{\alpha}\left(y^{\prime}\right)\right| & \leq\left|\partial^{\beta} P_{\alpha}^{y^{\prime}}\left(y^{\prime}\right)\right|+\left|\partial^{\bar{\beta}} P_{\alpha}^{y^{\prime}}\left(y^{\prime}\right)\right| \cdot\left|\partial^{\beta} \bar{P}\left(y^{\prime}\right)\right| \\
& \leq\left[\left(a_{1}\right)^{-(m+1)} \delta_{Q}^{|\alpha|-|\beta|}\right]+\left[\left(a_{1}\right)^{-(m+1)} \delta_{Q}^{|\alpha|-|\bar{\beta}|}\right] \cdot\left[\delta_{Q}^{|\bar{\beta}|-|\beta|}\right] \\
& \leq C \cdot\left(a_{1}\right)^{-(m+1)} \cdot \delta_{Q}^{|\alpha|-|\beta|}, \quad \text { so again (68) holds. }
\end{aligned}
$$

Thus (68) holds in both cases. 
Let $S \subset E$ be given, with $\#(S) \leq k_{\text {old }}^{\#}$. Let $\bar{F}^{S} \in C^{m, \omega}\left(\mathbb{R}^{n}\right)$ be as in (59), and, for $\alpha \in \mathcal{A}$, let $\varphi_{\alpha}^{S} \in C_{\text {loc }}^{m, \omega}\left(\mathbb{R}^{n}\right)$ be as in (WL3) $)^{y^{\prime}}$. (Note that (WL3) y $^{\prime}$ applies, since $k_{1}^{\#} \geq k_{\text {old }}^{\#}$.) We define

$$
\bar{\varphi} \bar{\beta}=\bar{F}^{S}
$$

and

$$
\bar{\varphi}_{\alpha}^{S}=\varphi_{\alpha}^{S}-\left[\partial^{\bar{\beta}} P_{\alpha}^{y^{\prime}}\left(y^{\prime}\right)\right] \cdot \bar{F}^{S} \quad \text { for all } \alpha \in \mathcal{A} .
$$

Thus $\bar{\varphi}_{\alpha}^{S} \in C_{\ell o c}^{m, \omega}\left(\mathbb{R}^{n}\right)$ for all $\alpha \in \overline{\mathcal{A}}^{y^{\prime}}$.

We will check that

$$
\left|\partial^{\beta} \bar{\varphi}_{\alpha}^{S}\left(x^{\prime}\right)-\partial^{\beta} \bar{\varphi}_{\alpha}^{S}\left(x^{\prime \prime}\right)\right| \leq C a_{2} \cdot \delta_{Q}^{|\alpha|-m} \cdot \frac{\omega\left(\left|x^{\prime}-x^{\prime \prime}\right|\right)}{\omega\left(\delta_{Q}\right)}
$$

for $\alpha \in \overline{\mathcal{A}}^{y^{\prime}},|\beta|=m, x^{\prime}, x^{\prime \prime} \in \mathbb{R}^{n},\left|x^{\prime}-x^{\prime \prime}\right| \leq 50 \delta_{Q}$.

In fact, for $\alpha=\bar{\beta},(71)$ is immediate from (60), (69), and (SU5).

Suppose $\alpha \in \mathcal{A}$. Then, for $|\beta|=m, x^{\prime}, x^{\prime \prime} \in \mathbb{R}^{n},\left|x^{\prime}-x^{\prime \prime}\right| \leq 1$, we have

$$
\begin{aligned}
\mid & \partial^{\beta} \bar{\varphi}_{\alpha}^{S}\left(x^{\prime}\right)-\partial^{\beta} \bar{\varphi}_{\alpha}^{S}\left(x^{\prime \prime}\right) \mid \leq \\
\leq & \left|\partial^{\beta} \varphi_{\alpha}^{S}\left(x^{\prime}\right)-\partial^{\beta} \varphi_{\alpha}^{S}\left(x^{\prime \prime}\right)\right|+\left|\partial^{\bar{\beta}} P_{\alpha}^{y^{\prime}}\left(y^{\prime}\right)\right| \cdot\left|\partial^{\beta} \bar{F}^{S}\left(x^{\prime}\right)-\partial^{\beta} \bar{F}^{S}\left(x^{\prime \prime}\right)\right| \\
\leq & {\left[C a_{2} \omega\left(\left|x^{\prime}-x^{\prime \prime}\right|\right)\right]+\left[C a_{1}^{-(m+1)} \delta_{Q}^{|\alpha|-|\bar{\beta}|}\right]\left[C^{\prime \prime}\left(a_{1}\right)^{m+1} a_{2} \delta_{Q}^{|\bar{\beta}|-m} \frac{\omega\left(\left|x^{\prime}-x^{\prime \prime}\right|\right)}{\omega\left(\delta_{Q}\right)}\right] } \\
& \quad\left(\text { see }(\mathrm{WL} 3)^{y^{\prime}}(\mathrm{a}),(67),(59)\right) \\
= & C a_{2} \omega\left(\left|x^{\prime}-x^{\prime \prime}\right|\right)+C a_{2} \delta_{Q}^{|\alpha|-m} \frac{\omega\left(\left|x^{\prime}-x^{\prime \prime}\right|\right)}{\omega\left(\delta_{Q}\right)} \leq C^{\prime} a_{2} \delta_{Q}^{|\alpha|-m} \frac{\omega\left(\left|x^{\prime}-x^{\prime \prime}\right|\right)}{\omega\left(\delta_{Q}\right)},
\end{aligned}
$$

since $|\alpha| \leq m$ and $\delta_{Q}, \omega\left(\delta_{Q}\right) \leq 1$. So, again (71) holds.

Thus, (71) holds in all cases.

Next, we check that

$$
J_{x}\left(\bar{\varphi}_{\alpha}^{S}\right) \in C \delta_{Q}^{|\alpha|-m}\left(\omega\left(\delta_{Q}\right)\right)^{-1} \sigma(x) \quad \text { for all } x \in S, \alpha \in \overline{\mathcal{A}}^{y^{\prime}} .
$$

In fact, for $\alpha=\bar{\beta},(72)$ is immediate from (59)(b), (69), (SU5) and (SU7).

Suppose $\alpha \in \mathcal{A}$. Then, for $x \in S$, we have

$$
\begin{aligned}
& J_{x}\left(\bar{\varphi}_{\alpha}^{S}\right)=J_{x}\left(\varphi_{\alpha}^{S}\right)-\left[\partial^{\bar{\beta}} P_{\alpha}^{y^{\prime}}\left(y^{\prime}\right)\right] \cdot J_{x}\left(\bar{F}^{S}\right) \quad(\text { see }(70)) \\
& \in[C \sigma(x)]+\left|\partial^{\bar{\beta}} P_{\alpha}^{y^{\prime}}\left(y^{\prime}\right)\right| \cdot\left[C^{\prime \prime} \cdot\left(a_{1}\right)^{m+1} \cdot a_{2} \cdot\left(\omega\left(\delta_{Q}\right)\right)^{-1} \cdot \delta_{Q}^{|\bar{\beta}|-m} \sigma(x)\right] \\
& \quad \quad\left(\text { see }(\text { WL3 } 3)^{y^{\prime}}(\mathrm{b}) \text { and }(59)(\mathrm{b})\right) \\
& \subset[C \sigma(x)]+\left[a_{1}^{-(m+1)} \cdot \delta_{Q}^{|\alpha|-|\bar{\beta}|}\right] \cdot\left[C^{\prime \prime} a_{1}^{(m+1)} \cdot a_{2} \cdot \delta_{Q}^{|\bar{\beta}|-m} \cdot\left(\omega\left(\delta_{Q}\right)\right)^{-1} \sigma(x)\right] \\
& \quad(\operatorname{see}(67)) \\
& \subset C \sigma(x)+C^{\prime \prime} a_{2} \delta_{Q}^{|\alpha|-m}\left(\omega\left(\delta_{Q}\right)\right)^{-1} \sigma(x) \subset C^{\prime} \delta_{Q}^{|\alpha|-m}\left(\omega\left(\delta_{Q}\right)\right)^{-1} \sigma(x),
\end{aligned}
$$


since $\delta_{Q}, \omega\left(\delta_{Q}\right) \leq 1,|\alpha| \leq m$, and $a_{2}<1$ (see (SU7)). Hence, again (72) holds.

Thus, (72) holds in all cases. We also check that

$$
J_{y^{\prime}}\left(\bar{\varphi}_{\alpha}^{S}\right)=\bar{P}_{\alpha} \quad \text { for all } \alpha \in \overline{\mathcal{A}}^{y^{\prime}} .
$$

In fact, for $\alpha=\bar{\beta}$, (73) is immediate from (59)(c), (63), (69).

Suppose $\alpha \in \mathcal{A}$. Then

$$
\begin{aligned}
J_{y^{\prime}}\left(\bar{\varphi}_{\alpha}^{S}\right) & =J_{y^{\prime}}\left(\varphi_{\alpha}^{S}\right)-\left[\partial^{\bar{\beta}} P_{\alpha}^{y^{\prime}}\left(y^{\prime}\right)\right] \cdot J_{y^{\prime}}\left(\bar{F}^{S}\right) \quad(\text { see }(70)) \\
& =P_{\alpha}^{y^{\prime}}-\left[\partial^{\bar{\beta}} P_{\alpha}^{y^{\prime}}\left(y^{\prime}\right)\right] \cdot \bar{P} \quad\left(\text { see }(\text { WL3 } 3)^{y^{\prime}}(\mathrm{c}) \text { and }(59)(\mathrm{c})\right) \\
& =\bar{P}_{\alpha} \quad(\text { see }(64)) .
\end{aligned}
$$

So, again (73) holds. Thus, (73) holds in all cases.

Given $y^{\prime} \in\left(Q^{+}\right)^{* *}($ see $(41))$, we have constructed $\overline{\mathcal{A}}^{y^{\prime}}<\mathcal{A}$ (see (65)), along with $\bar{P}_{\alpha} \in \mathcal{P}\left(\alpha \in \overline{\mathcal{A}}^{y^{\prime}}\right)$ (see (63), (64)), satisfying (66) and (68). Moreover, given $\alpha \in \overline{\mathcal{A}}^{y^{\prime}}$ and $S \subset E$ with $\#(S) \leq k_{\text {old }}^{\#}$, we have exhibited $\bar{\varphi}_{\alpha}^{S} \in C_{\ell o c}^{m, \omega}\left(\mathbb{R}^{n}\right)$, satisfying (71), (72), (73).

We will now check that $\overline{\mathcal{A}}^{y^{\prime}}$ and $\bar{P}_{\alpha}\left(\alpha \in \overline{\mathcal{A}}^{y^{\prime}}\right)$ satisfy conditions (OK1,2,3) for the cube $Q^{+}$.

In fact, (OK1) for $\overline{\mathcal{A}}^{y^{\prime}}, \bar{P}_{\alpha}, Q^{+}$says simply that $\partial^{\beta} \bar{P}_{\alpha}\left(y^{\prime}\right)=\delta_{\beta \alpha}$ for $\beta, \alpha \in \overline{\mathcal{A}}^{y^{\prime}}$, which is precisely (66).

Condition (OK2) for $\overline{\mathcal{A}}^{y^{\prime}}, \bar{P}_{\alpha}, Q^{+}$says that

$$
\left(\delta_{Q^{+}}\right)^{|\beta|-|\alpha|}\left|\partial^{\beta} \bar{P}_{\alpha}\left(y^{\prime}\right)\right| \leq\left(a_{1}\right)^{-(m+2)} \quad \text { for all } \alpha \in \overline{\mathcal{A}}^{y^{\prime}}, \beta \in \mathcal{M} \text { with } \beta \geq \alpha .
$$

This assertion, without the restriction to $\beta \geq \alpha$, is immediate from (68) and (SU5), since $\delta_{Q^{+}}=2 \delta_{Q}$.

Condition (OK3) for $\overline{\mathcal{A}}^{y^{\prime}}, \bar{P}_{\alpha}, Q^{+}$says that

(74) given $\alpha \in \overline{\mathcal{A}}^{y^{\prime}}$ and $S \subset E$ with $\#(S) \leq k_{\text {old }}^{\#}$, there exists $\bar{\varphi}_{\alpha}^{S} \in$ $C_{\ell o c}^{m, \omega}\left(\mathbb{R}^{n}\right)$, with

(a) $\left|\partial^{\beta} \bar{\varphi}_{\alpha}^{S}\left(x^{\prime}\right)-\partial^{\beta} \bar{\varphi}_{\alpha}^{S}\left(x^{\prime \prime}\right)\right| \leq a_{1}^{-(m+2)} \delta_{Q^{+}}^{|\alpha|-m-1} \cdot\left|x^{\prime}-x^{\prime \prime}\right|+\left(a_{1}\right)^{-(m+2)}$. $a_{2} \cdot \delta_{Q^{+}}^{|\alpha|-m}$.

. $\frac{\omega\left(\left|x^{\prime}-x^{\prime \prime}\right|\right)}{\omega\left(\delta_{Q^{+}}\right)}$for $|\beta|=m, x^{\prime}, x^{\prime \prime} \in \mathbb{R}^{n},\left|x^{\prime}-x^{\prime \prime}\right| \leq \delta_{Q^{+}} ;$

(b) $J_{x}\left(\bar{\varphi}_{\alpha}^{S}\right) \in\left(a_{1}\right)^{-(m+2)} \delta_{Q^{+}}^{|\alpha|-m}\left(\omega\left(\delta_{Q^{+}}\right)\right)^{-1} \cdot \sigma(x)$ for all $x \in S$; and

(c) $J_{y^{\prime}}\left(\bar{\varphi}_{\alpha}^{S}\right)=\bar{P}_{\alpha}$.

We use the $\bar{\varphi}_{\alpha}^{S} \in C_{\ell o c}^{m, \omega}\left(\mathbb{R}^{n}\right)$ that satisfy (71), (72), (73). 
Since $\delta_{Q^{+}}=2 \delta_{Q}$ and $\omega$ is a regular modulus of continuity, we have

$$
\omega\left(\delta_{Q}\right) \leq \omega\left(\delta_{Q^{+}}\right) \leq 2 \omega\left(\delta_{Q}\right) .
$$

Hence, (74)(a) is immediate from (71) and (SU5); and (74)(b) is immediate from (72) and (SU5).

Also, (74)(c) is precisely (73).

Thus, as claimed, (OK1,2,3) hold for $Q^{+}, \overline{\mathcal{A}}^{y^{\prime}}, \bar{P}_{\alpha}\left(\alpha \in \overline{\mathcal{A}}^{y^{\prime}}\right)$, for any given $y^{\prime} \in\left(Q^{+}\right)^{* *}$. By definition, this tells us that $Q^{+}$is OK.

The proof of (35) is complete.

Hence, also, the proof of Lemma 14.2 is complete.

Lemma 14.3 Suppose $y \in Q^{* *}$ and $y^{\prime} \in\left(Q^{\prime}\right)^{* *}$, where $Q$ and $Q^{\prime}$ are $C Z$ cubes that abut. Let $P \in \mathcal{K}_{f}^{\#}\left(y, k_{A}^{\#}, C\right)$ and $P^{\prime} \in \mathcal{K}_{f}^{\#}\left(y^{\prime}, k_{A}^{\#}, C\right)$ be given, where

$$
k^{\#} \geq(D+1) \cdot k_{A}^{\#} \quad \text { and } \quad k_{A}^{\#} \geq(D+1)^{2} \cdot k_{\text {old }}^{\#} .
$$

Then we have

$$
\left|\partial^{\beta}\left(P^{\prime}-P\right)\left(y^{\prime}\right)\right| \leq C^{\prime} \cdot\left(a_{1}\right)^{-(m+1)} \cdot\left(a_{2}\right)^{-1} \cdot \omega\left(\delta_{Q}\right) \cdot \delta_{Q}^{m-|\beta|} \quad \text { for }|\beta| \leq m .
$$

Proof: Let $k_{B}^{\#}=(D+1) \cdot k_{\text {old }}^{\#}$. Then, by Lemma 14.1, there exists $\tilde{P} \in$ $\mathcal{K}_{f}^{\#}\left(y^{\prime}, k_{B}^{\#}, C^{\prime}\right)$, with

$$
\left|\partial^{\beta}(\tilde{P}-P)\left(y^{\prime}\right)\right| \leq C^{\prime \prime} \cdot\left(a_{1}\right)^{-(m+1)} \cdot \omega\left(\delta_{Q}\right) \cdot \delta_{Q}^{m-|\beta|} \text { for }|\beta| \leq m .
$$

In particular, both $\tilde{P}$ and $P^{\prime}$ belong to $\mathcal{K}_{f}^{\#}\left(y^{\prime}, k_{B}^{\#}, C^{\prime \prime \prime}\right)$, with $y^{\prime} \in\left(Q^{\prime}\right)^{* *}$.

Thus, Lemma 14.2 shows that

$$
\left|\partial^{\beta}\left(P^{\prime}-\tilde{P}\right)\left(y^{\prime}\right)\right| \leq\left(a_{1}\right)^{-(m+1)} \cdot\left(a_{2}\right)^{-1} \cdot \omega\left(\delta_{Q^{\prime}}\right) \delta_{Q^{\prime}}^{m-|\beta|} \quad \text { for }|\beta| \leq m .
$$

By Lemma 11.2, we have

$$
\frac{1}{2} \delta_{Q} \leq \delta_{Q^{\prime}} \leq 2 \delta_{Q}
$$

Since $\omega$ is a regular modulus of continuity, it follows that

$$
\frac{1}{2} \omega\left(\delta_{Q}\right) \leq \omega\left(\delta_{Q^{\prime}}\right) \leq 2 \omega\left(\delta_{Q}\right)
$$

Putting these remarks into (78), we find that

$$
\left|\partial^{\beta}\left(P^{\prime}-\tilde{P}\right)\left(y^{\prime}\right)\right| \leq \tilde{C} \cdot\left(a_{1}\right)^{-(m+1)} \cdot\left(a_{2}\right)^{-1} \cdot \omega\left(\delta_{Q}\right) \cdot \delta_{Q}^{m-|\beta|} \quad \text { for }|\beta| \leq m .
$$

Adding (77) and (79), and recalling (SU7), we obtain the conclusion (76) of Lemma 14.3. The proof of the lemma is complete. 
We shall need analogues of Lemmas 14.1 and 14.3 in which the cubes $Q, Q^{\prime}$ need not abut.

Lemma 14.4 Let $Q, Q^{\prime}$ be distinct $C Z$ cubes, with centers $y, y^{\prime}$ respectively. Let

$$
P \in \mathcal{K}_{f}^{\#}\left(y, k_{1}^{\#}, C\right)
$$

with

$$
k^{\#} \geq(D+1) \cdot k_{1}^{\#}, k_{1}^{\#} \geq(D+1) \cdot k_{2}^{\#} \quad \text { and } \quad k_{2}^{\#} \geq k_{\text {old }}^{\#} .
$$

Then there exists

$$
P^{\prime} \in \mathcal{K}_{f}^{\#}\left(y^{\prime}, k_{2}^{\#}, C^{\prime}\right)
$$

with

$$
\left|\partial^{\beta}\left(P^{\prime}-P\right)\left(y^{\prime}\right)\right| \leq C^{\prime \prime} \cdot\left(a_{1}\right)^{-(m+1)} \cdot \omega\left(\left|y-y^{\prime}\right|\right) \cdot\left|y-y^{\prime}\right|^{m-|\beta|}
$$

for $|\beta| \leq m$.

Proof: We have $y, y^{\prime} \in Q^{0} \subset B\left(y^{0}, a_{1}\right)$, hence $\left|y-y^{\prime}\right| \leq 2 a_{1} \leq 1$.

Hence, Lemma 10.2 shows that there exists

$$
\tilde{P} \in \mathcal{K}_{f}\left(y^{\prime}, k_{2}^{\#}, C\right),
$$

with

$$
\left|\partial^{\beta}(\tilde{P}-P)\left(y^{\prime}\right)\right| \leq C^{\prime} \omega\left(\left|y-y^{\prime}\right|\right) \cdot\left|y-y^{\prime}\right|^{m-|\beta|} \quad \text { for }|\beta| \leq m .
$$

From (84), we have

(86) Given $S \subset E$ with $\#(S) \leq k_{2}^{\#}$, there exists $\tilde{F}^{S} \in C^{m, \omega}\left(\mathbb{R}^{n}\right)$, with $\left\|\tilde{F}^{S}\right\|_{C^{m, \omega}\left(\mathbb{R}^{n}\right)} \leq C, J_{x}\left(\tilde{F}^{S}\right) \in f(x)+C \sigma(x)$ for all $x \in S, J_{y^{\prime}}\left(\tilde{F}^{S}\right)=\tilde{P}$.

In particular, taking $S=$ empty set in (86), we obtain

$$
\left|\partial^{\beta} \tilde{P}\left(y^{\prime}\right)\right| \leq C \text { for }|\beta| \leq m .
$$

Also, (80) gives $\partial^{\beta} P(y)=0$ for all $\beta \in \mathcal{A}$. Hence, by (SU0), we have also $\partial^{\gamma+\beta} P(y)=0$ for all $\beta \in \mathcal{A},|\gamma| \leq m-|\beta|$. Since $\partial^{\beta} P$ is a polynomial of degree of most $m-|\beta|$, it follows that $\partial^{\beta} P$ is the zero polynomial, for all $\beta \in \mathcal{A}$. Hence, (85) implies

$$
\left|\partial^{\beta} \tilde{P}\left(y^{\prime}\right)\right| \leq C^{\prime} \omega\left(\left|y-y^{\prime}\right|\right) \cdot\left|y-y^{\prime}\right|^{m-|\beta|} \quad \text { for } \beta \in \mathcal{A} .
$$

Next, since $y^{\prime} \in B\left(y^{0}, a_{1}\right)$, Lemma 10.3 applies, with $y^{\prime}$ in place of $y$. 
Let $P_{\alpha}^{y^{\prime}} \in \mathcal{P}(\alpha \in \mathcal{A})$ satisfy $(\mathrm{WL} 1)^{y^{\prime}},(\mathrm{WL} 2)^{y^{\prime}},(\mathrm{WL} 3)^{y^{\prime}}$. From (WL2) $)^{y^{\prime}}$ and (SU5), we have

$$
\left|\partial^{\beta} P_{\alpha}^{y^{\prime}}\left(y^{\prime}\right)\right| \leq C^{\prime} \quad \text { for } \alpha \in \mathcal{A},|\beta| \leq m
$$

We next check that the hypotheses of Lemma 13.1 hold for the cube $Q^{\prime}$, the point $y^{\prime}$, and the polynomials $P_{\alpha}^{y^{\prime}}(\alpha \in \mathcal{A})$.

In fact, (81) shows that $k^{\#} \geq(D+1) \cdot k_{1}^{\#} \geq(D+1) \cdot k_{\text {old }}^{\#}$.

We are assuming in Lemma 14.4 that $Q^{\prime}$ is a CZ cube, and that $y^{\prime}$ is the center of $Q^{\prime}$, hence $y^{\prime} \in\left(Q^{\prime}\right)^{* * *}$. The defining property of the $P_{\alpha}^{y^{\prime}} \in \mathcal{P}$ is that they satisfy (WL1) $)^{y^{\prime}}, \ldots,(\text { WL3) })^{y^{\prime}}$. Thus, as claimed, the hypotheses of Lemma 13.1 hold for $Q^{\prime}, y^{\prime},\left(P_{\alpha}^{y^{\prime}}\right)_{\alpha \in \mathcal{A}}$. Applying that lemma, we learn that

$$
\delta_{Q^{\prime}}^{|\beta|-|\alpha|}\left|\partial^{\beta} P_{\alpha}^{y^{\prime}}\left(y^{\prime}\right)\right| \leq\left(a_{1}\right)^{-(m+1)} \quad \text { for all } \alpha \in \mathcal{A},|\beta| \leq m \text {. }
$$

Now define

$$
P^{\prime}=\tilde{P}-\sum_{\alpha \in \mathcal{A}}\left[\partial^{\alpha} \tilde{P}\left(y^{\prime}\right)\right] \cdot P_{\alpha}^{y^{\prime}} \in \mathcal{P}
$$

Note that

$$
\partial^{\beta} P^{\prime}\left(y^{\prime}\right)=\partial^{\beta} \tilde{P}\left(y^{\prime}\right)-\sum_{\alpha \in \mathcal{A}}\left[\partial^{\alpha} \tilde{P}\left(y^{\prime}\right)\right] \cdot \partial^{\beta} P_{\alpha}^{y^{\prime}}\left(y^{\prime}\right)=0 \quad \text { for } \beta \in \mathcal{A},
$$

thanks to (WL1) $)^{y^{\prime}}$.

We check that $P^{\prime} \in \mathcal{K}_{f}\left(y^{\prime}, k_{2}^{\#}, C^{\prime}\right)$. In fact, let $S \subset E$, with $\#(S) \leq k_{2}^{\#}$.

Then also $\#(S) \leq k_{1}^{\#}$. Let $\tilde{F}^{S} \in C^{m, \omega}\left(\mathbb{R}^{n}\right)$ be as in (86), and, for each $\alpha \in \mathcal{A}$, let $\varphi_{\alpha}^{S} \in C_{\text {loc }}^{m, \omega}\left(\mathbb{R}^{n}\right)$ be as in (WL3) $)^{y^{\prime}}$.

We introduce a cutoff function $\theta$ on $\mathbb{R}^{n}$, satisfying

$$
\|\theta\|_{C^{m+1}\left(\mathbb{R}^{n}\right)} \leq C^{\prime}, \theta=1 \text { on } B\left(y^{\prime}, 1 / 20\right), \operatorname{supp} \theta \subset B\left(y^{\prime}, 1 / 10\right) .
$$

We then define

$$
F^{S}=\tilde{F}^{S}-\sum_{\alpha \in \mathcal{A}}\left[\partial^{\alpha} \tilde{P}\left(y^{\prime}\right)\right] \cdot \theta \varphi_{\alpha}^{S} \quad \text { on } \mathbb{R}^{n}
$$

Note that $F^{S} \in C^{m, \omega}\left(\mathbb{R}^{n}\right)$, since $\tilde{F}^{S} \in C^{m \omega}\left(\mathbb{R}^{n}\right), \varphi_{\alpha}^{S} \in C_{\ell o c}^{m, \omega}\left(\mathbb{R}^{n}\right), \theta \in$ $C^{m+1}\left(\mathbb{R}^{n}\right)$, and $\operatorname{supp} \theta \subset B\left(y^{\prime}, 1 / 10\right)$. Let us estimate the derivatives of $F^{S}$.

From (WL2) $)^{y^{\prime}},(\mathrm{WL} 3)^{y^{\prime}}(\mathrm{c})$, and (SU5), we have

$$
\left|\partial^{\beta} \varphi_{\alpha}^{S}\left(y^{\prime}\right)\right| \leq C^{\prime} \quad \text { for } \alpha \in \mathcal{A},|\beta| \leq m
$$


Hence, (WL3) $)^{y^{\prime}}(\mathrm{a})$ and (SU7) show that

$$
\left|\partial^{\beta} \varphi_{\alpha}^{S}\right| \leq C^{\prime} \text { on } B\left(y^{\prime}, 1\right) \text {, for } \alpha \in \mathcal{A},|\beta|=m .
$$

(Here, we use the fact that $\omega$ is a regular modulus of continuity, hence $\omega(t) \leq 1$ for $0 \leq t \leq 1$.)

From (95), (96), it follows that

$$
\left|\partial^{\beta} \varphi_{\alpha}^{S}\right| \leq C^{\prime} \quad \text { on } B\left(y^{\prime}, 1\right) \text {, for } \alpha \in \mathcal{A},|\beta| \leq m \text {. }
$$

Again using (WL3) $)^{\prime}($ a) and (SU7), we see that

$$
\left|\partial^{\beta} \varphi_{\alpha}^{S}\left(x^{\prime}\right)-\partial^{\beta} \varphi_{\alpha}^{S}\left(x^{\prime \prime}\right)\right| \leq \omega\left(\left|x^{\prime}-x^{\prime \prime}\right|\right)
$$

for $\alpha \in \mathcal{A},|\beta|=m, x^{\prime}, x^{\prime \prime} \in \mathbb{R}^{n},\left|x^{\prime}-x^{\prime \prime}\right| \leq 1$.

From (93), (97), (98), we conclude that

$$
\left\|\varphi_{\alpha}^{S} \theta\right\|_{C^{m, \omega}\left(\mathbb{R}^{n}\right)} \leq C^{\prime} \text { for all } \alpha \in \mathcal{A} .
$$

Putting (86), (87), (99) into (94), we see that

$$
\left\|F^{S}\right\|_{C^{m, \omega}\left(\mathbb{R}^{n}\right)} \leq C^{\prime} .
$$

Next, suppose $\alpha \in \mathcal{A}$ and $x \in S \cap B\left(y^{\prime}, 1\right)$. Then $J_{x}\left(\varphi_{\alpha}^{S}\right) \in C \sigma(x)$ by (WL3) $y^{y^{\prime}}$ (b) and also $\left|\partial^{\beta}\left(J_{x}\left(\varphi_{\alpha}^{S}\right)\right)(x)\right| \leq C^{\prime}$ for $|\beta| \leq m$, by (97). Moreover, we have $\left|\partial^{\beta}\left(J_{x}(\theta)\right)(x)\right| \leq C^{\prime}$ for $|\beta| \leq m$, by (93). Hence, our Whitney $\omega$-convexity assumption (SU2) shows that

$$
J_{x}\left(\theta \varphi_{\alpha}^{S}\right) \in C^{\prime} \sigma(x) .
$$

(Here, we take $\delta=1$ in the definition of Whitney $\omega$-convexity.)

We have proven (101) for $\alpha \in \mathcal{A}, x \in S \cap B\left(y^{\prime}, 1\right)$.

However, for $\alpha \in \mathcal{A}, x \in S \backslash B\left(y^{\prime}, 1\right)$, (101) holds trivially, since then $J_{x}\left(\theta \varphi_{\alpha}^{S}\right)=0$ by (93). Thus (101) holds for all $\alpha \in \mathcal{A}, x \in S$.

Putting (86), (87), (101) into (94), we find that

$$
J_{x}\left(F^{S}\right) \in J_{x}\left(\tilde{F}^{S}\right)+C^{\prime} \sigma(x) \subset f(x)+C^{\prime \prime} \sigma(x) \text { for all } x \in S .
$$

Next, note that

$$
\begin{aligned}
J_{y^{\prime}}\left(F^{S}\right) & =J_{y^{\prime}}\left(\tilde{F}^{S}\right)-\sum_{\alpha \in \mathcal{A}}\left[\partial^{\alpha} \tilde{P}\left(y^{\prime}\right)\right] \cdot J_{y^{\prime}}\left(\theta \varphi_{\alpha}^{S}\right) \quad(\text { see }(94)) \\
& =J_{y^{\prime}}\left(\tilde{F}^{S}\right)-\sum_{\alpha \in \mathcal{A}}\left[\partial^{\alpha} \tilde{P}\left(y^{\prime}\right)\right] \cdot J_{y^{\prime}}\left(\varphi_{\alpha}^{S}\right) \quad(\text { see }(93)) \\
& =\tilde{P}-\sum_{\alpha \in \mathcal{A}}\left[\partial^{\alpha} \tilde{P}\left(y^{\prime}\right)\right] \cdot P_{\alpha}^{y^{\prime}} \quad\left(\text { see }(86) \text { and }(\text { WL } 3)^{y^{\prime}}(\mathrm{c})\right) \\
& =P^{\prime} \quad(\text { see }(91))
\end{aligned}
$$


Thus, given $S \subset E$ with $\#(S) \leq k_{2}^{\#}$, we have exhibited $F^{S} \in C^{m, \omega}\left(\mathbb{R}^{n}\right)$, satisfying (100), (102), (103). By definition, we therefore have

$$
P^{\prime} \in \mathcal{K}_{f}\left(y^{\prime}, k_{2}^{\#}, C^{\prime}\right) \text {. }
$$

Since also (92) holds, we conclude that

$$
P^{\prime} \in \mathcal{K}_{f}^{\#}\left(y^{\prime}, k_{2}^{\#}, C^{\prime}\right) .
$$

Next, we estimate the derivatives of $P^{\prime}-P$ at $y^{\prime}$. From (19) we have

$$
\left|\partial^{\beta}\left(P^{\prime}-\tilde{P}\right)\left(y^{\prime}\right)\right| \leq \sum_{\alpha \in \mathcal{A}}\left|\partial^{\alpha} \tilde{P}\left(y^{\prime}\right)\right| \cdot\left|\partial^{\beta} P_{\alpha}^{y^{\prime}}\left(y^{\prime}\right)\right|, \quad \text { for }|\beta| \leq m .
$$

If $|\beta| \geq|\alpha|, \alpha \in \mathcal{A}$, then (88) and (89) yield

$$
\begin{aligned}
\left|\partial^{\alpha} \tilde{P}\left(y^{\prime}\right)\right| \cdot\left|\partial^{\beta} P_{\alpha}^{y^{\prime}}\left(y^{\prime}\right)\right| & \leq C^{\prime} \omega\left(\left|y-y^{\prime}\right|\right) \cdot\left|y-y^{\prime}\right|^{m-|\alpha|} \\
& \leq C^{\prime} \omega\left(\left|y-y^{\prime}\right|\right) \cdot\left|y-y^{\prime}\right|^{m-|\beta|} .
\end{aligned}
$$

If instead $|\beta| \leq|\alpha|, \alpha \in \mathcal{A}$, then (88) and (90) imply (107) $\left|\partial^{\alpha} \tilde{P}\left(y^{\prime}\right)\right| \cdot\left|\partial^{\beta} P_{\alpha}^{y^{\prime}}\left(y^{\prime}\right)\right| \leq C^{\prime} \omega\left(\left|y-y^{\prime}\right|\right) \cdot\left|y-y^{\prime}\right|^{m-|\alpha|} \cdot\left(a_{1}\right)^{-(m+1)} \cdot \delta_{Q^{\prime}}^{|\alpha|-|\beta|}$.

Moreover, since $y$ and $y^{\prime}$ are centers of the distinct $\mathrm{CZ}$ cubes $Q, Q^{\prime}$, we have $\delta_{Q^{\prime}} \leq C^{\prime}\left|y-y^{\prime}\right|$, hence, with $|\beta| \leq|\alpha|$, (107) implies

$$
\left|\partial^{\alpha} \tilde{P}\left(y^{\prime}\right)\right| \cdot\left|\partial^{\beta} P_{\alpha}^{y^{\prime}}\left(y^{\prime}\right)\right| \leq C^{\prime} \cdot\left(a_{1}\right)^{-(m+1)} \cdot \omega\left(\left|y-y^{\prime}\right|\right) \cdot\left|y-y^{\prime}\right|^{m-|\beta|} .
$$

Putting (106) and (108) into (105), and recalling (SU5), we have

$$
\left|\partial^{\beta}\left(P^{\prime}-\tilde{P}\right)\left(y^{\prime}\right)\right| \leq C^{\prime} \cdot\left(a_{1}\right)^{-(m+1)} \cdot \omega\left(\left|y-y^{\prime}\right|\right) \cdot\left|y-y^{\prime}\right|^{m-|\beta|},
$$

for $|\beta| \leq m$.

From (85), (109), and (SU5), we conclude that

$$
\left|\partial^{\beta}\left(P^{\prime}-P\right)\left(y^{\prime}\right)\right| \leq C^{\prime \prime} \cdot\left(a_{1}\right)^{-(m+1)} \cdot \omega\left(\left|y-y^{\prime}\right|\right) \cdot\left|y-y^{\prime}\right|^{m-|\beta|}
$$

for $|\beta| \leq m$. Our results (104) and (110) are the conclusions of Lemma 14.4. The proof of the lemma is complete.

Lemma 14.5 Let $Q, Q^{\prime}$ be distinct $C Z$ cubes, with centers $y, y^{\prime}$, respectively. Let

$$
P \in \mathcal{K}_{f}^{\#}\left(y, k_{A}^{\#}, C\right) \quad \text { and } \quad P^{\prime} \in \mathcal{K}_{f}^{\#}\left(y^{\prime}, k_{A}^{\#}, C\right)
$$

be given, with

$$
k^{\#} \geq(D+1) \cdot k_{A}^{\#}, \quad \text { and } \quad k_{A}^{\#} \geq(D+1)^{2} \cdot k_{\text {old }}^{\#} .
$$

Then we have

$$
\left|\partial^{\beta}\left(P^{\prime}-P\right)\left(y^{\prime}\right)\right| \leq C^{\prime}\left(a_{1}\right)^{-(m+1)} a_{2}^{-1} \omega\left(\left|y-y^{\prime}\right|\right)\left|y-y^{\prime}\right|^{m-|\beta|} \text { for }|\beta| \leq m .
$$


Proof: Let $k_{B}^{\#}=(D+1) \cdot k_{\text {old }}^{\#}$. Then, by Lemma 14.4 , there exists

$$
\tilde{P} \in \mathcal{K}_{f}^{\#}\left(y^{\prime}, k_{B}^{\#}, C^{\prime}\right)
$$

with

$$
\left|\partial^{\beta}(\tilde{P}-P)\left(y^{\prime}\right)\right| \leq C^{\prime} \cdot\left(a_{1}\right)^{-(m+1)} \cdot \omega\left(\left|y-y^{\prime}\right|\right) \cdot\left|y-y^{\prime}\right|^{m-|\beta|} \text { for }|\beta| \leq m .
$$

By $(111)$ and $(114)$, both $P^{\prime}$ and $\tilde{P}$ belong to $\mathcal{K}_{f}^{\#}\left(y^{\prime}, k_{B}^{\#}, C^{\prime \prime}\right)$, with $y^{\prime}$ the center of the $\mathrm{CZ}$ cube $Q^{\prime}$.

Hence, Lemma 14.2 gives

$$
\left|\partial^{\beta}\left(P^{\prime}-\tilde{P}\right)\left(y^{\prime}\right)\right| \leq\left(a_{1}\right)^{-(m+1)} \cdot a_{2}^{-1} \cdot \omega\left(\delta_{Q^{\prime}}\right) \cdot \delta_{Q^{\prime}}^{m-|\beta|} \quad \text { for }|\beta| \leq m .
$$

Since $y$ and $y^{\prime}$ are the centers of distinct CZ cubes $Q, Q^{\prime}$, we have $c \delta_{Q^{\prime}} \leq$ $\left|y-y^{\prime}\right|$, hence also $c \omega\left(\delta_{Q^{\prime}}\right) \leq \omega\left(c \delta_{Q^{\prime}}\right) \leq \omega\left(\left|y-y^{\prime}\right|\right)$ since $\omega$ is a regular modulus of continuity. (Here, we may suppose $c \leq 1$.)

Putting these remarks into (116), we find that

$$
\left|\partial^{\beta}\left(P^{\prime}-\tilde{P}\right)\left(y^{\prime}\right)\right| \leq C^{\prime} \cdot\left(a_{1}\right)^{-(m+1)} \cdot a_{2}^{-1} \cdot \omega\left(\left|y-y^{\prime}\right|\right) \cdot\left|y-y^{\prime}\right|^{m-|\beta|}
$$

for $|\beta| \leq m$.

Adding (115) and (117), and recalling (SU7), we obtain the conclusion (113) of Lemma 14.5. The proof of the lemma is complete.

\section{Patching Local Solutions}

Let $Q_{1}, \ldots, Q_{\mu_{\max }}$ be the $\mathrm{CZ}$ cubes. For $1 \leq \mu \leq \mu_{\max }$, we define $y_{\mu}=$ center $\left(Q_{\mu}\right), \delta_{\mu}=\delta_{Q_{\mu}}=$ diameter $\left(Q_{\mu}\right)$, and

(1) $\tilde{Q}_{\mu}=\left\{y \in \mathbb{R}^{n}: \operatorname{dist}\left(y, Q_{\mu}\right) \leq c_{1} \delta_{\mu}\right\} \subset Q_{\mu}^{*}$, with $c_{1}>0$ a small enough constant depending only on the dimension $n$. Note that $\tilde{Q}_{\mu}$ is not a cube. From the proof of Lemma 11.3, we have the following geometric fact.

(2) If $x \in Q_{\nu}$ and $B\left(x, c_{1} \delta_{\nu}\right)$ meets $\tilde{Q}_{\mu}$, then $Q_{\mu}$ and $Q_{\nu}$ abut or coincide, and, moreover $B\left(x, c_{1} \delta_{\nu}\right) \subset Q_{\mu}^{*}$.

We fix the constant $c_{1}$ as in (1), (2) throughout this section.

We suppose that for each $\mu\left(1 \leq \mu \leq \mu_{\max }\right)$, we are given functions $\theta_{\mu} \in C^{m+1}\left(Q^{\circ}\right)$ and $F_{\mu} \in C^{m}\left(\mathbb{R}^{n}\right)$, and a polynomial $P_{\mu} \in \mathcal{P}$. For a constant $A>0$, not assumed to be a controlled constant, we make the following assumptions. 
(PLS1) $\sum_{1 \leq \mu \leq \mu_{\max }} \theta_{\mu}=1$ on $Q^{\circ}$.

(PLS2) If $x \in Q^{\circ} \backslash \tilde{Q}_{\mu}$, then $\theta_{\mu}=0$ on a neighborhood of $x$ in $Q^{\circ}$.

(PLS3) $\left|\partial^{\beta} \theta_{\mu}(x)\right| \leq A \delta_{\mu}^{-|\beta|}$ for $|\beta| \leq m+1$ and $x \in Q^{\circ}$.

(PLS4) $\left|\partial^{\beta} P_{\mu}\left(y_{\mu}\right)\right| \leq A$ for $|\beta| \leq m$.

(PLS5) $\left|\partial^{\beta}\left(P_{\mu}-P_{\nu}\right)\left(y_{\mu}\right)\right| \leq A \cdot \omega\left(\delta_{\mu}\right) \cdot \delta_{\mu}^{m-|\beta|}$ for $|\beta| \leq m$, if $Q_{\mu}$ and $Q_{\nu}$ abut.

(PLS6) $\left|\partial^{\beta}\left(P_{\mu}-P_{\nu}\right)\left(y_{\mu}\right)\right| \leq A \omega\left(\left|y_{\mu}-y_{\nu}\right|\right) \cdot\left|y_{\mu}-y_{\nu}\right|^{m-|\beta|}$ for $|\beta| \leq m, \mu \neq \nu$.

(PLS7) $\left|\partial^{\beta} F_{\mu}(x)\right| \leq A \omega\left(\delta_{\mu}\right) \cdot \delta_{\mu}^{m-|\beta|}$ for $|\beta| \leq m, x \in Q_{\mu}^{*}$.

(PLS8) $\left|\partial^{\beta} F_{\mu}(\hat{x})-\partial^{\beta} F_{\mu}(\hat{y})\right| \leq A \omega(|\hat{x}-\hat{y}|)$ for $|\beta|=m, \hat{x}, \hat{y} \in Q_{\mu}^{*}$.

Throughout this section, we assume (PLS1, . ,8). In this section only, we write $A^{\prime}, A^{\prime \prime}$, etc., to denote constants determined by $A, m, n$ in $(\mathrm{PLS} 1, \ldots, 8)$. In this section only, we write $c, C, C^{\prime}$, etc. for constants depending only on $m$ and $n$. We reserve the name $c_{1}$ for the constant in (1) and (2).

We define a function $\tilde{F}$ on $Q^{\circ}$, by setting

$$
\tilde{F}=\sum_{1 \leq \mu \leq \mu_{\max }} \theta_{\mu} \cdot\left[P_{\mu}+F_{\mu}\right] \quad \text { on } Q^{\circ} \text {. }
$$

The goal of this section is to control the derivatives of $\tilde{F}$. We begin with a few remarks on the polynomials $P_{\mu}$ and the modulus of continuity $\omega$. First of all, we have

(4) $\left|\partial^{\beta}\left(P_{\mu}-P_{\nu}\right)(x)\right| \leq A^{\prime} \omega\left(\delta_{\mu}\right) \cdot \delta_{\mu}^{m-|\beta|}$ for $|\beta| \leq m, x \in Q_{\mu}^{*}$, if $Q_{\mu}$ and $Q_{\nu}$ coincide or abut.

In fact, when $Q_{\mu}$ and $Q_{\nu}$ abut, then (4) follows from (PLS5) and Taylor's theorem for polynomials, since $\left|x-y_{\mu}\right| \leq C \delta_{\mu}$ for $x \in Q_{\mu}^{*}$. When $Q_{\mu}$ and $Q_{\nu}$ coincide, then $P_{\mu}=P_{\nu}$ and (4) is obvious.

Also,

(5) $\left|\partial^{\beta}\left(P_{\mu}-P_{\nu}\right)(\hat{x})-\partial^{\beta}\left(P_{\mu}-P_{\nu}\right)(\hat{y})\right| \leq A^{\prime} \omega\left(\delta_{\mu}\right) \cdot \delta_{\mu}^{m-|\beta|-1} \cdot|\hat{x}-\hat{y}|$ for $\hat{x}, \hat{y} \in Q_{\mu}^{*},|\beta| \leq m$, if $Q_{\mu}$ and $Q_{\nu}$ abut or coincide.

In fact, when $Q_{\mu}$ and $Q_{\nu}$ abut and $|\beta|<m$, then (5) follows from (PLS5). When $|\beta|=m$ or $Q_{\mu}$ and $Q_{\nu}$ coincide, then the left-hand side of (5) equals zero, so $(5)$ is obvious. (Recall that $P_{\mu}-P_{\nu}$ is a polynomial of degree $\leq m$.) 
Similarly,

(6) $\left|\partial^{\beta} F_{\mu}(\hat{x})-\partial^{\beta} F_{\mu}(\hat{y})\right| \leq A^{\prime} \omega\left(\delta_{\mu}\right) \cdot \delta_{\mu}^{m-|\beta|-1} \cdot|\hat{x}-\hat{y}|$ for $\hat{x}, \hat{y} \in Q_{\mu}^{*}$, $|\beta| \leq m-1$, as follows at once from (PLS7).

We recall that any regular modulus of continuity $\omega$ has the following property.

$$
\text { If } 0 \leq t \leq \delta \leq 1 \text {, with } \delta>0 \text { then } \frac{\omega(\delta)}{\delta} \cdot t \leq \omega(t)
$$

Now we start studying the derivatives of $\tilde{F}$. From the definition (3) of $\tilde{F}$, and from our assumptions on $\theta_{\mu}, F_{\mu}, P_{\mu}$, we see that $\tilde{F}$ belongs to $C^{m}\left(Q^{\circ}\right)$, and we have

$$
\partial^{\beta} \tilde{F}=\sum_{\beta^{\prime}+\beta^{\prime \prime}=\beta} c\left(\beta^{\prime}, \beta^{\prime \prime}\right) \sum_{\mu}\left(\partial^{\beta^{\prime}} \theta_{\mu}\right) \cdot\left(\partial^{\beta^{\prime \prime}}\left[P_{\mu}+F_{\mu}\right]\right) \quad \text { for }|\beta| \leq m,
$$

with $c(0, \beta)=1$. We have also

$$
\sum_{\mu}\left(\partial^{\beta^{\prime}} \theta_{\mu}\right)=\delta_{\beta^{\prime} 0} \quad \text { on } Q^{\circ}
$$

by (PLS1). Hence, (8) implies

$$
\begin{aligned}
\partial^{\beta} \tilde{F}=\partial^{\beta} P_{\nu} & +\sum_{\beta^{\prime}+\beta^{\prime \prime}=\beta} c\left(\beta^{\prime}, \beta^{\prime \prime}\right) \sum_{\mu}\left(\partial^{\beta^{\prime}} \theta_{\mu}\right) \cdot\left(\partial^{\beta^{\prime \prime}}\left[P_{\mu}-P_{\nu}\right]\right) \\
& +\sum_{\beta^{\prime}+\beta^{\prime \prime}=\beta} c\left(\beta^{\prime}, \beta^{\prime \prime}\right) \sum_{\mu}\left(\partial^{\beta^{\prime}} \theta_{\mu}\right)\left(\partial^{\beta^{\prime \prime}} F_{\mu}\right)
\end{aligned}
$$

for $|\beta| \leq m$, and for any $\nu\left(1 \leq \nu \leq \mu_{\max }\right)$. Our estimates below for the derivatives of $\tilde{F}$ are all based on formula (9).

Lemma 15.1 We have

$$
\left|\partial^{\beta} \tilde{F}(x)-\partial^{\beta} P_{\nu}(x)\right| \leq A^{\prime} \omega\left(\delta_{\nu}\right) \cdot \delta_{\nu}^{m-|\beta|} \quad \text { for }|\beta| \leq m, x \in Q_{\nu} .
$$

Proof: Fix $x \in Q_{\nu}$, and suppose $\partial^{\beta^{\prime}} \theta_{\mu}(x) \neq 0$. Then (PLS2) gives

$$
x \in \tilde{Q}_{\mu},
$$

hence (2) shows that

$$
Q_{\mu} \text { and } Q_{\nu} \text { abut or coincide. }
$$


Consequently, Lemma 11.2 implies

$$
\frac{1}{2} \delta_{\nu} \leq \delta_{\mu} \leq 2 \delta_{\nu}
$$

and

(14) There are at most $C$ distinct $\mu$ for which $\partial^{\beta^{\prime}} \theta_{\mu}(x) \neq 0$, for fixed $x$.

Since $\omega$ is a regular modulus of continuity, (13) implies

$$
\frac{1}{2} \omega\left(\delta_{\nu}\right) \leq \omega\left(\delta_{\mu}\right) \leq 2 \omega\left(\delta_{\nu}\right)
$$

With $\mu$ as in $(11), \ldots,(14)$, we estimate the summands in (9). We have

$$
\begin{aligned}
\left|\partial^{\beta^{\prime}} \theta_{\mu}(x)\right| \cdot\left|\partial^{\beta^{\prime \prime}}\left[P_{\mu}-P_{\nu}\right](x)\right| \leq & \left(A \cdot \delta_{\mu}^{-\left|\beta^{\prime}\right|}\right) \cdot\left(A^{\prime} \omega\left(\delta_{\nu}\right) \cdot \delta_{\nu}^{m-\left|\beta^{\prime \prime}\right|}\right) \\
& (\text { by }(\operatorname{PLS} 3),(4),(12)) \\
\leq & A^{\prime \prime} \omega\left(\delta_{\nu}\right) \cdot \delta_{\nu}^{m-|\beta|} \quad \text { (by (13)). }
\end{aligned}
$$

Similarly,

$$
\begin{aligned}
&\left|\partial^{\beta^{\prime}} \theta_{\mu}(x)\right| \cdot\left|\partial^{\beta^{\prime \prime}} F_{\mu}(x)\right| \leq\left(A \delta_{\mu}^{-\left|\beta^{\prime}\right|}\right) \cdot\left(A \omega\left(\delta_{\mu}\right) \cdot \delta_{\mu}^{m-\left|\beta^{\prime \prime}\right|}\right) \\
&(\text { by }(\text { PSL3), (PLS7), (1), (11)) } \\
& \leq A^{\prime} \omega\left(\delta_{\nu}\right) \cdot \delta_{\nu}^{m-|\beta|} \quad(\text { by (13) and (14a)). }
\end{aligned}
$$

Putting (14), ., (16) into (9), we obtain the conclusion (10) of Lemma 15.1. The proof of the Lemma is complete.

Lemma 15.2 Suppose $x \in Q_{\nu}, x^{\prime} \in Q_{\nu^{\prime}},\left|x-x^{\prime}\right| \geq c_{1} \delta_{\nu},\left|x-x^{\prime}\right| \geq c_{1} \delta_{\nu^{\prime}}$. Then we have

$$
\left|\partial^{\beta} \tilde{F}(x)-\partial^{\beta} \tilde{F}\left(x^{\prime}\right)\right| \leq A^{\prime} \omega\left(\left|x-x^{\prime}\right|\right) \text { for }|\beta|=m .
$$

Proof: First of all, note that $x \in Q_{\nu} \subseteq Q^{\circ}$ and $x^{\prime} \in Q_{\nu^{\prime}} \subseteq Q^{\circ}$, hence

$$
\left|x-x^{\prime}\right| \leq \delta_{Q^{\circ}} \leq a_{1} \quad(\operatorname{see}(11.3)) .
$$

In particular, $\left|x-x^{\prime}\right| \leq 1$ by (SU4), so $\omega\left(\left|x-x^{\prime}\right|\right)$ is well-defined.

Next, note that $\partial^{\beta} P_{\nu}$ and $\partial^{\beta} P_{\nu^{\prime}}$ are constant functions on $\mathbb{R}^{n}$, when $|\beta|=m$, since $P_{\nu}, P_{\nu^{\prime}} \in \mathcal{P}$. Consequently, (PLS6) and Lemma 15.1 yield

$$
\begin{aligned}
\left|\partial^{\beta} P_{\nu}-\partial^{\beta} P_{\nu^{\prime}}\right| & \leq A \omega\left(\left|y_{\nu}-y_{\nu^{\prime}}\right|\right), \\
\left|\partial^{\beta} \tilde{F}(x)-\partial^{\beta} P_{\nu}\right| & \leq A^{\prime} \omega\left(\delta_{\nu}\right), \quad \text { and } \\
\left|\partial^{\beta} \tilde{F}\left(x^{\prime}\right)-\partial^{\beta} P_{\nu^{\prime}}\right| & \leq A^{\prime} \omega\left(\delta_{\nu^{\prime}}\right)
\end{aligned}
$$

for $|\beta|=m, x \in Q_{\nu}, x^{\prime} \in Q_{\nu^{\prime}}$. 
Hence, for such $\beta, x, x^{\prime}$, we have

$$
\left|\partial^{\beta} \tilde{F}(x)-\partial^{\beta} \tilde{F}\left(x^{\prime}\right)\right| \leq A \omega\left(\left|y_{\nu}-y_{\nu^{\prime}}\right|\right)+A^{\prime} \omega\left(\delta_{\nu}\right)+A^{\prime} \omega\left(\delta_{\nu^{\prime}}\right) .
$$

Since $x, y_{\nu} \in Q_{\nu}$ and $x^{\prime}, y_{\nu^{\prime}} \in Q_{\nu^{\prime}}$, we have $\left|x-y_{\nu}\right| \leq \delta_{\nu},\left|x^{\prime}-y_{\nu^{\prime}}\right| \leq \delta_{\nu^{\prime}}$, and therefore

$$
\left|y_{\nu}-y_{\nu^{\prime}}\right| \leq\left|x-x^{\prime}\right|+\delta_{\nu}+\delta_{\nu^{\prime}}
$$

Suppose $\left|x-x^{\prime}\right| \geq c_{1} \delta_{\nu}$ and $\left|x-x^{\prime}\right| \geq c_{1} \delta_{\nu^{\prime}}$. Then, in view of (20), we have

$$
\delta_{\nu}, \delta_{\nu^{\prime}},\left|y_{\nu}-y_{\nu^{\prime}}\right| \leq C\left|x-x^{\prime}\right| \leq 1
$$

where the last inequality follows from (18) and (SU5). From (21) and the fact that $\omega$ is a regular modulus of continuity, we obtain the estimates

$$
\omega\left(\delta_{\nu}\right), \omega\left(\delta_{\nu^{\prime}}\right), \omega\left(\left|y_{\nu}-y_{\nu^{\prime}}\right|\right) \leq C \omega\left(\left|x-x^{\prime}\right|\right) .
$$

The desired conclusion (17) is immediate from (19) and (22). The proof of Lemma 15.2 is complete.

Lemma 15.3 Suppose $x \in Q_{\nu}, x^{\prime} \in Q_{\nu^{\prime}}$, and $\left|x-x^{\prime}\right| \leq c_{1} \delta_{\nu}$. Then we have

$$
\left|\partial^{\beta} \tilde{F}(x)-\partial^{\beta} \tilde{F}\left(x^{\prime}\right)\right| \leq A^{\prime} \omega\left(\left|x-x^{\prime}\right|\right) \quad \text { for }|\beta|=m .
$$

Proof: Fix $x \in Q_{\nu}, x^{\prime} \in Q_{\nu^{\prime}}, \beta$ with $|\beta|=m$. Two applications of (9) yield (24) $\partial^{\beta}$

$$
\begin{aligned}
& \partial^{\beta} \tilde{F}(x)-\partial^{\beta} \tilde{F}\left(x^{\prime}\right)=\left[\partial^{\beta} P_{\nu}(x)-\partial^{\beta} P_{\nu}\left(x^{\prime}\right)\right] \\
& +\sum_{\beta^{\prime}+\beta^{\prime \prime}=\beta} c\left(\beta^{\prime}, \beta^{\prime \prime}\right) \sum_{\mu}\left(\partial^{\beta^{\prime}} \theta_{\mu}(x)-\partial^{\beta^{\prime}} \theta_{\mu}\left(x^{\prime}\right)\right) \cdot\left(\partial^{\beta^{\prime \prime}}\left[P_{\mu}-P_{\nu}\right](x)\right) \\
& +\sum_{\beta^{\prime}+\beta^{\prime \prime}=\beta} c\left(\beta^{\prime}, \beta^{\prime \prime}\right) \sum_{\mu}\left(\partial^{\beta^{\prime}} \theta_{\mu}\left(x^{\prime}\right)\right) \cdot\left(\partial^{\beta^{\prime \prime}}\left[P_{\mu}-P_{\nu}\right](x)-\partial^{\beta^{\prime \prime}}\left[P_{\mu}-P_{\nu}\right]\left(x^{\prime}\right)\right) \\
& +\sum_{\beta^{\prime}+\beta^{\prime \prime}=\beta} c\left(\beta^{\prime}, \beta^{\prime \prime}\right) \sum_{\mu}\left(\partial^{\beta^{\prime}} \theta_{\mu}(x)-\partial^{\beta^{\prime}} \theta_{\mu}\left(x^{\prime}\right)\right) \cdot\left(\partial^{\beta^{\prime \prime}} F_{\mu}(x)\right) \\
& +\sum_{\beta^{\prime}+\beta^{\prime \prime}=\beta} c\left(\beta^{\prime}, \beta^{\prime \prime}\right) \sum_{\mu}\left(\partial^{\beta^{\prime}} \theta_{\mu}\left(x^{\prime}\right)\right) \cdot\left(\partial^{\beta^{\prime \prime}} F_{\mu}(x)-\partial^{\beta^{\prime \prime}} F_{\mu}\left(x^{\prime}\right)\right)
\end{aligned}
$$

Suppose $\partial^{\beta^{\prime}} \theta_{\mu}(x)$ or $\partial^{\beta^{\prime}} \theta_{\mu}\left(x^{\prime}\right)$ is non-zero. Then, since $x, x^{\prime} \in B\left(x, c_{1} \delta_{\nu}\right)$, we see from (PLS2) that $B\left(x, c_{1} \delta_{\nu}\right) \cap \tilde{Q}_{\mu} \neq \phi$, with $x \in Q_{\nu}$. Hence, by (2),

$Q_{\mu}$ and $Q_{\nu}$ abut or coincide, and $x, x^{\prime} \in Q_{\mu}^{*}$. 
From (25) and Lemma 11.2, we see that

$$
\frac{1}{2} \delta_{\nu} \leq \delta_{\mu} \leq 2 \delta_{\nu}
$$

and

(28) For our fixed $x, x^{\prime}$ there are at most $C$ distinct $\mu$ for which $\partial^{\beta^{\prime}} \theta_{\mu}(x)$ or $\partial^{\beta^{\prime}} \theta_{\mu}\left(x^{\prime}\right) \neq 0$.

With $\mu$ as in $(25), \ldots,(28)$, and with $\left|\beta^{\prime}\right|+\left|\beta^{\prime \prime}\right|=m$, we estimate the terms on the right in (24).

First of all,

$$
\left[\partial^{\beta} P_{\nu}(x)-\partial^{\beta} P_{\nu}\left(x^{\prime}\right)\right]=0, \quad \text { since } P_{\nu} \in \mathcal{P} \text { and }|\beta|=m .
$$

Next, (PLS3), (4), (25), (26), and (7) imply the estimates

$$
\begin{aligned}
\left|\partial^{\beta^{\prime}} \theta_{\mu}(x)-\partial^{\beta^{\prime}} \theta_{\mu}\left(x^{\prime}\right)\right| & \cdot\left|\partial^{\beta^{\prime \prime}}\left[P_{\mu}-P_{\nu}\right](x)\right| \leq \\
& \leq\left(A^{\prime} \delta_{\mu}^{-\left|\beta^{\prime}\right|-1} \cdot\left|x-x^{\prime}\right|\right) \cdot\left(A^{\prime} \omega\left(\delta_{\mu}\right) \cdot \delta_{\mu}^{m-\left|\beta^{\prime \prime}\right|}\right) \\
& =A^{\prime \prime} \frac{\omega\left(\delta_{\mu}\right)}{\delta_{\mu}}\left|x-x^{\prime}\right| \leq A^{\prime \prime} \omega\left(\left|x-x^{\prime}\right|\right) .
\end{aligned}
$$

Similarly, (PLS3), (5), (25), (26), and (7) imply the estimates

$$
\begin{aligned}
\left|\partial^{\beta^{\prime}} \theta_{\mu}\left(x^{\prime}\right)\right| \cdot \mid \partial^{\beta^{\prime \prime}}\left[P_{\mu}-P_{\nu}\right] & (x)-\partial^{\beta^{\prime \prime}}\left[P_{\mu}-P_{\nu}\right]\left(x^{\prime}\right) \mid \leq \\
\leq & \left(A \delta_{\mu}^{-\left|\beta^{\prime}\right|}\right) \cdot\left(A^{\prime} \omega\left(\delta_{\mu}\right) \cdot \delta_{\mu}^{m-\left|\beta^{\prime \prime}\right|-1} \cdot\left|x-x^{\prime}\right|\right) \\
& =A^{\prime \prime} \frac{\omega\left(\delta_{\mu}\right)}{\delta_{\mu}}\left|x-x^{\prime}\right| \leq A^{\prime \prime} \omega\left(\left|x-x^{\prime}\right|\right) .
\end{aligned}
$$

Also, (PLS3), (PLS7), (26), and (7) imply the estimates

$$
\begin{aligned}
\left|\partial^{\beta^{\prime}} \theta_{\mu}(x)-\partial^{\beta^{\prime}} \theta_{\mu}\left(x^{\prime}\right)\right| & \cdot\left|\partial^{\beta^{\prime \prime}} F_{\mu}(x)\right| \leq \\
& \leq\left(A^{\prime} \delta_{\mu}^{-\left|\beta^{\prime}\right|-1} \cdot\left|x-x^{\prime}\right|\right) \cdot\left(A \omega\left(\delta_{\mu}\right) \cdot \delta_{\mu}^{m-\left|\beta^{\prime \prime}\right|}\right) \\
& =A^{\prime \prime} \frac{\omega\left(\delta_{\mu}\right)}{\delta_{\mu}}\left|x-x^{\prime}\right| \leq A^{\prime \prime} \omega\left(\left|x-x^{\prime}\right|\right) .
\end{aligned}
$$

If $\left|\beta^{\prime \prime}\right|<m$, then (PLS3), (6), (26), and (7) imply the estimate

$$
\begin{aligned}
\left|\partial^{\beta^{\prime}} \theta_{\mu}\left(x^{\prime}\right)\right| \cdot \mid \partial^{\beta^{\prime \prime}} & F_{\mu}(x)-\partial^{\beta^{\prime \prime}} F_{\mu}\left(x^{\prime}\right) \mid \leq \\
& \leq\left(A \delta_{\mu}^{-\left|\beta^{\prime}\right|}\right) \cdot\left(A^{\prime} \omega\left(\delta_{\mu}\right) \cdot \delta_{\mu}^{m-\left|\beta^{\prime \prime}\right|-1} \cdot\left|x-x^{\prime}\right|\right) \\
& =A^{\prime \prime} \frac{\omega\left(\delta_{\mu}\right)}{\delta_{\mu}} \cdot\left|x-x^{\prime}\right| \leq A^{\prime \prime} \omega\left(\left|x-x^{\prime}\right|\right) .
\end{aligned}
$$


If instead $\left|\beta^{\prime \prime}\right|=m$, then $\beta^{\prime}=0$, and (PLS3), (PLS8), (26) yield

$$
\left|\partial^{\beta^{\prime}} \theta_{\mu}\left(x^{\prime}\right)\right| \cdot\left|\partial^{\beta^{\prime \prime}} F_{\mu}(x)-\partial^{\beta^{\prime \prime}} F_{\mu}\left(x^{\prime}\right)\right| \leq(A) \cdot\left(A \omega\left(\left|x-x^{\prime}\right|\right)\right) .
$$

Hence, in either case, we have

$$
\left|\partial^{\beta^{\prime}} \theta_{\mu}\left(x^{\prime}\right)\right| \cdot\left|\partial^{\beta^{\prime \prime}} F_{\mu}(x)-\partial^{\beta^{\prime \prime}} F_{\mu}\left(x^{\prime}\right)\right| \leq A^{\prime \prime} \omega\left(\left|x-x^{\prime}\right|\right) .
$$

Putting (29), ., (33) into (24), and recalling (28), we obtain the desired conclusion (23). The proof of Lemma 15.3 is complete.

Similarly, we have

Lemma 15.4 Suppose $x \in Q_{\nu}, x^{\prime} \in Q_{\nu^{\prime}}$, and $\left|x-x^{\prime}\right| \leq c_{1} \delta_{\nu^{\prime}}$. Then we have $\left|\partial^{\beta} \tilde{F}(x)-\partial^{\beta} \tilde{F}\left(x^{\prime}\right)\right| \leq A^{\prime} \omega\left(\left|x-x^{\prime}\right|\right)$ for $|\beta|=m$.

Proof: This is just Lemma 15.3, with the roles of $x, \nu$ interchanged with those of $x^{\prime}, \nu^{\prime}$.

The main result of this section is as follows.

Lemma 15.5 Let $Q_{\mu}\left(1 \leq \mu \leq \mu_{\max }\right)$ be the $C Z$ cubes, with centers $y_{\mu}$ and diameters $\delta_{\mu}$, and let $\tilde{Q}_{\mu}=\left\{y \in \mathbb{R}^{n}\right.$ : $\left.\operatorname{dist}\left(y, Q_{\mu}\right) \leq c_{1} \delta_{\mu}\right\}$, with $c_{1}$ as in Lemma 11.3. Suppose we are given functions $\theta_{\mu} \in C^{m+1}\left(Q^{\circ}\right), F_{\mu} \in C^{m}\left(\mathbb{R}^{n}\right)$, and polynomials $P_{\mu} \in \mathcal{P}\left(1 \leq \mu \leq \mu_{\max }\right)$. Assume that $(P L S 1, \ldots, 8)$ are satisfied, for a given constant $A$. Define

$$
\tilde{F}=\sum_{1 \leq \mu \leq \mu_{\max }} \theta_{\mu} \cdot\left[P_{\mu}+F_{\mu}\right] \quad \text { on } Q^{\circ} .
$$

Then we have

$$
\left|\partial^{\beta} \tilde{F}(x)\right| \leq A^{\prime} \quad \text { for }|\beta| \leq m, x \in Q^{\circ}
$$

and

$$
\left|\partial^{\beta} \tilde{F}(x)-\partial^{\beta} \tilde{F}\left(x^{\prime}\right)\right| \leq A^{\prime} \cdot \omega\left(\left|x-x^{\prime}\right|\right) \quad \text { for }|\beta|=m, x, x^{\prime} \in Q^{\circ} ;
$$

with $A^{\prime}$ depending only on $A, m, n$.

Proof: Suppose $x \in Q_{\nu}$. Then $\left|\partial^{\beta} P_{\nu}(x)\right| \leq A^{\prime}$ for $|\beta| \leq m$, by (PLS4) and Taylor's theorem for polynomials. Hence, (34) follows from Lemma 15.1.

Next, suppose $x \in Q_{\nu}, x^{\prime} \in Q_{\nu^{\prime}}$. If $\left|x-x^{\prime}\right| \leq c_{1} \delta_{\nu}$ or $\left|x-x^{\prime}\right| \leq c_{1} \delta_{\nu^{\prime}}$, then (35) follows from Lemma 15.3 or Lemma 15.4. If instead $\left|x-x^{\prime}\right| \geq c_{1} \delta_{\nu}$ and $\left|x-x^{\prime}\right| \geq c_{1} \delta_{\nu^{\prime}}$, then (35) follows from Lemma 15.2.

The proof of Lemma 15.5 is complete.

In spirit, the results of this section go back to Whitney [19] and Glaeser [12]. 


\section{Proof of Lemmas 5.2 and 9.1}

In this section, we give the proof of Lemma 9.1. This will also complete the proof of Lemma 5.2, thanks to Lemma 9.2. We are in the setting of Section 9, and we assume (SU0),.., (SU8). As in the previous section, we let $Q_{1}, \ldots, Q_{\mu_{\max }}$ be the CZ cubes, and we set $\delta_{\mu}=\delta_{Q_{\mu}}=\operatorname{diameter}\left(Q_{\mu}\right)$, $y_{\mu}=$ center $\left(Q_{\mu}\right)$.

Recall that

$$
\delta_{\nu} \leq a_{1} \leq 1 \text { for each } \nu
$$

thanks to (11.3).

We take

$$
k^{\#}=(D+1)^{3} \cdot k_{\text {old }}^{\#} .
$$

Lemma 10.5 shows that $\mathcal{K}_{f}^{\#}\left(y_{\nu},(D+1)^{2} \cdot k_{\text {old }}^{\#}, C\right)$ is non-empty for each $\nu$, where $C$ is a large enough controlled constant. For each $\nu$, fix

$$
P_{\nu} \in \mathcal{K}_{f}^{\#}\left(y_{\nu},(D+1)^{2} \cdot k_{\text {old }}^{\#}, C\right) .
$$

Applying Lemmas 14.3 and 14.5, we see that

$$
\left|\partial^{\beta}\left(P_{\mu}-P_{\nu}\right)\left(y_{\mu}\right)\right| \leq C^{\prime} \cdot\left(a_{1}\right)^{-(m+1)} \cdot a_{2}^{-1} \omega\left(\delta_{\nu}\right) \cdot \delta_{\nu}^{m-|\beta|}
$$

for $|\beta| \leq m$, if $Q_{\mu}, Q_{\nu}$ abut; and

(5) $\left|\partial^{\beta}\left(P_{\mu}-P_{\nu}\right)\left(y_{\mu}\right)\right| \leq C^{\prime} \cdot\left(a_{1}\right)^{-(m+1)} \cdot\left(a_{2}\right)^{-1} \cdot \omega\left(\left|y_{\mu}-y_{\nu}\right|\right) \cdot\left|y_{\mu}-y_{\nu}\right|^{m-|\beta|}$ for $|\beta| \leq m, \mu \neq \nu$.

Lemma 16.1 Fix $\nu$. For each $S \subset E \cap Q_{\nu}^{*}$ with $\#(S) \leq k_{\text {old }}^{\#}$, there exists $\hat{F}_{\nu}^{S} \in C^{m, \omega}\left(\mathbb{R}^{n}\right)$, with

$$
\begin{array}{r}
\left|\partial^{\beta} \hat{F}_{\nu}^{S}\left(x^{\prime}\right)\right| \leq C^{\prime} \omega\left(\delta_{\nu}\right) \cdot \delta_{\nu}^{m-|\beta|} \quad \text { for }|\beta| \leq m, x^{\prime} \in \mathbb{R}^{n} ; \\
\left|\partial^{\beta} \hat{F}_{\nu}^{S}\left(x^{\prime}\right)-\partial^{\beta} \hat{F}_{\nu}^{S}\left(x^{\prime \prime}\right)\right| \leq C^{\prime} \omega\left(\left|x^{\prime}-x^{\prime \prime}\right|\right)
\end{array}
$$

for $|\beta|=m, x^{\prime}, x^{\prime \prime} \in \mathbb{R}^{n},\left|x^{\prime}-x^{\prime \prime}\right| \leq \delta_{\nu}$; and

$$
J_{x}\left(\hat{F}_{\nu}^{S}\right) \in\left(f(x)-P_{\nu}\right)+C^{\prime} \sigma(x) \text { for all } x \in S .
$$

(In (8), we regard $P_{\nu}=J_{x}\left(P_{\nu}\right)$ as a jet at $x$.)

Proof: Let $C_{1}$ be a large enough controlled constant, to be fixed in a moment, and let $\hat{\theta}$ be a cutoff function on $\mathbb{R}^{n}$, with

$$
\begin{aligned}
& \hat{\theta}=1 \text { on } Q_{\nu}^{*} \\
& \operatorname{supp} \hat{\theta} \subset B\left(y_{\nu},\left(C_{1}-1\right) \cdot \delta_{\nu}\right) \\
& \left|\partial^{\beta} \hat{\theta}\left(x^{\prime}\right)\right| \leq C^{\prime} \delta_{\nu}^{-|\beta|} \text { for }|\beta| \leq m+1, x^{\prime} \in \mathbb{R}^{n} .
\end{aligned}
$$


In view of $(10)$, we see that

(12) If $\left|x^{\prime}-x^{\prime \prime}\right| \leq \delta_{\nu}$ and at least one of $x^{\prime}, x^{\prime \prime}$ fails to belong to $B\left(y_{\nu}, C_{1} \delta_{\nu}\right)$, then $J_{x^{\prime}}(\hat{\theta})=0, J_{x^{\prime \prime}}(\hat{\theta})=0$.

We pick $C_{1}$ large enough that there exists $\hat{\theta}$ satisfying (9), (10), (11), and we pick $\hat{\theta}$ satisfying these conditions.

Now let $S \subset E \cap Q_{\nu}^{*}$, with $\#(S) \leq k_{\text {old }}^{\#}$. By (3), there exists $F^{S} \in$ $C^{m, \omega}\left(\mathbb{R}^{n}\right)$, with

$$
\begin{aligned}
& \left\|F^{S}\right\|_{C^{m, \omega}\left(\mathbb{R}^{n}\right)} \leq C . \\
& J_{x}\left(F^{S}\right) \in f(x)+C \sigma(x) \text { for all } x \in S, \text { and } \\
& J_{y_{\nu}}\left(F^{S}\right)=P_{\nu} .
\end{aligned}
$$

By (13), we have

$$
\left|\partial^{\beta}\left[F^{S}-P_{\nu}\right]\left(x^{\prime}\right)-\partial^{\beta}\left[F^{S}-P_{\nu}\right]\left(x^{\prime \prime}\right)\right| \leq C \omega\left(\left|x^{\prime}-x^{\prime \prime}\right|\right) \leq C \omega\left(\delta_{\nu}\right)
$$

for $|\beta|=m, x^{\prime}, x^{\prime \prime} \in \mathbb{R}^{n},\left|x^{\prime}-x^{\prime \prime}\right| \leq \delta_{\nu}$.

We have also

$$
J_{y_{\nu}}\left(F^{S}-P_{\nu}\right)=0
$$

by $(15)$.

From (16) and (17), we see that

$$
\left|\partial^{\beta}\left(F^{S}-P_{\nu}\right)\left(x^{\prime}\right)\right| \leq C^{\prime} \omega\left(\delta_{\nu}\right) \cdot \delta_{\nu}^{m-|\beta|}
$$

for $|\beta| \leq m, x^{\prime} \in B\left(y_{\nu}, C_{1} \delta_{\nu}\right)$, with $C_{1}$ as in $(9), \ldots,(12)$.

Also, (14) gives

$$
J_{x}\left(F^{S}-P_{\nu}\right) \in\left(f(x)-P_{\nu}\right)+C \sigma(x) \text { for all } x \in S .
$$

We set

$$
\hat{F}_{\nu}^{S}=\hat{\theta} \cdot\left(F^{S}-P_{\nu}\right)
$$

Since

$$
F^{S} \in C^{m, \omega}\left(\mathbb{R}^{n}\right), P_{\nu} \in \mathcal{P}, \hat{\theta} \in C^{m+1}\left(\mathbb{R}^{n}\right) \text {, and supp } \hat{\theta} \subset B\left(y_{\nu},\left(C_{1}-1\right) \delta_{\nu}\right),
$$

we have $\hat{F}_{\nu}^{S} \in C^{m, \omega}\left(\mathbb{R}^{n}\right)$. We estimate the derivatives of $\hat{F}_{\nu}^{S}$.

Immediately from (11), (18), (20), we obtain

$$
\left|\partial^{\beta} \hat{F}_{\nu}^{S}\left(x^{\prime}\right)\right| \leq C^{\prime} \omega\left(\delta_{\nu}\right) \delta_{\nu}^{m-|\beta|} \text { for }|\beta| \leq m, x^{\prime} \in B\left(y_{\nu}, C_{1} \delta_{\nu}\right) .
$$


Since also $\hat{F}_{\nu}^{S}$ is supported in $B\left(y_{\nu}, C_{1} \delta_{\nu}\right)$ (see (10), (20)), we have

$$
\left|\partial^{\beta} \hat{F}_{\nu}^{S}\left(x^{\prime}\right)\right| \leq C^{\prime} \omega\left(\delta_{\nu}\right) \cdot \delta_{\nu}^{m-|\beta|} \quad \text { for }|\beta| \leq m, x^{\prime} \in \mathbb{R}^{n} .
$$

We estimate $\left|\partial^{\beta} \hat{F}_{\nu}^{S}\left(x^{\prime}\right)-\partial^{\beta} \hat{F}_{\nu}^{S}\left(x^{\prime \prime}\right)\right|$ for $|\beta|=m, x^{\prime}, x^{\prime \prime} \in \mathbb{R}^{n},\left|x^{\prime}-x^{\prime \prime}\right| \leq \delta_{\nu}$.

If either of $x^{\prime}, x^{\prime \prime}$ fails to belong to $B\left(y_{\nu}, C_{1} \delta_{\nu}\right)$, then (12) and (20) give $\partial^{\beta} \hat{F}_{\nu}^{S}\left(x^{\prime}\right)=\partial^{\beta} \hat{F}_{\nu}\left(x^{\prime \prime}\right)=0$. Suppose $x^{\prime}, x^{\prime \prime} \in B\left(y_{\nu}, C_{1} \delta_{\nu}\right)$.

Then (11), (16), (18), (20), and the fact that $\omega$ is a regular modulus of continuity show that

$$
\left|\partial^{\beta} \hat{F}_{\nu}^{S}\left(x^{\prime}\right)-\partial^{\beta} \hat{F}_{\nu}^{S}\left(x^{\prime \prime}\right)\right| \leq C^{\prime} \omega\left(\left|x^{\prime}-x^{\prime \prime}\right|\right) .
$$

To see (22), we write

$$
\begin{aligned}
\partial^{\beta} \hat{F}_{\nu}^{S} & =\hat{\theta} \cdot \partial^{\beta}\left[F^{S}-P_{\nu}\right]+\sum_{\substack{\beta^{\prime}+\beta^{\prime \prime}=\beta \\
\beta^{\prime} \neq 0}} c\left(\beta^{\prime}, \beta^{\prime \prime}\right) \cdot \partial^{\beta^{\prime}} \hat{\theta} \cdot \partial^{\beta^{\prime \prime}}\left[F^{S}-P_{\nu}\right] \\
& \equiv \hat{\theta} \cdot \partial^{\beta}\left[F^{S}-P_{\nu}\right]+G .
\end{aligned}
$$

By (11) and (18), we have $|\nabla G| \leq C^{\prime} \omega\left(\delta_{\nu}\right) \cdot \delta_{\nu}^{m-(|\beta|+1)}=C^{\prime} \omega\left(\delta_{\nu}\right) \cdot \delta_{\nu}^{-1}$, so (23) implies

$$
\begin{gathered}
\left|\partial^{\beta} \hat{F}_{\nu}^{S}\left(x^{\prime}\right)-\partial^{\beta} \hat{F}_{\nu}^{S}\left(x^{\prime \prime}\right)\right| \leq\left|\hat{\theta}\left(x^{\prime}\right) \partial^{\beta}\left[F^{S}-P_{\nu}\right]\left(x^{\prime}\right)-\hat{\theta}\left(x^{\prime \prime}\right) \partial^{\beta}\left[F^{S}-P_{\nu}\right]\left(x^{\prime \prime}\right)\right| \\
+C^{\prime} \omega\left(\delta_{\nu}\right) \delta_{\nu}^{-1}\left|x^{\prime}-x^{\prime \prime}\right| .
\end{gathered}
$$

Moreover,

$$
\begin{aligned}
\mid \hat{\theta}\left(x^{\prime}\right) \partial^{\beta}\left[F^{S}-P_{\nu}\right] & \left(x^{\prime}\right)-\hat{\theta}\left(x^{\prime \prime}\right) \partial^{\beta}\left[F^{S}-P_{\nu}\right]\left(x^{\prime \prime}\right) \mid \leq \\
\leq & \left|\hat{\theta}\left(x^{\prime}\right)\right| \cdot\left|\partial^{\beta}\left[F^{S}-P_{\nu}\right]\left(x^{\prime}\right)-\partial^{\beta}\left[F^{S}-P_{\nu}\right]\left(x^{\prime \prime}\right)\right| \\
& +\left|\hat{\theta}\left(x^{\prime}\right)-\hat{\theta}\left(x^{\prime \prime}\right)\right| \cdot\left|\partial^{\beta}\left[F^{S}-P_{\nu}\right]\left(x^{\prime \prime}\right)\right| \\
\leq & C^{\prime} \omega\left(\left|x^{\prime}-x^{\prime \prime}\right|\right)+C^{\prime} \delta_{\nu}^{-1}\left|x^{\prime}-x^{\prime \prime}\right| \cdot \omega\left(\delta_{\nu}\right)
\end{aligned}
$$

thanks to (11), (16), (18). (Recall that we have here $x^{\prime}, x^{\prime \prime} \in B\left(y_{\nu}, C_{1} \delta_{\nu}\right)$ and $|\beta|=m$.)

From (24) and (25), we have

$$
\left|\partial^{\beta} \hat{F}_{\nu}^{S}\left(x^{\prime}\right)-\partial^{\beta} \hat{F}_{\nu}^{S}\left(x^{\prime \prime}\right)\right| \leq C^{\prime} \omega\left(\left|x^{\prime}-x^{\prime \prime}\right|\right)+C^{\prime} \omega\left(\delta_{\nu}\right) \delta_{\nu}^{-1}\left|x^{\prime}-x^{\prime \prime}\right| .
$$

Since $\left|x^{\prime}-x^{\prime \prime}\right| \leq \delta_{\nu}$ and $\omega$ is a regular modulus of continuity, we have

$$
\omega\left(\delta_{\nu}\right) \delta_{\nu}^{-1}\left|x^{\prime}-x^{\prime \prime}\right| \leq \omega\left(\left|x^{\prime}-x^{\prime \prime}\right|\right)
$$

and therefore, (26) implies (22). The proof of (22) is complete. 
Thus, we have shown that

$$
\left|\partial^{\beta} \hat{F}_{\nu}^{S}\left(x^{\prime}\right)-\partial^{\beta} \hat{F}_{\nu}^{S}\left(x^{\prime \prime}\right)\right| \leq C^{\prime} \omega\left(\left|x^{\prime}-x^{\prime \prime}\right|\right)
$$

for $|\beta|=m, x^{\prime}, x^{\prime \prime} \in \mathbb{R}^{n},\left|x^{\prime}-x^{\prime \prime}\right| \leq \delta_{\nu}$.

Next, suppose $x \in S$. Then we have also $x \in Q_{\nu}^{*}$ (we assumed $S \subset$ $E \cap Q_{\nu}^{*}$ ), hence (9) gives $J_{x}(\hat{\theta})=1$, and therefore (19), (20) show that

$$
J_{x}\left(\hat{F}_{\nu}^{S}\right)=J_{x}\left(F^{S}-P_{\nu}\right) \in\left(f(x)-P_{\nu}\right)+C \sigma(x) .
$$

Thus,

$$
J_{x}\left(\hat{F}_{\nu}^{S}\right) \in\left(f(x)-P_{\nu}\right)+C \sigma(x) \text { for all } x \in S .
$$

We have exhibited $\hat{F}_{\nu}^{S} \in C^{m, \omega}\left(\mathbb{R}^{n}\right)$ satisfying (21), (27), (28). These conditions are precisely the conclusions of Lemma 16.1. The proof of the lemma is complete.

Since $Q_{\nu}$ is a $\mathrm{CZ}$ cube, it is almost $\mathrm{OK}$, i.e., either it is $\mathrm{OK}$ or $Q_{\nu}^{* *}$ contains at most one point of $E$.

Lemma 16.2 Fix $\nu$, and assume that $Q_{\nu}$ is $O K$. For each $y \in Q_{\nu}^{* *}$, let $\overline{\mathcal{A}}^{y}<\mathcal{A}$ and $\bar{P}_{\alpha}^{y} \in \mathcal{P}\left(\alpha \in \overline{\mathcal{A}}^{y}\right)$ be as in $(O K 1,2,3)$ for the cube $Q_{\nu}$.

Then the hypotheses of Lemma 8.1 are satisfied for the following data:

- The constant $A=\left(a_{1}\right)^{-(m+2)}$;

- The cube $Q_{\nu}$;

- The regular modulus of continuity $\omega$;

- The finite set $E \cap Q_{\nu}^{*}$;

- The map $x \mapsto f(x)-P_{\nu} \in \mathcal{R}_{x}$ for $x \in E \cap Q_{\nu}^{*}$;

- The subset $\sigma(x) \subset \mathcal{R}_{x}$ for $x \in E \cap Q_{\nu}^{*}$;

- The set $\overline{\mathcal{A}}^{y}<\mathcal{A}$ for $y \in Q_{\nu}^{* *}$;

- The polynomials $\bar{P}_{\alpha}^{y} \in \mathcal{P}\left(\alpha \in \overline{\mathcal{A}}^{y}\right)$ for $y \in Q_{\nu}^{* *}$.

Proof: The hypotheses of Lemma 8.1 are that the Strong Main Lemma holds for all $\overline{\mathcal{A}}<\mathcal{A}$, and that $(\mathrm{G} 0), \ldots,(\mathrm{G} 4)$ hold.

We are already assuming that the Strong Main Lemma holds for all $\overline{\mathcal{A}}<\mathcal{A}$. (See (SU1).) We check that (G0),.., (G4) hold for our data (as in the statement of Lemma 16.2).

In fact, (G0) for our data says that, for each $x \in E \cap Q_{\nu}^{*}$, the set $\sigma(x)$ is Whitney $\omega$-convex, with Whitney constant $\left(a_{1}\right)^{-(m+2)}$. This follows at once from (SU2) and (SU5). 
Next, (G1) for our data says that

$$
\partial^{\beta} \bar{P}_{\alpha}^{y}(y)=\delta_{\beta \alpha} \quad \text { for } \beta, \alpha \in \overline{\mathcal{A}}^{y}, y \in Q_{\nu}^{* *} .
$$

This is precisely condition (OK1) for the cube $Q_{\nu}$.

Similarly, (G2) for our data says that

$$
\left|\partial^{\beta} \bar{P}_{\alpha}^{y}(y)\right| \leq\left(a_{1}\right)^{-(m+2)} \delta_{\nu}^{|\alpha|-|\beta|} \text { for } \beta \in \mathcal{M}, \alpha \in \overline{\mathcal{A}}^{y}, y \in Q_{\nu}^{* *}, \beta \geq \alpha .
$$

This is precisely condition (OK2) for the cube $Q_{\nu}$.

Next, (G3) for our data says the following.

(29) Given $S \subset E \cap Q_{\nu}^{*}$, with $\#(S) \leq k_{\text {old }}^{\#}$, and given $y \in Q^{* *}$ and $\alpha \in \overline{\mathcal{A}}^{y}$, there exists $\varphi_{\alpha}^{S} \in C_{\ell o c}^{m, \omega}\left(\mathbb{R}^{n}\right)$, with

$$
\begin{aligned}
& \text { (a) } \begin{aligned}
\left|\partial^{\beta} \varphi_{\alpha}^{S}\left(x^{\prime}\right)-\partial^{\beta} \varphi_{\alpha}^{S}\left(x^{\prime \prime}\right)\right| \leq & \left(a_{1}\right)^{-(m+2)} \delta_{\nu}^{|\alpha|-m-1}\left|x^{\prime}-x^{\prime \prime}\right|+ \\
& +a_{0}^{\text {old }}\left(\left(a_{1}\right)^{-(m+2)}\right) \cdot \delta_{\nu}^{|\alpha|-m} \frac{\omega\left(\left|x^{\prime}-x^{\prime \prime}\right|\right)}{\omega\left(\delta_{\nu}\right)} \\
& \text { for }|\beta|=m, x^{\prime}, x^{\prime \prime} \in \mathbb{R}^{n},\left|x^{\prime}-x^{\prime \prime}\right| \leq \delta_{\nu} ;
\end{aligned} \\
& \text { (b) } J_{x}\left(\varphi_{\alpha}^{S}\right) \in\left(a_{1}\right)^{-(m+2)} \delta_{\nu}^{|\alpha|-m}\left(\omega\left(\delta_{\nu}\right)\right)^{-1} \sigma(x) \text { for all } x \in S \text {; and } \\
& \text { (c) } J_{y}\left(\varphi_{\alpha}^{S}\right)=\bar{P}_{\alpha}^{y} .
\end{aligned}
$$

This condition follows at once from (OK3) for $Q_{\nu}$, provided we have

$$
\left(a_{1}\right)^{-(m+2)} \cdot a_{2} \leq a_{0}^{\text {old }}\left(\left(a_{1}\right)^{-(m+2)}\right) .
$$

However, (30) holds, thanks to our assumption (SU7).

Thus, (29) holds as well, and therefore our data satisfy (G3).

Finally, (G4) for our data says the following.

(31) Given $S \subset E \cap Q_{\nu}^{*}$ with $\#(S) \leq k_{\text {old }}^{\#}$, there exists $F^{S} \in C^{m, \omega}\left(\mathbb{R}^{n}\right)$, with
(a) $\left\|\partial^{\beta} F^{S}\right\|_{C^{0}\left(\mathbb{R}^{n}\right)} \leq\left(a_{1}\right)^{-(m+2)} \cdot \omega\left(\delta_{\nu}\right) \cdot \delta_{\nu}^{m-|\beta|}$ for $|\beta| \leq m$;
(b) $\left|\partial^{\beta} F^{S}\left(x^{\prime}\right)-\partial^{\beta} F^{S}\left(x^{\prime \prime}\right)\right| \leq\left(a_{1}\right)^{-(m+2)} \cdot \omega\left(\left|x^{\prime}-x^{\prime \prime}\right|\right)$
for $|\beta|=m, x^{\prime}, x^{\prime \prime} \in \mathbb{R}^{n},\left|x^{\prime}-x^{\prime \prime}\right| \leq \delta_{\nu}$; and
(c) $J_{x}\left(F^{S}\right) \in\left(f(x)-P_{\nu}\right)+\left(a_{1}\right)^{-(m+2)} \sigma(x)$ for all $x \in S$.

However, (31) follows at once from Lemma 16.1 and our assumption (SU5).

Thus (G0),..., (G4) hold for our data.

The proof of Lemma 16.2 is complete. 
Lemma 16.3 For each $\nu\left(1 \leq \nu \leq \mu_{\max }\right)$, there exists $F_{\nu} \in C^{m, \omega}\left(\mathbb{R}^{n}\right)$, with

$$
\begin{aligned}
\left\|\partial^{\beta} F_{\nu}\right\|_{C^{0}\left(\mathbb{R}^{n}\right)} & \leq A^{\prime} \omega\left(\delta_{\nu}\right) \cdot \delta_{\nu}^{m-|\beta|} \text { for }|\beta| \leq m ; \\
\left|\partial^{\beta} F_{\nu}\left(x^{\prime}\right)-\partial^{\beta} F_{\nu}\left(x^{\prime \prime}\right)\right| & \leq A^{\prime} \omega\left(\left|x^{\prime}-x^{\prime \prime}\right|\right)
\end{aligned}
$$

for $|\beta|=m, x^{\prime}, x^{\prime \prime} \in \mathbb{R}^{n},\left|x^{\prime}-x^{\prime \prime}\right| \leq \delta_{\nu} ;$ and

$$
J_{x}\left(F_{\nu}\right) \in\left(f(x)-P_{\nu}\right)+A^{\prime} \sigma(x) \text { for all } x \in E \cap Q_{\nu}^{*} .
$$

Here, $A^{\prime}$ depends only on $a_{1}, m, n$, and the constant $C_{0}$ in $(S U 0, \ldots, 8)$.

Proof: Fix $\nu$. Either $Q_{\nu}$ is $\mathrm{OK}$, or $E \cap Q_{\nu}^{*}$ contains at most one point.

If $Q_{\nu}$ is $\mathrm{OK}$, then the conclusion of Lemma 16.3 is immediate from Lemmas 16.2 and 8.1. If instead there is at most one point in $E \cap Q_{\nu}^{*}$, then the conclusion of Lemma 16.3 is immediate from Lemma 16.1, with $S=E \cap Q_{\nu}^{*}$.

Thus, the lemma holds in all cases.

For each $\nu$, we fix $F_{\nu}$ as in Lemma 16.3. For the rest of this section, we write $A, A^{\prime}, A^{\prime \prime}$, etc. to denote constants determined by $a_{1}, a_{2}, m, n, C_{0}$ in $(\mathrm{SU} 0), \ldots,(\mathrm{SU} 8)$.

We prove a slight variant of (33), namely

$$
\left|\partial^{\beta} F_{\nu}\left(x^{\prime}\right)-\partial^{\beta} F_{\nu}\left(x^{\prime \prime}\right)\right| \leq A^{\prime} \omega\left(\left|x^{\prime}-x^{\prime \prime}\right|\right) \quad \text { for }|\beta|=m, x^{\prime}, x^{\prime \prime} \in Q_{\nu}^{*} .
$$

To see this, recall that $Q_{\nu}^{*}$ has diameter $3 \delta_{\nu}$. Hence, if $x^{\prime}, x^{\prime \prime} \in Q_{\nu}^{*}$, then by subdividing the line segment from $x^{\prime}$ to $x^{\prime \prime}$ into 3 equal parts, we obtain points $x_{0}, x_{1}, x_{2}, x_{3}$, with $x_{0}=x^{\prime}, x_{3}=x^{\prime \prime},\left|x_{i}-x_{i+1}\right|=\frac{1}{3}\left|x^{\prime}-x^{\prime \prime}\right| \leq \delta_{\nu}$.

For $|\beta|=m$, we apply (33) to $x_{i}, x_{i+1}$, to obtain

$$
\left|\partial^{\beta} F_{\nu}\left(x_{i}\right)-\partial^{\beta} F_{\nu}\left(x_{i+1}\right)\right| \leq A^{\prime \prime} \omega\left(\left|x_{i}-x_{i+1}\right|\right) \leq A^{\prime \prime} \omega\left(\left|x-x^{\prime}\right|\right)
$$

for $i=0,1,2$. Summing over $i$, we obtain (35).

Next, we introduce a partition of unity on $Q^{0}$. With a small constant $c_{1}>0$ as in Section 15 (on Patching Local Solutions), we introduce a cutoff function $\tilde{\theta}_{\nu}$ on $\mathbb{R}^{n}$ for each $\nu\left(1 \leq \nu \leq \mu_{\max }\right)$, with

(36) $0 \leq \tilde{\theta}_{\nu} \leq 1$ on $\mathbb{R}^{n}$

(37) $\tilde{\theta}_{\nu}=1$ on $Q_{\nu}$

(38) supp $\tilde{\theta}_{\nu} \subset \tilde{Q}_{\nu}=\left\{y \in \mathbb{R}^{n}\right.$ : dist $\left.\left(y, Q_{\nu}\right) \leq c_{1} \delta_{\nu}\right\} \subset Q_{\nu}^{*}$ (note that $\tilde{Q}_{\nu}$ is not a cube),

$\left|\partial^{\beta} \tilde{\theta}_{\nu}\right| \leq C^{\prime} \delta_{\nu}^{-|\beta|}$ for $|\beta| \leq m+1$ 
We then define

$$
\theta_{\nu}=\bar{\theta}_{\nu} / \sum_{\mu} \tilde{\theta}_{\mu} \quad \text { on } Q^{0}
$$

Note that $\theta_{\nu}$ is defined only on $Q^{0}$. In view of $(36), \ldots,(39)$, we have the following properties of the $\theta_{\nu}$.

$$
\sum_{1 \leq \mu \leq \mu_{\max }} \theta_{\mu}=1 \text { on } Q^{0}
$$

(41) If $x \in Q^{0} \backslash \tilde{Q}_{\mu}$, then $\theta_{\mu}=0$ on a neighborhood of $x$ in $Q^{0}$.

$$
\left|\partial^{\beta} \theta_{\mu}(x)\right| \leq C^{\prime} \delta_{\mu}^{-|\beta|} \text { for }|\beta| \leq m+1, x \in Q^{0},
$$

thanks to Lemma 11.2 and the proof of Lemma 11.3.

We note also a simple consequence of (3), namely

$$
\left|\partial^{\beta} P_{\nu}\left(y_{\nu}\right)\right| \leq C \text { for }|\beta| \leq m \text {, all } \nu .
$$

In fact, (43) follows from (3) by taking $S=$ empty set in the definition of $\mathcal{K}_{f}$ (etc.).

Next, we note that the functions $\theta_{\nu}$ on $Q^{0}, F_{\nu}$ on $\mathbb{R}^{n}$, and the polynomials $P_{\nu}$ satisfy conditions (PLS1, . 8) in the section on Patching Local Solutions, with a constant $A$ determined by $m, n, C_{0}, a_{1}, a_{2}$ in $(\mathrm{SU} 0, \ldots, 8)$.

In fact, (PLS1, 2, 3) are immediate from (40), (41), (42). Also, (PLS4, 5, 6) are immediate from $(43),(4),(5)$ and Lemma 11.2. Finally, (PLS7, 8) are immediate from (32) and (35).

Thus, (PLS1,..,8) hold for the $\theta_{\nu}, F_{\nu}, P_{\nu}$, as claimed.

Therefore, Lemma 15.5 applies to our $\theta_{\nu}, F_{\nu}, P_{\nu}$.

We define

$$
\tilde{F}=\sum_{1 \leq \nu \leq \mu_{\max }} \theta_{\nu} \cdot\left[P_{\nu}+F_{\nu}\right] \quad \text { on } Q^{0}
$$

From Lemma 15.5, we have

$$
\begin{aligned}
\left|\partial^{\beta} \tilde{F}(x)\right| & \leq A^{\prime} \quad \text { for }|\beta| \leq m, x \in Q^{0} ; \text { and } \\
\left|\partial^{\beta} \tilde{F}\left(x^{\prime}\right)-\partial^{\beta} \tilde{F}\left(x^{\prime \prime}\right)\right| & \leq A^{\prime} \omega\left(\left|x^{\prime}-x^{\prime \prime}\right|\right) \quad \text { for }|\beta|=m, x^{\prime}, x^{\prime \prime} \in Q^{0} .
\end{aligned}
$$

Note that $\tilde{F}$ is defined only on $Q^{0}$.

Next, suppose $x \in E \cap Q^{0}$. We pick $\nu\left(1 \leq \nu \leq \mu_{\max }\right)$, with $Q_{\nu}$ containing $x$.

Note that, for any $\mu$ with $x \in Q_{\mu}^{*}$, we have

$$
J_{x}\left(P_{\mu}+F_{\mu}\right) \in f(x)+A^{\prime} \sigma(x)
$$

by (34). In particular, (47) holds whenever $x \in \tilde{Q}_{\mu}$ (see (38)). 
For our given $x$, let $\Omega$ be the set of all $\mu\left(1 \leq \mu \leq \mu_{\max }\right)$ with $x \in \tilde{Q}_{\mu}$. Then (41) and (44) give

$$
J_{x}(\tilde{F})=\sum_{\mu \in \Omega} J_{x}\left(\theta_{\mu}\right) \cdot J_{x}\left(P_{\mu}+F_{\mu}\right)
$$

with the multiplication in (48) performed in $\mathcal{R}_{x}$.

We rewrite (48) in the form

$$
J_{x}(\tilde{F})=J_{x}\left(P_{\nu}+F_{\nu}\right)+\sum_{\mu \in \Omega} J_{x}\left(\theta_{\mu}\right) \cdot\left[J_{x}\left(P_{\mu}+F_{\mu}\right)-J_{x}\left(P_{\nu}+F_{\nu}\right)\right] .
$$

(This holds, thanks to (40).)

We study the summands in (49). Recall that if $x \in Q_{\nu}$ and also $x \in \tilde{Q}_{\mu}$, then $Q_{\nu}$ and $Q_{\mu}$ abut or coincide.

Hence, we have

$$
\begin{aligned}
& \frac{1}{2} \delta_{\nu} \leq \delta_{\mu} \leq 2 \delta_{\nu} \text { for } \mu \in \Omega, \text { by Lemma } 11.2 \text {; and } \\
& \left|\partial^{\beta}\left(P_{\mu}-P_{\nu}\right)\left(y_{\mu}\right)\right| \leq A^{\prime} \omega\left(\delta_{\nu}\right) \delta_{\nu}^{m-|\beta|} \text { for }|\beta| \leq m, \mu \in \Omega \text {, by }(4) .
\end{aligned}
$$

(Of course, (51) holds trivially if $Q_{\mu}$ and $Q_{\nu}$ coincide.)

Since $x, y_{\mu} \in \tilde{Q}_{\mu}$ for $\mu \in \Omega$, we have also $\left|x-y_{\mu}\right| \leq C \delta_{\mu} \leq C^{\prime} \delta_{\nu}$ by (50).

Hence, (51) implies that

$$
\left|\partial^{\beta}\left(P_{\mu}-P_{\nu}\right)(x)\right| \leq A^{\prime} \omega\left(\delta_{\nu}\right) \delta_{\nu}^{m-|\beta|} \quad \text { for }|\beta| \leq m, \mu \in \Omega .
$$

Also, we have

$$
\left|\partial^{\beta} F_{\mu}(x)\right| \leq A^{\prime} \omega\left(\delta_{\mu}\right) \cdot \delta_{\mu}^{m-|\beta|} \leq A^{\prime \prime} \omega\left(\delta_{\nu}\right) \cdot \delta_{\nu}^{m-|\beta|} \quad \text { for }|\beta| \leq m, \mu \in \Omega
$$

thanks to (32), (50), and the fact that $\omega$ is a regular modulus of continuity. In particular, since $x \in Q_{\nu} \subset \tilde{Q}_{\nu}$, we have $\nu \in \Omega$, hence (53) implies

$$
\left|\partial^{\beta} F_{\nu}(x)\right| \leq A^{\prime \prime} \omega\left(\delta_{\nu}\right) \delta_{\nu}^{m-|\beta|} \text { for }|\beta| \leq m .
$$

Estimates (52), (53), (54) show that

$$
\left|\partial^{\beta}\left[J_{x}\left(P_{\mu}+F_{\mu}\right)-J_{x}\left(P_{\nu}+F_{\nu}\right)\right](x)\right| \leq A^{\prime} \omega\left(\delta_{\nu}\right) \cdot \delta_{\nu}^{m-|\beta|}
$$

for $|\beta| \leq m, \mu \in \Omega$.

We have also

$$
J_{x}\left(P_{\mu}+F_{\mu}\right), J_{x}\left(P_{\nu}+F_{\nu}\right) \in f(x)+A^{\prime} \sigma(x),
$$

for $\mu \in \Omega$, by (47) and the fact that $\nu \in \Omega$. 
Hence,

$$
\left[J_{x}\left(P_{\mu}+F_{\mu}\right)-J_{x}\left(P_{\nu}-F_{\nu}\right)\right] \in A^{\prime} \sigma(x) \quad \text { for all } \mu \in \Omega .
$$

From (42), (50) we have also

$$
\left|\partial^{\beta}\left[J_{x}\left(\theta_{\mu}\right)\right](x)\right| \leq C^{\prime} \delta_{\nu}^{-|\beta|} \text { for }|\beta| \leq m, \mu \in \Omega .
$$

Our Whitney $\omega$-convexity assumption (SU2), together with (55), (56), (57), now shows that

$$
J_{x}\left(\theta_{\mu}\right) \cdot\left[J_{x}\left(P_{\mu}+F_{\mu}\right)-J_{x}\left(P_{\nu}+F_{\nu}\right)\right] \in A^{\prime \prime} \sigma(x) \text { for all } \mu \in \Omega .
$$

For each $\mu \in \Omega$, we have $x \in \tilde{Q}_{\mu} \subset Q_{\mu}^{*}$, and $\frac{1}{2} \delta_{\nu} \leq \delta_{\mu} \leq 2 \delta_{\nu}$. Hence, there are at most $C$ distinct $\mu$ in the set $\Omega$.

Consequently, we may sum (58) over all $\mu \in \Omega$. We find that

$$
\sum_{\mu \in \Omega} J_{x}\left(\theta_{\mu}\right) \cdot\left[J_{x}\left(P_{\mu}+F_{\mu}\right)-J_{x}\left(P_{\nu}+F_{\nu}\right)\right] \in A^{\prime} \sigma(x) .
$$

From (47), we have also

$$
J_{x}\left(P_{\nu}+F_{\nu}\right) \in f(x)+A^{\prime} \sigma(x),
$$

since $\nu \in \Omega$. Putting (59), (60) into (49), we find that $J_{x}(\tilde{F}) \in f(x)+A^{\prime} \sigma(x)$. Since we took $x$ to be an arbitrary point of $E \cap Q^{0}$, we have proven that

$$
J_{x}(\tilde{F}) \in f(x)+A^{\prime} \sigma(x) \text { for all } x \in E \cap Q^{0} .
$$

Our function $\tilde{F}$ has the good properties (45), (46), (61), but it is defined only on $Q^{0}$. To remedy this, we multiply $\tilde{F}$ by a cutoff function. We recall (see (11.1), (11.3)) that $Q^{0}$ is centered at $y^{0}$ and has diameter $c a_{1}<\delta_{Q^{0}}<a_{1}$.

We introduce a cutoff function $\theta$ on $\mathbb{R}^{n}$, with

$$
\|\theta\|_{C^{m+1}\left(\mathbb{R}^{n}\right)} \leq A^{\prime}, \theta=1 \quad \text { on } B\left(y^{0}, c^{\prime} a_{1}\right) \text {, supp } \theta \subset Q^{0} .
$$

We then define $F=\theta \cdot \tilde{F}$ on $\mathbb{R}^{n}$. From (45), (46), (62), we obtain $F \in C^{m, \omega}\left(\mathbb{R}^{n}\right)$, with

$$
\|F\|_{C^{m, \omega}\left(\mathbb{R}^{n}\right)} \leq A^{\prime}
$$

and from (61), (62), we have

$$
J_{x}(F) \in f(x)+A^{\prime} \sigma(x) \text { for all } x \in E \cap B\left(y^{0}, c^{\prime} a_{1}\right) .
$$

Since the constants $A^{\prime}$ and $c^{\prime} a_{1}$ in (63), (64) are determined by $m, n, C_{0}, a_{1}, a_{2}$ in (SU0, . 8), our results (63), (64) immediately imply the conclusion of Lemma 9.1.

The proof of Lemma 9.1 is complete.

In view of Lemma 9.2, the proof of Lemma 5.2 is also complete. 


\section{Rescaling Lemmas}

Lemma 17.1 Let $\tau: \mathbb{R}^{n} \rightarrow \mathbb{R}^{n}$ be the linear map

$$
\left(\bar{x}_{1}, \ldots, \bar{x}_{n}\right) \mapsto\left(\lambda_{1} \bar{x}_{1}, \ldots, \lambda_{n} \bar{x}_{n}\right),
$$

with

$$
c_{0}<\lambda_{i} \leq 1 \quad(i=1, \ldots, n) .
$$

Let $\omega$ be a regular modulus of continuity. Let $\bar{x} \in \mathbb{R}^{n}$, and let $x=\tau(\bar{x})$.

Suppose $\sigma \subset \mathcal{R}_{x}$ is Whitney $\omega$-convex, with Whitney constant $C_{0}$.

Define $\bar{\sigma} \subset \mathcal{R}_{\bar{x}}$ by $\bar{\sigma}=\{A P \circ \tau: P \in \sigma\}$, where $A$ is a given positive number.

If $A$ exceeds a large enough constant determined by $c_{0}, m, n$, then $\bar{\sigma}$ is Whitney $\omega$-convex with Whitney constant $C_{0}$.

Proof: We know that $\bar{\sigma}$ is closed, convex, and symmetric about the origin, since $\sigma$ has these properties.

Suppose we are given $\bar{P}, \bar{Q}, \bar{\delta}$, with

$$
\begin{aligned}
& \bar{P} \in \bar{\sigma}, \\
& \bar{Q} \in \mathcal{R}_{\bar{x}}, \\
& 0<\bar{\delta} \leq 1, \\
& \left|\partial^{\beta} \bar{P}(\bar{x})\right| \leq \omega(\bar{\delta}) \cdot \bar{\delta}^{m-|\beta|} \text { for }|\beta| \leq m, \\
& \left|\partial^{\beta} \bar{Q}(\bar{x})\right| \leq \bar{\delta}^{-|\beta|} \text { for }|\beta| \leq m .
\end{aligned}
$$

We must show that

(7) $\quad \bar{P} \cdot \bar{Q} \in C_{0} \bar{\sigma}, \quad$ where the multiplication is performed in $\mathcal{R}_{\bar{x}}$.

We set

$$
P=A^{-1} \bar{P} \circ \tau^{-1} \quad \text { and } \quad Q=\bar{Q} \circ \tau^{-1} .
$$

By (2), (8) and the definition of $\bar{\sigma}$, we have

$$
P \in \sigma .
$$

We have also

$$
\begin{aligned}
|Q(x)| \leq 1, \text { and }\left|\partial^{\beta} Q(x)\right| & \leq C \bar{\delta}^{-|\beta|} \quad \text { for } 1 \leq|\beta| \leq m ; \text { and } \\
\left|\partial^{\beta} P(x)\right| & \leq C A^{-1} \omega(\bar{\delta}) \cdot \bar{\delta}^{m-|\beta|} ;
\end{aligned}
$$

with $C$ in (10), (11) determined by $c_{0}, m, n$. In fact, (10) and (11) follow from (1), (5), (6), (8) and the definition of $\tau$. 
In view of (10), we may find a small constant $\bar{c}$, determined by $c_{0}, m, n$, for which we have

$$
\left|\partial^{\beta} Q(x)\right| \leq(\bar{c} \bar{\delta})^{-|\beta|} \quad \text { for }|\beta| \leq m
$$

and

$$
0<\bar{c} \leq 1
$$

Since $\omega$ is a regular modulus of continuity, we have $\omega(\bar{\delta}) \leq(\bar{c})^{-1} \omega(\bar{c} \delta)$. This estimate and (11) together yield

$$
\left|\partial^{\beta} P(x)\right| \leq \omega(\bar{c} \delta) \cdot(\bar{c} \delta)^{m-|\beta|} \text { for }|\beta| \leq m, \text { with } \bar{c} \text { as in (12), (13), }
$$

provided $A$ exceeds a large enough constant determined by $c_{0}, m, n$.

In view of (4), (13), we have also

$$
0<\bar{c} \bar{\delta} \leq 1
$$

From (9), (12), (14), (15) and the Whitney $\omega$-convexity of $\sigma$, we see that $P \cdot Q \in C_{0} \sigma, \quad$ where the multiplication is performed in $\mathcal{R}_{x}$.

Hence, by definition of $\bar{\sigma}$, we have

$$
A[P \cdot Q] \circ \tau \in C_{0} \bar{\sigma} .
$$

On the other hand, from (8) we have $A P \circ \tau=\bar{P}$ and $Q \circ \tau=\bar{Q}$. Hence,

$$
A[P \cdot Q] \circ \tau=\bar{P} \cdot \bar{Q}
$$

(where, on the right, the multiplication is performed in $\mathcal{R}_{\bar{x}}$ ). Thus,

$$
\bar{P} \cdot \bar{Q} \in C_{0} \bar{\sigma},
$$

which is the desired conclusion (7).

The proof of Lemma 17.1 is complete.

The next lemma is copied from $[9,11]$, and its proof appears in $[9]$.

Recall that $\mathcal{M}^{+}$is the set of multi-indices $\beta=\left(\beta_{1}, \ldots, \beta_{n}\right)$ of order $|\beta|=\beta_{1}+\cdots+\beta_{n} \leq m+1$, while $\mathcal{M}$ is the set of multi-indices $\beta$ of order $|\beta| \leq m$.

Lemma 17.2 Let $\mathcal{A} \subset \mathcal{M}$, and let $C_{1}, \bar{a}$ be positive numbers. Suppose we are given real numbers $F_{\alpha, \beta}$, indexed by $\alpha \in \mathcal{A}$ and $\beta \in \mathcal{M}^{+}$.

Assume that the following conditions are satisfied.

$$
\begin{aligned}
& F_{\alpha, \alpha} \neq 0 \text { for all } \alpha \in \mathcal{A} \text {. } \\
& \left|F_{\alpha, \beta}\right| \leq C_{1}\left|F_{\alpha, \alpha}\right| \text { for all } \alpha \in \mathcal{A}, \beta \in \mathcal{M}^{+} \text {with } \beta \geq \alpha . \\
& F_{\alpha, \beta}=0 \text { for all } \alpha, \beta \in \mathcal{A} \text { with } \alpha \neq \beta \text {. }
\end{aligned}
$$


Then there exist positive numbers $\lambda_{1}, \ldots, \lambda_{n}$ and a map $\phi: \mathcal{A} \rightarrow \mathcal{M}$, with the following properties.

(19) $c<\lambda_{i} \leq 1$, where $c$ is a positive constant determined by $C_{1}, \bar{a}, m, n$.

(20) $\phi(\alpha) \leq \alpha$ for all $\alpha \in \mathcal{A}$.

(21) For each $\alpha \in \mathcal{A}$, either $\phi(\alpha)=\alpha$ or $\phi(\alpha) \notin \mathcal{A}$.

(22) Suppose we define $\hat{F}_{\alpha, \beta}$ for $\alpha \in \mathcal{A}, \beta=\left(\beta_{1}, \ldots, \beta_{n}\right) \in \mathcal{M}^{+}$, by

(a) $\hat{F}_{\alpha, \beta}=\lambda_{1}^{\beta_{1}} \cdots \lambda_{n}^{\beta_{n}} F_{\alpha, \beta}$.

Then we have

(b) $\left|\hat{F}_{\alpha, \beta}\right| \leq \bar{a}_{1}\left|\hat{F}_{\alpha, \phi(\alpha)}\right|$ for all $\alpha \in \mathcal{A}, \beta \in \mathcal{M}^{+}$with $\beta \neq \phi(\alpha)$.

\section{Proof of Lemma 5.3}

In this section, we give the proof of Lemma 5.3. We fix $\mathcal{A} \subseteq \mathcal{M}$, and assume that the Weak Main Lemma holds for all $\overline{\mathcal{A}} \leq \mathcal{A}$. We must show that the Strong Main Lemma holds for $\mathcal{A}$. We may assume that the constant $k^{\#}$ in the Weak Main Lemma for $\overline{\mathcal{A}} \leq \mathcal{A}$ is independent of $\overline{\mathcal{A}}$. (In fact, we may just replace $k_{\overline{\mathcal{A}}}^{\#}$, the value of $k^{\#}$ in the Weak Main Lemma for $\overline{\mathcal{A}}$, by $\max _{\overline{\mathcal{A}} \leq \mathcal{A}} k_{\overline{\mathcal{A}}}^{\#}$.)

Fix $k^{\#}$ as in the Weak Lemma for any $\overline{\mathcal{A}} \leq \mathcal{A}$.

Let $C, \bar{a}_{0}$ be positive constants; let $\omega$ be a regular modulus of continuity; let $E \subset \mathbb{R}^{n}$ be a finite set; let $y^{0}$ be a point of $\mathbb{R}^{n}$; and let $P_{\alpha} \in \mathcal{P}$ be a family of polynomials indexed by $\alpha \in \mathcal{A}$.

Also, suppose that for each $x \in E$ we are given an $m$-jet $f(x) \in \mathcal{R}_{x}$ and a subset $\sigma(x) \subset \mathcal{R}_{x}$. Assume that these data satisfy conditions (SL0, ., 5). We must show that there exists $F \in C^{m, \omega}\left(\mathbb{R}^{n}\right)$, satisfying (SL6,7) with a constant $C^{\prime}$ determined by $C, m, n$.

This will tell us that the Strong Main Lemma holds for $\mathcal{A}$.

Without loss of generality, we may suppose that

$$
y^{0}=0 \text {. }
$$

It will be convenient to introduce two positive constants $\bar{a}$ and $A$, which are assumed to satisfy the following conditions.

(2) $\bar{a}$ is less than a small enough positive constant determined by $C, m, n$.

(3) $A$ exceeds a large enough positive constant determined by $\bar{a}, C, m, n$.

(4) $\bar{a}_{0}$ is less than a small enough positive constant determined by $A$, $\bar{a}, C, m, n$.

Assumptions (2), (3), (4) are not hypotheses of the Strong Main Lemma for $\mathcal{A}$. At the end of our proof, we will remove these assumptions. 
We say that a constant is "controlled" if it is determined by $C, m, n$ in $(\mathrm{SL} 0, \ldots, 5)$. We write $c, C, C^{\prime}$, etc., to denote controlled constants. Similarly, we say that a constant is "controlled by $\bar{a}$ " if it is determined by $\bar{a}$, together with $C, m, n$ in (SL0,.., 5$)$. We write $c(\bar{a}), C(\bar{a}), C^{\prime}(\bar{a})$, etc., to denote constants controlled by $\bar{a}$. Finally, we say that a constant is "controlled by $\bar{a}$ and $A$ " if it is determined by $A, \bar{a}$ and by $C, m, n$ in $(\mathrm{SLO}, \ldots, 5)$. We write $c(\bar{a}, A), C(\bar{a}, A), C^{\prime}(\bar{a}, A)$, etc., to denote constants controlled by $\bar{a}$ and $A$.

Our plan is simply to rescale the problem, using the linear map $\tau: \mathbb{R}^{n} \rightarrow \mathbb{R}^{n}$, given by

$$
\tau:\left(\bar{x}_{1}, \ldots, \bar{x}_{n}\right) \mapsto\left(\lambda_{1} \bar{x}_{1}, \ldots, \lambda_{n} \bar{x}_{n}\right),
$$

for $\lambda_{1}, \ldots, \lambda_{n}>0$ to be picked below. We define

$$
\begin{aligned}
& \bar{E}=\tau^{-1}(E), \bar{P}_{\alpha}=P_{\alpha} \circ \tau, \bar{y}^{0}=0, \\
& \bar{f}(\bar{x})=(f(\tau(\bar{x}))) \circ \tau \in \mathcal{R}_{\bar{x}} \text { for } \bar{x} \in \bar{E},
\end{aligned}
$$

and

$$
\bar{\sigma}(\bar{x})=\{A P \circ \tau: P \in \sigma(\tau(\bar{x}))\} \subset \mathcal{R}_{x} \quad \text { for } \bar{x} \in \bar{E} .
$$

(Note that $(7)$ makes sense, since $f(\tau(\bar{x}))$ is an $m$-jet at $\tau(\bar{x})$.)

We keep $\omega$ unchanged.

Thus, $\omega$ is a regular modulus of continuity, $\bar{E}$ is a finite subset of $\mathbb{R}^{n}$, $\overline{y^{0}}$ is point of $\mathbb{R}^{n}, \bar{P}_{\alpha} \in \mathcal{P}$ is a polynomial indexed by $\alpha \in \mathcal{A}$; and for each $\bar{x} \in \bar{E}, \bar{f}(\bar{x}) \in \mathcal{R}_{\bar{x}}$ is an $m$-jet and $\bar{\sigma}(\bar{x}) \subset \mathcal{R}_{\bar{x}}$.

Evidently,

(9) $\partial^{\beta} \bar{P}_{\alpha}\left(\bar{y}^{0}\right)=\lambda_{1}^{\beta_{1}} \cdots \lambda_{n}^{\beta_{n}} \partial^{\beta} P_{\alpha}\left(y^{0}\right) \quad$ for $\alpha \in \mathcal{A}, \beta=\left(\beta_{1}, \ldots, \beta_{n}\right) \in \mathcal{M}$

To pick $\lambda_{1}, \ldots, \lambda_{n}$, we appeal to Lemma 17.2 , with

$$
F_{\alpha, \beta}=\partial^{\beta} P_{\alpha}\left(y^{0}\right) \quad \text { for } \alpha \in \mathcal{A}, \beta \in \mathcal{M},
$$

and

$$
F_{\alpha, \beta}=1 \quad \text { for } \alpha \in \mathcal{A},|\beta|=m+1 .
$$

Note that the hypotheses of Lemma 17.2 are satisfied here, with $\bar{a}$ as in (2), (3), (4); and with $C_{1}$ a controlled constant. In fact, (SL1) shows that $F_{\alpha, \alpha} \neq 0$ for all $\alpha \in \mathcal{A}$. Also, (SL1,2) and (10), (11) show that

$$
\left|F_{\alpha, \beta}\right| \leq C_{1}\left|F_{\alpha, \alpha}\right|
$$

for $\alpha \in \mathcal{A}, \beta \in \mathcal{M}^{+}, \beta>\alpha$.

Finally, $F_{\alpha, \beta}=0$ if $\alpha, \beta \in \mathcal{A}$ and $\alpha \neq \beta$, thanks to (SL1) and (10).

Thus, the hypotheses of Lemma 17.2 are satisfied by $\bar{a}, C_{1}, F_{\alpha, \beta}$ as claimed. 
Applying that lemma, we obtain positive numbers $\lambda_{1}, \ldots, \lambda_{n}$ and a map $\phi: \mathcal{A} \rightarrow \mathcal{M}$, with the following properties.

(12) $c(\bar{a})<\lambda_{i} \leq 1$ for each $i=1, \ldots, n$.

(13) $\phi(\alpha) \leq \alpha$ for each $\alpha \in \mathcal{A}$.

(14) For each $\alpha \in \mathcal{A}$, either $\phi(\alpha)=\alpha$ or $\phi(\alpha) \notin \mathcal{A}$.

(15) For any $\alpha \in \mathcal{A}, \beta \in \mathcal{M}$ with $\beta \neq \phi(\alpha)$, we have $\left|\partial^{\beta} \bar{P}_{\alpha}\left(\bar{y}^{0}\right)\right| \leq \bar{a} \cdot\left|\partial^{\phi(\alpha)} \bar{P}_{\alpha}\left(\bar{y}^{0}\right)\right|$.

(16) For any $\alpha \in \mathcal{A}$, we have $\lambda_{1}^{\beta_{1}} \cdots \lambda_{n}^{\beta_{n}} \leq \bar{a} \cdot\left|\partial^{\phi(\alpha)} \bar{P}_{\alpha}\left(\bar{y}^{0}\right)\right|$ for $\beta_{1}+\cdots+\beta_{n}=m+1$.

Here, (15) and (16) follow from the conclusions of Lemma 17.2, together with (9), (10), (11).

Let $\bar{S} \subset \bar{E}$ be given, with $\#(\bar{S}) \leq k^{\#}$. Set $S=\tau(\bar{S})$, and apply (SL3). Let $\varphi_{\alpha}^{S}(\alpha \in \mathcal{A})$ be as in (SL3), and define

$$
\bar{\varphi}_{\alpha}^{\bar{S}}=\varphi_{\alpha}^{S} \circ \tau, \quad \text { for } \alpha \in \mathcal{A} .
$$

Thus, $\bar{\varphi}_{\alpha}^{S} \in C_{\ell o c}^{m, \omega}\left(\mathbb{R}^{n}\right)$, since $\varphi_{\alpha}^{S} \in C_{\ell o c}^{m, \omega}\left(\mathbb{R}^{n}\right)$.

For $\beta=\left(\beta_{1}, \ldots, \beta_{n}\right)$ with $|\beta|=m$, and for $\bar{x}^{\prime}=\left(\bar{x}_{1}^{\prime}, \ldots, \bar{x}_{n}^{\prime}\right), \bar{x}^{\prime \prime}=$ $\left(\bar{x}_{1}^{\prime \prime}, \ldots, \bar{x}_{n}^{\prime \prime}\right)$ in $\mathbb{R}^{n}$ with $\left|\bar{x}^{\prime}-\bar{x}^{\prime \prime}\right| \leq 1$, we have $\left|\tau\left(\bar{x}^{\prime}\right)-\tau\left(\bar{x}^{\prime \prime}\right)\right| \leq 1$ (see (5) and (12)), hence

$$
\begin{aligned}
& \left|\partial^{\beta} \bar{\varphi}_{\alpha}^{\bar{S}}\left(\bar{x}^{\prime}\right)-\partial^{\beta} \bar{\varphi}_{\alpha}^{\bar{S}}\left(\bar{x}^{\prime \prime}\right)\right|=\lambda_{1}^{\beta_{1}} \cdots \lambda_{n}^{\beta_{n}}\left|\partial^{\beta} \varphi_{\alpha}^{S}\left(\tau\left(\bar{x}^{\prime}\right)\right)-\partial^{\beta} \varphi_{\alpha}^{S}\left(\tau\left(\bar{x}^{\prime \prime}\right)\right)\right| \\
& \quad \leq \lambda_{1}^{\beta_{1}} \cdots \lambda_{n}^{\beta_{n}} \cdot\left[\bar{a}_{0} \omega\left(\left|\tau\left(\bar{x}^{\prime}\right)-\tau\left(\bar{x}^{\prime \prime}\right)\right|\right)+C\left|\tau\left(\bar{x}^{\prime}\right)-\tau\left(\bar{x}^{\prime \prime}\right)\right|\right] \quad \text { by }((\text { SL3 })(\mathrm{a})) \\
& \quad \leq \bar{a}_{0} \omega\left(\left|\bar{x}^{\prime}-\bar{x}^{\prime \prime}\right|\right)+C \lambda_{1}^{\beta_{1}} \cdots \lambda_{n}^{\beta_{n}} \sum_{j=1}^{n} \lambda_{j}\left|\bar{x}_{j}^{\prime}-\bar{x}_{j}^{\prime \prime}\right| \quad(\text { by }(5),(12)) \\
& \quad \leq \bar{a}_{0} \omega\left(\left|\bar{x}^{\prime}-\bar{x}^{\prime \prime}\right|\right)+C \bar{a}\left|\partial^{\phi(\alpha)} \bar{P}_{\alpha}\left(\bar{y}^{0}\right)\right| \cdot \sum_{j=1}^{n}\left|\bar{x}_{j}^{\prime}-\bar{x}_{j}^{\prime \prime}\right| \quad(\text { by }(16)) \\
& \quad \leq \bar{a}_{0} \omega\left(\left|\bar{x}^{\prime}-\bar{x}^{\prime \prime}\right|\right)+C^{\prime} \bar{a}\left|\partial^{\phi(\alpha)} \bar{P}_{\alpha}\left(\bar{y}^{0}\right)\right| \cdot\left|\bar{x}^{\prime}-\bar{x}^{\prime \prime}\right| \\
& \quad \leq \bar{a}_{0} \omega\left(\left|\bar{x}^{\prime}-\bar{x}^{\prime \prime}\right|\right)+C^{\prime} \bar{a}\left|\partial^{\phi(\alpha)} \bar{P}_{\alpha}\left(\bar{y}^{0}\right)\right| \cdot \omega\left(\left|\bar{x}^{\prime}-\bar{x}^{\prime \prime}\right|\right),
\end{aligned}
$$

since $\omega$ is a regular modulus of continuity, and hence $\frac{\omega(t)}{t} \geq \frac{\omega(1)}{1}=1$ for $0<t \leq 1($ also $\omega(0)=0)$. Thus,

(18) for $|\beta|=m, \bar{x}^{\prime}, \bar{x}^{\prime \prime} \in \mathbb{R}^{n},\left|\bar{x}^{\prime}-\bar{x}^{\prime \prime}\right| \leq 1$, we have

$$
\left|\partial^{\beta} \bar{\varphi}_{\alpha}^{\bar{S}}\left(\bar{x}^{\prime}\right)-\partial^{\beta} \bar{\varphi}_{\alpha}^{\bar{S}}\left(\bar{x}^{\prime \prime}\right)\right| \leq\left[\bar{a}_{0}+C^{\prime} \bar{a}\left|\partial^{\phi(\alpha)} \bar{P}_{\alpha}\left(\bar{y}^{0}\right)\right|\right] \cdot \omega\left(\left|\bar{x}^{\prime}-\bar{x}^{\prime \prime}\right|\right) .
$$


Also, from (9), (15), (SL1), (12), we have, for $\alpha=\left(\alpha_{1}, \ldots, \alpha_{n}\right) \in \mathcal{A}$, that

$$
\begin{aligned}
\left|\partial^{\phi(\alpha)} \bar{P}_{\alpha}\left(\bar{y}^{0}\right)\right| & \geq\left|\partial^{\alpha} \bar{P}_{\alpha}\left(\bar{y}^{0}\right)\right|=\lambda_{1}^{\alpha_{1}} \cdots \lambda_{n}^{\alpha_{n}}\left|\partial^{\alpha} P_{\alpha}\left(y^{0}\right)\right| \\
& =\lambda_{1}^{\alpha_{1}} \cdots \lambda_{n}^{\alpha_{n}} \geq c(\bar{a}) .
\end{aligned}
$$

From (18), (19), we have the following.

$$
\left|\partial^{\beta} \bar{\varphi}_{\alpha}^{\bar{S}}\left(\bar{x}^{\prime}\right)-\partial^{\beta} \bar{\varphi}_{\alpha}^{\bar{S}}\left(\bar{x}^{\prime \prime}\right)\right| \leq\left[C(\bar{a}) \cdot \bar{a}_{0}+C^{\prime} \cdot \bar{a}\right] \cdot\left|\partial^{\phi(\alpha)} \bar{P}_{\alpha}\left(\bar{y}^{0}\right)\right| \cdot \omega\left(\left|\bar{x}^{\prime}-\bar{x}^{\prime \prime}\right|\right)
$$

for $\alpha \in \mathcal{A},|\beta|=m, \bar{x}^{\prime}, \bar{x}^{\prime \prime} \in \mathbb{R}^{n},\left|\bar{x}^{\prime}-\bar{x}^{\prime \prime}\right| \leq 1$. have

Also, (SL3)(b), together with (17) and (8), shows that, for $\bar{x} \in \bar{S}$, we

$$
J_{\bar{x}}\left(\bar{\varphi}_{\alpha}^{\bar{S}}\right)=\left[J_{\tau(\bar{x})}\left(\varphi_{\alpha}^{S}\right)\right] \circ \tau \in\{P \circ \tau: P \in C \sigma(\tau(\bar{x}))\}=C A^{-1} \bar{\sigma}(\bar{x}) .
$$

Thus,

$$
J_{\bar{x}}\left(\bar{\varphi}_{\alpha}^{\bar{S}}\right) \in C A^{-1} \bar{\sigma}(\bar{x}) \quad \text { for all } \alpha \in \mathcal{A}, \bar{x} \in \bar{S} .
$$

From (SL3)(c), (17), (6), we have

$$
J_{\bar{y}^{0}}\left(\bar{\varphi}_{\alpha}^{\bar{S}}\right)=\left[J_{y^{0}}\left(\varphi_{\alpha}^{S}\right)\right] \circ \tau=P_{\alpha} \circ \tau=\bar{P}_{\alpha} .
$$

Thus,

$$
J_{\bar{y}^{0}}\left(\bar{\varphi}_{\alpha}^{\bar{S}}\right)=\bar{P}_{\alpha} \quad \text { for all } \alpha \in \mathcal{A}
$$

Since $\bar{\varphi} \bar{S}_{\alpha}^{\bar{S}}$ satisfies (20), (21), (22), we have proven the following.

(23) Given $\bar{S} \subset \bar{E}$ with $\#(\bar{S}) \leq k^{\#}$, and given $\alpha \in \mathcal{A}$, there exists $\bar{\varphi}_{\alpha}^{\bar{S}} \in$ $C_{\ell o c}^{m, \omega}\left(\mathbb{R}^{n}\right)$, with
(a) $\left|\partial^{\beta} \bar{\varphi}_{\alpha}^{\bar{S}}\left(\bar{x}^{\prime}\right)-\partial^{\beta} \bar{\varphi}_{\alpha}^{\bar{S}}\left(\bar{x}^{\prime \prime}\right)\right| \leq\left[C(\bar{a}) \cdot \bar{a}_{0}+C^{\prime} \bar{a}\right] \cdot\left|\partial^{\phi(\alpha)} \bar{P}_{\alpha}\left(y^{0}\right)\right| \cdot \omega\left(\left|\bar{x}^{\prime}-\bar{x}^{\prime \prime}\right|\right)$ for $|\beta|=m, \bar{x}^{\prime}, \bar{x}^{\prime \prime} \in \mathbb{R}^{n},\left|\bar{x}^{\prime}-\bar{x}^{\prime \prime}\right| \leq 1$;
(b) $J_{\bar{x}}\left(\bar{\varphi}_{\alpha}^{\bar{S}}\right) \in C A^{-1} \bar{\sigma}(\bar{x})$ for all $\bar{x} \in \bar{S}$; and
(c) $J_{\bar{y}^{0}}\left(\bar{\varphi}_{\alpha}^{\bar{S}}\right)=\bar{P}_{\alpha}$

Similarly, let $\bar{S} \subset \bar{E}$ be given, with $\#(\bar{S}) \leq k^{\#}$. Again, we set $S=\tau(\bar{S})$, and we apply (SL4). Let $F^{S}$ be as in (SL4), and define

$$
\bar{F}^{\bar{S}}=F^{S} \circ \tau \text {. }
$$

Thus, $\bar{F}^{\bar{S}} \in C^{m, \omega}\left(\mathbb{R}^{n}\right)$, since $F^{S} \in C^{m, \omega}\left(\mathbb{R}^{n}\right)$. 
For $\beta=\left(\beta_{1}, \ldots, \beta_{n}\right)$ with $|\beta| \leq m,($ SL4)(a) and (112) give

$$
\left\|\partial^{\beta} \bar{F}^{\bar{S}}\right\|_{C^{0}\left(\mathbb{R}^{n}\right)}=\lambda_{1}^{\beta_{1}} \cdots \lambda_{n}^{\beta_{n}}\left\|\partial^{\beta} F^{S}\right\|_{C^{0}\left(\mathbb{R}^{n}\right)} \leq C .
$$

Also, for $\beta=\left(\beta_{1}, \ldots, \beta_{n}\right)$ with $|\beta|=m$, and with $\bar{x}^{\prime}, \bar{x}^{\prime \prime} \in \mathbb{R}^{n}$ with $\left|\bar{x}^{\prime}-\bar{x}^{\prime \prime}\right| \leq 1$, we have $\left|\tau\left(\bar{x}^{\prime}\right)-\tau\left(\bar{x}^{\prime \prime}\right)\right| \leq 1$ by (12), hence (SL4)(a) and (12) give

$$
\begin{aligned}
\mid \partial^{\beta} \bar{F}^{\bar{S}}\left(\bar{x}^{\prime}\right) & -\partial^{\beta} \bar{F}^{\bar{S}}\left(\bar{x}^{\prime \prime}\right)\left|=\lambda_{1}^{\beta_{1}} \cdots \lambda_{n}^{\beta_{n}}\right| \partial^{\beta} F^{S}\left(\tau\left(\bar{x}^{\prime}\right)\right)-\partial^{\beta} F^{S}\left(\tau\left(\bar{x}^{\prime \prime}\right)\right) \mid \\
& \leq \lambda_{1}^{\beta_{1}} \cdots \lambda_{n}^{\beta_{n}} \cdot C \omega\left(\left|\tau\left(\bar{x}^{\prime}\right)-\tau\left(\bar{x}^{\prime \prime}\right)\right|\right) \leq C \omega\left(\left|\bar{x}^{\prime}-\bar{x}^{\prime \prime}\right|\right) .
\end{aligned}
$$

From (25), (26), we see that

$$
\left\|\bar{F}^{\bar{S}}\right\|_{C^{m, \omega}\left(\mathbb{R}^{n}\right)} \leq C .
$$

Suppose $\bar{x} \in \bar{S}$. Then (24), (SL4)(b), (7), (8) give

$$
\begin{aligned}
J_{\bar{x}}\left(\bar{F}^{\bar{S}}\right) & =\left[J_{\tau(\bar{x})}\left(F^{S}\right)\right] \circ \tau \in[f(\tau(\bar{x}))+C \sigma(\tau(\bar{x}))] \circ \tau= \\
& =f(\tau(\bar{x})) \circ \tau+\{C P \circ \tau: P \in \sigma(\tau(\bar{x}))\}=\bar{f}(\bar{x})+C A^{-1} \bar{\sigma}(\bar{x}) .
\end{aligned}
$$

Thus,

$$
J_{\bar{x}}\left(\bar{F}^{\bar{S}}\right) \in \bar{f}(\bar{x})+C A^{-1} \bar{\sigma}(\bar{x}) \quad \text { for all } \bar{x} \in \bar{S} .
$$

Since $\bar{F}^{\bar{S}}$ satisfies (27) and (28), we have proven the following.

(29) Given $\bar{S} \subset \bar{E}$ with $\#(\bar{S}) \leq k^{\#}$, there exists $\bar{F}^{\bar{S}} \in C^{m, \omega}\left(\mathbb{R}^{n}\right)$, with

$\left\|\bar{F}^{\bar{S}}\right\|_{C^{m, \omega}\left(\mathbb{R}^{n}\right)} \leq C$, and $J_{\bar{x}}\left(\bar{F}^{\bar{S}}\right) \in \bar{f}(\bar{x})+C A^{-1} \bar{\sigma}(\bar{x})$ for all $\bar{x} \in \bar{S}$.

Now define

$$
\overline{\mathcal{A}}=\phi(\mathcal{A})
$$

and let $\psi: \overline{\mathcal{A}} \rightarrow \mathcal{A}$ satisfy

$$
\phi(\psi(\bar{\alpha}))=\bar{\alpha} \quad \text { for all } \bar{\alpha} \in \overline{\mathcal{A}} .
$$

Note that

$$
\overline{\mathcal{A}} \leq \mathcal{A}
$$

by (13), (14), (30), and Lemma 3.3.

For each $\bar{\alpha} \in \overline{\mathcal{A}}$, define

$$
\tilde{P}_{\bar{\alpha}}=\bar{P}_{\psi(\bar{\alpha})} /\left(\partial^{\bar{\alpha}} \bar{P}_{\psi(\bar{\alpha})}\left(\bar{y}^{0}\right)\right) \in \mathcal{P} .
$$


Note that, with $\alpha=\psi(\bar{\alpha})$, we have

$$
\left|\partial^{\bar{\alpha}} \bar{P}_{\psi(\bar{\alpha})}\left(\bar{y}^{0}\right)\right|=\left|\partial^{\phi(\alpha)} \bar{P}_{\alpha}\left(\bar{y}^{0}\right)\right| \geq c(\bar{a}) \quad \text { for } \bar{\alpha} \in \overline{\mathcal{A}}, \quad \text { by }(19) .
$$

Hence, the denominator in (33) is non-zero.

We derive the basic properties of the $\tilde{P}_{\bar{\alpha}}$ for $\bar{\alpha} \in \overline{\mathcal{A}}$.

From (15), with $\alpha=\psi(\bar{\alpha})$, we have, for $\bar{\alpha} \in \overline{\mathcal{A}}, \beta \in \mathcal{M}, \beta \neq \bar{\alpha}$, that

$$
\left|\partial^{\beta} \tilde{P}_{\bar{\alpha}}\left(\bar{y}^{0}\right)\right|=\left|\partial^{\beta} \bar{P}_{\alpha}\left(\bar{y}^{0}\right)\right| /\left|\partial^{\phi(\alpha)} \bar{P}_{\alpha}\left(y^{0}\right)\right| \leq \bar{a} \quad(\text { since } \bar{\alpha}=\phi(\alpha)) .
$$

Also, for $\bar{\alpha} \in \overline{\mathcal{A}}$, we have

$$
\partial^{\bar{\alpha}} \tilde{P}_{\bar{\alpha}}\left(\bar{y}^{0}\right)=\left(\partial^{\bar{\alpha}} \bar{P}_{\psi(\bar{\alpha})}\left(\bar{y}^{0}\right)\right) /\left(\partial^{\bar{\alpha}} \bar{P}_{\psi(\bar{\alpha})}\left(\bar{y}^{0}\right)\right)=1 .
$$

Hence,

$$
\left|\partial^{\beta} \tilde{P}_{\bar{\alpha}}\left(\bar{y}^{0}\right)-\delta_{\beta \alpha}\right| \leq \bar{a} \quad \text { for all } \bar{\alpha} \in \overline{\mathcal{A}}, \beta \in \mathcal{M}
$$

Also, from (23) (with $\alpha=\psi(\bar{\alpha})$ ), (19) and (33), we obtain the following.

(36) Given $\bar{\alpha} \in \overline{\mathcal{A}}$ and $\bar{S} \subset \bar{E}$ with $\#(\bar{S}) \leq k^{\#}$, there exists $\tilde{\varphi}_{\bar{\alpha}}^{\bar{S}} \in C_{\ell o c}^{m, \omega}\left(\mathbb{R}^{n}\right)$, with

(a) $\left|\partial^{\beta} \tilde{\varphi} \overline{\bar{\alpha}}\left(\bar{x}^{\prime}\right)-\partial^{\beta} \tilde{\varphi}_{\bar{\alpha}}^{\bar{S}}\left(\bar{x}^{\prime \prime}\right)\right| \leq\left[C(\bar{a}) \cdot \overline{a_{0}}+C^{\prime} \bar{a}\right] \cdot \omega\left(\left|\bar{x}^{\prime}-\bar{x}^{\prime \prime}\right|\right)$

for $|\beta|=m, \bar{x}^{\prime}, \bar{x}^{\prime \prime} \in \mathbb{R}^{n},\left|\bar{x}^{\prime}-\bar{x}^{\prime \prime}\right| \leq 1$;

(b) $J_{\bar{x}}\left(\tilde{\varphi}_{\bar{\alpha}}^{\bar{S}}\right) \in C(\bar{a}) \cdot A^{-1} \bar{\sigma}(\bar{x})$ for all $\bar{x} \in S$; and

(c) $J_{\bar{y}^{0}}\left(\tilde{\varphi}_{\bar{\alpha}}^{\bar{S}}\right)=\tilde{P}_{\bar{\alpha}}$.

(In fact, we just set $\tilde{\varphi}_{\bar{\alpha}}^{\bar{S}}=\bar{\varphi}_{\alpha}^{\bar{S}} /\left(\partial^{\bar{\alpha}} \bar{P}_{\psi(\bar{\alpha})}\left(\bar{y}^{0}\right)\right)$ with $\alpha=\psi(\bar{\alpha})$ and $\bar{\varphi} \overline{\bar{\alpha}}$ as in (23).)

From (35) and (2), we see that the matrix $\left(\partial^{\beta} \tilde{P}_{\bar{\alpha}}\left(\bar{y}^{0}\right)\right)_{\beta, \bar{\alpha} \in \overline{\mathcal{A}}}$ has an inverse $\left(M_{\alpha^{\prime}, \bar{\alpha}}\right)_{\alpha^{\prime}, \bar{\alpha} \in \overline{\mathcal{A}}}$, with

$$
\left|M_{\alpha^{\prime} \bar{\alpha}}-\delta_{\alpha^{\prime} \bar{\alpha}}\right| \leq C \bar{a} \quad \text { for } \alpha^{\prime}, \bar{\alpha} \in \overline{\mathcal{A}} .
$$

We now define

$$
\stackrel{\bar{P}}{\bar{\alpha}}_{\bar{\alpha}}=\sum_{\alpha^{\prime} \in \overline{\mathcal{A}}} \tilde{P}_{\alpha^{\prime}} \cdot M_{\alpha^{\prime} \bar{\alpha}} \in \mathcal{P} \quad \text { for all } \bar{\alpha} \in \overline{\mathcal{A}} .
$$

From (37), (38), we have

$$
\partial^{\beta} \stackrel{\overline{\bar{P}}}{\bar{\alpha}}_{\bar{y}}\left(\bar{y}^{0}\right)=\delta_{\beta \bar{\alpha}} \quad \text { for } \beta, \bar{\alpha} \in \overline{\mathcal{A}} .
$$


Also, from (35), (30), and (2), we have

$$
\left|\partial^{\beta} \stackrel{\bar{P}}{P}_{\bar{\alpha}}\left(\bar{y}^{0}\right)-\delta_{\beta \bar{\alpha}}\right| \leq C \bar{a} \quad \text { for } \bar{\alpha} \in \overline{\mathcal{A}}, \beta \in \mathcal{M} .
$$

Given $\bar{S} \subset \bar{E}$ with $\#(\bar{S}) \leq k^{\#}$, we let $\tilde{\varphi}_{\bar{\alpha}}^{\bar{S}}$ be as in (36) for each $\bar{\alpha} \in \overline{\mathcal{A}}$. We then define

$$
\overline{\bar{\varphi}} \overline{\bar{\alpha}}=\sum_{\alpha^{\prime} \in \overline{\mathcal{A}}} \tilde{\varphi}_{\alpha^{\prime}}^{\bar{S}} M_{\alpha^{\prime} \bar{\alpha}} \quad \text { for all } \bar{\alpha} \in \overline{\mathcal{A}}
$$

From (36)(a), (37), (2), and (41), we have, for each $\bar{\alpha} \in \overline{\mathcal{A}}$, that

$$
\left|\partial^{\beta} \overline{\bar{\varphi}}_{\bar{\alpha}}^{\bar{S}}\left(\bar{x}^{\prime}\right)-\partial^{\beta} \overline{\bar{\varphi}}_{\bar{\alpha}}^{\bar{S}}\left(\bar{x}^{\prime \prime}\right)\right| \leq\left[C^{\prime}(\bar{a}) \cdot \bar{a}_{0}+C^{\prime \prime} \cdot \bar{a}\right] \cdot \omega\left(\left|\bar{x}^{\prime}-\bar{x}^{\prime \prime}\right|\right)
$$

for $|\beta|=m, \bar{x}^{\prime}, \bar{x}^{\prime \prime} \in \mathbb{R}^{n},\left|\bar{x}^{\prime}-\bar{x}^{\prime \prime}\right| \leq 1$.

Similarly, from (36)(b), (37), (2), and (41), we have, for each $\bar{\alpha} \in \overline{\mathcal{A}}$, that

$$
J_{\bar{x}}(\overline{\bar{\varphi}} \overline{\bar{\alpha}}) \in C^{\prime}(\bar{a}) A^{-1} \bar{\sigma}(\bar{x}) \quad \text { for all } \bar{x} \in \bar{S} .
$$

Also, comparing (38) with (41), and recalling (36)(c), we have, for each $\bar{\alpha} \in \overline{\mathcal{A}}$, that

$$
J_{\bar{y}^{0}}(\overline{\bar{\varphi}} \overline{\bar{\alpha}})=\overline{\bar{P}}_{\bar{\alpha}}
$$

Since (42), (43), (44) hold for the $\overline{\bar{\varphi}} \frac{\bar{S}}{\bar{\alpha}}$, we have proven the following.

(45) Given $\bar{\alpha} \in \overline{\mathcal{A}}$ and $\bar{S} \subset \bar{E}$ with $\#(\bar{S}) \leq k^{\#}$, there exists $\overline{\bar{\varphi}}_{\bar{\alpha}}^{\bar{S}} \in C_{\ell o c}^{m, \omega}\left(\mathbb{R}^{n}\right)$, with

(a) $\left|\partial^{\beta} \overline{\bar{\varphi}} \overline{\bar{\alpha}} \overline{\bar{\alpha}}\left(\bar{x}^{\prime}\right)-\partial^{\beta} \overline{\bar{\varphi}} \overline{\bar{\alpha}}\left(\bar{x}^{\prime \prime}\right)\right| \leq\left[C^{\prime}(\bar{a}) \cdot \bar{a}_{0}+C^{\prime \prime} \bar{a}\right] \cdot \omega\left(\left|\bar{x}^{\prime}-\bar{x}^{\prime \prime}\right|\right)$ for $\bar{x}^{\prime}, \bar{x}^{\prime \prime} \in \mathbb{R}^{n},\left|\bar{x}^{\prime}-\bar{x}^{\prime \prime}\right| \leq 1,|\beta|=m$;

(b) $J_{\bar{x}}\left(\overline{\bar{\varphi}} \frac{\bar{S}}{\bar{\alpha}}\right) \in\left[C^{\prime}(\bar{a}) \cdot A^{-1}\right] \bar{\sigma}(\bar{x})$ for all $\bar{x} \in \bar{S}$; and

(c) $J_{\bar{y}^{0}}(\overline{\bar{\varphi}} \overline{\bar{\alpha}})=\overline{\bar{P}}_{\bar{\alpha}}$.

Next, we establish the Whitney $\omega$-convexity of $\bar{\sigma}(\bar{x})$, and estimate its Whitney constant.

We check that the hypotheses of Lemma 17.1 are satisfied by the sets $\sigma(\tau(\bar{x})), \bar{\sigma}(\bar{x})$, with $c_{0}$ and $C_{0}$ in Lemma 17.1 taken here to be $c(\bar{a})$ and $C$, respectively. In fact, the hypothesis $c_{0}<\lambda_{i} \leq 1$ in Lemma 17.1 holds here, thanks to (12). The hypothesis " $\sigma(\tau(\bar{x}))$ is Whitney $\omega$-convex, with Whitney constant $C_{0}$ " in Lemma 17.1 holds here, thanks to (SL0). We note 
that $\bar{\sigma}(\bar{x})$ is defined from $\sigma(\tau(\bar{x}))$ as in Lemma 17.1, thanks to (8). Finally, the hypothesis " $A$ exceeds a large enough constant determined by $c_{0}, m, n$ " in Lemma 17.1 holds here, thanks to our assumption (3).

Thus, all the hypotheses of Lemma 17.1 hold here, as claimed. Applying that lemma, we now see that the following holds.

(46) For any $\bar{x} \in \bar{E}$, the set $\bar{\sigma}(\bar{x}) \subset \mathcal{R}_{\bar{x}}$ is Whitney $\omega$-convex at $\bar{x}$, with Whitney constant $C$.

In view of (29) (45), (46), (40), and (39), we can pick a controlled constant $C_{1}$ for which the following hold:

(47) For each $\bar{x} \in \bar{E}$, the set $\bar{\sigma}(\bar{x})$ is Whitney $\omega$-convex at $\bar{x}$, with Whitney constant $C_{1}$.

(48) $\partial^{\beta} \stackrel{\bar{P}}{P}_{\bar{\alpha}}\left(\bar{y}^{0}\right)=\delta_{\beta \bar{\alpha}}$ for $\beta, \bar{\alpha} \in \overline{\mathcal{A}}$.

(49) $\left|\partial^{\beta} \overline{\bar{P}}_{\bar{\alpha}}\left(\bar{y}^{0}\right)-\delta_{\beta \bar{\alpha}}\right| \leq C \bar{a}$ for all $\bar{\alpha} \in \overline{\mathcal{A}}, \beta \in \mathcal{M}$.

(50) Given $\bar{\alpha} \in \overline{\mathcal{A}}$ and $\bar{S} \subset \bar{E}$ with $\#(\bar{S}) \leq k^{\#}$, there exists $\overline{\bar{\varphi}}_{\bar{\alpha}}^{\bar{S}} \in C_{\ell o c}^{m, \omega}\left(\mathbb{R}^{n}\right)$, with

(a) $\left|\partial^{\beta} \overline{\bar{\varphi}} \overline{\bar{\alpha}}\left(\bar{x}^{\prime}\right)-\partial^{\beta} \overline{\bar{\varphi}} \overline{\bar{\alpha}}\left(\bar{x}^{\prime \prime}\right)\right| \leq\left[C^{\prime}(\bar{a}) \cdot \bar{a}_{0}+C^{\prime \prime} \cdot \bar{a}\right] \cdot \omega\left(\left|\bar{x}^{\prime}-\bar{x}^{\prime \prime}\right|\right)$ for $|\beta|=m, \bar{x}^{\prime}, \bar{x}^{\prime \prime} \in \mathbb{R}^{n},\left|\bar{x}^{\prime}-\bar{x}^{\prime \prime}\right| \leq 1$;

(b) $J_{\bar{x}}(\overline{\bar{\varphi}} \overline{\bar{\alpha}}) \in\left[C^{\prime}(\bar{a}) \cdot A^{-1}\right] \bar{\sigma}(\bar{x})$ for all $\bar{x} \in \bar{S}$; and

(c) $J_{\bar{y}^{0}}(\overline{\bar{\varphi}} \overline{\bar{\alpha}})=\overline{\bar{P}}_{\bar{\alpha}}$.

(51) Given $\bar{S} \subset \bar{E}$ with $\#(\bar{S}) \leq k^{\#}$, there exists $\bar{F}^{\bar{S}} \in C^{m, \omega}\left(\mathbb{R}^{n}\right)$, with

(a) $\left\|\bar{F}^{\bar{S}}\right\|_{C^{m, \omega}\left(\mathbb{R}^{n}\right)} \leq C_{1}$; and

(b) $J_{\bar{x}}\left(\bar{F}^{\bar{S}}\right) \in \bar{f}(\bar{x})+C A^{-1} \bar{\sigma}(\bar{x})$ for all $\bar{x} \in \bar{S}$.

We prepare to invoke the Weak Main Lemma for $\overline{\mathcal{A}}$. (Recall $\overline{\mathcal{A}} \leq \mathcal{A}$; $($ see $(32))$.)

We now pick a small constant $a_{0}$, for use in the hypotheses of the Weak Main Lemma for $\overline{\mathcal{A}}$. In fact, we take $a_{0}$ to be a controlled constant, small enough to satisfy (WL5) in the Weak Main Lemma for any $\mathcal{A}^{\prime} \leq \mathcal{A}$, with $C$ in (WL0, . ,5) taken here to be $C_{1}$ as in (47) and (51). We can achieve this with a small enough controlled constant $a_{0}$, because $C_{1}$ is a controlled constant. 
We now check that the hypotheses of the Weak Main Lemma for $\overline{\mathcal{A}}$ are satisfied by the following data:

- The constants $C_{1}$ (as in (47) and (51)) and $a_{0}$ (as just discussed).

- The regular modulus of continuity $\omega$.

- The finite set $\bar{E} \subset \mathbb{R}^{n}$.

- The point $\bar{y}^{0} \in \mathbb{R}^{n}$.

- The family of polynomials $\overline{\bar{P}}_{\bar{\alpha}} \in \mathcal{P}$, indexed by $\bar{\alpha} \in \overline{\mathcal{A}}$.

- The $m$-jet $\bar{f}(\bar{x})$ associated to each $\bar{x} \in \bar{E}$.

- The subset $\bar{\sigma}(\bar{x}) \subset \mathcal{R}_{\bar{x}}$ associated to each $\bar{x} \in \bar{E}$.

In fact, for these data, hypothesis (WL5) holds, thanks to our choice of $a_{0}$.

Comparing hypotheses (WL0, . ,4) with our results $(47), \ldots,(51)$ we see the following.

(WL0) for our data is precisely (47).

(WL1) for our data is precisely (48).

(WL2) for our data follows from (49), provided we have

$$
C \bar{a} \leq a_{0} .
$$

(WL3) for our data follows from (50), provided we have

$$
\left[C^{\prime}(\bar{a}) \cdot \bar{a}_{0}+C^{\prime \prime} \bar{a}\right] \leq a_{0}
$$

and

$$
\left[C^{\prime}(\bar{a}) \cdot A^{-1}\right] \leq C_{1} .
$$

(WL4) for our data follows from (51), provided we have

$$
\left[C A^{-1}\right] \leq C_{1}
$$

Hence, to check the hypotheses of the Weak Main Lemma for $\overline{\mathcal{A}}$ for our data, it is enough to check that conditions (52), . ., (55) hold. However, (52) holds, thanks to $(2)$, since we picked $a_{0}$ to be a controlled constant. (In fact, $C$ and $a_{0}$ in (52) are both determined by $C, m, n$ in $(\mathrm{SLO}, \ldots, 5)$; see the definition of "controlled constants".)

Similarly, to check (53), we note that $C^{\prime \prime} \bar{a} \leq \frac{1}{2} a_{0}$, thanks to (2); and $C^{\prime}(\bar{a}) \cdot \bar{a}_{0} \leq \frac{1}{2} a_{0}$, thanks to (4). (Here again, we use the fact that $a_{0}$ is a controlled constant.) Hence, (53) holds.

Finally, (54) and (55) hold, thanks to (3).

This completes the verification of the hypotheses of the Weak Main Lemma for $\overline{\mathcal{A}}$, for the above data. 
We recall that we are assuming that the Weak Main Lemma holds for $\overline{\mathcal{A}}$, since $\overline{\mathcal{A}} \leq \mathcal{A}$. Applying that lemma to our data, we learn the following.

There exists $\bar{F} \in C^{m, \omega}\left(\mathbb{R}^{n}\right)$, with

$$
\begin{aligned}
& \|\bar{F}\|_{C^{m, \omega}\left(\mathbb{R}^{n}\right)} \leq C^{\prime}, \quad \text { and } \\
& J_{\bar{x}}(\bar{F}) \in \bar{f}(\bar{x})+C^{\prime} \bar{\sigma}(\bar{x}) \text { for all } \bar{x} \in \bar{E} \cap B\left(\bar{y}^{0}, c^{\prime}\right) .
\end{aligned}
$$

We fix $\bar{F}$ as above, and define

$$
F=\bar{F} \circ \tau^{-1}
$$

Thus, $F \in C^{m, \omega}\left(\mathbb{R}^{n}\right)$. We estimate its norm. By definition of $\tau$, and by (12), we have from (56) that

$$
\left\|\partial^{\beta} F\right\|_{C^{0}\left(\mathbb{R}^{n}\right)}=\lambda_{1}^{-\beta_{1}} \cdots \lambda_{n}^{-\beta_{n}}\left\|\partial^{\beta} \bar{F}\right\|_{C^{0}\left(\mathbb{R}^{n}\right)} \leq C(\bar{a}),
$$

for $|\beta| \leq m, \beta=\left(\beta_{1}, \ldots, \beta_{n}\right)$.

Also, for $|\beta|=m, \beta=\left(\beta_{1}, \ldots, \beta_{n}\right), x^{\prime}, x^{\prime \prime} \in \mathbb{R}^{n},\left|\tau^{-1}\left(x^{\prime}\right)-\tau^{-1}\left(x^{\prime \prime}\right)\right| \leq 1$, we have

$$
\begin{aligned}
\left|\partial^{\beta} F\left(x^{\prime}\right)-\partial^{\beta} F\left(x^{\prime \prime}\right)\right| & =\lambda_{1}^{-\beta_{1}} \cdots \lambda_{n}^{-\beta_{n}}\left|\partial^{\beta} \bar{F}\left(\tau^{-1}\left(x^{\prime}\right)\right)-\partial^{\beta} \bar{F}\left(\tau^{-1}\left(x^{\prime \prime}\right)\right)\right| \\
& \leq C(\bar{a}) \cdot \omega\left(\left|\tau^{-1}\left(x^{\prime}\right)-\tau^{-1}\left(x^{\prime \prime}\right)\right|\right)
\end{aligned}
$$

Recall that $\omega$ is a regular modulus of continuity, and note that $\mid \tau^{-1}\left(x^{\prime}\right)-$ $\tau^{-1}\left(x^{\prime \prime}\right)|\leq C(\bar{a}) \cdot| x^{\prime}-x^{\prime \prime} \mid$, by (12). Consequently, for a suitable constant $c_{1}(\bar{a})$, we find that $\left|x^{\prime}-x^{\prime \prime}\right| \leq c_{1}(\bar{a})$ implies $\left|\tau^{-1}\left(x^{\prime}\right)-\tau^{-1}\left(x^{\prime \prime}\right)\right| \leq 1$ and $\omega\left(\left|\tau^{-1}\left(x^{\prime}\right)-\tau^{-1}\left(x^{\prime \prime}\right)\right|\right) \leq C(\bar{a}) \cdot \omega\left(\left|x^{\prime}-x^{\prime \prime}\right|\right)$.

Together with (60), this yields

$$
\left|\partial^{\beta} F\left(x^{\prime}\right)-\partial^{\beta} F\left(x^{\prime \prime}\right)\right| \leq C(\bar{a}) \cdot \omega\left(\left|x^{\prime}-x^{\prime \prime}\right|\right)
$$

for $|\beta|=m,\left|x^{\prime}-x^{\prime \prime}\right| \leq c_{1}(\bar{a})$.

On the other hand, if $|\beta|=m, c_{1}(\bar{a}) \leq\left|x^{\prime}-x^{\prime \prime}\right| \leq 1$, then we have $\omega\left(\left|x^{\prime}-x^{\prime \prime}\right|\right) \geq c_{1}(\bar{a})$ since $\omega$ is a regular modulus of continuity, and

$$
\left|\partial^{\beta} F\left(x^{\prime}\right)-\partial^{\beta} F\left(x^{\prime \prime}\right)\right| \leq\left|\partial^{\beta} F\left(x^{\prime}\right)\right|+\left|\partial^{\beta} F\left(x^{\prime \prime}\right)\right| \leq C(\bar{a}) .
$$

Hence,

$$
\left|\partial^{\beta} F\left(x^{\prime}\right)-\partial^{\beta} F\left(x^{\prime \prime}\right)\right| \leq C(\bar{a}) \cdot \omega\left(\left|x^{\prime}-x^{\prime \prime}\right|\right)
$$

if $c_{1}(\bar{a}) \leq\left|x^{\prime}-x^{\prime \prime}\right| \leq 1,|\beta|=m$. 
From (61) and (62), we have

$$
\left|\partial^{\beta} F\left(x^{\prime}\right)-\partial^{\beta} F\left(x^{\prime \prime}\right)\right| \leq C(\bar{a}) \cdot \omega\left(\left|x^{\prime}-x^{\prime \prime}\right|\right)
$$

for $|\beta|=m, x^{\prime}, x^{\prime \prime} \in \mathbb{R}^{n},\left|x^{\prime}-x^{\prime \prime}\right| \leq 1$.

Together with (59), this shows that

$$
\|F\|_{C^{m, \omega}\left(\mathbb{R}^{n}\right)} \leq C(\bar{a}) .
$$

Next, suppose

$$
x \in E \cap B\left(y^{0}, c_{2}(\bar{a})\right),
$$

and set

$$
\bar{x}=\tau^{-1}(x) .
$$

If we take $c_{2}(\bar{a})$ small enough in (64), then we will have

$$
\bar{x} \in \bar{E} \cap B\left(\bar{y}^{0}, c^{\prime}\right), \quad \text { with } c^{\prime} \text { as in (57); }
$$

this follows from (6) and (12), and from the definition of $\tau$.

From (57) and (66) we obtain

$$
J_{\bar{x}}(\bar{F}) \in \bar{f}(\bar{x})+C^{\prime} \bar{\sigma}(\bar{x}) .
$$

Composing with $\tau^{-1}$, we obtain

$$
J_{x}\left(\bar{F} \circ \tau^{-1}\right) \in(\bar{f}(\bar{x})) \circ \tau^{-1}+\left\{C^{\prime} \bar{P} \circ \tau^{-1}: \bar{P} \in \bar{\sigma}(\bar{x})\right\}
$$

From (7), we obtain

$$
(\bar{f}(\bar{x})) \circ \tau^{-1}=[(f(\tau(\bar{x}))) \circ \tau] \circ \tau^{-1}=f(\tau(\bar{x}))=f(x) \quad(\text { see }(65)) .
$$

From (8), we have

$$
\begin{aligned}
\left\{C^{\prime} \bar{P} \circ \tau^{-1}: \bar{P}\right. & \in \bar{\sigma}(\bar{x})\}=\left\{C^{\prime}[A P \circ \tau] \circ \tau^{-1}: P \in \sigma(\tau(\bar{x}))\right\} \\
& =\left\{C^{\prime} A P: P \in \sigma(\tau(\bar{x}))\right\}=C^{\prime} A \sigma(\tau(\bar{x}))=C^{\prime} A \sigma(x)
\end{aligned}
$$

(see (65) again).

Substituting (58), (68), (69) into (67), we learn that

$$
J_{x}(F) \in f(x)+C^{\prime} A \sigma(x) \text { for all } x \in E \cap B\left(y^{0}, c_{2}(\bar{a})\right) .
$$

Our results (63) and (70) look a lot like the conclusions of the Strong Main Lemma for $\mathcal{A}$. However, the constants in (63), (70) depend on $\bar{a}$ and $A$, which do not appear in the Strong Main Lemma. Also, we recall that we are assuming conditions (2), (3), (4) on the additional constants $\bar{a}$ and $A$. We now remove the assumptions (2), (3), (4), and complete the proof of the Strong Main Lemma for $\mathcal{A}$. 
We take $\bar{a}$ to be a controlled constant (ie., determined by $C, m, n$ in $(\mathrm{SL0}, \ldots, 5))$, small enough to satisfy (2). Next, since $\bar{a}$ is controlled, we may take $A$ to be a controlled constant, large enough to satisfy (3). Finally, since $A$ and $\bar{a}$ are controlled, (4) says merely that $\bar{a}_{0}$ is less than a small enough constant determined by $C, m, n$ in (SL0), ..,(SL5). Consequently, (4) follows at once from hypothesis (SL5). Thus, we have taken $A$ and $\bar{a}$ to be controlled constants, for which assumptions (2), (3), (4) are satisfied.

With our $A$ and $\bar{a}$, results (63) and (70) are valid, since assumptions (2), (3), (4) hold. Moreover, since $A$ and $\bar{a}$ are controlled, the quantities $C(\bar{a})$, $C^{\prime} A$, and $c_{2}(\bar{a})$ are controlled constants. Therefore, (63) and (70) show that $F \in C^{m, \omega}\left(\mathbb{R}^{n}\right)$ satisfies

(71) $\|F\|_{C^{m, \omega}\left(\mathbb{R}^{n}\right)} \leq C^{\prime}$, and $J_{x}(F) \in f(x)+C^{\prime} \sigma(x)$ for all $x \in E \cap B\left(y^{0}, c^{\prime}\right)$, with $C^{\prime}$ and $c^{\prime}$ determined by $C, m, n$ in $(\mathrm{SL} 0, \ldots, 5)$.

However, (71) is precisely the conclusion of the Strong Main Lemma for $\mathcal{A}$. Thus, assuming the Weak Main Lemma for all $\overline{\mathcal{A}} \leq \mathcal{A}$, we have proven the Strong Main Lemma for $\mathcal{A}$. The proof of Lemma 5.3 is complete.

\section{Proof of the Main Result}

In this section, we prove Theorem 2 from the Introduction.

We have proven Lemmas 5.1, 5.2, 5.3. Consequently, we have proven the Local Theorem stated in Section 5 ("Plan of the Proof"). That result applies to finite sets $E$. We now remove the finiteness assumption on $E$, by Ascoli's theorem.

Lemma 19.1 There exists $k^{\#}$, depending only on $m$ and $n$, for which the following holds.

Suppose we are given a regular modulus of continuity $\omega$; an arbitrary set $E \subset \mathbb{R}^{n}$; and, for each $x \in E$ an $m$-jet $f(x) \in \mathcal{R}_{x}$ and a subset $\sigma(x) \subset \mathcal{R}_{x}$.

Assume that the following conditions are satisfied.

(1) For each $x \in E$, the set $\sigma(x)$ is Whitney $\omega$-convex at $x$, with Whitney constant $C$.

(2) Given $S \subset E$ with $\#(S) \leq k^{\#}$, there exists $F^{S} \in C^{m, \omega}\left(\mathbb{R}^{n}\right)$, with

$$
\left\|F^{S}\right\|_{C^{m, \omega}\left(\mathbb{R}^{n}\right)} \leq C \text {, and } J_{x}\left(F^{S}\right) \in f(x)+C \sigma(x) \text { for each } x \in S .
$$

Then, for any $y^{0} \in \mathbb{R}^{n}$, there exists $F \in C^{m, \omega}\left(\mathbb{R}^{n}\right)$, with

$\|F\|_{C^{m, \omega}\left(\mathbb{R}^{n}\right)} \leq C^{\prime}$, and $J_{x}(F) \in f(x)+C^{\prime} \sigma(x)$ for all $x \in E \cap B\left(y^{0}, c^{\prime}\right)$.

Here, $C^{\prime}$ and $c^{\prime}$ depend only on $C, m, n$ in (1) and (2). 
Proof: Let $k^{\#}, C^{\prime}, c^{\prime}$ be as in the Local Theorem, suppose $\omega, E, f, \sigma$ satisfy (1) and (2), and let $y^{0} \in \mathbb{R}^{n}$.

Applying the Local Theorem, we learn the following.

(3) Given a finite subset $E_{1} \subset E$, there exists $F \in C^{m, \omega}\left(\mathbb{R}^{n}\right)$, with $\|F\|_{C^{m, \omega}\left(\mathbb{R}^{n}\right)} \leq C^{\prime}$, and $J_{x}(F) \in f(x)+C^{\prime} \sigma(x)$ for all $x \in E_{1} \cap B\left(y^{0}, c^{\prime}\right)$.

Now set

$$
X=\left\{F \in C^{m, \omega}(B):\|F\|_{C^{m, \omega}(B)} \leq C^{\prime}\right\},
$$

where $B$ denotes the closed ball with center $y^{0}$ and radius $c^{\prime}$, and

$$
\|F\|_{C^{m, \omega}(B)}=\max \left\{\max _{\substack{|\beta| \leq m \\ x \in B}}\left|\partial^{\beta} F(x)\right|, \max _{|\beta|=m} \sup _{\substack{x^{\prime}, x^{\prime \prime} \in B \\ 0<\left|x^{\prime}-x^{\prime \prime}\right| \leq 1}} \frac{\left|\partial^{\beta} F\left(x^{\prime}\right)-\partial^{\beta} F\left(x^{\prime \prime}\right)\right|}{\omega\left(\left|x^{\prime}-x^{\prime \prime}\right|\right)}\right\} .
$$

We equip $X$ with the $C^{m}$-topology. Thus, $X$ is compact, by Ascoli's theorem.

For each $x \in E \cap B$, we define

$$
\mathcal{F}_{x}=\left\{F \in X: J_{x}(F) \in f(x)+C^{\prime} \sigma(x)\right\} .
$$

Each $\mathcal{F}_{x}$ is a closed subset of $X$, since the set $\sigma(x)$ is closed. (Recall that, by definition, a Whitney $\omega$-convex set is closed.)

From (3), we see that any finite list $\mathcal{F}_{x_{1}}, \ldots, \mathcal{F}_{x_{N}}\left(x_{i} \in E \cap B\right)$ has nonempty intersection. Since $X$ is compact, it follows that the intersection of all the $\mathcal{F}_{x}(x \in E \cap B)$ is non-empty.

Letting $\tilde{F}$ belong to this intersection, we see that

(4) $\tilde{F} \in C^{m, \omega}(B),\|\tilde{F}\|_{C^{m, \omega}(B)} \leq C^{\prime}$, and $J_{x}(\tilde{F}) \in f(x)+C^{\prime} \sigma(x)$ for all $x \in E \cap B$.

Unfortunately, $\tilde{F}$ is defined only on $B$. To remedy this, we introduce a cutoff function $\theta$ on $\mathbb{R}^{n}$, satisfying

$\theta=1 \quad$ on $B\left(y^{0}, c^{\prime} / 2\right), \quad \operatorname{supp} \theta \subset B\left(y^{0}, c^{\prime}\right) \quad$ and $\quad\|\theta\|_{C^{m, \omega}\left(\mathbb{R}^{n}\right)} \leq C^{\prime \prime}$

determined by $m$ and $n$ and the Whitney constant.

We then define $F=\theta \cdot \tilde{F}$ on $\mathbb{R}^{n}$. From (4) and the defining properties of $\theta$, we deduce easily that $F \in C^{m, \omega}\left(\mathbb{R}^{n}\right)$,

(5) $\|F\|_{C^{m, \omega}\left(\mathbb{R}^{n}\right)} \leq C^{\prime \prime \prime}$ and $J_{x}(F) \in f(x)+C^{\prime \prime \prime} \sigma(x)$ for all $x \in E \cap B\left(y^{0}, c^{\prime} / 2\right)$.

Here, $C^{\prime \prime \prime}$ depends only on $C, m, n$ in (1) and (2). Our result (5) is the conclusion of Lemma 19.1. The proof of the lemma is complete. 


\section{Proof of Theorem 2:}

Let $k^{\#}$ be as in Lemma 19.1, and let $\omega, E, A, f, \sigma$ be as in the hypotheses of Theorem 2. We write $a, A^{\prime}, A^{\prime \prime}$, etc. to denote constants determined by $A, m, n$. Lemma 19.1 tells us that, for suitable constants $A^{\prime}, a^{\prime}$, the following holds.

Given $y \in \mathbb{R}^{n}$, there exists $F^{y} \in C^{m, \omega}\left(\mathbb{R}^{n}\right)$, with

$$
\begin{aligned}
& \left\|F^{y}\right\|_{C^{m, \omega}\left(\mathbb{R}^{n}\right)} \leq A^{\prime}, \quad \text { and } \\
& J_{x}\left(F^{y}\right) \in f(x)+A^{\prime} \sigma(x) \text { for all } x \in E \cap B\left(y, a^{\prime}\right) .
\end{aligned}
$$

To exploit this, we introduce a partition of unity,

$$
1=\sum_{\nu} \theta_{\nu} \quad \text { on } \mathbb{R}^{n}
$$

with

$$
\begin{gathered}
\operatorname{supp} \theta_{\nu} \subset B\left(y_{\nu}, \frac{1}{3} a^{\prime}\right), \\
\left\|\theta_{\nu}\right\|_{C^{m+1}\left(\mathbb{R}^{n}\right)} \leq A^{\prime \prime},
\end{gathered}
$$

We may suppose also that

(11) Any ball of radius 1 in $\mathbb{R}^{n}$ intersects at most $A^{\prime \prime \prime}$ of the balls $B\left(y_{\nu}, a^{\prime}\right)$.

We then define

$$
F=\sum_{\nu} \theta_{\nu} F^{y_{\nu}}
$$

with $F^{y_{\nu}}$ as in (6), (7). From (6), (9), (10), we see easily that

$$
\theta_{\nu} F^{y_{\nu}} \in C^{m, \omega}\left(\mathbb{R}^{n}\right), \quad \text { with }\left\|\theta_{\nu} F^{y_{\nu}}\right\|_{C^{m, \omega}\left(\mathbb{R}^{n}\right)} \leq \tilde{A} .
$$

Together with (11), this shows that $F \in C^{m, \omega}\left(\mathbb{R}^{n}\right)$, with

$$
\|F\|_{C^{m, \omega}\left(\mathbb{R}^{n}\right)} \leq A^{\#}
$$

Next, suppose $x \in E$. We fix $\mu$ with $x \in B\left(y_{\mu}, \frac{1}{3} a^{\prime}\right)$. (There must exist $\mu$ with this property, thanks to (8) and (9).)

Suppose we have any $\nu$, for which $x \in B\left(y_{\nu}, \frac{1}{3} a^{\prime}\right)$. Then (7) gives

$$
J_{x}\left(F^{y_{\mu}}\right), J_{x}\left(F^{y_{\nu}}\right) \in f(x)+A^{\prime} \sigma(x) .
$$


Also, (8), (12) imply that

$$
F=\sum_{\nu} \theta_{\nu} F^{y_{\mu}}+\sum_{\nu} \theta_{\nu} \cdot\left(F^{y_{\nu}}-F^{y_{\mu}}\right)=F^{y_{\mu}}+\sum_{\nu} \theta_{\nu} \cdot\left(F^{y_{\nu}}-F^{y_{\mu}}\right)
$$

on $\mathbb{R}^{n}$, hence

$$
J_{x}(F)=J_{x}\left(F^{y_{\mu}}\right)+\sum_{\nu} J_{x}\left(\theta_{\nu}\right) \cdot J_{x}\left(F^{y_{\nu}}-F^{y_{\mu}}\right),
$$

with the multiplication performed in $\mathcal{R}_{x}$.

From (14), we see that

$$
J_{x}\left(F^{y_{\nu}}-F^{y_{\mu}}\right) \in 2 A^{\prime} \sigma(x) \text { if } B\left(y_{\nu}, \frac{1}{3} a^{\prime}\right) \text { contains } x .
$$

From (6), we have that

$$
\left|\partial^{\beta}\left[J_{x}\left(F^{y_{\nu}}-F^{y_{\mu}}\right)\right](x)\right| \leq\left|\partial^{\beta} F^{y_{\nu}}(x)\right|+\left|\partial^{\beta} F^{y_{\mu}}(x)\right| \leq 2 A^{\prime}
$$

for $|\beta| \leq m$.

From (10), we have

$$
\left|\partial^{\beta}\left[J_{x}\left(\theta_{\nu}\right)\right](x)\right| \leq A^{\prime \prime} \quad \text { for }|\beta| \leq m .
$$

In view of the hypothesis of Theorem 2, to the effect that $\sigma(x)$ is Whitney $\omega$-convex with Whitney constant $A$, we learn from $(16),(17),(18)$ that

$$
J_{x}\left(\theta_{\nu}\right) \cdot J_{x}\left(F^{y_{\nu}}-F^{y_{\mu}}\right) \in A^{*} \sigma(x) \text { if } B\left(y_{\nu}, \frac{1}{3} a^{\prime}\right) \text { contains } x .
$$

Also, if $x \notin B\left(y_{\nu}, \frac{1}{3} a^{\prime}\right)$, then $J_{x}\left(\theta_{\nu}\right)=0$, by (9).

Therefore, (14), (15), (19) together imply that

$$
\begin{array}{r}
J_{x}(F)=J_{x}\left(F^{y_{\mu}}\right)+\sum_{B\left(y_{\nu}, a^{\prime} / 3\right)_{\ni} x} J_{x}\left(\theta_{\nu}\right) \cdot J_{x}\left(F^{y_{\nu}}-F^{y_{\mu}}\right) \\
\in\left(f(x)+A^{\prime} \sigma(x)\right)+\sum_{B\left(y_{\nu}, a^{\prime} / 3\right)_{\ni} x} A^{*} \sigma(x) .
\end{array}
$$

This in turn implies

$$
J_{x}(F) \in f(x)+A^{* *} \sigma(x),
$$

thanks to (11). Thus, we have proven that

$$
J_{x}(F) \in f(x)+A^{* *} \sigma(x) \text { for all } x \in E .
$$

We have exhibited a function $F \in C^{m, \omega}\left(\mathbb{R}^{n}\right)$, satisfying (13) and (20). The constants $A^{\#}$ in (13) and $A^{* *}$ in (20) are determined in $A, m, n$ in the hypotheses of Theorem 2 .

Thus, (13) and (20) are the conclusions of Theorem 2.

The proof of the theorem is complete. 


\section{References}

[1] Bierstone, E., Milman, P. and Pawlucki, W: Differentiable functions defined on closed sets. A problem of Whitney. Invent. Math. 151 (2003), no. 2, 329-352.

[2] Bierstone, E., Milman, P. and Pawlucki, W: Higher-order tangents and Fefferman's paper on Whitney's extension problem. Ann. of Math. (2), to appear.

[3] Brudny $\breve{I}$, Y.: On an extension theorem. Funkcional. Anal. i Priložen 4 (1970), no. 3, 97-98. English translation in Funct. Anal. Appl. 4 (1970), $252-253$.

[4] Brudny $\breve{\text {, }}$ Y. And Shvartsman, P.: A linear extension operator for a space of smooth functions defined on closed subsets of $\mathbb{R}^{n}$. Dokl. Akad. Nauk SSSR 280 (1985), no. 2, 268-272.

[5] Brudny $\breve{I}$, Y. and Shvartsman, P.: Generalizations of Whitney's extension theorem. Internat. Math. Res. Notices (1994), no. 3, 129-139.

[6] Brudny̆, Y. and Shvartsman, P.: The Whitney problem of existence of a linear extension operator. J. Geom. Anal. 7 (1997), no. 4, 515-574.

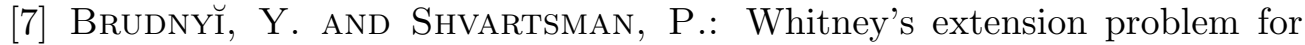
multivariate $C^{1, w}$ functions. Trans. Amer. Math. Soc. 353 (2001), no. 6, $2487-2512$.

[8] Fefferman, C.: Interpolation and extrapolation of smooth functions by linear operators. Rev. Mat. Iberoamericana 21 (2005), no. 1, 313-348.

[9] Fefferman, C.: A sharp form of Whitney's extension theorem. Ann. of Math. (2) 161 (2005), no. 1, 509-575.

[10] Fefferman, C.: Whitney's extension problem for $C^{m}$. Ann. of Math. (2), to appear.

[11] Fefferman, C.: Whitney's extension problem in certain function spaces, preprint.

[12] Glaeser, G.: Études de quelques algèbres tayloriennes. J. Analyse Math. 6 (1958), 1-124.

[13] Malgrange, B.: Ideals of Differentiable Functions. Oxford University Press, Oxford, 1966.

[14] Shvartsman, P.: Shvartsman, P.: Lipschitz selections of multivalued mappings and traces of the Zygmund class of functions to an arbitrary compact. Dokl. Acad. Nauk SSSR 276 (1984), no. 3, 559-562. English translation in Soviet Math. Dokl. 29 (1984), 565-568.

[15] Shvartsman, P.: Traces of functions of Zygmund classes. Sibirsk. Mat. Zh. 28 (1987), no. 5, 203-215. English translation in Siberian Math. J. 28 (1987), 853-863.

[16] Shvartsman, P.: Lipschitz selections of set-valued functions and Helly's theorem. J. Geom. Anal. 12 (2002), no. 2, 289-324. 
[17] Stein, E. M.: Singular Integrals and Differentiability Properties of Functions. Princeton Univ. Press, Princeton, 1970.

[18] Webster, R.: Convexity. Oxford Science Publications. The Clarendon Press, Oxford University Press, New York, 1994.

[19] Whitney, H.: Analytic extensions of differentiable functions defined in closed sets. Trans. Amer. Math. Soc. 36 (1934), no. 1, 63-89.

[20] Whitney, H.: Differentiable functions defined in closed sets I. Trans. Amer. Math. Soc. 36 (1934), no. 2, 369-387.

[21] Whitney, H.: Functions differentiable on the boundaries of regions. Ann. of Math. (2) 35 (1934), no. 3, 482-485.

[22] Zobin, N.: Whitney's problem on extendability of functions and an intrinsic metric. Adv. Math. 133 (1998), no. 1, 96-132.

[23] Zobin, N.: Extension of smooth functions from finitely connected planar domains. J. Geom. Analysis 9 (1999), no. 3, 491-511.

Recibido: 30 de octubre de 2003

\author{
Charles Fefferman \\ Department of Mathematics \\ Princeton University \\ Fine Hall \\ Washington Road \\ Princeton, New Jersey 08544, USA \\ cf@math.princeton.edu
}

Supported by Grant No. DMS-0245242. 